U.S. Department

of Transportation

National Highway

Traffic Safety

Final Report

Administration

\title{
Impact of Travel Patterns and Driving Behavior on Crash Involvement
}

Stephen D. Benson, Ph.D.

V. Lance Tarrance \& Associates

8150 Leesburg Pike, Suite 6000

Vienna, VA 22180

Contract No. DTNH22-83-R-07322

Contract Amount $\$ 55,000$ 
This document is disseminated under the sponsorship of the Department of Transportation in the interest of Information exchange. The United States Government assumes no liability for 1 ts contents or use thereof. 
Technical Report Documentotion Page

\begin{tabular}{|c|c|}
\hline $\begin{array}{l}\text { I. Ravorl No. } \\
\text { DOT HS-806 } 458\end{array}$ & 3. Recipient's Catalog No. \\
\hline \multirow{2}{*}{$\begin{array}{l}\text { 4. Trile and juot:l: } \\
\text { Impact of Travel Patterns and Driving Behavior } \\
\text { on Crash Involvement }\end{array}$} & $\begin{array}{l}\text { 5. Report Date } \\
\text { July 21, } 1983\end{array}$ \\
\hline & 5. Performing Organization Cude \\
\hline $\begin{array}{l}\text { 7. Author'si } \\
\text { Stephen D. Benson, Ph.D. }\end{array}$ & 3. Performing Orgonization Resolit No. \\
\hline \multirow[t]{2}{*}{$\begin{array}{l}\text { 9. Performing Orgenization Nome onj iddress } \\
\text { V. Lance Tarrance \& Associates } \\
8150 \text { Leesburg Pike, Suite } 600 \\
\text { Vienna, Va. } 22180\end{array}$} & 10. Work Unit No. (TRAIS) \\
\hline & \multirow{2}{*}{$\begin{array}{l}\text { 13. Type of Reporf ond Period Covered } \\
\text { Final Report } \\
3 / 83-7 / 83\end{array}$} \\
\hline $\begin{array}{l}\text { 12. Sponsoring Agency Nome und Address } \\
\text { National Highway Traffic Safety Administration } \\
\text { Office of Driver \& Pedestrian Research NRD- } 40\end{array}$ & \\
\hline 4007 th St., S.W. Washington, D.C. 20590 & 14. Sponsoring Agency Code \\
\hline
\end{tabular}

15. Supplementary Notes

16. Abstract

This study was conducted to obtain statistically valid aata on a variety of highway safety issues, such as attitudes toward drunk driving, use of safety telts and chiid safety seats, and to establish data regarifing triying patterns. The stujy design provided a unique opportunity to deterinine what, if any, relations existed between the responients $5^{\circ}$ attitudes toward drunk driving and the use of sccupant protectton devices and how those drtitudes afiected their driving patterns. This study was dene in three phases. Phase I was a series of focus groups tn assist il the development of the survey instriument. Phase iI was 3 rationwide telephnne survey of 1,000 households, as weil as a sample of 200 respondents who had been interviewed by telephone approximately 18 months earlier. Phase III of the study was a final set of focus groups which were used to interpret data from the teienhorie survay and in r.roviding a sense or the qualitative intensity or results.

Some of the major findings and recommendations that resulted from this study are: (1) The two most important highiway safety issues were reducing the number of people drinking and driving and increasing the use of safoty bults. (2) The tiwo reasons most often cited for the recent decline in traffic deaths were "lower speeds" and "less drunk driving". (3) There has been positive movement in the percentage of individuals who can be categorized as confirmed seat belt users, and those who are categarized as infrequent wearers, when compared to the August 1981 survey. (4) About $63 \%$ of the adult population drink alcoholic beverages (at least occasionally). Of this group. about 206 drive within one hour of drinking at least once a month and another $29 \%$ report :hat they dn sn between one and fcur times a year. The remaining $50 \%$ repert that they never drive within one hour of drinking. (5) Of those who drink and drive, about 24\% repurt that their fraquency of drinking and driving has decreased in the past year. (6) About half of the respondents report that they have personally made an effort to reduce their drinking and driving in the (8) About $85 x$ of (7) Yery few. of the respondents could give any valid ways of "telling when you had too much to dink". (8) About $85 x$ of the respondents had heard of Mothers Against Orunk Oriving (MAOO), and the large majority of them were able ts give valid response to "do you know what they are trying to do". Only 9\% of the respondents who knew about MAD0 ielt they were not succeeding, while $55 \%$ felt that they were succeoding. (9) About three times as many people indicate that they are wearing safety belts more of ten rather than less often when cumpared to a year ago. The major reasons for the increased uses ware: io je safer, more aware of crazy drunk drivers, and for the children. The major reasuns fur the reduced use were: uncoinfortable, brake habit, and quit taking long lrips. (10) About 70\% of the respondents agres with the statement: "putting on a seat belt is just like brushing your teeth and watching your diet." (II) People respond posilively to safety belt messages when the theme is based on either preventative heaith or the other guy. (12) The public perceives 3 strong and effective effort to get the druilk oriver off the road and attributes the success of the program to the "various community efforts" and not to the government.

The following are selected recommendations: (1) A message which tells people how the inertial belt functions nust be devel. oped. Confidence in the fact that the balts are functioning correctly is a prerequisite to attending to any other me:sages. (2) Salety belt usage should be positioned ds a part of the overall preventative health regimen that most people practice. This places the onus for using the belt on the "other guy". (3) Given the public's positive reactions to community efforts (e.g. MA00) to reduce drunk driving, these efforts stould be supported. (4) :similar community efforts should be fostered in the safety belt effort. (5) Programs directed at reducing drunk driving should also emphasize the use of the safety beit as a good defense

\begin{tabular}{l|l} 
17. Koy Words & 18. Distribution Statement
\end{tabular}

Highway safety, drunk driving, safety belts, child safety seats, occupant protector devices, auto travel patterns

Document is available to U.S. public through National Technical Information Service, Springfield, Va. 22161
19. Security Clossif. (of this coport)

Unclassified
20. Security Clossif. (of this poge)

Unclassified

\begin{tabular}{l|l}
$\begin{array}{l}\text { 21. No. of Pages } \\
254\end{array}$ & $\begin{array}{r}\text { 22. Price } \\
\$ 55,000\end{array}$
\end{tabular}




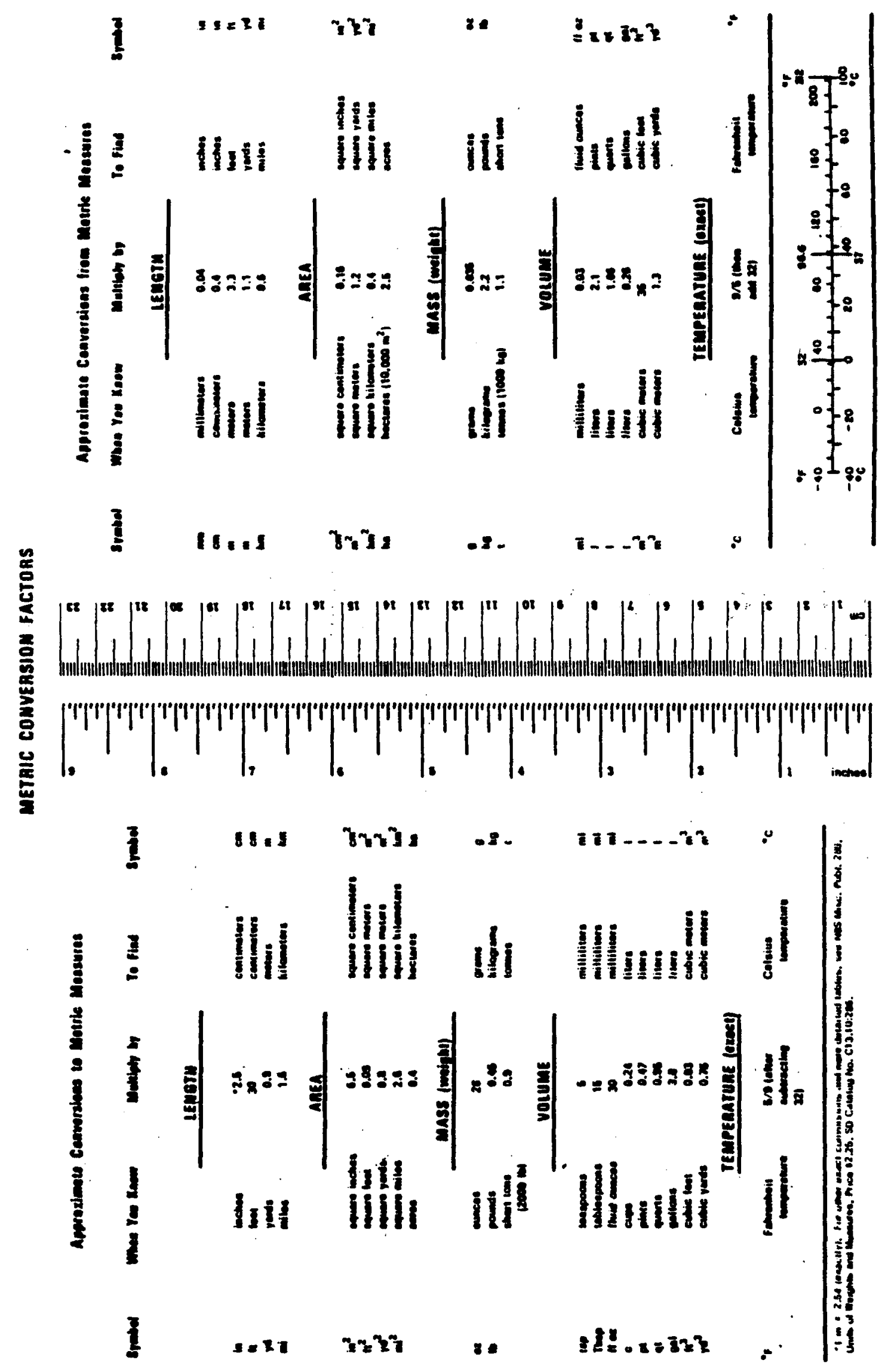


ABLE OF CONTENTS

PAGE

ACKNOWLEDGEMENTS ...................... ix

EXECUTIVE SUMMARY. . . . . . . . . . . . . . . . . . 1

BACKGROUND .......................... 5

SURVEY METHODOLOGY . . . . . . . . . . . . . . . . . . 7

NATIONWIDE SAMPLE BREAKDOWN BY STATE . . . . . . . . . . . . . 9

NATIONWIDE SAMPLE BREAKDOWN BY STANDARD FEDERAL

ADMINISTRATIVE REGIONS ................. . . . 10

CHAPTER I: MAJOR HIGHWAY SAFETY ISSUES. . . . . . . . . . 11

CHAPTER II: SELF-REPORT OF SAFETY BELT USAGE . . . . . . . . 21

CHAPTER III: WHO DRINKS AND DRIVES?............ . 34

CHAPTER IV: DRIVING PATTERNS .............. . . 54

CHAPTER V: PERCEPTION OF RISKS AS AN ISSUE IN THE

USE OF SAFETY BELTS AND DRIVING AND DRINKING . . 94

CHAPTER VI: THE PRODUCT'S IMAGE. . . . . . . . . . . . . 115

CHAPTER VII: MOTHERS AGAINST DRUNK DRIVING. . . . . . . . 146

APPENDIX A

INTERVIEW SCHEDULE IN FIELD ORDER (BRUSHFIRE) 162

APPENDIX B

INTERVIEW SCHEDULE IN FIELD ORDER (PANEL) 201 


\section{TABLE OF CONTENTS}

TABLES

PAGE

Table 1: Verbatims - Reason for Decline in Auto Fatalities 15

Table 2: Verbatims - How Driving Habits Have Changed 17

Table 3: Verbatims - Why Driving Hábits Have Changed 19

Table 4: Verbatims - Why Use Safety Belts More 24

Table 5: Verbatims - Why Use Safety Belts Less 26

Table 6: Self-reported Use by Standard Federal Administrative Regions $\quad 28$

Table 7: Frequency of Driving and Drinking by Drivers/Drinkers and by Total Sample 35

Table 8: Demographics by Frequency Drink and Drive 36

Table 9: Codes - Why Change Drinking and Driving 38

Table 10: Places People Drink Then Drive . 40

Table 11: What Done to Change Drinking and Driving 43

Table 12: Indications - Can't Drink and Drive Safely 44

Table 13: Verbatims - Why Has Drinking/Oriving Changed 52

Table 14: Demographics by Miles Driven Past Year 55

Table 15: Demographics by Miles Driven Weekdays 57

Table 16: Demographics by Miles Driven Weekends 59

Table 17: Number of Trips Over 200 Miles/Past Year 61

Table 18: Change in Number of Trips Over 200 Miles 63

Table 19: Year of Last Over 200-Mile. Trip 65

Table 20: Season of Last 200 Mile Trip 67

Table 21: Number of Persons in Car/Last Trip 69

Table 22: Drove More or Less Than 1982

Table 23: Changed Driving Habits in Past Year 73 
TABLE OF CONTENTS (TABLES) CONTINUED

PAGE

Table 24: Drove More/Less This Holiday Season 75

Table 25: Codes - Why Use Car More This Holiday 77

Table 26: Codes - Why Use Car Less This Holiday 79

Tab7e 27A: Verbatims - Why Drove More in 1982

Table 27B: Verbatims - Why Drove Less in 1982

Table 28A: Verbatims - Why Drove More This Holiday 90

Table 28B: Verbatims - Why Drove Less This Holiday 92

Table 29: Demographics by Wear Belts When Conditions Dangerous 95

Table 30: Demographics by Good Driver/Unsure Other Drivers 98

Table 31: Risk of Punishment for Driving and Drinking 100

Table 32: Demographics by No Accidents/In Control 102

Table 33: Demographics by Effect of Belt on Control of Car 104

Table 34: Demographics by In Control/Can Prevent Accidents 106

Table 35: Demographics by Perceived Cause of Accident 108

Table 36: Passenger Car Fatalities by Seating Position. 111

Table 37: Demographics by Belts Are Like Toothbrush 116

Table 38: Demographics by Degree of Inconvenience _... 118

Table 39: Demographics by Smoking Habits 120

Table 40: Demographics by Frequency Visit Dentist 122

Table 41: Demographics by Exercise Habits 124

Table 42: Demographics by Health Activity 126

Table 43: Demographics by Seat Belts Too Loose/Don't Work 129

Table 44: Demographics by No Accidents/Drive Defensively 132

Table 45: Demographics by Belt Use vs. Insurance 134

Table 46: Demographics by Belt Use: Safety vs. Imposition 136 
TABLE OF CONTENTS (TABLES) CONTINUED

PAGE

Table 47: Demographics by Buzzer: Reminder vs. Ignore 138

Table 48: Demographics by Use Belt: Less/More Serious Injuries 140

Table 49: Demographics by Aware of MADD 148

Table 50: Codes - What is MADD 150

Table 51: Demographics by Is MADD Succeeding 152

Table 52: Codes - Why You Think MADD is Succeeding 154

Table 53: Verbatims - What is MADD 158

Table 54: Verbatims - Why You Think MADD is Succeeding 160 


\section{TABLE OF CONTENTS}

\section{FIGURES}

PAGE

Figure 1: Most Important - Making Driving Safer 12

Figure 2: Major Cause for Decline in Traffic Deaths 14

Figure 3: Self-reported Use 22

Figure 4: Self-reported Use by Change in Use 23

Figure 5: Self-reported Use by Age $\quad 31$

Figure 6: Self-reported Use by Education 32

Figure 7: Age by Change in Use. 33

Figure 8: Frequency Drink/Drive by Reduce Drink/Drive by Age 45

Figure 9: Frequency Drink/Drive by Education 46

Figure 10: Frequency Drink/Drive by Age 47

Figure 11: Frequency Drink/Drive by Stop Stated Time by Age 48

Figure 12: Frequency Drink/Drive by No Alcohol by Age 49

Figure 13: Frequency Drink/Drive by Have Other Drive by Age 50

Figure 14: Frequency Drink/Drive by Limit Drinks by Age 51

Figure 15: Age by Change Driving Habits by Frequency Drink/Drive 82

Figure 16: Age by Car Use Hollidays by Frequency Drink/Drive 83

Figure 17: Age by Car Use vs. Last Year by Frequency Drink/Drive 84

Figure 18: Frequency Drink/Drive by Car Use Holidays by Age 85

Figure 19: Self-reported Use by Belts Affect Control by Frequency Drink/Drive

Figure 20: Self-reported use by No Accidents/In Control by Frequency Drink/Drive

Figure 21: Self-reported Use by Use Belts When Dangerous by Frequency Drink/Drive 
TABLE OF CONTENTS (FIGURES) CONTINUED)

PAGE

Figure 22: Self-reported Use by Belts Loose by Frequency Drink/Drive

Figure 23: Self-reported Use by Belts Safety vs. Imposition by Frequency Drink/Drive

Figure 24: Self-reported Use by Belts vs. Insurance by Frequency Drink/Drive

Figure 25: Self-reported Use by No Accidents/Drive Defensively by Frequency Drink/Drive

Figure 26: Frequency Drink/Drive by Aware MADD by Age 


\section{ACKNOWLEDGEMENTS}

The authors of this report wish to express their sincere gratitude to the Contract Technical Manager, Bruce Bigelow, for his encouragement and technical insights during conduct of this project. Charles F. Rund and Dr. Frank Newport provided invaluable support in the areas of questionnaire design and data analysis. In addition, this study could not have been successfully completed without the assistance of Mrs. Phyllis Maier and Mrs. Imogene Huber of the Houston staff of Tarrance \& Associates who supervised the survey team and handled the multitude of arrangements and logistics necessary for a study of this type. Arlene Durso, Janice Erickson and Shirley Vickery handled the major editorial and typing efforts on the project.

Stephen D. Benson, Ph. D. 


\section{EXECUTIVE SUMMARY}

This study was conducted by Tarrance and Associates as one of its ongoing rational surveys which are designed to obtain data on various national issues, in this case, highway safety. This study was done in three phases. Phase I was a series of focus groups to assist in the development of the survey instrument. Phase II was a nationwide telephone survey of 1,000 households, as well as a sample of 200 respondents who had been interviewed by telephone approximately 18 months earlier. Phase III of the study was a final set of focus groups. These focus groups are helpful in interpreting the data from the telephone survey and in providing a sense of the qualitative intensity of results which the absolute numbers cannot provide. The following is a list of the major findings and recommendations that resulted from this study:

1. The two most important highway safety issues as reported by the respondents were reducing the number of people drinking and driving and increasing the use of safety belts.

2. The two reasons most often cited for the recent decline in traffic deaths were "lower speeds" and "less drunk driving".

3. There has been positive movement in the percentage of individuals who can be categorized as confirmed seat belt users, and those who are categorized as infrequent wearers, when compared to the August 1981 survey.

4. About $63 \%$ of the adult population drink alcoholic beverages (at least occasionally). Of this group, about $20 \%$ drive within one hour of drinking at least once a month and another $29 \%$ report that they do so between one and four times a year.

5. Of those who drink and drive, about $24 \%$ report that their frequency of drinking and driving has decreased in the past year, while $70 \%$ indicate that they are not sure that their frequency of drinking and driving has changed. 
6. About $23 \%$ of those who have reduced their drinking and driving attribute this reduction to stricter DWI laws. Approximately another $35 \%$ respond with answers which can be categorized as an increased awareness of the drunk driving problem.

7. About $48 \%$ of the respondents who drink and drive felt that the chances of getting "caught driving drunk" would have to be at least 1 out of 100 before they would stop drinking and driving. About thirty-five percent (35\%) of those who drink and drive believe that if they drive drunk 100 times they would either never get caught, or caught only once.

8. About $50 \%$ of the drivers/drinkers report that they have personaliy made an effort to reduce their drinking and driving in the last six months. The two most popular ways of doing this were limiting the number of drinks, and having someone else drive home.

9. Very few of the respondents could give any valid ways of "telling when you had too much to drink". The most often reported way was "the numlier of drinks", but only $12 \%$ of the respondents mentioned it. The next most common responses were "wavering/staggering", "general behavior", and "can just tell".

10. About $85 \%$ of the respondents had heard of Mothers Against Drunk Driving (MADD), and the large majority of those who had heard about MADD were able to give a valid response to "do you know what they are trying to do". The most frequent responses were to prevent drunk driving, and to strengthen drunk driving laws.

11. Only $9 \%$ of the respondents who knew about MADD felt they were not succeeding. while $55 \%$ felt that they were succeeding. 
12. About $30 \%$ of the respondents reported a change in driving habits in the last year. The most frequent responses in terms of how they changed focused on increased caution, (e.g. took defensive driving course), use safety belts more, obey speed limits, and stopped drinking and driving.

13. When asked about the amount of driving they did between Thanksgiving and New Years of 1982 as compared to 1981, twice as many respondents indicated that they had "driven less" as indicated they had "driven more".

14. About three times as many people indicate that they ara wearing safety belts more often rather than less often when compared to a year ago.

15. The major reasons for the increased uses were: to be safer, more aware of crazy drunk drivers, and for the children. The major reasons for the reduced use were: uncomfortable, broke habit, and quit taking long trips.

16. Just over half of the respondents don't know how the inertial seat belts function.

17. About $70 \%$ of the respondents agree with the statement putting on a seat belt is just like brushing your teeth and watching your diet.

18. Most people (93\%) feel that they have at least some control in preventing their own involvement in an automobile accident, while only 6\% feel that they would be the most likely cause of an accident. Accidents are caused by the other guy.

19. People respond positively to safety belt messages when the theme is based on either preventive health or the other guy. 
20. The public perceives a strong and effective effort to get the drunk driver off the road and attributes the success of the program to the "various community efforts" and not to the government.

21. Recommendation: A message which tells people how the inertial belt functions must be developed. If people think the belts in their car don't function, then there is no reason to attend to any safety belt message. Confidence in the fact that the belts are functioning correctly is a prerequisite to attending to any other messages.

22. Recommendation: Safety belt usage should be positioned as a part of the overall preventive health regimen that most people practice. This places the onus for using the belt or the "other guy".

23. Recommendation: Given the public's positive reactions to community efforts (e.g., MADD) to reduce drunk driving, these efforts should be supported.

24. Recommendation: Similar community efforts should be fostered in the safety belt effort.

25. Recommendation: Programs. directed at reducing drunk driving should also emphasize the use of the safety belt as a good defense against a drunk driver. 


\section{BACKGROUND}

In calendar year 1982, traffic fatalities fell significantly from earlier trends and predictions. While this reduction is certainly welcome, regardless of cause, it is vitally important that the National Highway Traffic Safety Administration understand, as far as possible, the reasons behind the drop in highway deaths in order to provide whatever guidance it can to its national programs. A number of hypotheses have been proposed to account for the fatality reduction, among them changes in travel patterns, the public's perception of the risks of driving, the increased use of safety belts, and the increased awareness of the drunk driving problem. The objective of this study is to analyze data to determine if there have been significant changes in travel patterns and if significant changes can be attributed to specific motivational or attitudinal factors.

The specific objectives of the study were to determine if:

1) Have there been measurable changes in public travel patterns in 1982 which might account for the reduced deaths on the highways? If so, did these changes manifest themselves in switches from one mode of travel to another? Did they result in fewer trips being taken or in trips taken at different times during the day or week? Can such differences be associated with any identifiable segment of the population? What basic reasons could account for the differences?

2) Have there been identifiable and measurable changes in driving behavior, particularly as it applies to safety issues? That is, have people changed the way they drive because they believe they should drive more safely? Some possible measures of safe driving behaviors could include average speed, following distance, use of safety 
belts under certain conditions, incidence of drinking and driving, or attempts to infiluence other people's driving behavior (e.g., drinking and driving, safety belt use).

3) What basic reasons could account for either of these changes, if differences appear? Is there an association between changes in travel patterns and changes in driving behavior? Can changes in travel patterns in any way be attributable to changed attitudes about safety? Do other reasons, such as economic factors, appear as significant causal factors? Do people who have changed their traveling behavior have different perceptions of the risks of driving than had previously been the case?

4) If differences in perceptions of risk do appear, are they in any way associated with the increased national emphasis on minimizing driving while intoxicated and on the need for using available occupant protective devices (safety belts and child safety belts)? 
SURVEY METHODOLOGY

In order to adequately address the issues of change as required by the Statement of Work Survey, a number of items that have been used in previous surveys done by Tarrance and Associates and others were used in this survey. In addition, a sample of 200 individuals who responded to the 1981 Tarrance survey were recontacted and asked to respond to this survey. Where applicable, the data for this sample of 200 will be presented.

This project presented a unique opportunity to obtain detailed data from the same respondent set in a number of different areas such as attitudinal and self-reported data regarding safety belts, drinking and driving patterns, and travel patterns.

This report contains the results of a telephone survey of 1200 American adults. Responses to this survey were gathered March 25 - April 2, 1983.

All respondents interviewed in this study were part of a fully representative sample of the American adult population. The confidence interval associated with a sample of this type is such that:

- $90 \%$ of the time results will be within $\pm 2.6 \%$ of the "true values";

- $95 \%$ of the time results will be within $\pm 3.1 \%$ of the "true values";

- $99 \%$ of the time results will be within $\pm 4.0 \%$ of the "true values";

where "true values" refer to the results obtained if it were possible to interview every adult in the country. 
Interviewing was conducted by Tarrance \& Associates' instructed personnel working from the company's own telephone bank in Houston, Texas. The interviews lasted approximately 21 minutes. The mean number of calls attempted for each completed interview was 6.5 with a mean refusal rate of 1.5. Randomly generated numbers totaled 238 of the sample universe. Editing, coding and computer processing of the data was done at Tarrance \& Associates' headquarters. The computer tabulations were produced by a private statistical analysis program. 
NATIONWIDE SAMPLE BREAKDOWN BY STATE

$$
(N=1,000)
$$

\begin{tabular}{|c|c|c|c|}
\hline State & Number of Sample & State & Number of Sample \\
\hline Al abama & 17 & New Hampshire & 4 \\
\hline Arizona & 12 & New Jersey & 33 \\
\hline Arkansas & 10 & New Mexico & 6 \\
\hline California & 105 & New York & 78 \\
\hline Colorado & 13 & North Carolina & 26 \\
\hline Connecticut & 14 & North Dakota & 3 \\
\hline Delaware & 3 & Ohio & 48 \\
\hline Florida & 43 & Oklahoma & 14 \\
\hline Georgia & 24 & Oregon & 12 \\
\hline Idaho & 4 & Pennsylvania & 53 \\
\hline Illinois & 51 & Rhode Is land & 4 \\
\hline Indiana & 24 & South Carolina & 14 \\
\hline Iowa & 13 & South Dakota & 3 \\
\hline Kansas & 11 & Tennessee & 20 \\
\hline Kentucky & 16 & Texas & 63 \\
\hline Louisiana & 19 & Utah & 7 \\
\hline Maine & 5 & Vermont & 2 \\
\hline Maryland & 19 & Virginia & 24 \\
\hline Massachusetts & 26 & Washington & 18 \\
\hline Michigan & 41 & West Virginia & 9 \\
\hline Minnesota & 18 & Wisconsin & 21 \\
\hline Mississippi & 11 & Wyoming & 2 \\
\hline Missouri & 22 & & \\
\hline Montana & 4 & & \\
\hline Nebraska & 7 & & \\
\hline Nevada & 4 & & \\
\hline
\end{tabular}


SAMPLE BREAKDOWN BY

STANDARD FEDERAL ADMINISTRATIVE REGIONS

$(\mathrm{N}=1,000)$

Region

I. NEW ENGLAND STATES

(Maine, New Hampshire, Massachusetts,

Connecticut, Vermont, Rhode Island)

II. NEW YORK/NEW JERSEY

(New York, New Jersey)

III. MID-ATLANTIC

(Maryland, Delaware, Pennsylvania,

Virginia, West Virginia)

IV. SOUTHEAST

(A)abama, Florida, Georgia, Kentucky, North Carolina, Tennessee, South

Carolina, Mississippi)

v. GREAT LAKES

(Michigan, Minnesota, Wisconsin,

Illinois, Indiana, Ohio)

VI. MID-SOUTH

(New Mexico, Oklahoma, Louisiana, Texas, Arkansas)

VII. PLAINS

(Nebraska, Kansas, Iowa, Missouri)

VIII. NORTHWEST PLAINS

(Montana, North Dakota, South Dakota, Wyoming, Utah, Colorado)

IX. WEST COAST

(California, Arizona, Nevada)

X. NORTHWEST

(Idaho; Oregon, Washington)
Number of Sample 


\section{MAJOQ HIGHWAY SAFETY ISSUES}

The first substantive question that each respondent was asked in both the 1981 Tarrance \& Associates survey (Tarrance I) and the 1983 Tarrance \& Associates survey (Tarrance II) was: "As you may know, there are many different things we can do to increase auto safety and de:rease the number of injuries and deaths which result from auto accidents. Which of the following do you think would be most important in making driving safer? And which do you think would be the second most important?"

The list that was read was:

1. Less people drinking and driving.

2. More people wearing seat belts.

3. Requiring motorcyclists to wear helmets.

4. Stricter enforcement of traffic laws.

5. Safer cars.

6. Don't know/no answer.

The changes that occurred in the 18 months between Tarrance I and II indicate a demonstrative focusing of the public's perception of the major highway safety issues in 'two areas in the last 18 months. Figure 1 shows the combined averages for both the first and second most important issues for Tarrance I and Tarrance II.

Given that this was the first substantive question asked, it is difficult to assume that the respondents would have developed a strong response set to the issue. The more reasoned interpretation is that the awareness of these two issues is the result of the increased activity at both the national and local levels.. Another question which was asked the respondents in Tarrance II was:

"The total number of auto fatalities and the number of auto fatalities per mile driven dropped significantly last year. What do you think was the major cause for this drop?" 


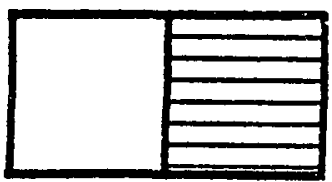

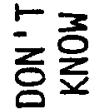

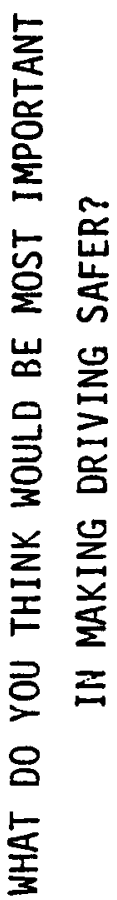

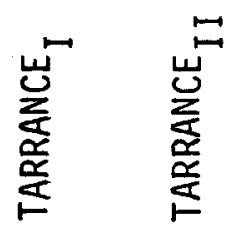

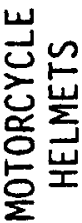

뚠

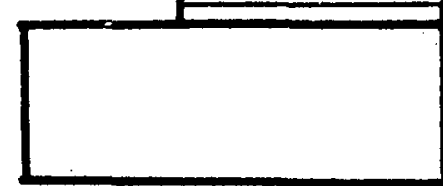

空导

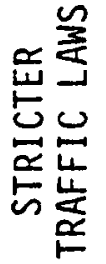

嶎

خu

w!

空岕
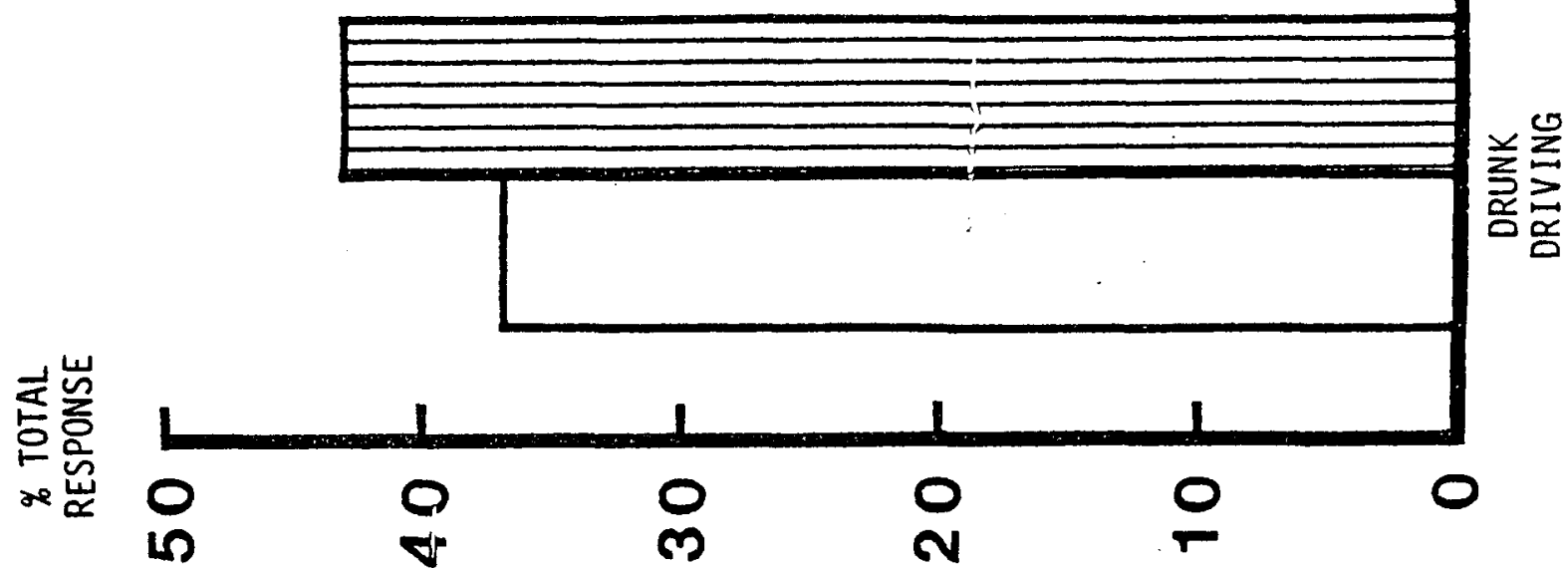
Figure 2 shows the main response categories and the percentage of the total number of responses in each of these categories. Examples of the specific responses are shown in Table 1 . These data add one additional issue to the data previously discussed and that is the reduction in speed. The difference in terms of percentages between these two sets of data may in part be that the first question related to highway safety issues directly and the second question relates more directly to the reasons for the decline in fatalities.

Another way of looking at the question of what the public perceives to be the major highway safety issues was addressed by the following question:

"In general, have your driving habits -- trip length, where you drive, when you drive, how you drive -- changed in the last year?"

Thirty percent of the respondents said "yes" and the other 70\% were "unsure". Less than $1 \%$ responded "no". Those who responded "yes" were then asked, "In what way have your driving habits changed?".

Table 2 provides examples of the responses given to this question. A companion question of "Why did your habits change" was also asked. The responses to this question, as shown in Table 3, reflect an awareness of drunk driving, safety belts and speed, as well as an additional nonsafety related item -- the economy.

In summary, the major highway safety related topics in 1983 which could have been influential in reducing the number of fatalities are:

\author{
Reduced speed \\ Awareness of drunk driving \\ Wearing safety belts
}

and the unrelated safety issue of the economy. 


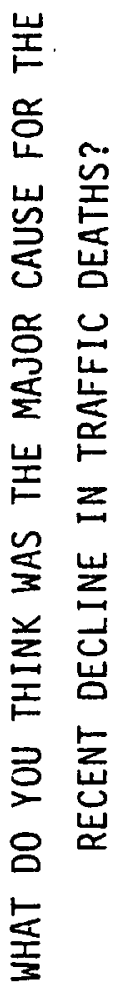

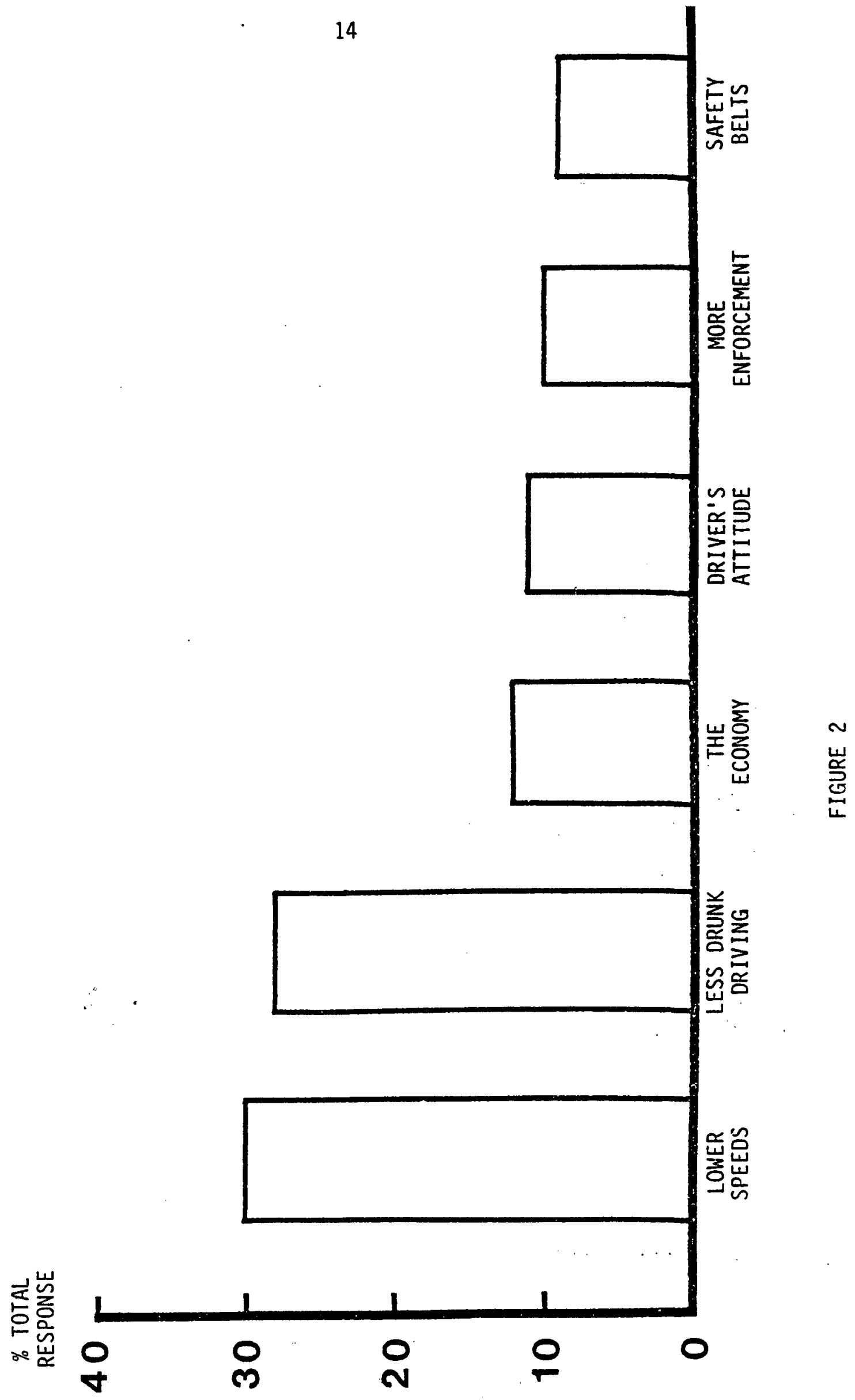


TABLE 1

\section{VERBATIMS - REASON FOR DECLINE IN AUTO FATALITIES}

Question 21:

The total number of auto fatalities and the number of auto fatalities per mile driven dropped significantly last year. What do you think was the major cause for this drop?

\begin{tabular}{lllll}
\hline $\begin{array}{l}\text { Geographic } \\
\text { Region }\end{array}$ & Age & Education & Sex & User \\
\hline Categories \\
\hline I/New England & & Some & Female/ & Frequent/ \\
States & $25-34$ & College & Home & Confirmed
\end{tabular}

"Getting the drunk drivers off the roads."

II/New York/

Nely Jersey

18-24 $\quad \begin{aligned} & \text { Some } \\ & \text { College }\end{aligned}$

\section{Female/ Sometime/ Works Likely}

"They raised the drinking age...that lowered the number of drunk drivers on the road."

IV/

South East $45-54$

Some

College

\section{Female/}

Home
Sometime/

Likely

"Wearing seat belts more...drivers more educated in driving better and obeying laws."

VII/

Plains

$$
\begin{array}{ll}
\text { 25-34 } & \text { Some } \\
\text { College }
\end{array}
$$

Male

Sometime/

Untikely

"Probably the increased enforciement of the 55 m.p.h...they recently had an awareness program going on in this area."

VI

Great Lakes
High Schoor

25-34

Graduate

\section{Female/ Infrequent/}

Home Confirmed

"Many people colildn't afford to travel as much last year as previous years because of economy."

V/

Great Lakes

$35-44$

Some

MaTe

Sometime/

Likely

"The high price of fuel...attitude of drivers... I had to drive to work... now I carpool." 
TABLE 1 (continued)

Question 21 continued:

\begin{tabular}{lllll}
\hline $\begin{array}{l}\text { Geographic } \\
\text { Region }\end{array}$ & Age & Education & Sex & $\begin{array}{l}\text { User } \\
\text { Categories }\end{array}$ \\
\hline $\begin{array}{l}\text { IIIf } \\
\text { Mid Atlantic }\end{array} 25-34$ & $\begin{array}{l}\text { High School } \\
\text { Graduate }\end{array}$ & $\begin{array}{l}\text { Female/ } \\
\text { Home }\end{array}$ & $\begin{array}{l}\text { Sonetime/ } \\
\text { Unlikely }\end{array}$ \\
"Driving more carefully because with insurance rates going up no one can \\
afford to have an accident."
\end{tabular}

IV/

High School

Female/ Sometime/

South East

$35-44$

Graduate Works Likely

"Perhaps because of increased exposure in that department by the media... increased education on the subject."

IV/

South East

$25-34$

\section{Some}

College
Female/

Home
Sometime/

Likely

"Probably stricter driving laws, the speed reduced and people are more aware now in wearing their seat belts and are using them more for children."

IV/

South East

\section{Some} $25-34$

Co1lege

Female/ Home
Infrequent/

Likely

"Because the police are out more to keep people from driving fast...tougher laws on drunk driving too." 
TABLE 2

VERBATIMS - HOW' DRIVING HABITS HAVE CHANGED

Question 45:

In what way have your driving habits changed in the last year?

\begin{tabular}{|c|c|c|c|c|}
\hline $\begin{array}{l}\text { Geographic } \\
\text { Region }\end{array}$ & Age & Education & Sex & $\begin{array}{l}\text { User } \\
\text { Categories }\end{array}$ \\
\hline $\begin{array}{l}\text { IV/ } \\
\text { South East }\end{array}$ & $45-54$ & $\begin{array}{l}\text { Less than } \\
\text { High School }\end{array}$ & Female & $\begin{array}{l}\text { Sometime/ } \\
\text { Likely }\end{array}$ \\
\hline
\end{tabular}

IV/

South East

Less than

$45-54$

High School

Male

Infrequent/

Confirmed

"Shorten trips...less gas being used."

II/New York/

New Jersey

$18-24$

Some

College

Infrequent/

Male

Confirmed

"I drive better... I'm more cautious and I drive defensively rather than offensively."

IXI

West coast

$25-34$
High School

Graduate
Female/ Sometime/

Home Likely

"I don't drive anymore... I kind of learned my lesson."

? V/

Great Lakes

$18-24$

Some

College

Female/

Infrequent/

"More responsible... more nlert."

III

Mid Atlantic

$35-44$

High School

Graduate

Female/

Home

Infrequent/

Likely

"Make sure I know what other people are doing, and not drink."

IV/

South East

Less than

High School

Male

Infrequent/

$18-24$

Likely

"Improved safefy habits...more conscious of people around me." 
TABLE 2 (continued)

Question 45 continued:

\begin{tabular}{|c|c|c|c|c|}
\hline $\begin{array}{l}\text { Geographic } \\
\text { Region }\end{array}$ & Age & Education & Sex & $\begin{array}{l}\text { User } \\
\text { Categories }\end{array}$ \\
\hline Mid South & $25-34$ & $\begin{array}{l}\text { Less than } \\
\text { High School }\end{array}$ & Male & $\begin{array}{l}\text { Sometime/ } \\
\text { Unlikely }\end{array}$ \\
\hline \multicolumn{5}{|c|}{ "I took a defansive course class and now I'm more aware of my driving." } \\
\hline $\begin{array}{l}\text { IV/ } \\
\text { South East }\end{array}$ & $55-64$ & $\begin{array}{l}\text { College } \\
\text { Graduate }\end{array}$ & $\begin{array}{l}\text { Female/ } \\
\text { Home }\end{array}$ & $\begin{array}{l}\text { Sometime/ } \\
\text { Likely }\end{array}$ \\
\hline
\end{tabular}

$\begin{array}{lllll}\text { IV/ } & 65 \& & \text { Less than } & \text { Female/ } & \text { Frequent/ } \\ \text { South East } & \text { over } & \text { High School } & \text { Home } & \text { Confirmed }\end{array}$

"More nervous to get on the highway with so many cars rushing off and on the freeway." 


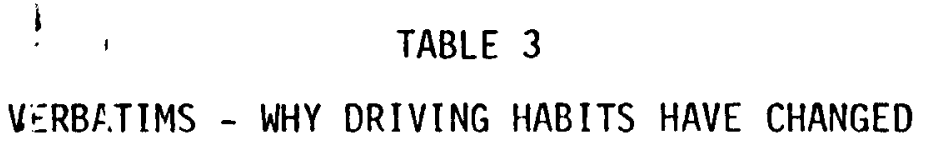

Question 46:

And why did you change your driving habits?

\begin{tabular}{|c|c|c|c|c|}
\hline $\begin{array}{l}\text { Geographic } \\
\text { Region }\end{array}$ & Age & Education & Sex & $\begin{array}{l}\text { User } \\
\text { Categories }\end{array}$ \\
\hline $\begin{array}{l}\text { VII } \\
\text { Mid South }\end{array}$ & $55-64$ & $\begin{array}{l}\text { Some } \\
\text { College }\end{array}$ & Male & $\begin{array}{l}\text { Frequent/ } \\
\text { Confirmed }\end{array}$ \\
\hline \multicolumn{5}{|c|}{$\begin{array}{l}\text { "The company has changed by cutting back on our car trips and traveling... } \\
\text { since business is slow we do a lot of work by phone calls now." }\end{array}$} \\
\hline $\begin{array}{l}\text { XI } \\
\text { North West }\end{array}$ & $25-34$ & $\begin{array}{l}\text { Some } \\
\text { College }\end{array}$ & $\begin{array}{l}\text { Female/ } \\
\text { Works }\end{array}$ & $\begin{array}{l}\text { Frequent/ } \\
\text { Confirmed }\end{array}$ \\
\hline
\end{tabular}

VI/

Mid South $18-24$

Some

"I changed because I had to drive the long distance to school.".

IV/
South East $\quad 45-54 \quad \begin{aligned} & \text { Less than } \\ & \text { High School Male }\end{aligned}$ Infrequent/
Confirmed

IV/ High School Female/ Frequent/

South East 25-34 Graduate Works Confirmed

"Because we have a new baby in our family."

IV/

South East $18-24$

Less than

High School

Infrequent/

"It just happened... you just get.older and wiser and reach that certain age when you look out for yourself and family."

VI/

Mid South

$55-64$

Less than

High School

Male

Frequent'

Confirmed

"Less money so we don't drive as far and my wife just quit her job...cut down on driving." 
TABLE 3 (continued)

Question 46 continued:

\begin{tabular}{|c|c|c|c|c|}
\hline $\begin{array}{l}\text { Geographic } \\
\text { Region }\end{array}$ & Age & Education & Sex & $\begin{array}{l}\text { User } \\
\text { Categories }\end{array}$ \\
\hline $\begin{array}{l}\text { II/ Yew York/ } \\
\text { Pew Jersey }\end{array}$ & $18-24$ & $\begin{array}{l}\text { Some } \\
\text { College }\end{array}$ & Male & $\begin{array}{l}\text { Infrequent/ } \\
\text { Confirmed }\end{array}$ \\
\hline
\end{tabular}

"I had an accident and my ideas have changed... I grew up...my insurance went up."

II/:!el:! York/

New Jersey

$45-54$

High School

Female/

Intrequent/

Graduate

Works

Likely

"More wreckless drivers on the road...they are always trying to pass you and honk at you when they are behind you."

I I/llew York/

iiew Jersey'
High School

$45-54$

\section{Graduate}

$\begin{array}{ll}\text { Female/ Infrequent/ } \\ \text { Works } & \text { Likely }\end{array}$

"A11 the programs about drinking and driving have influenced me...they are good."

I/New England

States

25-34

High School
Graduate

Female/

Sometime/

Home

Likely

"I'm more aware...the whole public is aware... I don't know but when I have my kids with me I drive a lot safer."

VI/

Mid South

$35-44$

High School

Graduate $\begin{array}{ll}\text { Female/ } & \text { Sometime/ } \\ \text { Home } & \text { Unlikely }\end{array}$

"I think it's just the fear of watching for the other guy." 
SELF-REPORT OF SAFETY BELT USAGE

Using the five point scale for self-report of safety belt use developed in Tarrance I, a comparison can be made of the percentage of the population that falls into each of these categories in Tarrance $I$ and Tarrance II. Figure 3 shows this comparison. There is a $38 \%$ increase between Tarrance I and Tarrance II in the percentage of people who classify themselves as confirmed-users, or an absolute increase of $9 \%$... This data compares favorably with the data from another question in the survey (separated from each other by about 40 questions) which asked:

"Which of the following best describes your use of safety belts compared to a year ago?"

1. I use them significantly more than I used to. $\frac{9 \%}{10 \%}$

2. I use them slightly more than I used to. $\frac{10 \%}{72 \%}$

3. I use them about the same. $\underline{72 \%}$

4. I use them slightly less than I used to. $3 \%$

5. I use them significantly less than I used to. $4 \%$

6. Don't know/no answer.

$2 \%$

This indicates that the percentage that increased their usage was approximately twice that of those individuals who said they decreased their usage. The validity of these responses is substantiated when the data is cross-referenced by the five self-report of use categories which were developed in Tarrance I (see Figure 4). The reasons given for both increased and decreased use of the safety belt are shown in Tables 4 and 5 . The reasons for increased use reflect the increased public awareness of the positive value of safety belts. The reasons for the decreased use are essentially the same reasons that are always given. Unfortunately, some things never change.

The demographic characteristics of the five categories of users are shown in Table 6 . 

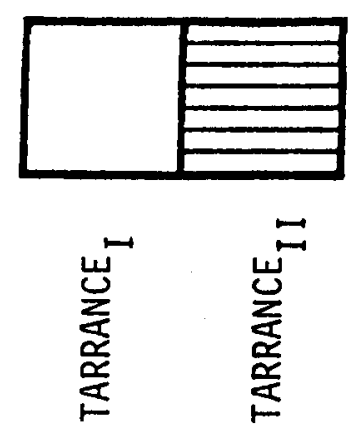

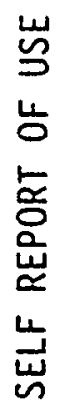

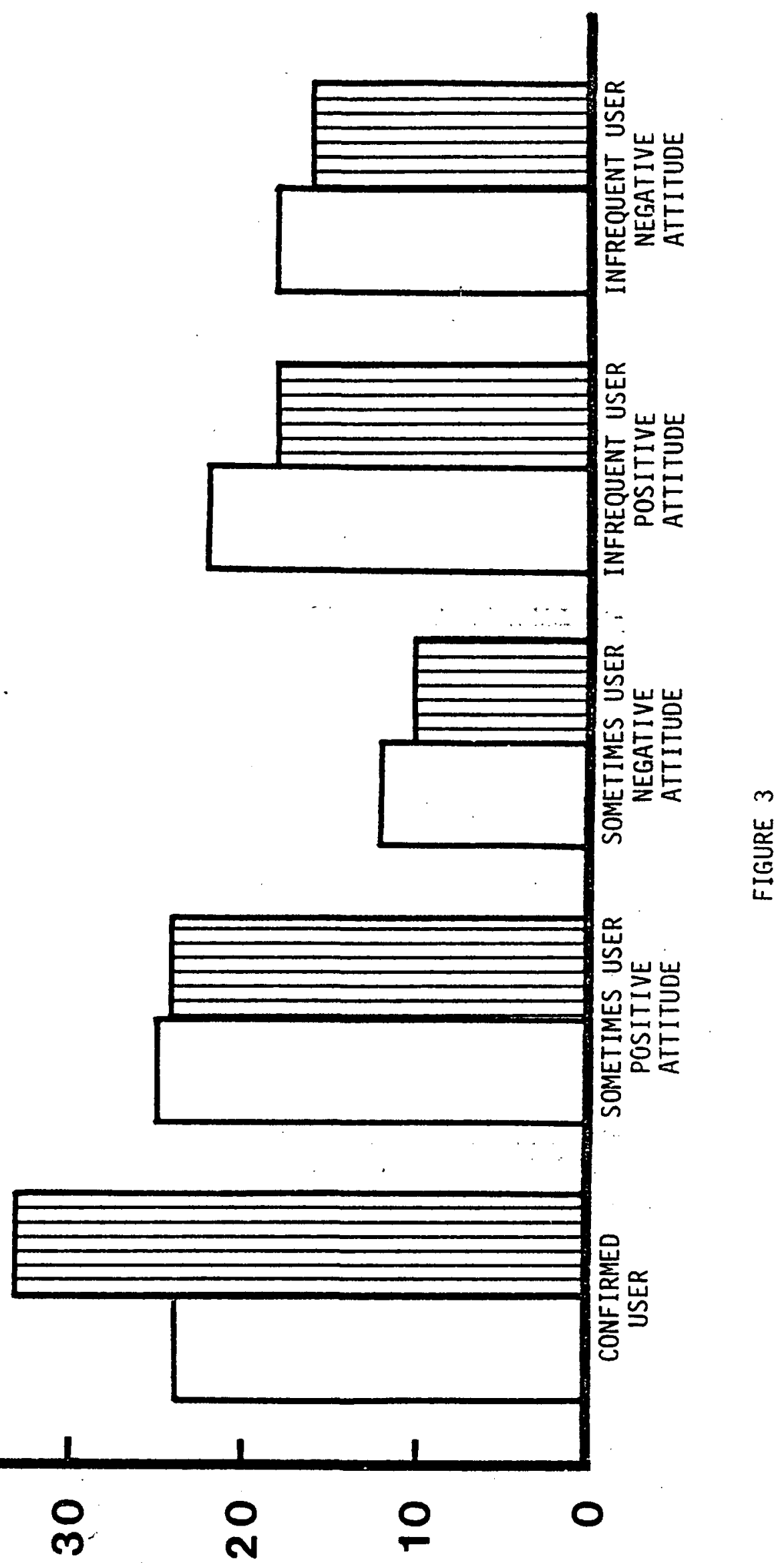




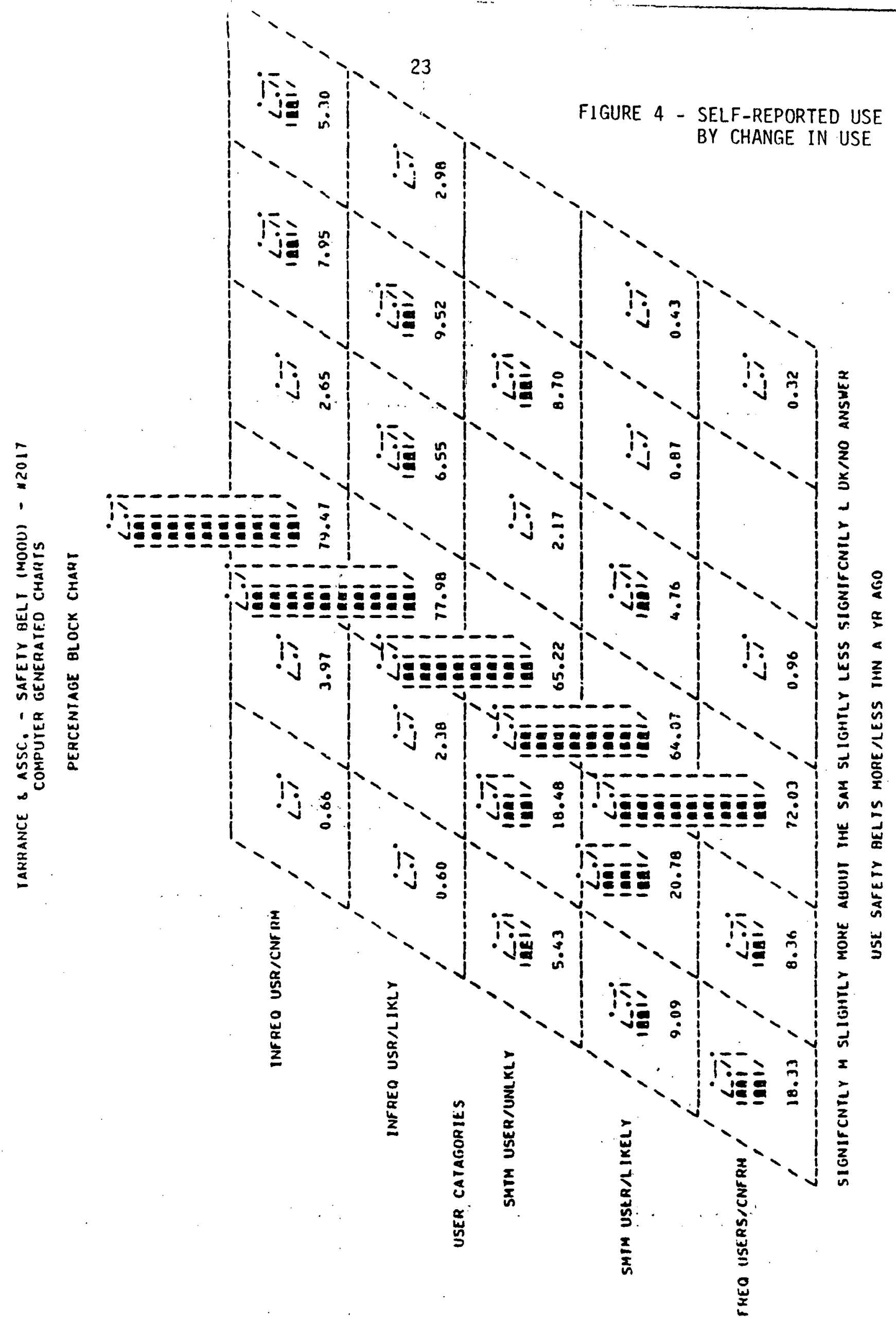


TABLE 4

VERBATIMS - WHY USE SAFETY BELTS MORE

Question 53:

And what are one or two reasons why you are using safety belts more than you used to?

\begin{tabular}{lllll}
\hline $\begin{array}{l}\text { Geographic } \\
\text { Region }\end{array}$ & Age & Education & Sex & Categories \\
\hline IX' & & $\begin{array}{l}\text { College } \\
\text { Graduate }\end{array}$ & $\begin{array}{l}\text { Female/ } \\
\text { Works }\end{array}$ & $\begin{array}{l}\text { Frequent/ } \\
\text { Confirmed }\end{array}$ \\
West Coast & $35-44$ & and &
\end{tabular}

"I have a smaller car and I don't feel as safe in it as I would in a bigger car."

IV/

South East

25-34

Some

College

Male

Frequent/

Confirmed

"The campaigns going on here...ads on TV...we are deluged with it and makes you think about. wearing belts more... it makes you more aware of what can happen."

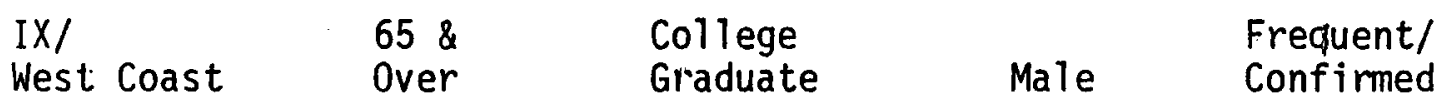

"I have a newer car and it is better equipped and lots easier to use so I use the seat belts now more than ever."

IV/

South East

$35-44$

High School

Female/

Frequent/

"More people are speeding and drinking and too many careless people on the road...never feel sorry to use your seat belt."

V/

Great Lakes
High School
Graduate

$18-24$

Female/
Works

Frequent/

Confirmed

"Had a son and it really makes you more cautious in everything you do because you want to be around to bring up your family... it realiy shocked me when it happened."

IV/

South East

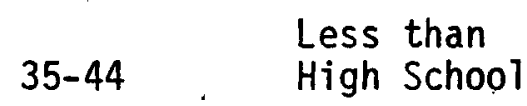

Male

Infrequent/

"The truth is, after I did this survey a year ago, it made me more aware of the fact I should try to wear them more." 
TABLE 4 (continued)

Question 53 continued:

\begin{tabular}{|c|c|c|c|c|}
\hline $\begin{array}{l}\text { Geographic } \\
\text { Region }\end{array}$ & Age & Education & Sex & $\begin{array}{l}\text { User } \\
\text { Categories }\end{array}$ \\
\hline $\begin{array}{l}\text { V/ } \\
\text { Great Lakes }\end{array}$ & $25-34$ & $\begin{array}{l}\text { Some } \\
\text { College }\end{array}$ & $\begin{array}{l}\text { Female/ } \\
\text { Home }\end{array}$ & $\begin{array}{l}\text { Sometime/ } \\
\text { Unlikely }\end{array}$ \\
\hline
\end{tabular}

III/

Mid Atlantic

25-34

High School

Sometimej

Graduate Male Likely

"I have witnessed a few accidents that people were hurt in and this could have been prevented with the use of seat belts."

VII/

Plains
High School

Graduate

$\begin{array}{ll}\begin{array}{l}\text { Female/ Sometime/ } \\ \text { Home }\end{array} & \begin{array}{l}\text { Likely } \\ \text { Likel }\end{array}\end{array}$

"Because I was in a wreck and got a knee injury...I don't think I would have it if I had on my seat belt."
V/

Great Lakes
College

Graduate
Sometime/

Likely

"If I do have an accident I don't want me or my wife to go through the windshield." 
TABLE 5

VERBATIMS - WHY USE SAFETY BELTS LESS

Question 54:

And what are one or two reasons why you are using safety belts less than you used to?

\begin{tabular}{lllll}
\hline $\begin{array}{l}\text { Geographic } \\
\text { Region }\end{array}$ & Age & Education & Sex & $\begin{array}{l}\text { User } \\
\text { Categories }\end{array}$ \\
\hline $\begin{array}{l}\text { X/ } \\
\text { North West }\end{array}$ & $25-34$ & $\begin{array}{l}\text { Some } \\
\text { College }\end{array}$ & $\begin{array}{l}\text { Female/ } \\
\text { Works }\end{array}$ & $\begin{array}{l}\text { Sometime/ } \\
\text { Unlikely }\end{array}$ \\
$\begin{array}{l}\text { "I am a short person and the seat belt comes across my neck and it's very } \\
\text { uncomfortable." }\end{array}$
\end{tabular}

VIII/

N.W. Plains

25-34 Come $\quad$ College

Female/ Sometime/

Home Likely

"Mostly because we changed cars... seat belts were hooked up in other car... these are lost in the seats.".

V/

Great Lakes
High School

Graduate
Sometime/

Male Likely

"I don't like them...only use when we have young children in the car."

$\begin{array}{llll}\text { IX/ } & \text { Some } & \begin{array}{l}\text { Female/ } \\ \text { Works }\end{array} & \begin{array}{l}\text { Infrequent/ } \\ \text { Confirmed }\end{array}\end{array}$

"I don't like to sit in the car with something restricting me... uncomfortable because I have had operations and other problems."

II/Ney: York/

New Jersey

\section{$35-44$}

College

Graduate
Female/

Works
Infrequent/

Confirmed

"When my children were younger I used to make everyone wear it... now that they're older, I've become lax about it... I guess I never really got into the habit."

VIII/

N.W. Plains
College

Graduate
Female/ Works
Infrequent/

Confirmed

"I'm always in a hurry to get in and out of my car...a iazy habit of not using them al though I believe in them." 
TABLE 5 (continued)

Question 54 continued:

\begin{tabular}{lllll}
\hline Geographic & Age & Education & Sex & $\begin{array}{l}\text { User } \\
\text { Region }\end{array}$ \\
\hline V/ & & $\begin{array}{l}\text { High School } \\
\text { Graduate }\end{array}$ & Male & $\begin{array}{l}\text { Infrequent/ } \\
\text { Likely }\end{array}$
\end{tabular}

"I used them more before my last auto accident... I had my belt on then and had a hell of a time getting it off so I just kind of stopped using them so much."

V/

Great Lakes

$18-24$

Some

College
Female/

Works
Infrequent/

Likely

"I don't like safety belts...I feel restrained in them and unsafe if the car should flip over and catch on fire."

VII/

Plains
High School

Graduate
Female/ Infrequent/
Works Likely.

"Well, it's just a short distance to work and people don't really think that anything is going to happen to them." 
TABLE 6 - SELF-REPORTED USE BY STANDARD FEDERAL ADMINISTRATIVE REGIONS

TARPANCE S ASSC. - SAFETY 3E-T :MOOOI - \$2017

DOT IPOL FREO USE SMTM USE SMTM USE INFREO U INFREO U

ICAELE RS/CUFRM R/LIKELY G/UNLKLY SR/LIKLY SR/CNFRM TOTAL

STINOAIZ FED. AOMNSTRETV REGIONS

\begin{tabular}{|c|c|c|c|c|c|c|}
\hline 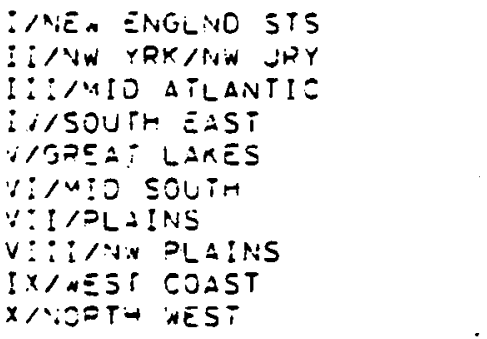 & $\begin{array}{ll} & 25.49 \\
: & 34.29 \\
: & 35.36 \\
: & 31.33 \\
: & 27.18 \\
: & 31.19 \\
: & 32.00 \\
: & 29.03 \\
: & 42.24 \\
: & 41.94\end{array}$ & $\begin{array}{l}19.61 \\
25.71 \\
24.24 \\
21.69 \\
27.69 \\
19.27 \\
26.00 \\
35.48 \\
25.00 \\
14.35\end{array}$ & $\begin{array}{r}7.84 \\
2.86 \\
7.07 \\
9.04 \\
10.25 \\
11.93 \\
16.00 \\
3.23 \\
12.07 \\
22.58\end{array}$ & $\begin{array}{l}25.47 \\
25.71 \\
19.18 \\
18.07 \\
17.75 \\
18.35 \\
10.00 \\
19.35 \\
11.21 \\
3.23\end{array}$ & $\begin{array}{l}21.57 \\
11.43 \\
14.14 \\
19.88 \\
16.92 \\
19.27 \\
19.00 \\
12.90 \\
9.48 \\
12.90\end{array}$ & $\begin{array}{r}51 \\
105 \\
99 \\
156 \\
195 \\
109 \\
50 \\
31 \\
116 \\
31\end{array}$ \\
\hline \multicolumn{7}{|l|}{ 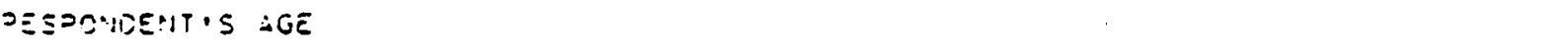 } \\
\hline 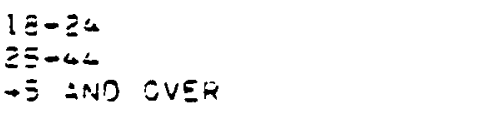 & $\begin{array}{l}22.80 \\
: \quad 33.44 \\
: \quad 37.54\end{array}$ & $\begin{array}{l}25.75 \\
27.29 \\
17.78\end{array}$ & $\begin{array}{r}10.69 \\
8.73 \\
10.44\end{array}$ & $\begin{array}{l}22 \cdot 14 \\
17.25 \\
16.48\end{array}$ & $\begin{array}{l}18.32 \\
15.28 \\
15.56\end{array}$ & $\begin{array}{l}131 \\
458 \\
364\end{array}$ \\
\hline \multicolumn{7}{|l|}{ ZESOSNEENTIS EDUCATION } \\
\hline 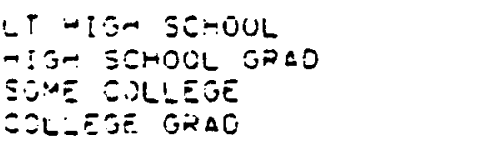 & 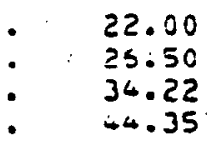 & $\begin{array}{l}17.00 \\
23.05 \\
25.06 \\
25.36\end{array}$ & $\begin{array}{r}15.00 \\
3.83 \\
9.89 \\
8.37\end{array}$ & $\begin{array}{l}19.00 \\
23.36 \\
15.35 \\
10.04\end{array}$ & $\begin{array}{l}27.00 \\
17.66 \\
13.69 \\
10.08\end{array}$ & $\therefore \begin{array}{l}100 \\
351 \\
263 \\
239\end{array}$ \\
\hline \multicolumn{7}{|l|}{ =ESइOOUDE::T'S SEX } \\
\hline $\begin{array}{l}\text { MLE } \\
\text { FEMALE/HOME } \\
\text { FEMALE/EMPLOYEO }\end{array}$ & $\begin{array}{l}29.72 \\
35.31 \\
34.96\end{array}$ & $\begin{array}{l}24.90 \\
23.58 \\
23.58\end{array}$ & $\begin{array}{r}10.88 \\
10.48 \\
5.50\end{array}$ & $\begin{array}{l}15.09 \\
17.03 \\
21.95\end{array}$ & $\begin{array}{l}18.62 \\
13.10 \\
13.01\end{array}$ & $\begin{array}{l}478 \\
229 \\
240\end{array}$ \\
\hline 2₹IMADV SOURCE OF FAMILY & DNCOME /C & & & & & \\
\hline $\begin{array}{l}\text { SLF-EMUL/NMITE } \\
\text { OLIE-COLLAR } \\
\text { VOT EMPLOYEO } \\
\text { OTHE? }\end{array}$ & $\begin{array}{l}37.23 \\
: \quad 23.91 \\
: \quad 33.33 \\
: \quad 32.43\end{array}$ & $\begin{array}{l}26.03 \\
23.48 \\
22.39 \\
22.32\end{array}$ & $\begin{array}{l}7.54 \\
10.43 \\
11.94 \\
11.71\end{array}$ & $\begin{array}{l}15.09 \\
24.35 \\
13.73 \\
19.32\end{array}$ & $\begin{array}{l}14.11 \\
17.83 \\
18.41 \\
13.51\end{array}$ & $\begin{array}{l}411 \\
230 \\
201 \\
111\end{array}$ \\
\hline 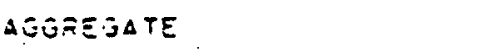 & 32.63 & 24.24 & 9.65 & 17.63 & 15.84 & 953 \\
\hline
\end{tabular}


TABLE 6 (continued)

TARDAICE O ASSC. - SAFETY SETT (MOOOI - \$2017

USER CATAGORIES

NOT $\triangle P P L$ FREO USE SMTM USE SMTM USE INFREO U INFREO U ICIELE RS/CVFRM P/LIKELY R/UNLKLY SR/LIKLY SR/CNFRM TOTAL

USE CMTSOURES

NOT ADELICACLE

F=E2 USEDS/CIVFNM

SUTY USE $=/$ LKELY

SYTY USED, UNLKLY

I.TF $\supset E O$ USR/LIKLY

IVF $=E$ USF/CIFFM

2SS/NHEEE. JO YOU LIVE

$\because \because a$ a dis etir

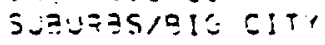

$\because y=$ SMAL $C: T Y$

T2N1/AILLACE

$\because \because$ i E EOUNTITY

:R1:00 Ar:S.*EF
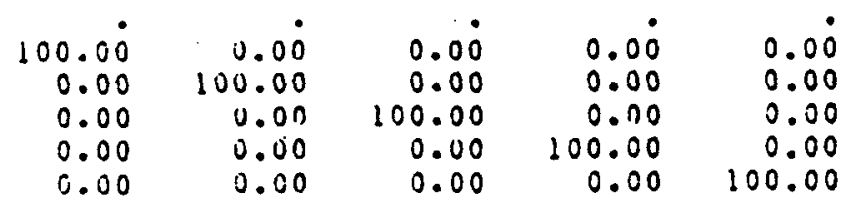

$31 i$
231
92
160
151

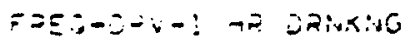

OEILY TO J HACHTH

SEJEEAL TUELYE

PEVEO

37.02
37.56
31.31
25.71
22.56
33.33

25.41
24.13
27.10
22.98
17.33
0.00

9.74

15.47

12.15

$12.09 \quad 15.22$

$15.42 \quad 15.32$

$24.84 \quad 14.29$

9.35

11.18

9.09

27.27

23.14

0.00

53.33

33.33

191

273

2! 4

151

121

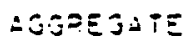

$\begin{array}{ll}29.02 & 22.26 \\ 32.24 & 31.59 \\ 33.97 & 22.53 \\ 32.63 & 24.24\end{array}$

8.29
10.93
9.71
9.65

17.62

10.38

173

$18.54 \quad 15.25 \quad 577$

$3 \overline{6} .63$

9.65

$17.63 \quad 15.84$

753 
Safety belt use is not a behavior which is sharply differentiated by traditional demographic and geographic characteristics of the population. There are some differences among demographic subgroups, but these relations are certainly less pronounced than those found in analyses of other behaviors and specific attitudes in the national population.

In general, safety belt wearing is positively related to socioeconomic status. Sixty-three percent of frequent users have attended or graduated from college, compared to $51 \%$ in the general population. Fortynine percent of frequent users are in the white-collar and self-employed occupation categories, while only about $37 \%$ of infrequent users are in those same upscale occupational divisions.

Frequent safety belt users tend to live in and around big cities, while infrequent users are more likely to be found in small towns and villages. The geographic pattern of seat belt usage across the country is somewhat mixed but in general we find the highest use of belts in the Pacific Northwest states and the lowest across the continent in the Northeast.

Some distinguishing patterns in seat belt use are apparent in the American adult population, but they are not the clear-cut demographic differences which lend themselves to full scale demographically-based targeting of specific groups for particular types of seat belt messages. In fact, the demographic and geographic data suggest that seat beit wearing is a behavior which, perhaps more than most behaviors, tends to cut across these usual lines of categorization. Seat belt use' is, in other words, a behavior not firmly rooted in the constellation of life circumstance and life change characteristics associated with standard demographic variables. 

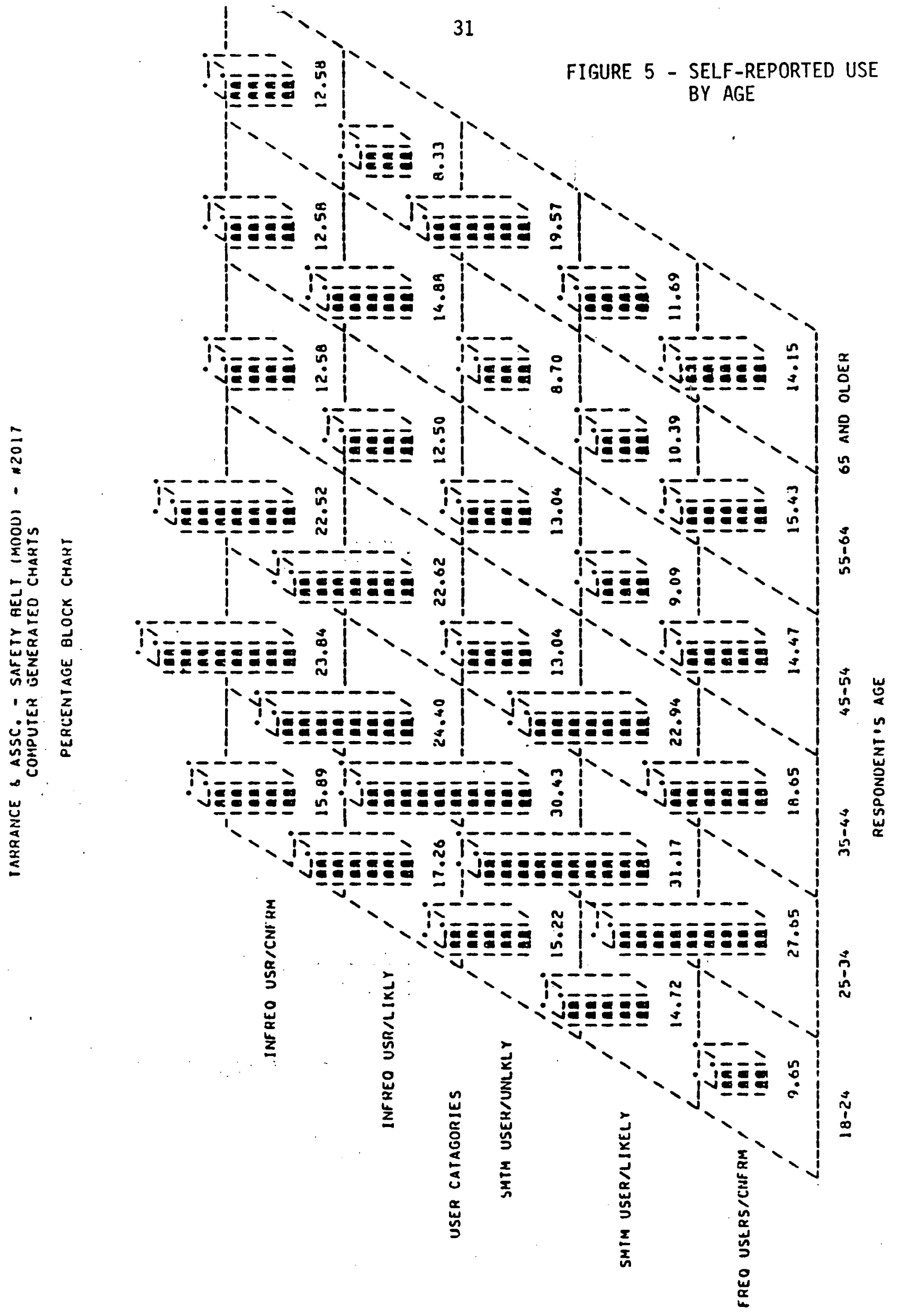
FIGURE 6 - SELF-REPORTED USE BY
EDUCATION

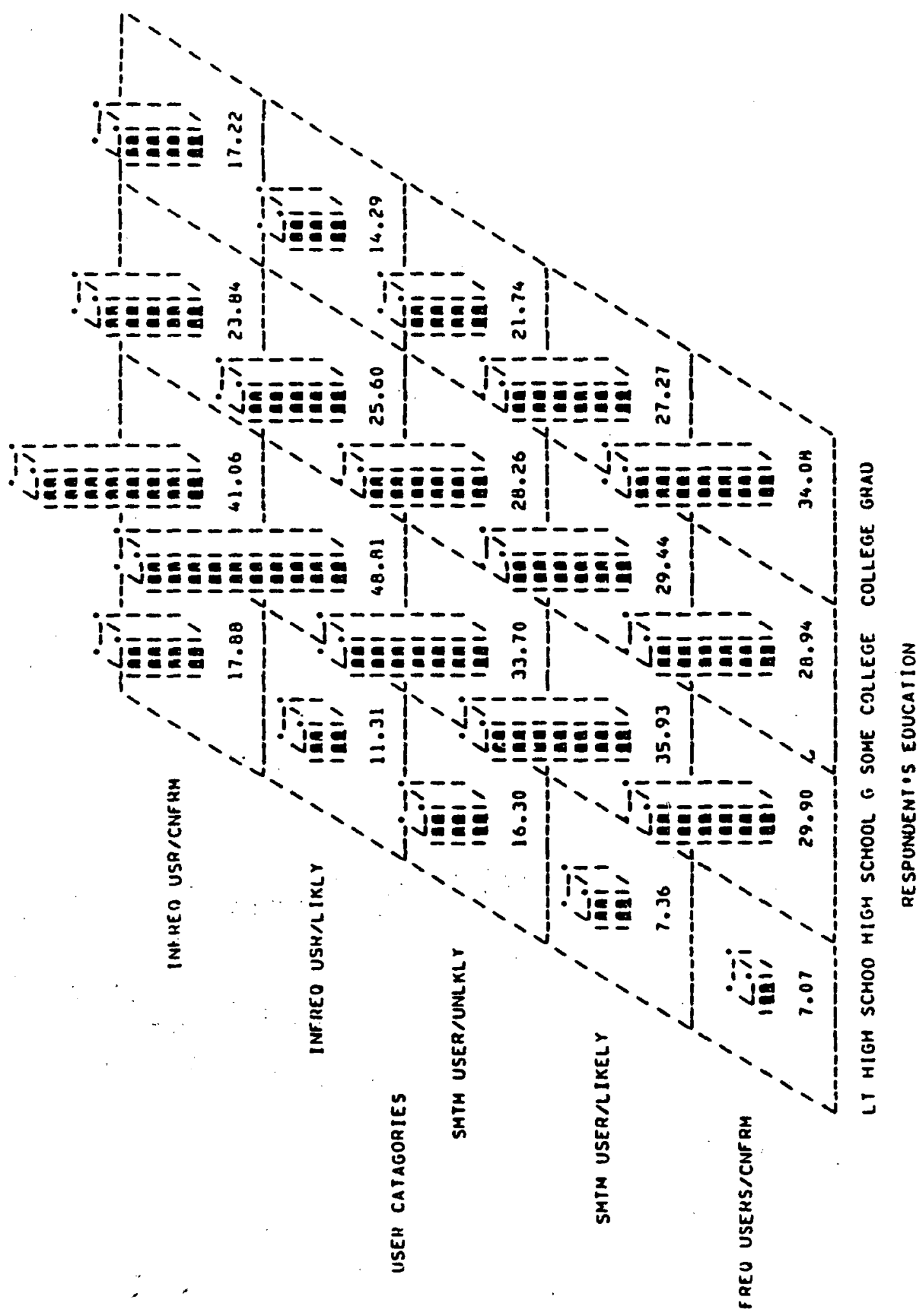




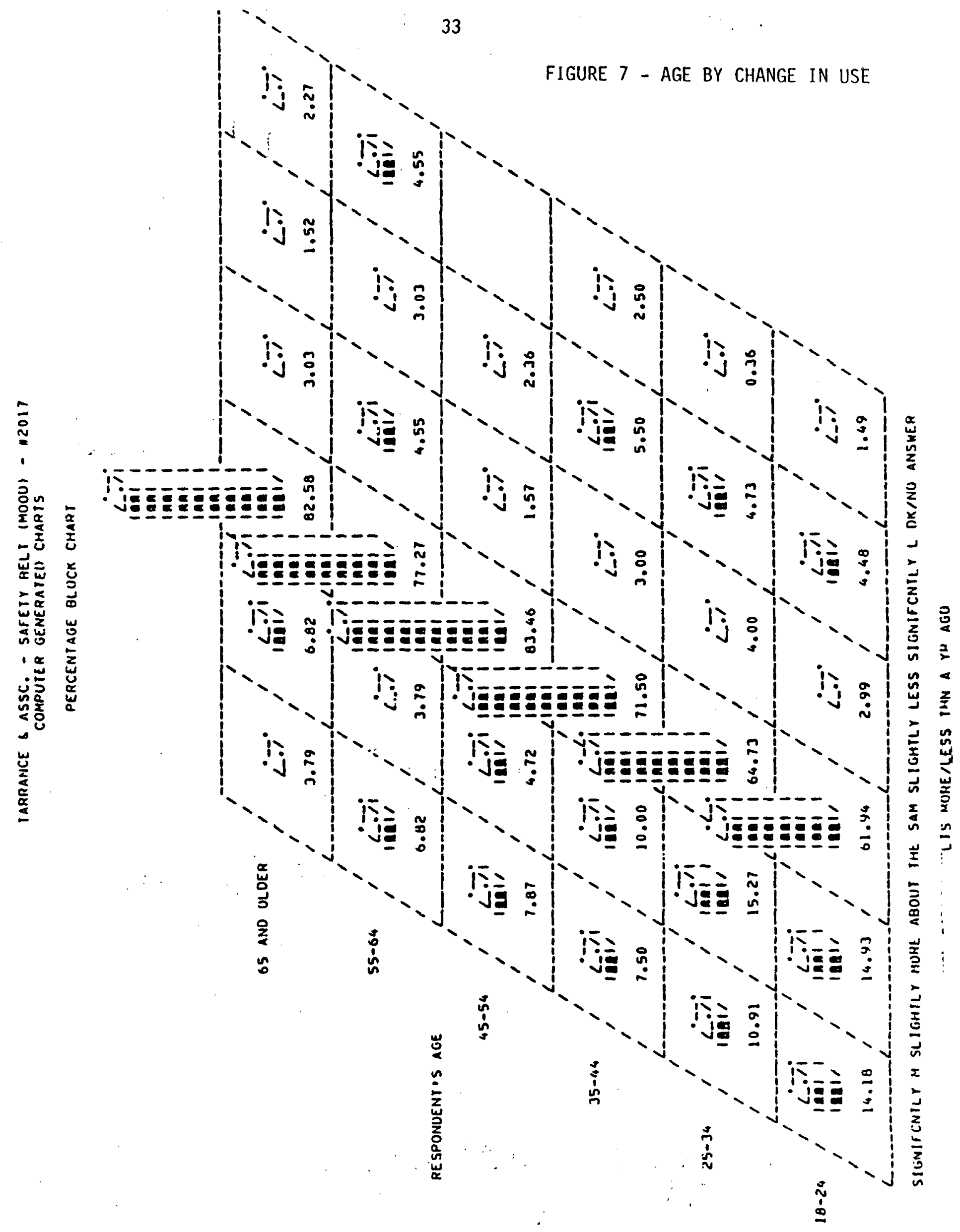


WHO DRINKS AND DRIVES?

Almost $63 \%$ of the respondents indicated that they do drink alcohol. Of those that do drink, $62 \%$ indicated that they drove within one hour of drinking at least once a year. Table 7 indicates the frequency of drinking and driving by the total population and by the population of those who drink and drive.

Twenty-five percent of those individuals who drive within one hour of drinking do so at least once a week, another $25 \%$ do so at least once a month. This translates to about 1 out of every 5 adults who drink and drive within one hour of drinking at least once a month. The demographic characteristics for those who drink and drive are presented in Table 8.

The demographic data on those who drink and drive confirms the same basic information that we have seen before regarding the characteristics of the drinking driver, namely, more males than females, and the 18-24 age group yielding the frequent drinkers/drivers, while those with less than a high. school diploma are underrepresented in the drinking/driving population. The most interesting demographic characteristic is that $29 \%$ of the confirmed non-belt wearers frequently drink and drive while only 18\% of the confirmed belt users frequently drink and drive.

This confluence of negatives (i.e., frequent drinking and driving and non-belt use) suggests that there may be a hard-core group of individuals who will be extremely resistant to changes in either area.

The sample sizes of this study do not permit a detailed investigation of either the attitudinal or demographic characteristics of this group. Thirty percent of those who drink and drive indicated that their frequency of driving within one hour of drinking had changed significantly over the last year. of those who had changed their frequency, $88 \%$ indicated that they had, in fact, reduced the frequency of drinking and driving. Table 9 presents the reasons people gave for changing the frequency of drinking and driving. Table 10 presents the data regarding where people drink and then drive and the associated frequency with which this occurs. 
TABLE 7

FREQUENCY OF DRIVING AND DRINKING BY DRIVERS/DRINKERS AND BY TOTAL SAMPLE

FREQUENCY OF DRINKING \& DRIVING

POPULATION WHO

$\frac{\text { DRINK \& DRIVE }}{\%}$
TOTAL

$\frac{\text { POPULATION }}{\%}$

$\begin{array}{lrr}\text { Daily } & 2 & 1 \\ 2-6 \text { times a week } & 9 & 4 \\ \text { Once a week } & 14 & 6 \\ \text { Every two weeks } & 8 & 3 \\ \text { Once a month } & 17 & 7 \\ 4-6 \text { times a month } & 19 & 7 \\ 1-2 \text { times a year } & 30 & 12 \\ \text { Never } & - & 60 \\ \text { Don't Know } & - & -\end{array}$


36

TABLE 8 - DEMOGRAPHICS BY FREQUENCY DRINK AND DRIVE

TARRANCE \& ASSC. - SAFETY BELT (MOOD) - \#2017

P23R/FREQNCY/ORIVE WITHIN I HR/DRINKNG/

OAILY TO SEVERAL NEVER
I/MONTH TMS/YR

TOTAL

STANOARO FED. ADMNSTRATV REGIONS

$\begin{array}{llllr}\text { I/NEW ENGLND STS } & 29.63 & 9.26 & 61.11 & 54 \\ \text { II/NW YRK/NW JRY } & 22.32 & 25.89 & 51.79 & 112 \\ \text { III/MIO ATLANTIC } & 21.90 & 14.29 & 63.81 & 105 \\ \text { IV/SOUTH EAST } & 18.39 & 12.07 & 69.54 & 174 \\ \text { V/GREAT LAKES } & 21.18 & 26.11 & 52.71 & 203 \\ \text { VI/MID SOUTH } & 20.54 & 14.29 & 65.18 & 112 \\ \text { VII/PLAINS } & 16.98 & 24.53 & 58.49 & 53 \\ \text { VIIINNW PLAINS } & 12.50 & 29.13 & 59.38 & 32 \\ \text { IX/WEST COAST } & 13.22 & 16.53 & 70.25 & 121 \\ \text { X/NORTH WEST } & 26.47 & 35.29 & 38.24 & 34\end{array}$

RESPONOENTIS AGE

$\begin{array}{lllll}13-24 & 28.36 & 23.88 & 47.76 & 134 \\ 25-44 & 24.63 & 22.53 & 52.84 & 475 \\ 45 \text { ANO OVER } & 11.51 & 13.81 & 74.68 & 391\end{array}$

RESPONDENTIS EDUCATION

$\begin{array}{lllll}\text { LT HIGH SCHOOL } & 6.25 & 12.50 & 81.25 & 112 \\ \text { HIGH SCHOOL GRAD } & 20.22 & 15.90 & 63.88 & 371 \\ \text { SOME COLLEGE } & 23.33 & 21.85 & 54.81 & 270 \\ \text { COLLEGE GRAD } & 22.27 & 24.70 & 53.04 & 247\end{array}$

RESPONDENTIS SEX

$\begin{array}{lrrrr}\text { MALE } & 32.06 & 21.04 & 46.89 & 499 \\ \text { FEMALE/HOME } & 5.76 & 13.58 & 80.66 & 243 \\ \text { FEMALE/EMPLOYEO } & 10.08 & 21.32 & 60.60 & 258 \\ \text { PRIMARY SOURCE OF FAMILY INCOME/C } & & & \\ & & & & \\ \text { SLF-EMPL/WHITE C } & 23.95 & 21.16 & 54.88 & 430 \\ \text { BLUE-COLLAR } & 20.99 & 23.46 & 55.56 & 243 \\ \text { NOTEMPLOYED } & 12.68 & 10.33 & 77.00 & 213 \\ \text { OTHER } & 16.67 & 20.18 & 63.16 & 114 \\ \text { AGGREGATE } & 20.00 & 19.30 & 60.70 & 1000\end{array}$


TABLE 8 (continued)

TARRANCE \& ASSC. - SAFETY BELT (MOOD) - \#2017

FREO-DRV-1 HR DRNKNG

DAILY TO SEVERAL NEVER I/MONTH TMS/YR TOTAL

USER CATAGORIES

NOT $\triangle P P L I C A B L E$

FREQ USERS/CNFRM

SMTM USER/LIKELY

SHTM USER/UNLKLY

INFREO USR/LIKLY

INFREQ USR/CNFRM

$\begin{array}{llll}18.01 & 18.97 & 63.02 & 311 \\ 18.51 & 25.11 & 56.28 & 231 \\ 17.39 & 21.74 & 00.87 & 92 \\ 20.24 & 16.07 & 63.69 & 168 \\ 29.14 & 12.58 & 50.28 & 151\end{array}$

P65/NHERE DO YOU LI.VE

\begin{tabular}{|c|c|c|c|c|}
\hline $\begin{array}{l}\text { IN A BIG CITY } \\
\text { SUEURZS/BIG CITY } \\
\text { IN A SMALL CITY } \\
\text { TOWN/VILLAGE } \\
\text { IN THE COUNTFY } \\
\text { DK/NO ANSWER }\end{array}$ & $\begin{array}{r}20.63 \\
20.91 \\
16.52 \\
20.00 \\
23.62 \\
0.00\end{array}$ & $\begin{array}{r}18.52 \\
22.65 \\
16.96 \\
20.59 \\
15.75 \\
0.00\end{array}$ & $\begin{array}{l}00.85 \\
56.45 \\
60.52 \\
54.41 \\
00.53 \\
100.00\end{array}$ & $\begin{array}{r}189 \\
287 \\
224 \\
170 \\
127 \\
3\end{array}$ \\
\hline FREQ-DHV-1 HR DRI & & & & \\
\hline $\begin{array}{l}\text { DAILY TO } 1 / M O N T H \\
\text { SEVERAL TMS/YR } \\
\text { NEVER. }\end{array}$ & $\begin{array}{r}100.00 \\
0.00 \\
0.00\end{array}$ & $\begin{array}{r}0.00 \\
100.00 \\
0.00\end{array}$ & $\begin{array}{r}0.00 \\
0.00 \\
100.00\end{array}$ & $\begin{array}{l}200 \\
193 \\
607\end{array}$ \\
\hline & & 19.3 & 00.70 & \\
\hline
\end{tabular}




\section{TABLE 9}

WHY PEOPLE CHANGED THEIR

.FREQUENCY OF DRINKING AND DRIVING

01. STRICTER D.W.I. LAWS - our stiffer drunk driving laws/ Taws are tougher/the new D.W.I. law that enforces 48 hours in jail/they are cracking down on laws for drunk drivers/

02. DON'T DRINK AS MUCH - I slowed down a lot on my drinking/ I have cut down a lot on my drinking/I consume much less alcohol/I am drinking less than I used to/

03. AM OLDER/MORE MATURE - I'm just getting older and thinking more about it/getting olcer, more cautious/as you get a little older, you slow ddwn/I am older and more mature/

04. MORE RESPONSIBILITY - more responsibility, I am thinking of the safety of myself and my family/I am more safety conscious, I've got a child now/care about my kids/

05. DON'T GO OUT AS MUCH - I don't go out as much, I usually drink at home/I don't go much anymore/married and didn't party as much/we don't go out very often/my social activities have become less/

06. MORE AWARE - I am more aware of the effects of drinking and driving/I'm more aware of it. being dangerous/awareness of drunk drivers/

07. GREATER DANGER OF ACCIDENTS - accidents I've heard about/ accidents around me/I realize the greater danger of accidents and I've become a better driver/more aware of accidents relating to drunk drivers/

08. DON'T DRINK AND DRIVE - don't drink and drive that much/I don't believe in drinking and driving/don't drink as often just before driving/I just have my wife drive me/I try not to drink when I'm driving/

09. AFRAID/DON'T FEEL SAFE - I'm afraid/drunken drivers scare me/don't feel safe anymore/

10. HAVE BEEN CAUGHT - got caught drinking and driving/I don't have a license and I have been arrested for drunken driving/I had a close call/

11. MONEY - my income has changed significantly/money/I can't afford to go out to clubs or parties as much anymore/

12. MEDICAL REASONS - I've got. medical problems... the doctor told me not to drink/I didn't drink last year because I was pregnant/ 
TABLE 9 (continued)

13. PERSONAL/BUSINESS REASONS - I've had a lot of personal probTems/I've got a steady girl friend and she tends to affect my judgment/if I take customers out or not, that makes it change

14. GO OUT MORE OFTEN - I go out more often/more social occasions/go to more social affairs and different functions/

15. DO IT MORE NOW - I do it more now/I've been drinking lately/

16: LIFE STYLE - because of my life style/changed my life style/

17. SAFETY EDUCATION - safety education, films seen at work/

18. OTHER

19. DON'T KNOW/NO ANSWER 
TABLE 10

PLACES PEOPLE DRINK AND THEN DRIVE

Bars/taverns

More than once a week........ ( $3 \%)$

About once a week...........2 (13\%)

One or two times a month......3 (15\%)

Less than once a month........4 (9\%)

One or two times a year.......5 (24\%)

Never..................6 (34\%)

Don't know/no answer......... (1\%)

Restaurants

More than once a week.........1

About once a week............2

One or two times a month......3

Less than once a month........4

One or two times a year......5

Never.................6

Don't know/no answer.........

Cocktail lounges

More than once a week.........1

About once a week............2

One or two times a month......3

Less than once a month.......4

One or two times a year.......5

Never..................6

Don't know/no answer.........7

Other people's homes

More than once a week......... 1

About once a week............

One or two times a month.......3

Less than once a month.......4

One or two times a year.......5

Never.....................6 6

Don't know/no answer......... 7 
TABLE 10 (continued)

Private party held in a public

place, such as a hotel or banquet hall.

More than once a week.........

About once a week............

One or two times a month.......3

Less than once a month........4

One or two times a year........5

Never....................6

Sporting events

More than once a week..........

About once a week............2

$(1 \%)$

One or two times a month.......3

Less than once a month........4

One or two times a year.......5

Never..................6

(17\%)

(72\%)

Don't know/no answer.........7

Drinking in my car

More than once a week.........1

About once a week............2

One or two times a month.......3

Less than once a month........4

One or two times a year........5

Never.................6 6

Don't know/no answer......... 
When asked the question:

"Have you personaliy made any efforts to reduce the number of times you have driven after drinking too much in the past 6 months?"

The response was split down the middle with as many people saying "yes" as saying "no".

For those who responded "yes"; they were then asked what they had done and if what they had done was something they just recently started to do. Table 11 presents these results.

Four behaviors were mentioned by $15 \%$ or more of the population, and of those, three are behaviors which the majority have just recently started to use.

These are:

- Limit the number of drinks.

- Had someone else drive home.

- Don't drink alcoholic beverages.

The majority of the people apparently have been using the approach of stopping their drinking at a predetermined time and have been using this approach for some time.

When asked "How can you tell when you have had too much to drink to drive safeiy?", the most frequent response was "the number of drinks". However, only $12 \%$ of the respondents mentioned this. The next two most frequent responses (each mentioned by $8 \%$ of the respondents) were "weaving/staggering" and "general behavior". The majority of the respondents didn't provide any response to this question. Table 12 provides a complete set of responses to this question. With regard to the issue of how to tell when you have had too much to drink and drive, and what can be done to reduce the number of occurrences of drinking and driving, a great deal of work still remains to be done. 
TABLE 11

WHAT HAVE YOU DONE TO CHANGE DRIVING AND DRINKING HABITS

\section{WAT HAVE YOU DONE TO CHANGE ORIVING AND DRINKING HABITS}

\begin{tabular}{|c|c|c|c|c|c|c|}
\hline & & $\begin{array}{l}\text { What have } \\
\text { Mentioned }\end{array}$ & $\begin{array}{l}\frac{\text { you done }}{\text { Not }} \\
\text { Mentioned }\end{array}$ & $\begin{array}{l}\text { Have } \\
\text { to } \\
\text { Rece } \\
\text { Yes }\end{array}$ & $\begin{array}{l}\text { you } 5 \\
\text { do t } \\
\text { ntty } \\
\text { No }\end{array}$ & $\begin{array}{l}\text { arted } \\
\text { is } \\
\text { Don } t \\
\text { Know } \\
\end{array}$ \\
\hline a) & $\begin{array}{l}\text { Don't drink alcoholic } \\
\text { beverages }\end{array}$ & $19 \%$ & $81 \%$ & $\underline{15 \%}$ & $7 \%$ & $79 \%$ \\
\hline b) & Limit the number of drinks & $38 \%$ & $62 \%$ & $\underline{26 \%}$ & $\underline{12 \%}$ & $62 \%$ \\
\hline c) & Eat food & $5 \%$ & $95 \%$ & $3 \%$ & $5 \%$ & $93 \%$ \\
\hline d) & $\begin{array}{l}\text { Drink some alcoholic } \\
\text { beverages and some: } \\
\text { non-alcoholic beverages }\end{array}$ & $3 \%$ & $97 \%$ & $1 \%$ & $4 \%$ & $95 \%$ \\
\hline e) & $\begin{array}{l}\text { Stop drinking at a } \\
\text { predetermined time }\end{array}$ & $15 \%$ & $85 \%$ & $7 \%$ & 9\% & $84 \%$ \\
\hline f) & $\begin{array}{l}\text { Had someone else drive } \\
\text { home }\end{array}$ & $36 \%$ & $64 \%$ & $23 \%$ & $14 \%$ & $63 \%$ \\
\hline g) & $\begin{array}{l}\text { Took a taxi/public } \\
\text { transportation }\end{array}$ & $3 \%$ & $97 \%$ & $2 \%$ & $4 \%$ & $95 \%$ \\
\hline h) & $\begin{array}{l}\text { Test self for own alcohol } \\
\text { level }\end{array}$ & $1 \%$ & $99 \%$ & $=$ & $3 \%$ & $97 \%$ \\
\hline i) & Stayed overnight & $4 \%$ & $96 \%$ & $4 \%$ & $3 \%$ & $94 \%$ \\
\hline j) & Drank coffee & $4 \%$ & $96 \%$ & $3 \%$ & $3 \%$ & $94 \%$ \\
\hline k) & Diluted drinks & $1 \%$ & $99 \%$ & $1 \%$ & $3 \%$ & $97 \%$ \\
\hline 1) & other (SPECIFY) & $13 \%$ & $87 \%$ & $\underline{11 \%}$ & $5 \%$ & $84 \%$ \\
\hline
\end{tabular}


TABLE 12

INDICATIONS - CAN'T DRINK AND DRIVE SAFELY

How can you tell when you have had too much to drink to drive safely?

Not

Mentioned

a) Number of drinks

$\begin{array}{r}12 \% \\ 5 \% \\ \begin{array}{r}58 \% \\ \hline\end{array} \quad 92 \% \\ \hline\end{array}$

d) Personality change, such as very talkative, loud, aggressive, very quiet, etc.

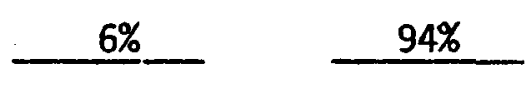

e) Can't stand up

$3 \%$

$97 \%$

f) Sleepy

$5 \%$

$95 \%$

g) General behavior

$8 \%$

$92 \%$

h) Passed out

$1 \%$

$99 \%$

i) Can just tell (nothing specific)

8\%

$92 \%$

j) Can't tell

$6 \%$

$94 \%$

k) Other (SPECIFY) 
FIGURE 8 - FREOUENCY DRINK/DRIVE BY REDUCE DRINK/DRIVE BY AGE
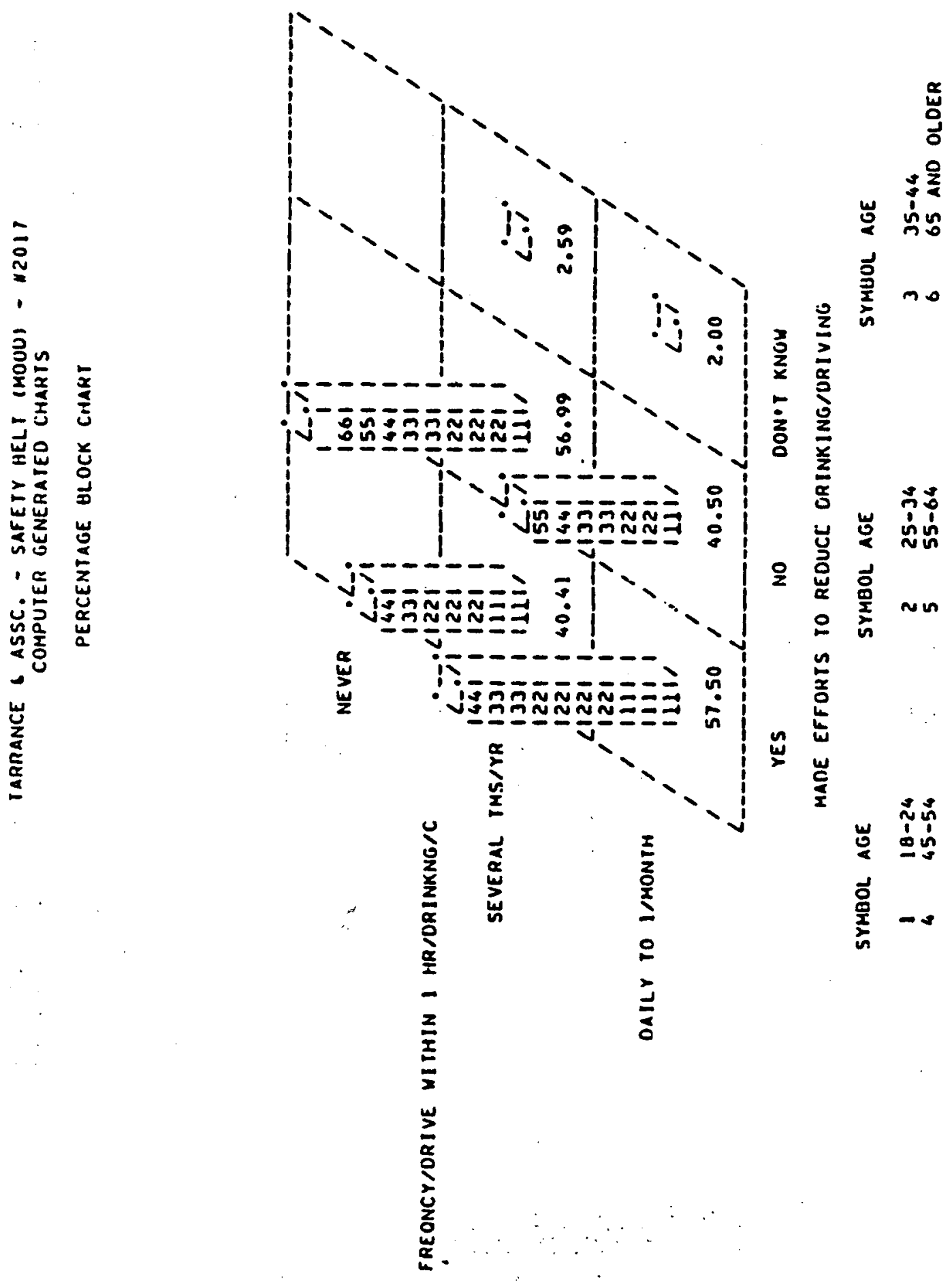
FIGURE 9
-FREQUENCY DRINK/DRIVE BY
EDUCATION

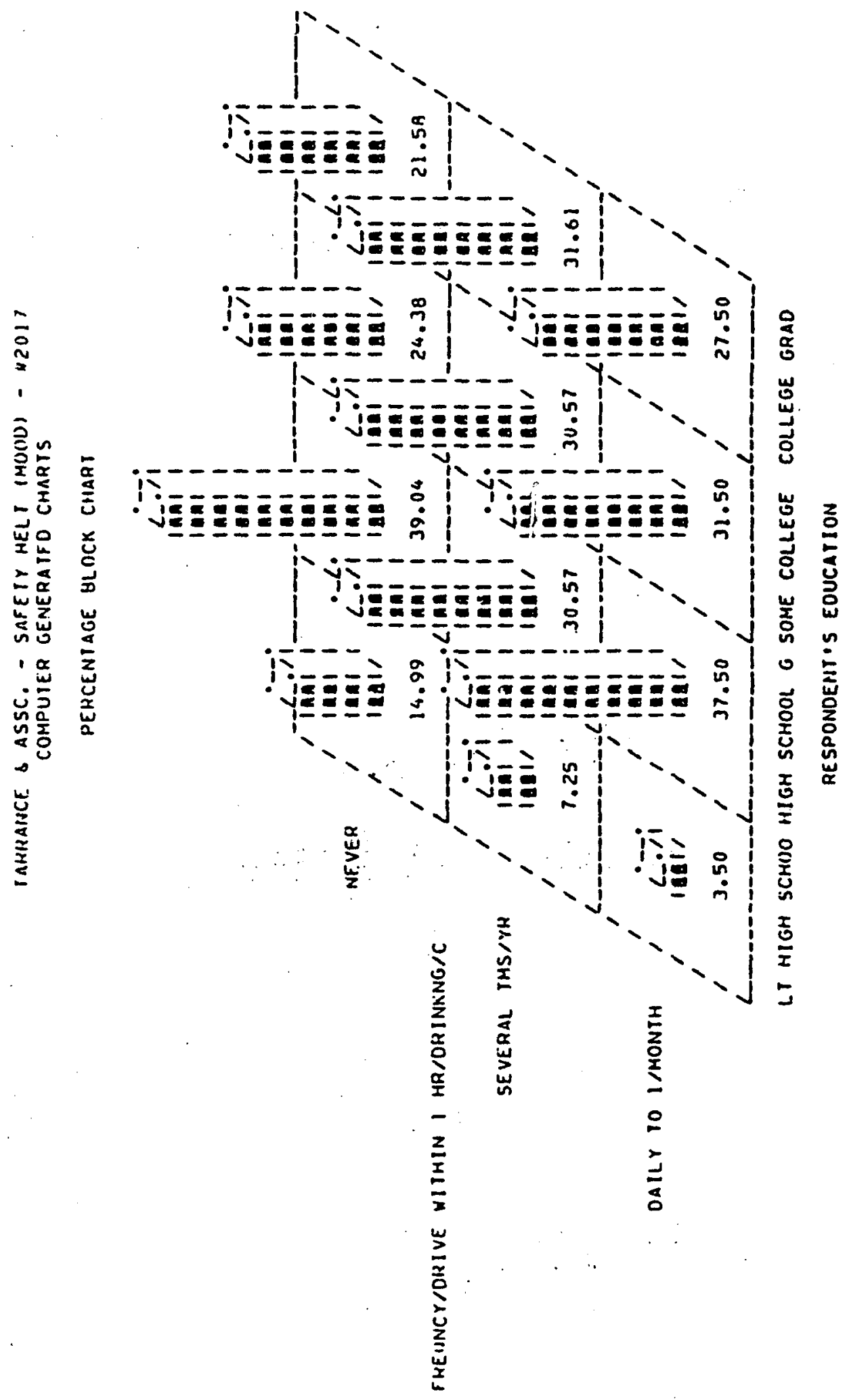




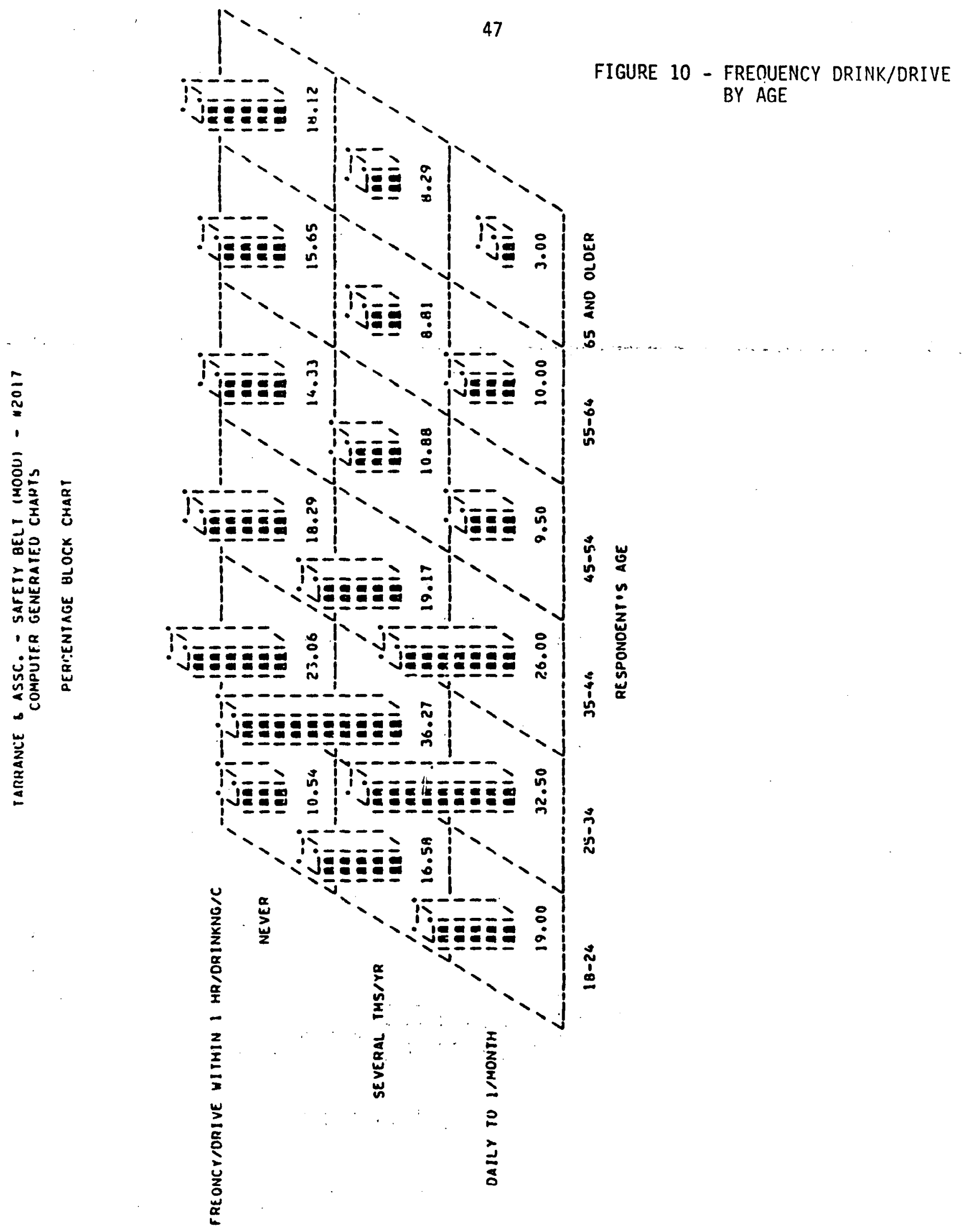


FIGURE 11 - FREQUENCY DRINK/DRIVE BY STOP STATED TIME BY AGE

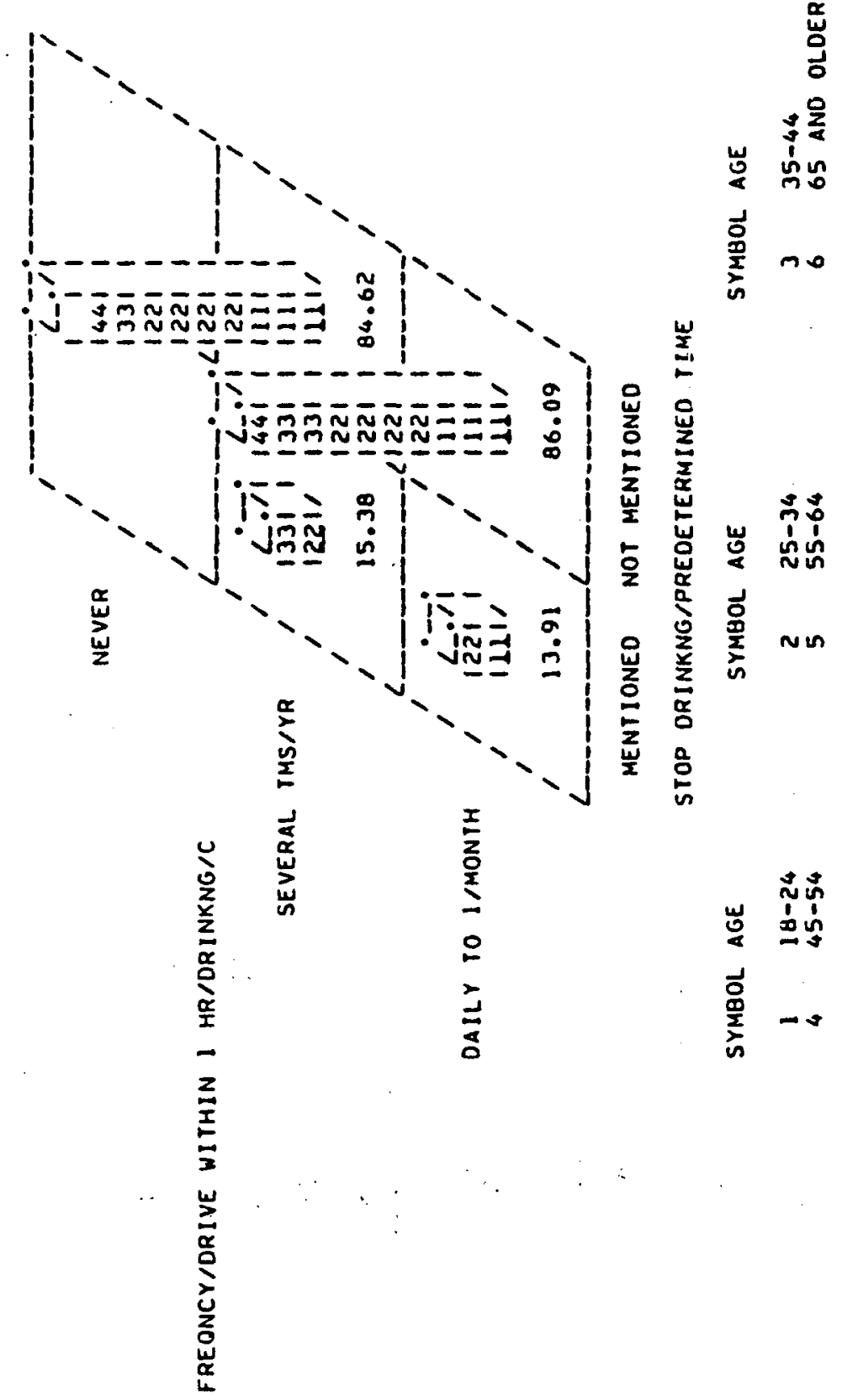


FIGURE 12 - FREQUENCY DRINK/DRIVE BY NO ALCOHOL BY AGE
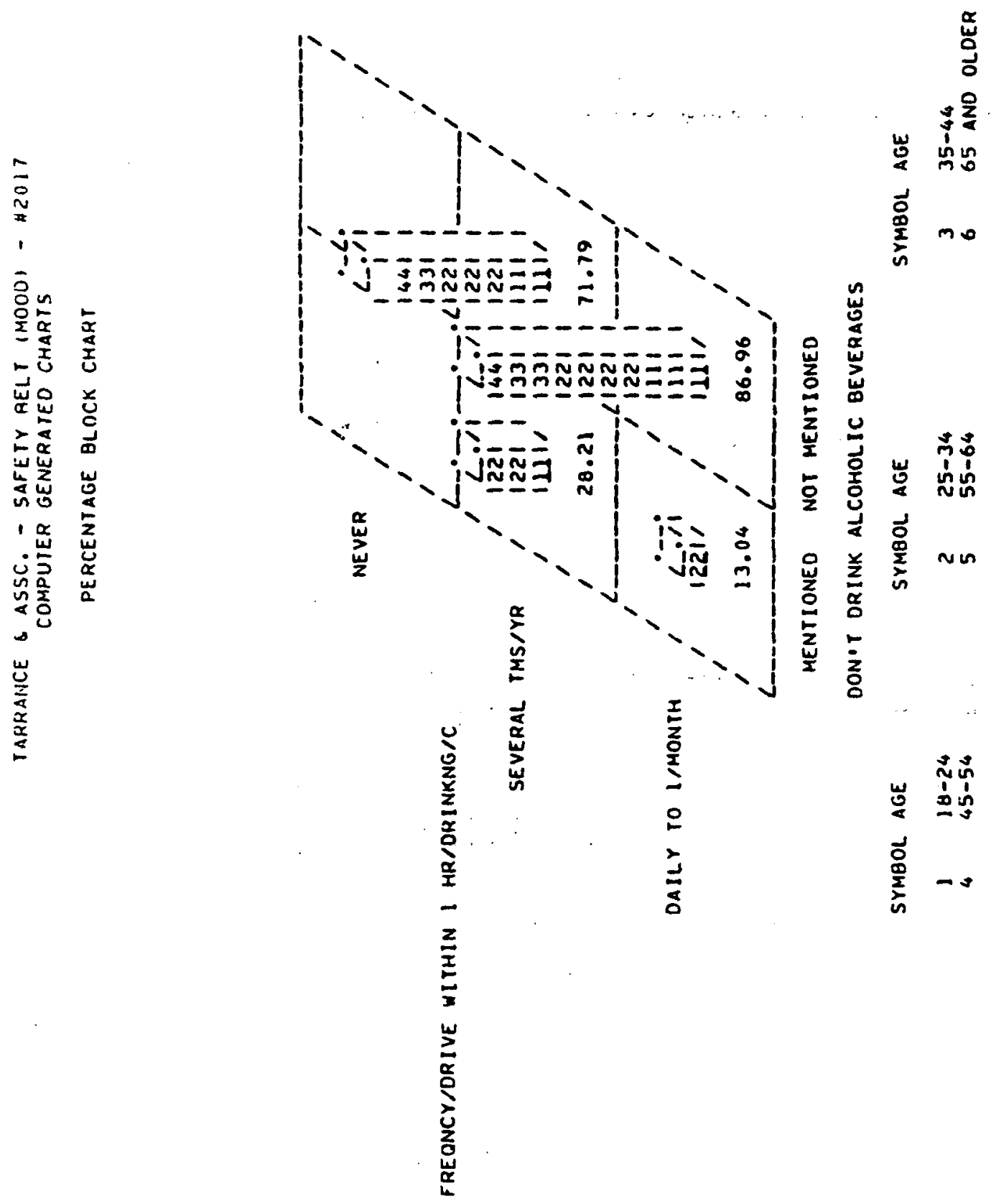
FIGURE 13 - FREQUENCY DRINK/DRIVE BY HAVE OTHER DRIVE BY AGE

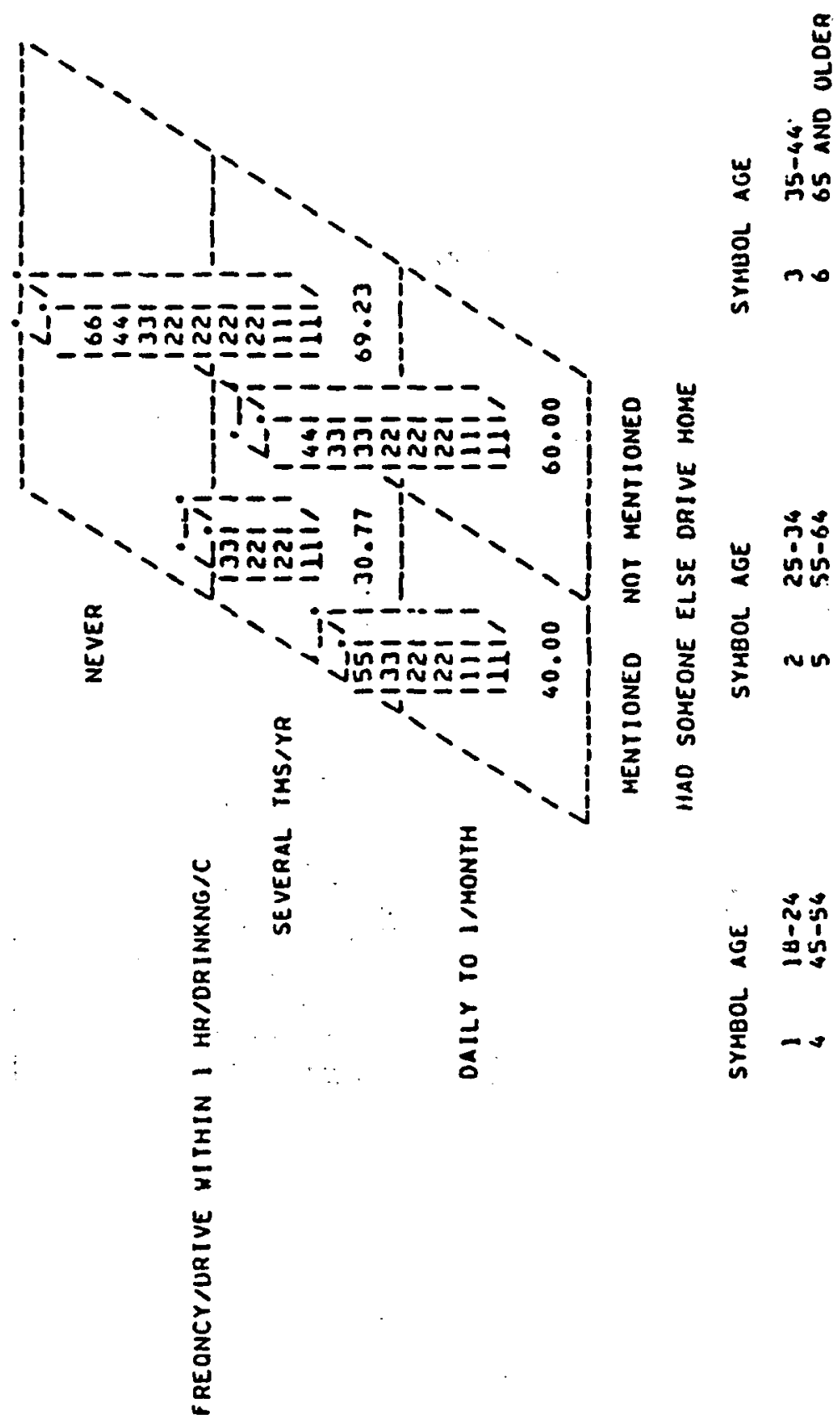


FIGURE 14 - FREQUENCY DRINK/DRIVE BY LIMIT DRINKS BY AGE

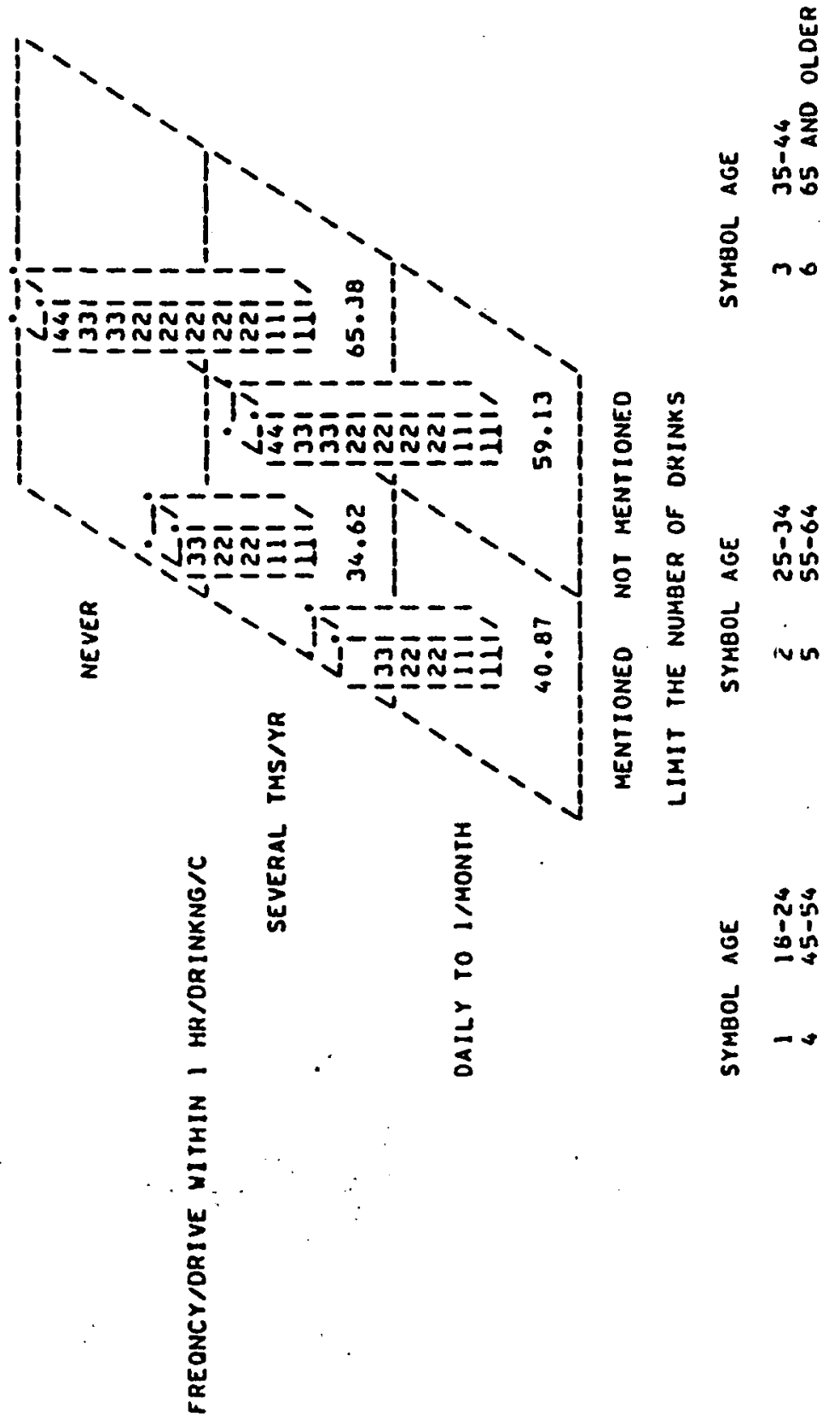


TABLE 13

VERBATIMS - WHY HAS DRINKING/DRIVING CHANGED

Question 26:

And what are one or two reasons why you feel that your frequency of drinking within one hour of driving has changed significantly over the past year?

\begin{tabular}{|c|c|c|c|c|}
\hline $\begin{array}{l}\text { Geographic } \\
\text { Region }\end{array}$ & Age & Ellucation & Sex & $\begin{array}{l}\text { User } \\
\text { Categories }\end{array}$ \\
\hline $\begin{array}{l}\text { V/ } \\
\text { Great Lakes }\end{array}$ & $45-54$ & $\begin{array}{l}\text { H'lgh School } \\
\text { Graduate }\end{array}$ & $\begin{array}{l}\text { Female/ } \\
\text { Home }\end{array}$ & $\begin{array}{l}\text { Infrequent/ } \\
\text { Likely }\end{array}$ \\
\hline
\end{tabular}

VII

Mid South

35-44. Some

Female/ Infrequent/

Home... Likely

"I go to more social affairs and different functions."

IXI

West Coast
College

Graduate
Female/ Sometime/

Works Likely

"I can't afford to go out to clubs or parties as much anymore."

\begin{tabular}{|c|c|c|}
\hline $\begin{array}{l}\text { I I/New York/ } \\
\text { New Jersey }\end{array}$ & $35-44$ & $\begin{array}{l}\text { College } \\
\text { Graduate }\end{array}$ \\
\hline
\end{tabular}

"I realize the greater danger of accidents and I've become a better driver."

VIII/

N.W. Plains

$25-34$

College

Graduate

Female/ Infrequent/

Works Likely

"I got married and had a baby and stay home more...also, the drunk driving laws are so much stricter than they used to be."

V/ Great Lakes $25-34$

Some

College
Frequent/

Male
Confirmed

"Well, the first thing is that I'm older and more mature... I have a lot more responsibility thrown on me than I did before... I guess the other thing is that I've had some bad experiences in the past with friends drinking and driving." 
TABLE 13 (continued)

Question 26 continued:

\begin{tabular}{lllll}
\hline $\begin{array}{l}\text { Geographic } \\
\text { Region }\end{array}$ & Age & Education & Sex & User \\
\hline $\begin{array}{l}\text { II/New York/ } \\
\text { New Jersey }\end{array}$ & $18-24$ & $\begin{array}{l}\text { Some } \\
\text { College }\end{array}$ & Male & $\begin{array}{l}\text { Infrequent/ } \\
\text { Likely }\end{array}$
\end{tabular}

"I slowed down a lot on my drinking... if I'm really drunk now, my friends will drive... I'm 24 now and I guess I'm getting more aware than when I was 18."

II/New York/

New Jersey
Some

College
Female/ Frequent/ Works Confirmed

"Police is after everyone and MADD people are really working hard to catch them... I don't want to be the one." 


\section{DRIVING PATTERNS}

Data was collected on various aspects of the respondents' driving patterns. This data is presented in Tables 14 through 24 . While the data is presented in a number of different forms, the most interesting cuts are those regarding frequency, drinking and driving, and self-report of safety belt use. Both the confirmed non-wearers and the frequent drinkers tend to drive more per year than the sample as a whole. This suggests that the two groups whose behavior tends to increase their risk are also on tile road more, so that their exposure is a lot higher than the general public's. This set of relations with regard to increased exposure, holds when mileage data is broken out for both an average weekday and weekend.

These two segments of the population appear to travel by themselves more than the general public. The data regarding the number of people in the car on the last trip of over 200 miles indicates that both groups had the highest percentage of one person trips for their respective categories.

Table 22 shows no differences between. these two groups in terms of change in family driving patterns in the past year and Table 23 provides the same data for the individual respondent. Neither of the tables shows any significant differences between the two high risk groups and the general population data. This impact may be a result of the large percentage of no change for both tables.

The only table. which shows a discrepancy in terms of reported behavior of the two righ risk groups is Table 24 which describes the use of their car during the last Thanksgiving through New Year holiday season. The frequent drinkers drive more while the infrequent wearers drove less than the general public.

The reasons for more frequent or less frequent use for all respondents are presented in Tables 25 and 26. 
TABLE 14 - DEMOGRAPHICS BY MILES DRIVEN PAST YEAR

TARRANCE \& ASSC. - SAFETY BELT (MOOD) - $\$ 2017$

P3SR/MILES DRIVEN/LAST I2 MONTHS/C

$$
\begin{array}{rrrrrr}
0-1.000 & 1,200-5,6,000-10 & 11,000-1 & 16.000 & \text { S OK/NO AN } & \\
000 & .000 & 5,000 & \text { OVER } & \text { SWER TOTAL }
\end{array}
$$

STMIOANE FED. LOMNSTRATV PEGIONS

I/NEW ENGLNO STS

IINW YOK.NNW JFY

IIITMIO DTLANTIC

IVISOUTH EAST

VAPEAT LANES

VI/MIO SOUTH

$V I I / D L A I N S$

V:T:INAN PLATNS

IXINEST COAST

$X$ NAOOTH AEST

FESPONDENT'S AGE

$13-24$

$2 \equiv-44$

-5 IND OVER

14.31

15.18

$1+.29$

18.39

9.85

6.25

5.60

25.00

17.36

11.75

$\begin{array}{ll}14.81 & 25.73 \\ 27.58 & 18.75 \\ 25.71 & 16.19 \\ 14.94 & 17.24 \\ 19.70 & 21.18 \\ 22.32 & 15.18 \\ 22.64 & 22.54 \\ 21.88 & 21.88 \\ 22.31 & 16.53 \\ 29.41 & 117.65\end{array}$

18.52

14.29

21.90

15.09

15.76

25.00

18.37

9.38

15.70

23.53

13.66

9.47

19.40

19.53
25.32

23.13

17.89

18.16

20.15
20.00

20.00

14.93
25.74

25.74

18.52

17.86

16.19

26.44

23.15

25.00

22.64

9.38

22.31

17.65

$$
\begin{array}{r}
7.41 \\
6.25 \\
5.71 \\
6.30 \\
10.34 \\
6.25 \\
7.55 \\
12.50 \\
5.79 \\
0.00
\end{array}
$$

17.65

3.73

7.37

8.18

136

475

RESPOYOENTIS EDUCATION

LT HIGA SCHOOL
HIUA SCHOOL GR:O
SOME COLLEGE
SOLLEGE GRAD
FESPCNDENTIS SEX
MALE

FEMALE/HOME

FEMALE/EMPLOYED

25.00

15.30

10.37

2.10

25.00

22.54

19.25

10.07

16.98

20.74

3.57
16.44

19.84

20.24

20.37

16.07

19.41

24.44

14.29

8.63

4.31

4.45

112

23.08

24.29

23.45

29.22

15.03

29.63

20.24

15.23

18.99

4.94

33.47

7.41

2.61

13.58

10.08

479

243

258

PEIMARY SOURCE OF FAMILY INCOME/C

SLF-EMOL/WHITE C
OUJE-COLLAE
INOT EMPLOYEO
OTHER
LGOREGATE

$11.11 \quad 20.16$

27.70

7.89

27.70

19.30

20.16

16.90

16.67

20.47

18.93

7.04

24.56

26.28

23.46

12.21

13.70

17.70

21.60

6.28

6.17

8.45

10.53

7.20

430

243

213

114

13.50

21.30

18.70

1000 
TABLE 14 (continued)

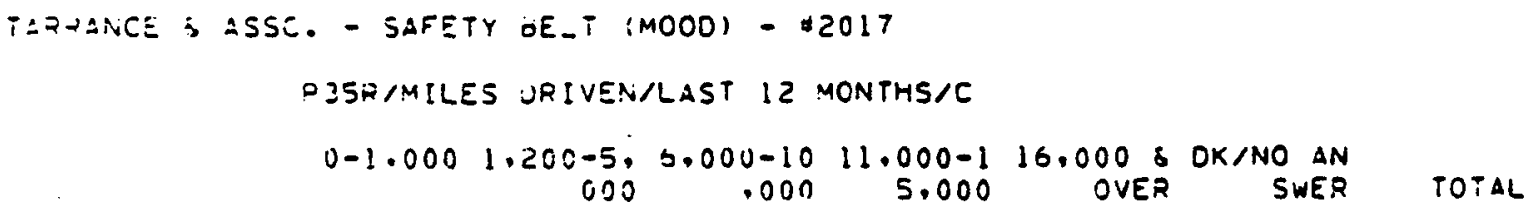

TOTAL

7.97

16.45

7.73

13.45

13.25

$$
\begin{aligned}
& 25.45 \\
& 22.51 \\
& 25.04 \\
& 13.45 \\
& 15.34
\end{aligned}
$$

22.19

10.45

1.7 .39

10.45

15.23

16.08
18.61
26.09
17.86
15.09

21.86

19.48

14.13

19.64

31.79

5.47

6.49

0.52

7.14

7.95

2S5/ARFEE OO YOU IIVE

$\because \because 24: 5:-4$

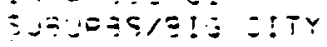

:! - SALL CITY

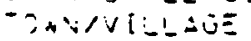

$\because T-E$ SJUH:T-Y

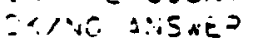

$\therefore \leq-\infty 0$

20.11

20.71

$20.7 \mathrm{e}$

13.35

$\therefore-.12$

22.35

52.23

12.60

33.33

13.34

$2 ! .45$

13.64

20.00

13.39

0.00

$$
\begin{aligned}
& 19.05 \\
& 16.72 \\
& 19.20 \\
& 14.71 \\
& 13.70 \\
& 33.33
\end{aligned}
$$

20.11
23.34
18.75
29.59
26.77
0.00

3.99

5.23

8.04

8.24

5.51

33.33

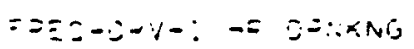

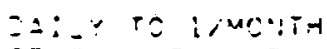

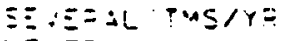

5.50
7.25

15.00

27.40

21.00

2.73

23.00
20.73

21.42

17.30

14.99

31.00

19.69

3.50

4.15

200

$\therefore \therefore=\Xi \because-\square$

13.50

21.30

15.70

17.70

21.60

7.20
189

287

224

170

127

3


TABLE 15 - DEMOGRAPHICS BY MILES DRIVEN WEEKDAYS

TARRANCE \& ASSC. - SAFETY BELT (MOOD) - $\$ 2017$

P36R/MILES DRIVEN/AVE. WEEK DAY/C

0-10 12-29 30-69 70 \& OVE DK/NO AN

SWER TOTAL

STANOARD FED. ADMNSTRATV REGIONS

I/NEW ENGLND STS

II/NW YRK/NW JRY

III/MID ATLANTIC

IV./SOUTH EAST

VIGREAT LAKES

VI/MID SOUTH

VII/PLAINS

VIII/NW PLAINS

IX/WEST COAST

$X / N O R T H$ WEST
25.93

36.61

25.71

20.69

24.63

25.00

26.42

46.88

24.79

32.35
20.37

14.29

16.19

19.54

19.21

19.64

22.64

25.00

20.66

25.47
31.48

18.75

30.48

21.84

23.15

25.89

13.21

9.38

24.79

20.59
16.67

27.68

25.71

35.63

30.54

25.00

30.19

18.75

27.27

20.59
5.56

2.68

1.90

2.30

2.46

4.46

7.55

0.00

2.48

0.00
54

112

105

174

203

112

53

32

121

34

RESPONDENT'S AGE

$18-24$

$25-44$

45 AND OVER
24.63

22.74

31.97
18.66

20.00

18.67
27.61

24.21

20.20
27.61

31.16

24.55
1.49

1.89

4.60
134 475 391

RESPONDENT'S EOUCATION

LT HIGH SCHOOL

HIGH SCHOOL GRAD

SOME COLLEGE

COLLEGE GRAD
37.50

26.15

25.56

23.48
24.11

18.06

13.70

25.10
14.29

26.15

23.70

21.86
17.86

28.03

33.33

27.13
6.25

1.62

3.70

2.43
112

371

270

247

RESPONDENT'S SEX

MALE

FEMALE/HOME

FEMALE/EMPLOYED
17.43

42.80

29.07
17.64

20.58

21.32
24.25

20.58

23.26
37.88

13.17

23.26
2.81

2.88

3.10
499

243

258

PRIMARY SOURCE OF FAMILY INCOME/C

SLF-EMPL/WHITE C

BLUE-COLLAR

NOT EMPLOYED

OTHER

AGGREGATE
22.56

22.22

42.25

21.93

26.60
17.91

22.63

14.55

25.32

19.30 $\cdot$

22.56

28.81

18.31

21.93

34.42

25.10

19.25

27.19

23.10

28.10

2.56

430

243

213

114

2.63

2.90

1000 
TABLE 15 (continued)

tarrance \& assc. - SafeTy Belt (MOOD) - \#2017

P36R/MILES DRIVEN/AVE. WEEK DAY/C

0-10 $\quad 12-29 \quad 30-6970 \&$ OVE OK/NO AN

TOTAL

USER CATAGORIES

NOT $\triangle P P L I C A B L E$

FREQ USERS/CNFRM

SMTM USER/LIKELY

SMTM USER/UNLKLY

INFREQ USR/LIKLY

$\begin{array}{rrr}25.08 & 21.22 & 22.51 \\ 32.03 & 15.15 & 21.21 \\ 28.26 & 18.48 & 28.26 \\ 26.79 & 16.67 & 26.19 \\ 19.87 & 24.50 & 19.21\end{array}$

27.97
29.00
22.83
28.57
31.79

3.22
2.60
2.17
1.79
4.64

$31 i$

231

92

168

INFREQ USR/CNFRM

PS5/WHERE DO YOU LIVE

IN A BIG CITY
SUBURGS/BIG CITY
IN A SMALL CITY
TOWN NVILLAGE

I.V THE COUNTRY

DK/NO ANSWER

$\begin{array}{rrrr}30.69 & 17.58 & 17.46 & 29.10 \\ 25.09 & 18.47 & 27.87 & 26.48 \\ 29.91 & 19.75 & 24.11 & 24.55 \\ 29.41 & 19.82 & 20.59 & 27.06 \\ 14.96 & 22.05 & 22.05 & 38.58 \\ 0.00 & 33.33 & 33.33 & 0.00\end{array}$

3.17

2.09

2.68

4.12

2.36

33.33
189

287

224

170

127

3

FREQ-DRV-1 HR DRNKNG

DAILY TO I/MONTH SEVERAL TMS/YR NEVER

21.00

27.46

28.17

14.50

26.50

22.28

23.32

36.50

26.42

25.86

1.50

0.52

4.12

200

193

507

$26.60 \quad 17.30$

23.10

28.10

2.90

1000

AGGREGATE

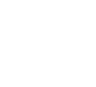


59

TABLE 16 - DEMOGRAPHICS BY MILES DRIVEN WEEKENDS

TARRANCE \& ASSC. - SAFETY BELT (MOOD) - \#2017

P37R/MILES DRIVEN/AVE. WEEKENO/C

$0-10 \quad 11-20 \quad 21-50$ OVER 50 DK/NO AN

SWER

TOTAL

STANDARD FED. ADMNSTRATV REGIONS

$\begin{array}{llllllr}\text { I/NEW ENGLNO STS } & 24.07 & 16.67 & 22.22 & 31.48 & 5.56 & 54 \\ \text { IIINW YRK/NW JRY } & 33.04 & 17.86 & 22.32 & 24.11 & 2.68 & 112 \\ \text { III/MIO ATLANTIC } & 32.38 & 14.29 & 21.90 & 27.62 & 3.81 & 105 \\ \text { IV/SOUTH EAST } & 30.46 & 15.09 & 25.86 & 24.71 & 2.87 & 174 \\ \text { V/GREAT LAKES } & 25.12 & 12.81 & 34.48 & 23.15 & 4.43 & 203 \\ \text { VI/MID SCUTH } & 25.00 & 10.71 & 27.68 & 33.93 & 2.68 & 112 \\ \text { VII/PLAINS } & 26.42 & 13.87 & 30.19 & 22.64 & 1.89 & 53 \\ \text { VIII/NW PLAINS } & 46.88 & 6.25 & 37.50 & 6.25 & 3.13 & 32 \\ \text { IX/WEST COAST } & 25.62 & 11.57 & 26.79 & 32.23 & 5.79 & 121 \\ \text { X/NORTH WEST } & 20.59 & 17.65 & 20.59 & 35.29 & 5.88 & 34\end{array}$

RESPONDENT'S AGE

$\begin{array}{lcccccc}18-24 & 19.40 & 18.66 & 31.34 & 29.10 & 1.49 & 134 \\ 25-44 & 23.16 & 12.42 & 30.74 & 30.53 & 3.16 & 475 \\ 45 \text { AND OVER } & 37.60 & 14.83 & 21.23 & 20.97 & 5.37 & 391 \\ \text { RESPONDENTIS EDUCATION } & & & & & & \\ \text { LTHIGH SCHOOL } & 46.43 & 14.29 & 25.89 & 9.82 & 3.57 & 112 \\ \text { HIGH SCHOOL GRAD } & 26.95 & 15.36 & 27.49 & 26.68 & 3.50 & 371 \\ \text { SOME COLLEGE } & 25.93 & 12.96 & 26.67 & 28.89 & 5.56 & 270 \\ \text { COLLEGE GRAD } & 24.70 & 13.77 & 27.53 & 31.58 & 2.43 & 247\end{array}$

RESPONDENT'S SEX

$\begin{array}{lrrrrrr}\text { MALE } & 16.03 & 15.43 & 29.66 & 36.47 & 3.41 & 499 \\ \text { FEMALE/HOME } & 49.79 & 10.29 & 22.63 & 13.58 & 3.70 & 243 \\ \text { FEMALE/EMPLOYEO } & 31.78 & 15.50 & 28.29 & 19.77 & 4.65 & 258 \\ \text { PRIMARY SOURCE OF FAMILY INCOME/C } & & & & \\ \text { SLF-EMPL/WHITE C } & 22.56 & 13.72 & 28.37 & 32.09 & 3.26 & 430 \\ \text { BLUE-COLLAR } & 24.28 & 13.17 & 30.86 & 29.22 & 2.47 & 243 \\ \text { NOT EMPLOYED } & 46.48 & 14.55 & 19.72 & 13.62 & 5.63 & 213 \\ \text { OTHER } & 24.56 & 17.54 & 28.07 & 24.56 & 5.26 & .114 \\ \text { AGGREGATE } & 28.30 & 14.20 & 27.10 & 26.60 & 3.80 & 1000\end{array}$


TABLE 16 (continued)

TARRANCE \& ASSC. - SAFETY BELT (MOOO) - \#2017

P37R/MILES DRIVEN/AVE. WEEKEND/C

$0-10 \quad 11-20 \quad 21-50$ OVER 50 DK/NO AN

SWER TOTAL

USER CATAGORIES

NOT APDLICABLE

FREQ USEOS/CNFRM

SMTM USER/LIKELY

$27.97 \quad 14.47$

$24.7 \dot{6}$

$25.97 \quad 12.12$

31.60

$28.26 \cdot 1.3 .30$

29.35

$29.17 \quad 17.26$

25.60

INFREQ USR/LIKLY

$29.14 \quad 11.26$

20.49

27.97
25.97
22.83
24.40
30.46

$4.8 \dot{2}$

4.33

$31 i$

231

92

168

3.57

2.65

151

PE5/WHERE DO YOU LIVE

IN $A$ SIG CITY

SUBURBSIBIG CITY

IN A SIAALL CITY

TOWIVIVLLAGE

IN THE COUNTRY

DK/NO ANSWER
29.63

24.04

32.59

34.12

21.26

0.00
13.23

15.68

13.84

14.71

12.60

0.00
23.93

30.31

27.23

17.06

33.86

60.67
4.23

4.18

4.02

2.94

2.36
$0.00 \quad 33.33$

189

287

224

170

127

FREQ-DHV-1 HE ORAKING

DAILY TO I/MONTH
SEVERAL TMS/YR
NEVER

18.00

24.87

16.50

32.00

16.58

32.78

1.2 .69

.27 .46

25.37

$31 \cdot 50$
$28 \cdot 50$

2.00

200

2.59

4.78 .

193

607

AGGREGATE

$28.30 \quad 14.20$

27.10

26.60

3.80

1000 
61

TABLE 17 - NUMBER OF TRIPS OVER 200 MILES/PAST YEAR

TARRANCE \& ASSC. - SAFETY BELT (MOOD) - \#2017

P38R-TRIPS OVER 200 MLS/LAST YR

$0-1 \quad 2-45 \&$ OVER DK/NO AN

SWER TOTAL

STANOARD FED. ADMNSTRATV REGIONS

$\begin{array}{lllllr}\text { I/NEW ENGLND STS } & 33.33 & 37.04 & 29.63 & 0.00 & 54 \\ \text { IIINW YRK/NW JRY } & 44.64 & 33.04 & 22.32 & 0.00 & 112 \\ \text { III/MID ATLANTIC } & 39.05 & 33.10 & 22.86 & 0.00 & 105 \\ \text { IV/SOUTH EAST } & 44.25 & 29.31 & 24.14 & 2.30 & 174 \\ \text { V/GREAT LAKES } & 35.47 & 34.98 & 28.08 & 1.48 & 203 \\ \text { VI/MID SOUTH } & 34.82 & 25.89 & 36.61 & 2.68 & 112 \\ \text { VII/PLAINS } & 33.96 & 35.85 & 30.19 & 0.00 & 53 \\ \text { VIII/NW PLAINS } & 43.75 & 43.75 & 12.50 & 0.00 & 32 \\ \text { IX/WEST COAST } & 42.15 & 30.58 & 24.79 & 2.48 & 121 \\ \text { X/NORTH WEST } & 17.65 & 35.29 & 47.06 & 0.00 & 34\end{array}$

RESPONOENT'S AGE

$\begin{array}{llllll}18-24 & 32.84 & 37.31 & 29.10 & 0.75 & 134 \\ 25-44 & 36.63 & 33.26 & 29.26 & 0.84 & 475 \\ 45 \text { AND OVER } & 42.97 & 31.20 & 23.79 & 2.05 & 391\end{array}$

RESPONOENTIS EDUCATION

$\begin{array}{llllll}\text { LT HIGH SCHOOL } & 60.71 & 19.75 & 17.86 & 2.68 & 112 \\ \text { HIGH SCHDOL GRAD } & 42.05 & 33.69 & 22.91 & 1.35 & 371 \\ \text { SOME COLLEGE } & 34.81 & 35.93 & 28.52 & 0.74 & 270 \\ \text { COLLEGE GRAD } & 27.53 & 35.22 & 36.03 & 1.21 & 247\end{array}$

RESPONDENT IS SEX

$\begin{array}{llllll}\text { MALE } & 30.66 & 33.07 & 34.47 & 1.80 & 499 \\ \text { FEMALE/HOME } & 51.03 & 30.86 & 16.87 & 1.23 & 243 \\ \text { FEMALE/EMPLOYED } & 42.25 & 34.88 & 22.48 & 0.39 & 258\end{array}$

PRIMARY SOURCE OF FAMILY INCOME/C

\begin{tabular}{|c|c|c|c|c|c|}
\hline $\begin{array}{l}\text { SLF-EMPL/WHITE C } \\
\text { BLUE-COLLAR } \\
\text { NOT EMPLOYED } \\
\text { OTHER }\end{array}$ & $\begin{array}{l}32.79 \\
39.09 \\
52.11 \\
34.21\end{array}$ & $\begin{array}{l}31.86 \\
37.04 \\
25.64 \\
36.84\end{array}$ & $\begin{array}{l}34.42 \\
22.63 \\
17.37 \\
27.19\end{array}$ & $\begin{array}{l}0.93 \\
1.23 \\
1.88 \\
1.75\end{array}$ & $\begin{array}{l}430 \\
243 \\
213 \\
114\end{array}$ \\
\hline$\triangle G G R E G A T E$ & 38.60 & 33.00 & 27.1 .0 & 1.30 & 1000 \\
\hline
\end{tabular}


TABLE 17 (continued)

62

TAROANCE \& ASSC. - SAFETY BELT (MOOD) - \#2017

$$
\begin{aligned}
& \text { P38R-TRIPS OVER } 200 \text { MLS/LAST YR } \\
& \begin{array}{rr}
0-1 & 2-45 \& \text { OVER DK/NO AN } \\
\text { SWER }
\end{array}
\end{aligned}
$$

TOTAL

USER CATAGORIES

NOT APPLICABLE

FREQ USERS/CNFRM

SMTM USEQ/LIKELY

SMTM USER, UNLKLY

INFREQ USR/LIKLY

$39.5 \dot{5}$

30.74

32.61

47.02

39.07
$31.5 i$

37.66

34.78

29.17

33.11

27.97
30.74
31.52
22.112
25.17

0.96
0.87
1.09
1.79
2.65

$31 i$

231

92

168

151

PS5/WHERE DO YOU LIVE

IV A EIG CITY

SUSURBS/BIG CITY

IN A SMALL CITY

TOWN/VILLAGE

IN THE COUNTRY

DK/NO $\triangle N S$ SER
35.98

37.28

40.63

42.35

36.22

66.67
29.63

33.45

34.82

32.35

35.43

0.00
32.28

28.22

23.21

24.71

20.77

33.33
2.12

1.05

1.34

0.59

1.57

0.00
189

287

224

170

127

$\because 3$

FREQ-DRV-I HP. DRNKNG

DAILY TO I/MONTH SEVERAL TMS/YR NIEVER.

AGGREGATE

$\begin{array}{lllll}34.00 & 30.50 & 34.00 & 1.50 & 200 \\ 31.61 & 34.20 & 32.64 & 1.55 & 193 \\ 42.34 & 33.44 & 23.06 & 1.15 & 607 \\ 38.60 & 33.00 & 27.10 & 1.30 & 1000\end{array}$


63

TABLE 18 - CHANGE IN NUMBER OF TRIPS OVER 200 MILES

TARRANCE \& ASSC. - SAFETY BELT (MOOD) - \#2017

P39/IS THIS MORE OR LESS THAN YEAR BEFO

MORE UNSURE NO DIFFE LESS

RENCE

TOTAL

STANDARO FED. ADMNSTRATV REGIONS

$\begin{array}{lllllr}\text { I/NEW ENGLNO STS } & 22.22 & 7.41 & 38.89 & 31.48 & 54 \\ \text { II/NW YRK/NW JRY } & 14.29 & 0.89 & 56.25 & 28.57 & 112 \\ \text { III/MID ATLANTIC } & 15.24 & 0.95 & 48.57 & 35.24 & 105 \\ \text { IV/SOUTH EAST } & 16.09 & 4.02 & 41.38 & 38.51 & 174 \\ \text { V/GREAT LAKES } & 16.75 & 0.99 & 52.71 & 29.56 & 203 \\ \text { VI/MIO SOUTH } & 23.21 & 2.68 & 44.64 & 29.46 & 112 \\ \text { VII/PLAINS } & 30.19 & 3.77 & 35.85 & 30.19 & 53 \\ \text { VIII/NW PLAINS } & 21.88 & 0.00 & 43.75 & 34.38 & 32 \\ \text { IX/WEST COAST } & 18.18 & 1.65 & 37.19 & 42.98 & 121 \\ \text { X/NORTH WEST } & 26.47 & 2.94 & 26.47 & 44.12 & 34\end{array}$

RESPONOENT'S AGE

$\begin{array}{llllll}18-24 & 31.34 & 2.24 & 26.12 & 40.30 & 134 \\ 25-44 & 20.00 & 1.05 & 43.16 & 35.79 & 475 \\ 45 . \text { AND OVER } & 12.53 & 3.84 & 53.96 & 29.67 & 391\end{array}$

RESPONOENT'S EDUCATION

$\begin{array}{llllll}\text { LT HIGH SCHOOL } & 15.18 & 3.57 & 48.21 & 33.04 & 112 \\ \text { HIGH SCHOOL GRAD } & 19.41 & 1.89 & 46.09 & 32.61 & 371 \\ \text { SOME COLLEGE } & 21.48 & 2.22 & 42.22 & 34.07 & 270 \\ \text { COLLEGE GRAD } & 15.79 & 2.43 & 45.34 & 36.44 & 247\end{array}$

RESPONDENT'S SEX

$\begin{array}{llllll}\text { MALE } & 18.04 & 2.00 & 44.29 & 35.67 & 499 \\ \text { FEMALE/HOME } & 17.28 & 3.70 & 47.74 & 31.28 & 243 \\ \text { FEMALE/EMPLOYED } & 20.93 & 1.05 & 44.19 & 33.33 & 258\end{array}$

PRIMARY SOURCE OF FAMILY INCOME/C

\begin{tabular}{|c|c|c|c|c|}
\hline $\begin{array}{l}\text { SLF-EMPL/WHITE C } \\
\text { BLUE-COLLAR } \\
\text { NOT EMPLOYED } \\
\text { OTHER }\end{array}$ & $\begin{array}{l}18.84 \\
22.22 \\
14.08 \\
18.42\end{array}$ & $\begin{array}{l}1.63 \\
1.65 \\
4.69 \\
1.75\end{array}$ & $\begin{array}{l}48.14 \\
39.09 \\
48.36 \\
40.35\end{array}$ & $\begin{array}{l}31.40 \\
37.04 \\
32.86 \\
39.47\end{array}$ \\
\hline AGGREGATE & 18.60 & 2.30 & 45.10 & 34.00 \\
\hline
\end{tabular}


TABLE 18 (continued)

64

TARPANCE \& ASSC. - SAFETY BELT (MOOD) - \#2017

P39/IS THIS MORE OR LESS THAN YEAR BEFO

MORE UNSURE NO DIFFE LESS

RENCE

TOTAL

USER CATAGORIES

NOT $\triangle P P L I C A B L E$

FREO USERS/CNFRM

SMTM USER/LIKELY

SMTM USER/UNLKLY

INFREQ USR/LIKLY

21.54

18.61

17.39

17.26

INFREO USR/CNFRM

19.21

1.93
3.46
1.09
1.79
3.31

$42.4 \dot{4}$

34.08

42.86

35.06

42.39

39.13

50.00

30.95

$40.36 \quad 31.13$

$31 i$

231

92.

168

151

PSS/WHEPE DO YOU LIVE

\begin{tabular}{|c|c|c|c|c|c|}
\hline $\begin{array}{l}\text { IN A BIG CITY } \\
\text { SUBURBS/BIG CITY } \\
\text { IN A SIALLL CITY } \\
\text { TONAN IVILLCGE } \\
\text { IN THE COUNTRY } \\
\text { OK/NO ANSWER }\end{array}$ & $\begin{array}{l}23.81 \\
18.82 \\
19.64 \\
15.88 \\
12.60 \\
0.00\end{array}$ & $\begin{array}{l}2.12 \\
3.14 \\
1.77 \\
1.76 \\
1.57 \\
33.33\end{array}$ & $\begin{array}{l}38.62 \\
45.99 \\
44.20 \\
50.59 \\
47.24 \\
33.33\end{array}$ & $\begin{array}{l}35.45 \\
32.06 \\
34.38 \\
31.76 \\
38.58 \\
33.33\end{array}$ & $\begin{array}{r}189 \\
287 \\
224 \\
170 \\
127 \\
3\end{array}$ \\
\hline FREQ-OKV-! HR DFN & & & & & \\
\hline $\begin{array}{l}\text { CAILY TO I/MONTH } \\
\text { SEVEPALL TMS/YR } \\
\text { NEVER }\end{array}$ & $\begin{array}{l}19.50 \\
20.73 \\
17.63\end{array}$ & $\begin{array}{l}1.50 \\
0.52 \\
3.13\end{array}$ & $\begin{array}{l}40.50 \\
48.70 \\
45.47\end{array}$ & $\begin{array}{l}38.50 \\
30.05 \\
33.77\end{array}$ & $\begin{array}{l}200 \\
193 \\
607\end{array}$ \\
\hline$\triangle G G R E G \triangle T E \cdots$ & 18.60 & 2.30 & 45.10 & 34.00 & 1000 \\
\hline
\end{tabular}


TABLE 19 - YEAR OF LAST OVER 200-MILE TRIP

TARRANCE \$ ASSC. - SAFETY BELT (MOOD) - 2017

PLCR/YEAR OF LAST 200 MILE TRIPIC

NONE BEFORE $1 \quad 1980 \quad 198$

1983 DK/NO AN

SWER TOTAL

STANCARC FED. ACMNSTRATV REUIONS

i/AJE* ENGLNC STS

!I/NW YRK/PWW JRY

II!/MID ATLANTIC

IV/SOUTH EAST

V/TPEAT LAKES

$V ! / 4 ! O$ SOUTH

$\checkmark:$ :OLJINS

VITIINN PLAINS

IX/NEST COAST

$X / N O Q T A$ WEST

DESPONDEAT'S AGE

$1+2-24$

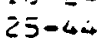

-5 AITO CVER

3.73

2.11

3.32

\subsection{4}

3.53

10.74

ZESPONDENT IS EDUCAT:ON

IT +15- SCHUOL

IOM SCHOOL Bर̃OO

SOME COLLEGE

COLLEGE GRAD

$\begin{array}{ll}4.46 & 14.29 \\ 4.06 & 7.28 \\ 2.22 & 5.55 \\ 0.81 & 7.29\end{array}$

8.93

4.04

1.48

1.21

8.93

9.43

5.56

5.67

34.32

47.44

52.22

47.37

20.54

24.80

28.89

35.22

6.21

8.23

$\begin{array}{lr}2.40 & 5.41 \\ 4.53 & 11.93 \\ 1.96 & 7.75\end{array}$

3.41

3.70
2.33

8.91

46.29

45.68
50.78

33.47

20.58

24.42

$2.8 !$

5.35

3.88

243

258

PQIMARY SOURCE OF FAMILY INCOME/C.

SLP -EMPL/WHITE C
BLUE -COLLAR
MOT EMPLOYEO
OTHEE
HOSPEOTHE

AGTEGATE

\subsection{6}

3.70

3.75

6.05

7.82

9.86

46.05

52.25

40.38

54.39

33.95

25.10

19.72

27.19

47.30

28.00

7.40
3.02

2.06

7.51

430

243

$2 ! 3$

3.70

1000 
TABLE 19 (continued)

TAPAANCE S SSSC. - SAFETY EE:T (MOOD) - 2017

PUIR/YEAR OF :AST 200 MILE TRIP/C

AONE BEFORE 1 GEC $\quad$ I980 LQEI $1982 \quad 1983$ OK/NO AN SWER TOTAL

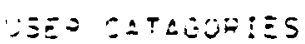

オ广 \&

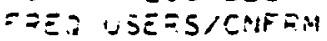

SATU UIEZ/LI-ELY

EYTU JSED/LiNLXLY

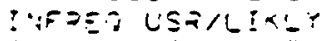

$$
\begin{array}{ll}
2.39 & 7.48 \\
3.46 & 7.36 \\
1.04 & 4.35 \\
3.57 & 1 ! .31 \\
1.99 & 7.23
\end{array}
$$

$$
\begin{aligned}
& 3.22 \\
& 1.30 \\
& 2.17 \\
& 4.17 \\
& 4.54
\end{aligned}
$$

7.72

4.76

11.76

7.14

6.52

$\begin{array}{rr}48.23 & 26.69 \\ 51.52 & 29.87 \\ 52.17 & 25.00 \\ 42.25 & .29 .57 \\ 44.37 & .27 .31\end{array}$

3.86

1.73

3.26

2.98

m........

DSE/HAEE DO YOU LiVF

$\because \pm \div \amalg \sigma 0:^{-v}$

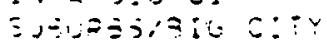

$\because \because 5+1-2$ E $i: Y$

T?:I/VILLAOE

iU - TE CEUNTJ4

ZR/NG SASmEF

$$
\begin{array}{lr}
3.17 & 5.32 \\
3.1 .0 & 7.57 \\
5.25 & 7.14 \\
5.29 & 3.24 \\
1.57 & 9.45 \\
0.00 & 33.33
\end{array}
$$

2.12
2.79

3.37

4.12

3.94

0.00

$\begin{array}{rr}10.05 & 47.09 \\ 5.92 & 48.08 \\ 5.70 & 52.23 \\ 7.65 & 43.53 \\ 7.07 & 42.52 \\ 0.00 & 33.33\end{array}$

29.57
29.62
26.36
27.55
27.56

3.17

2.79

3.13

3.53

7.09

33.33

$31 i$
231
92
$1 \leq 3$
151

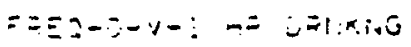

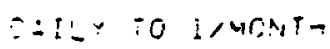

$\begin{array}{ll}0.50 & 5.50 \\ 0.52 & 5.22 \\ 4.25 & \$ .73\end{array}$

\section{50}

3.11

5.50

50.50

0.00

3.13

3.29
7.74

32.00

2.50

45.08

35.75

1.04

193

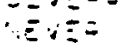

2. 20

7.50

3.20

7.40

47.30

28.00 .

3.70

159

23i

224

170

$1 \leq 7$

3

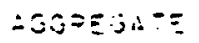

(2)

7.40


TABLE 20 - SEASON OF LAST 200 MILE TRIP

TARRANCE \& ASSC. - SAFETY BELT (MOOD) - $\# 2017$

P4ORI/SEASON/LAST 200 MILE TRIP/C

NONE SPRIVG/S FALL/WIN DK/NO AN

UMMER TER SWER

TOTAL

STANDARD FED. ADMNSTRATV REGIONS

I/NEW ENGLND STS

II/NW YRK/NW JRY

III/MIO ATLANTIC

IV/SOUTH EAST

V/GREAT LAKES

VI/MIO SOUTH

VII/PLAINS

VIII/NW PLAINS

IX/WEST COAST

$X / N O R T H$ WEST

$\begin{array}{lll}5.56 & 33.33 & 59.26 \\ 4.46 & 33.04 & 52.68 \\ 2.86 & 35.24 & 52.38 \\ 4.02 & 32.76 & 55.17 \\ 3.45 & 42.36 & 49.75 \\ 2.68 & 27.68 & 61.61 \\ 1.89 & 37.74 & 58.49 \\ 0.00 & 59.38 & 37.50 \\ 2.48 & 38.02 & 54.55 \\ 0.00 & 32.35 & 67.65\end{array}$

1.85

9.82

9.52

8.05

4.43

8.04

1.89

3.13

4.96

0.00

54

112

105

174

203

112

53

32

121

34

RESPONDENT'S AGE

\begin{tabular}{|c|c|c|c|c|}
\hline $\begin{array}{l}18-24 \\
25-44 \\
45 \text { AND OVER }\end{array}$ & $\begin{array}{l}4.48 \\
2.11 \\
4.09\end{array}$ & $\begin{array}{l}37.31 \\
38.74 \\
32.74\end{array}$ & $\begin{array}{l}55.22 \\
54.53 \\
53.96\end{array}$ & $\begin{array}{l}2.99 \\
4.63 \\
9.21\end{array}$ \\
\hline \multicolumn{5}{|c|}{ RESPONOENT'S EDUCATION } \\
\hline $\begin{array}{l}\text { LT HIGH SCHOOL } \\
\text { HIGH SCHOCL GRAO } \\
\text { SOME COLLEGE } \\
\text { COLLEGE GRAD }\end{array}$ & $\begin{array}{l}6.25 \\
4.58 \\
2.22 \\
0.81\end{array}$ & $\begin{array}{l}36.61 \\
38.81 \\
38.89 \\
29.15\end{array}$ & $\begin{array}{l}42.86 \\
51.48 \\
53.70 \\
64.78\end{array}$ & $\begin{array}{r}14.29 \\
5.12 \\
5.19 \\
5.26\end{array}$ \\
\hline
\end{tabular}

RESPONDENT'S SEX

$\begin{array}{llllll}\text { MALE } & 3.01 & 32.46 & 59.12 & 5.41 & 499 \\ \text { FEMALE/HOME } & 4.94 & 39.92 & 47.74 & 7.41 & 243 \\ \text { FEMALE/EMPLOYED } & 1.94 & 39.92 & 51.55 & 6.59 & 258\end{array}$

PRIMARY SOURCE OF FAMILY INCOME/C

$\begin{array}{lrrrrr}\text { SLF-EMPL/WHITE C } & 2.09 & 34.19 & 57.91 & 5.81 & 430 \\ \text { BLUE-COLLAR } & 4.12 & 42.80 & 49.79 & 3.29 & 243 \\ \text { NOT EMPLOYED } & 5.16 & 31.46 & 52.11 & 11.27 & 213 \\ \text { OTHER } & 1.75 & -38.60 & 55.26 & 4.39 & 114 \\ \text { AGGREGATE } & 3.20 & 36.20 & 54.40 & 6.20 & 1000\end{array}$


TABLE 20 (continued)

TARPANCE \& ASSC. - SAFETY EELT (MOOD) - \#2017

$$
\begin{aligned}
& \text { P4ORI/SEASON/LAST } 200 \text { MILE TRIP/C } \\
& \text { NONE SPRIVG/S FALL/WIN DK/NO AN } \\
& \text { UMMEF TOR TOTAL }
\end{aligned}
$$

USER CATAGURIES

NOT $\triangle P P L I C A B L E$

FREQ USERS/CNFRM

SMTM USER/LIKELY

SMTM USER/UNLKLY

INFREQ USR/LIKLY

INFREQ USR/CNFRM

$\begin{array}{rrrr}3.86 & 35.69 & 54.66 & 5.79 \\ 3.46 & 32.90 & 58.87 & 4.76 \\ 2.17 & \cdots .45 .65 & 48.91 & 3.26 \\ 3.57 & 39.29 & 51.79 & 5.36 \\ 1.99 & 32.45 & 53.64 & 11.92\end{array}$

311

231

92

168

151

PS5/WHERE DO YOU LIVE

IN $A B I G$ CITY

SUBURBS/BIG CITY

IN A SMALL CITY

TOW'N/VILLAGE

IN THE COUNTEY

DK/NO DNSNER

$\begin{array}{ll}4.23 & 35.45 \\ 3.48 & 35.19 \\ 0.89 & 34.82 \\ 5.29 & 37.65 \\ 1.57 & 40.16 \\ 33.33 & 33.33\end{array}$

55.03

56.45

57.59

51.76

48.03

0.00
139

287

224

170

127

3

FREQ-DHV-1 HR DRNKNG

DAILY TO I/MONTH
SEVERAL TMS/YR
NEVER.

AGGREGATE

$\begin{array}{lll}0.50 & 33.00 & 63.00 \\ 0.52 & 38.34 & 50.03 \\ 4.94 & 35.57 & 50.41\end{array}$

3.50

3.11

8.07

200

193

607

$3.20 \quad 35.20 \quad 54.40$

6.20

1000 
TABLE 21 - NUMBER OF PERSONS IN CAR/LAST TRIP

TARRANCE \& ASSC. - SAFETY BELT (MOOD) - \#2017

P4IR/NUMBER OF PPL IN CAR/LAST TRIP/C

ONE TWO THREE FOUR OR DK/NO AN
MORE

STANDARO FED. AOMNSTRATV REGIONS

$\begin{array}{lrrrrrr}\text { I/NEW ENGLND STS } & 20.37 & 27.78 & 20.37 & 29.63 & 1.85 & 54 \\ \text { II/NW YRK/NW JRY } & 11.61 & 36.61 & 9.82 & 35.71 & 6.25 & 112 \\ \text { III/MID ATLANTIC } & 18.10 & 33.33 & 16.19 & 26.67 & 5.71 & 105 \\ \text { IV/SOUTH EAST } & 13.22 & 33.33 & 12.64 & 37.36 & 3.45 & 174 \\ \text { V/GREAT LAKES } & 12.32 & 34.98 & 18.72 & 33.00 & 0.99 & 203 \\ \text { VI/MID SOUTH } & 14.29 & 25.89 & 20.54 & 36.61 & 2.68 & 112 \\ \text { VII/PLAINS } & 7.55 & 35.85 & 16.98 & 37.74 & 1.89 & 53 \\ \text { VIII/NW PLAINS } & 6.25 & 25.00 & 25.00 & 40.63 & 3.13 & 32 \\ \text { IX/WEST COAST } & 9.92 & 38.02 & 12.40 & 36.36 & 3.31 & 121 \\ \text { XINORTH WEST } & 8.82 & 44.12 & 11.76 & 35.29 & 0.00 & 34\end{array}$

RESPONOENT'S AGE

$\begin{array}{lrrrrrr}18-24 & 16.42 & 25.37 & 14.18 & 41.79 & 2.24 & 134 \\ 25-44 & 13.89 & 25.89 & 15.79 & 42.32 & 2.11 & 475 \\ 45 \text { AND OVER } & 10.23 & 46.04 & 16.37 & 22.76 & 4.60 & 391 \\ \text { RESPONDENTIS EOUCATION } & & & & & & \\ \text { LTHIGH SCHOOL } & 9.82 & 30.36 & 16.07 & 37.50 & 6.25 & 112 \\ \text { HIGH SCHOOL GRAD } & 9.43 & 32.61 & 18.06 & 37.47 & 2.43 & 371 \\ \text { SONE COLLEGE } & 17.04 & 32.22 & 1.4 .81 & 32.59 & 3.33 & 270 \\ \text { COLLEGE GRAD } & 14.57 & .38 .46 & 13.36 & 31.17 & 2.43 & 247\end{array}$

RESPONDENTIS SEX

$\begin{array}{lrrrrrr}\text { MALE } & 17.64 & 33.67 & 16.23 & 30.66 & 1.80 & 499 \\ \text { FEMALE/HOME } & 9.05 & 35.39 & 14.81 & 36.21 & 4.53 & 243 \\ \text { FEMALE/EMPLOYED } & 6.98 & 32.17 & 15.89 & 40.70 & 4.26 & 258 \\ \text { PRIMARY SOURCE OF FAMILY INCOME/C } & & & & \\ & & & & & & \\ \text { SLF-EMPL/WHITE C } & 17.21 & 31.86 & 16.05 & 32.09 & 2.79 & 430 \\ \text { BLUE-COLLAR } & 7.82 & 28.81 & 19.75 & 41.98 & 1.65 & 243 \\ \text { NOT EMPLOYED } & 10.80 & 42.25 & 12.21 & 28.64 & 6.10 & 213 \\ \text { OTHER } & 10.53 & 35.09 & 13.16 & 39.47 & 1.75 & 114 \\ \text { AGGREGATE } & 12.80 & 33.70 & 15.80 & 34.60 & 3.10 & 1000\end{array}$


TABLE 21 (continued)

TARRANCE \& ASSC. - SAFETY BELT (MOOD) - \#2017

P4IR/NUMBER OF PPL IN CAR/LAST TRIP/C

ONE TWO THREE FOUR OR DK/NO AN MORE SWER

TOTAL

USER CATAGORIES

NOT APPLICABLE

FREO USERS/CNFRM

SMTM USER/LIKELY

SMTM USER/UNULLLY

INFREO USR/LIKLY

INFREQ USR/CNFRM

$\begin{array}{rlllll}13.83 & 35.37 & 13.83 & 33.76 & 3.22 \\ 12.55 & 33.33 & 15.15 & 37.66 & 1.30 \\ 7.61 & 39.04 & 15.22 & 38.04 & 1.09 \\ 12.50 & 32.14 & 22.02 & 30.36 & 2.98 \\ 15.23 & 30.46 & 16.56 & 30.46 & 7.28\end{array}$

$31 i$

231

92

168

151

PS5/WHERE OO YOU LIVE

IN A BIG CITY

SUBURES/BIG CITY

TOWN/VILLAGE

IV THE COUNTRY

OKINO ANSWER
IN A SMALL CITY

$\begin{array}{rr}14.81 & 34.39 \\ 16.33 & 35.54 \\ 9.82 & 34.82 \\ 12.94 & 32.35 \\ 7.09 & 29.35 \\ 0.00 & 33.33\end{array}$

17.05

14.29

15.18

11.18

22.05

0.00
3.17

2.44

2.23

2.35

6.30

33.33
189

287

224

170

127

3

FREQ-DQV-1 HP DRNKNG

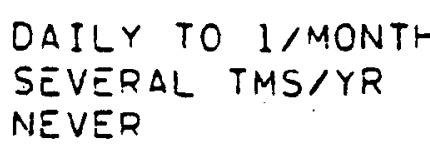

$\triangle G G R E G A T E$

21.00

10.36

10.87

33.50

38.34

32.29

16.00

17.10

15.32

28.00

33.68

37.07

15.80

34.60

33.70

12.80

12.80 
TABLE 22 - OROVE MORE OR LESS THAN 1982

TARRANCE \& ASSC. - SAFETY BELT (MOOD) - \#2017

P42/FAMILY DROVE MORE/LESS THAN IN 1981

more less the same unsure total

STANDARO FED. ADMNSTRATV REGIONS

$\begin{array}{lllllr}\text { I/NEW ENGLND STS } & 27.78 & 37.04 & 33.33 & 1.85 & 54 \\ \text { II/NW YRK/NW JRY } & 33.93 & 30.36 & 32.14 & 3.57 & 112 \\ \text { III/MID ATLANTIC } & 31.43 & 31.43 & 34.29 & 2.86 & 105 \\ \text { IV/SOUTH EAST } & 31.03 & 35.21 & 31.03 & 1.72 & 174 \\ \text { V/GREATLLKES } & 28.57 & 36.95 & 33.99 & 0.49 & 203 \\ \text { VI/MID SOUTH } & 38.39 & 35.71 & 25.00 & 0.89 & 112 \\ \text { VIIIPLAINS } & 37.74 & 33.96 & 28.30 & 0.00 & 53 \\ \text { VIII/NW PLAINS } & 21.88 & 50.00 & 25.00 & 3.13 & 32 \\ \text { IX/WEST COAST } & 34.71 & 36.36 & 26.45 & 2.48 & 121 \\ \text { X/NOPTH WEST } & 44.12 & 41.18 & 14.71 & 0.00 & 34\end{array}$

RESPONOENT'S AGE

$\begin{array}{llllll}18-24 & 52.99 & 26.87 & 18.66 & 1.49 & 134 \\ 25-44 & 35.79 & 37.89 & 25.47 & 0.84 & 475 \\ 45 \text { AND OVER } & 21.48 & 36.06 & 39.64 & 2.81 & 391\end{array}$

RESPONOENT'S EDUCATION

$\begin{array}{llllll}\text { LT HIGH SCHOOL } & 22.32 & 43.75 & 30.36 & 3.57 & 112 \\ \text { HIGH SCHOOL GRAD } & 36.93 & 32.88 & 28.30 & 1.89 & 371 \\ \text { SOME COLLEGE } & 33.33 & 36.67 & 28.52 & 1.48 & 270 \\ \text { COLLEGE GRAD } & 29.55 & 35.22 & 34.41 \ldots & 0.81 & 247\end{array}$

RESPONOENT 'S SEX

$\begin{array}{llllll}\text { MALE } & 32.06 & 34.07 & 31.86 & 2.00 & 499 \\ \text { FEMALE/HOME } & 28.81 & 38.27 & 31.28 & 1.65 & 243 \\ \text { FEMALE/EMPLOYED } & 36.82 & 36.43 & 25.58 & 1.16 & 258\end{array}$

PRIMARY SOURCE OF FAMILY INCOME/C

$\begin{array}{llllll}\text { SLF-EMPL/WHITE C } & 33.26 & 31.40 & 34.65 & 0.70 & 430 \\ \text { BLUE-COLLAR } & 34.98 & 40.33 & 23.87 & 0.82 & 243 \\ \text { NOT EMPLOYED } & 24.88 & 41.31 & 29.58 & 4.23 & 213 \\ \text { OTHER . } & 38.60 & 31.58 & 27.19 & 2.63 & 114 \\ \text { AGGREGATE } & 32.50 & 35.70 & 30.10 & 1.70 & 1000\end{array}$


TABLE. 22 (continued)

TARRANCE \& ASSC. - SAFETY BELT (MOOD) - \#2017

P42/FAMILY OROVE MORE/LESS THAN IN 1981

MORE LESS THE SAME UNSURE TOTAL

USER CATAGORIES

NOT $\triangle P D L I C A B L E$

FREG USERS/CNFRM

SMTM USER/LIKELY

SMTM USER/UNLKLY

INFREO LISR/LIKLY

INFREQ USR/CNFRM

$\begin{array}{ll}34.41 & 34.08 \\ 33.77 & 38.5\end{array}$

$34.78 \quad 33.70$

$29.17 \quad 35.71$

$31.13 \quad 35.76$ $29.90^{\circ}$

26.84

30.43

34.52

24.80
$1.6 \dot{1}$

0.87

1.09

0.60

3.31
$31 \dot{1}$

231

92

168

151

P65/WHERE DO YOU LIVE

IN A BIG CITY

SUBUROS/BIG CITY

IV A SMALL CITY

TOVIN/VILLAGE

IY THE COUNTRY

DK/NO LNSWER

FREO-DPV-1 HF DRNKNG

OAILY TO I/MONTH

SEVEPAL TMS/YR

NEVER

AGGREGATE

$\begin{array}{rr}37.04 & 31.75 \\ 29.97 & 35.59 \\ 31.25 & 40.63 \\ 34.012 & 35.29 \\ 32.28 & 30.71 \\ 0.00 & 66.67\end{array}$

$$
\begin{array}{r}
28.04 \\
31.71 \\
27.68 \\
29.41 \\
35.43 \\
0.00
\end{array}
$$

3.17

1.74

0.45

1.18

1.57

33.33
189

287

224

170

127

3

AGGREGATE

$\begin{array}{rrrrr}33.00 & 34.50 & 31.00 & 1.50 & 200 \\ 33.68 & 33.68 & 32.64 & 0.00 & 193 \\ 31.96 & 36.74 & 29.00 & 2.31 & 607 \\ 32.50 & 35.70 & 30.10 & 1.70 & 1000\end{array}$


TABLE 23 - CHANGED DRIVING HABITS IN PAST YEAR TARRANCE \& ASSC. - SAFETY BELT (MOOD) - \$2017

P44/DRIVING HABITS CHANGED IN THE LAST
YES UNSURE TOTAL

STANDARD FED. AOMNSTRATV REGIONS

$\begin{array}{lllr}\text { I/NEW ENGLND STS } & 22.22 & 77.78 & 54 \\ \text { II/NW YRK/NW JRY } & 39.29 & 60.71 & 112 \\ \text { III/MID ATLANTIC } & 26.67 & 73.33 & 105 \\ \text { IV/SOUTH EAST } & 36.21 & 63.79 & 174 \\ \text { V/GREAT LAKES } & 26.11 & 73.89 & 203 \\ \text { VI/MIO SOUTH } & 34.82 & 65.18 & 112 \\ \text { VII/PLIINS } & 28.30 & 71.70 & 53 \\ \text { VIII/NH PLAINS } & 25.00 & 75.00 & 32 \\ \text { IX/WEST COAST } & 25.62 & 74.38 & 121 \\ \text { X/NORTH WEST } & 26.47 & 73.53 & 34\end{array}$

RESPONDENT'S AGE

$\begin{array}{llll}18-24 & 46.27 & 53.73 & 134 \\ 25-44 & 30.95 & 69.05 & 475 \\ 45 \text { AND OVER } & 23.79 & 75.21 & 391\end{array}$

RESPONOENTIS EDUCATION

$\begin{array}{llll}\text { LT HIGH SCHOOL } & 30.36 & 67.64 & 112 \\ \text { HIGH SCHOOL GRAD } & 27.22 & 72.78 & 371 \\ \text { SOME COLLEGE } & 35.93 & 64.07 & 270 \\ \text { COLLEGE GRAC } & 28.34 & 71.66 & 247 .\end{array}$

RESPONDENT 'S SEX

$\begin{array}{llll}\text { MALE } & 29.86 & 70.14 & 499 \\ \text { FEMALE/HOME } & 30.86 & 69.14 & 243 \\ \text { FEMALE/EMPLOYED } & 30.23 & 69.77 & 258\end{array}$

PRIMARY SOURCE OF FAMILY INCOME/C

$\begin{array}{lllr}\text { SLF-EMPL/WHITE C } & 32.79 & 67.21 & 430 \\ \text { BLUE-COLLAR } & 27.57 & 72.43 & 243 \\ \text { NOT EMPLOYED } & 27.70 & 72.30 & 213 \\ \text { OTHER } & 30.70 & 69.30 & 114 \\ \text { AGGREGATE } & 30.20 & 69.80 & 1000\end{array}$


TABLE 23 (continued)

74

TARRANCE \& ASSC. - SAFETY GELT (MOOO) - \#2017

P44/DRIVING HABITS CHANGED IN THE LAST

YES UNSURE TOTAL

USER CATAGORIES

NOT $\triangle P P L I C A B L E$

FREQ USERS/CNFRM

SMTM USEP/LIKELY

SMTM USER/UNLKLY

30.23

31.17

69.77

$31 i$

68.83

$7 ! .74$

28.26

INFREO USR/LIKLY

33.93

65.07

$27.81 \quad 72.19$

231

92

168

INFREO USR/CNFRM

151

P65/WHEPE DO YOU LIVE

IV A BIG CITY

SUBUPBS/BIS CITY

IN A SMALL CITY

TOWN/VILLAGE

IV THE COUNITRY

CK/NO ANSWER

FREQ-i]RV-1 HR DRNKNG

DAILY TO I/MONTH

SEVERAL TMS/YR

REVER

AGGRESATE
33.86

33.10

30.36

24.71

25.20

33.33
56.14

66.90

69.64

75.29

74.80

65.67

69.50

69.95

69.85

69.80
189

287

224

170

127

3

$\begin{array}{lll}30.50 & 69.50 & 200 \\ 30.05 & 69.95 & 193 \\ 30.15 & 69.85 & 607 \\ 30.20 & 69.80 & 1000\end{array}$


TABLE 24 - DROVE MORE/LESS THIS HOLIDAY SEASON

TARRANCE \& ASSC. - SAFETY BELT (MOOO) - \#20.17

P4T/USE CAR MORE/LESS DURING HOLIDAY SE

MORE LESS THE SAME UNSURE TOTAL

STANDARO FED. ADMNSTRATV REGIONS

$\begin{array}{lllllr}\text { I/NEW ENGLND STS } & 16.67 & 22.22 & 55.56 & 5.56 & 54 \\ \text { II/NW YRKINW JRY } & 15.18 & 25.00 & 50.89 & 8.93 & 112 \\ \text { III/MIO ATLANTIC } & 17.14 & 30.48 & 49.52 & 2.86 & 105 \\ \text { IV/SOUTH EAST } & 14.94 & 32.18 & 47.70 & 5.17 & 174 \\ \text { VIGREAT LAKES } & 17.73 & 39.41 & 38.92 & 3.94 & 203 \\ \text { VI/MID SOUTH } & 21.43 & 36.61 & 38.39 & 3.57 & 112 \\ \text { VII/PLAINS } & 18.87 & 41.51 & 39.62 & 0.00 & 53 \\ \text { VIIINN PLAINS } & 12.50 & 34.38 & 53.13 & 0.00 & 32 \\ \text { IX/WEST COAST } & 14.88 & 37.19 & 45.45 & 2.48 & 121 \\ \text { XINORTH WEST } & 20.59 & 38.24 & 38.24 & 2.94 & 34\end{array}$

RESPONOENT'S AGE

$\begin{array}{llllll}18-24 & 42.54 & 29.10 & 24.63 & 3.73 & 134 \\ 25-44 & 17.05 & 38.32 & 41.68 & 2.95 & 475 \\ 45 \text { ANO OVER } & 7.93 & 30.43 & 56.01 & 5.63 & 391\end{array}$

RESPONOENTIS EOUCATION

$\begin{array}{lrrrrr}\text { LT HIGH SCHOOL } & 8.93 & 35.71 & 48.21 & 7.14 & 112 \\ \text { HIGH SCHOOL GRAD } & 17.52 & 33.96 & 44.47 & 4.04 & 371 \\ \text { SOME COLLEGE } & 19.26 & 34.81 & 42.22 & 3.70 & 270 \\ \text { COLLEGE GRAD } & 17.00 & 32.39 & 47.37 & 3.24 & 247\end{array}$

RESPONOENTIS SEX

$\begin{array}{lccccc}\text { MALE } & 17.23 & 32.06 & 46.59 & 4.01 & 499 \\ \text { FEMALE/HOME } & 13.99 & 37.45 & 44.86 & 3.70 & 243 \\ \text { FEMALE/EMPLOYED } & 18.99 & 34.50 & 41.86 & 4.65 & 258 \\ \text { PRIMARY SOURCE OF FAMILY INCOME/C } & & & \\ & 17.91 & 32.79 & 46.28 & 3.02 & 430 \\ \text { SLF-EMPL/WHITE C } & 17.52 & 36.63 & 41.98 & 2.88 & 243 \\ \text { SLUE-COLLAR } & 18.80 & 7.51 & 213 \\ \text { NOT EMPLOYEO } & 10.80 & 33.80 & 47.89 & 4.39 & 114 \\ \text { OTHER } & 21.05 & 33.33 & 41.23 & 4.39 & \\ \text { AGGREGATE } & 16.90 & 34.00 & 45.00 & 4.10 & 1000\end{array}$


TABLE 24 (continued)

TARRANCE S ASSC. - SAFETY BELT (MOOD) - \#2017

P4T/USE CAR MORE/LESS UURING HOLIOAY SE MORE LESS THE SAME UNSURE TOTAL

USER CATAGORIES

NOT APPLICABLE

FREO USEPS/CNFRM

SMTM USER/LIKELY

SMTM USER NUNLKLY

INFREO USR/LIKLY

INFREQ USR/CNFRM

$\begin{array}{ll}17.68 & 36.0 i \\ 15.58 & : \\ 21.74 & 38.10 \\ 15.67 & 30.43 \\ 17.22 & 29.17 \\ 29.80\end{array}$

42.77
44.16
46.74
47.02
47.02

3.54
2.16
1.09
7.14
5.96

$31 i$

231

92

168

151

P65/WHERE DO YOU LIVE

IN $\triangle$ BIG CITY
SUBURBS/BIG CITY
IN A SMALL CITY
TONN/VILLAGE
IN THE COUNTRY
CK/NO ANSWER

FREO-ORV-1 HR ORNKNG

DAILY TO I/MONTH
SEVERAL TMS/YR
NEVER
AGGREGATE.

AGGREGATE

FREO-ORV-I HR ORNKNG

$\begin{array}{rr}18.52 & 38.10 \\ 14.98 & 32.40 \\ 20.98 & 31.70 \\ 15.29 & 32.94 \\ 14.17 & 36.22 \\ 0.00 & 65.67\end{array}$

39.15

47.04

44.20

48.24

47.24

0.00

4.23

5.57

3.13

3.53

2.36

33.33

189

287

224

170

127

3

$23.50 \quad 33.50$

41.50

1.50

200

$18.65 \quad 29.53$

49.74

$14.17 \quad 35.58$

44.65

2.07

193

5.60

607

$16.90 \quad 34.00$

45.00

4.10

1000 
TABLE 25

CODES - WHY USE CAR MORE THIS H'JLIDAY

And why did your family use your car more this holiday season than the previous one?

01. VISITING RELATIVES - we went to visit my sister more/my daughter lives out of state/we went to visit family/we visited relatives more/our family lives farther away/

02. TOOK A TRIP - because we took a trip to Indiana/we took a trip to Georgia during the holidays/we traveled East for Christmas/went out of town/

03. VISITING/VISITING FRIEND\$ - we did more visiting this holi- (14\%) day season/we went visiting with holiday guests/more people to see/we were visiting friends/some of our friends moved close enough to drive to/

04. MORE PLACES TO GO - more places to go/there was more to do this year/we had more parties to attend/more to do/because we went out more. to different places/

05. JOB RELATED - I got a part-time job/I had to work/I have a new job and drive to Jersey City every weekend/I was further from my job last season/

06. SHOPPING - more shopping trips/went shopping more/I had more shopping to do this holiday season/

07. SCHOOL RELATED - I was in school and was driving back and forth/because I go to school/more college trips/

08. MEDICAL RELATED - because I went to the doctor more/because ( $3 \%$ ) my wife was hospitalized and. I was going back and forth to see her/

09. BETTER WEATHER - the weather was nicer so we went on more trips/the weather was better/

10. MORE TIME/MONEY - more free time/we weren't as busy so we had time to go to more social outings/I was not working and I had more time for useless trips/my husband was off on weekends/I had more money/

11. QUT-OF-TOWN GUESTS - we had out-of-town company and went out more/because we had out-of-town guests and we took them out showing them around/my sister was visiting and went to take her sightseeing/

12. MORE/BETTER CARS - we had an additional car to use/better car/our car was working this season/we only had one car and now we have two, so we're both driving/ 
TABLE 25 (continued)

13. MOVED - we were in the process of moving to Idaho/I moved/ ( $2 \%$ ) we moved during the holiday season/

14. MORE DRIVERS - more people could drive in the family/more (2\%) people driving/I had my license/

15. MARITAL STATUS - because I was single/change of marital status/

16. OTHER

17. DON'T KNOW/NO ANSWER 
And why did your family use your car less this holiday season than the previous one?

01. STAYED HOME - we didn't go any $p 1$ ace/we stayed home this year/just didn't/I just don't go anywhere/family stayed home more this year/we just celebrate at home/

02. OTHERS CAME HERE - company came over/everybody came to our house for the holidays/my mother came here instead of our going there/it was my turn to have everyone/rotating family Christmas/

03. TO0 EXPENSIVE - we were broke/cutting back to save money/ money shortage/too expensive/the poor economic situation/ less money to go places/

04. STAYED LOCAL - we didn't go away on a trip/I stayed in the local area/didn't go out of town/we just went a block/we just stayed with neighbors/.

FAMILY ILLNESS/ACCIDENTS - I was in traction for 6 months/ we were both sick/we had some colds/I broke my leg/Mom was ill/

06. FAMILY CLOSER NOW - family a couple of miles away from home/our family was pretty close by/the family was a lot closer than before/most of our family has moved closer to us/

07. LESS SOCIALIZING - we didn't go out as much/wasn't no one at home/I don't have as much demand socially/attend less social events/I didn't have anywhere to go/

08. WORKED/LESS TIME - wife worked/my job changed and I put in more hours/had to work on the holidays/I have a business and cannot leave it/I didn't have time this year/

09. CAR PROBLEMS/NO CAR - I did not have my car the previous year/the way my car uses up gas/I had an accident and we were without a vehicle for a short while/car trouble/

10. TRAFFIC FEARS - too much holiday traffic/accidents increase ( $5 \%$ ) during the holiday season/so many people on the road who have been drinking/traffic in Florida was bad/

11. WEATHER CONDITIONS - we couldn't get out of town because of bad weather/I don't like to drive through the snow/ weather conditions/there was a blizzard/

12. GAS PRICES/PROBLEMS - because of the gas problem/the higher ( $3 \%$ ) gas prices/gas prices too high/cost of gas/ 
TABLE 26 (continued)

13. TOOK A PLANE/TRAIN/BUS - we flew instead of drove to be' with our family/I went on a plane/we rode the bus/went on a train/

14. DIDN'T VISIT FAMILY/TOO FAR - we didn't go to see my family/didn't go visiting any relatives/didn' $t$ make the usual visit to my parents/didn't go to my brother's house/away from my family ancl relatives/daughters are farther away/

15. UNEMPLOYED - lack of employment/I was unemployed this year/ ( $1 \%$ ) my husband was iaid of $f / I$ quit work/

16. ELDERLY/RETIRED - too old to go anymore/we're retired/it's due to my age/

17. CHANGED SHOPPING HABITS - I didn't take long shopping trips/I shopped earlier/didn't drive around Christmas shopping as much/we have been shopping by mail/I don't shop with my wife anymore/do less shopping/

18. OTHERS DROVE - someone else drove/traveled with son and his family/a friend driving instead of myself/traveled with other people/

19. NOT HOME - we were out of state/stayed in New York all through the holidays/we were in California/I was overseas and didn't drive much/

20. DEATH IN FAMILY - my mother died/death in family/I was by myself due to my husband passing away/

21. ALTERED LIFE STYLE - married this year/I was pregnant/the baby was just born a week before Christmas/we moved/we relocated from the North to the South/

22. OTHER

23. DON'T KNOW/NO ANSWER 
These results may indicate that it is the non-drinking driver who is staying off the road at the high risk times rather than the drinking driver. In order to develop definitive trend data, these questions will have to be asked on a continuing basis. The data from this survey provide only the starting point. 


\section{FIGURE 15 - AGE BY CHANGE DRIVING HABITS BY FREOUENCY DRINK/DRIVE}

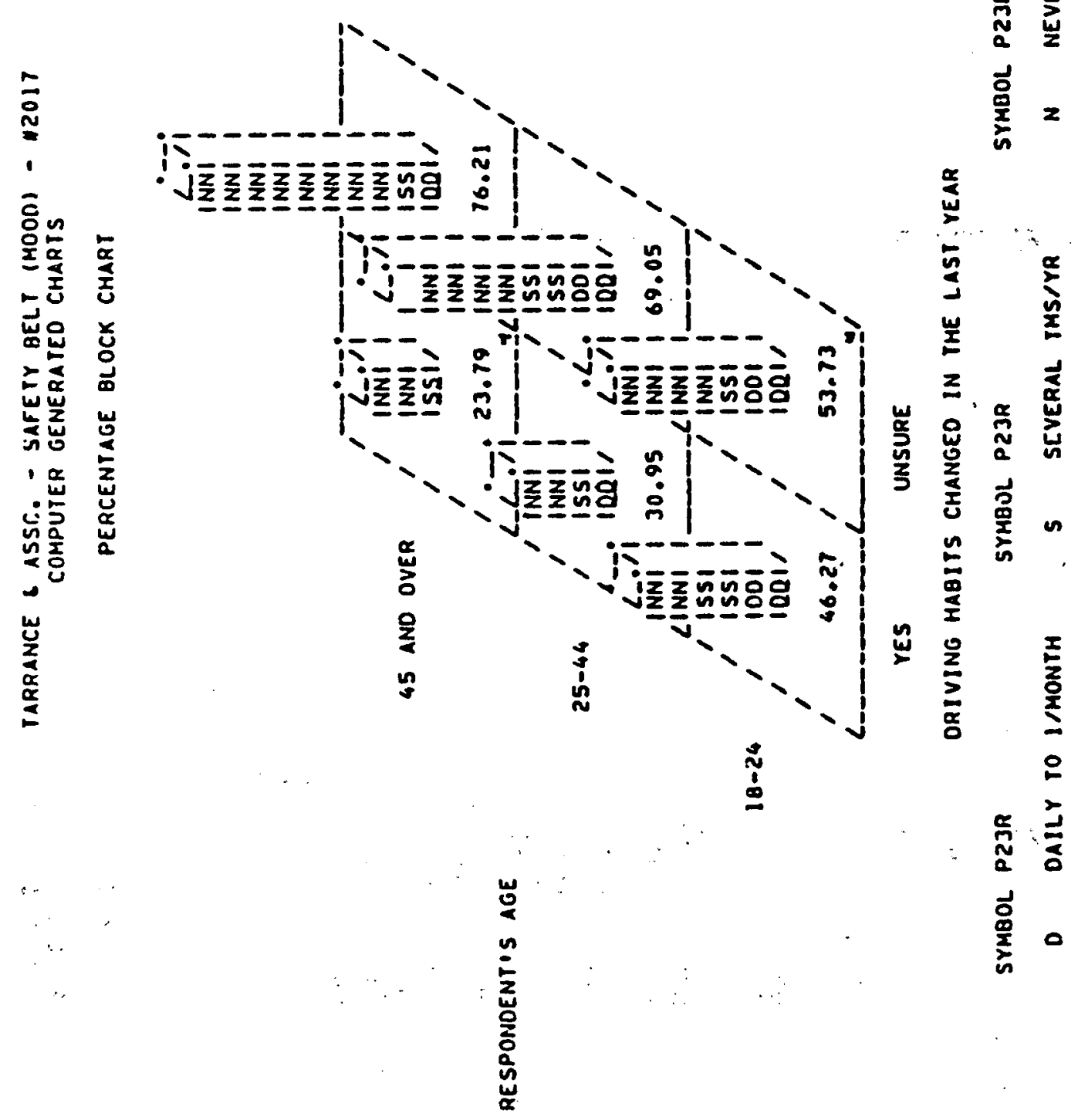


FIGURE 16 - AGE BY CAR USE HOLIDAYS BY FREQUENCY DRINK/DRIVE

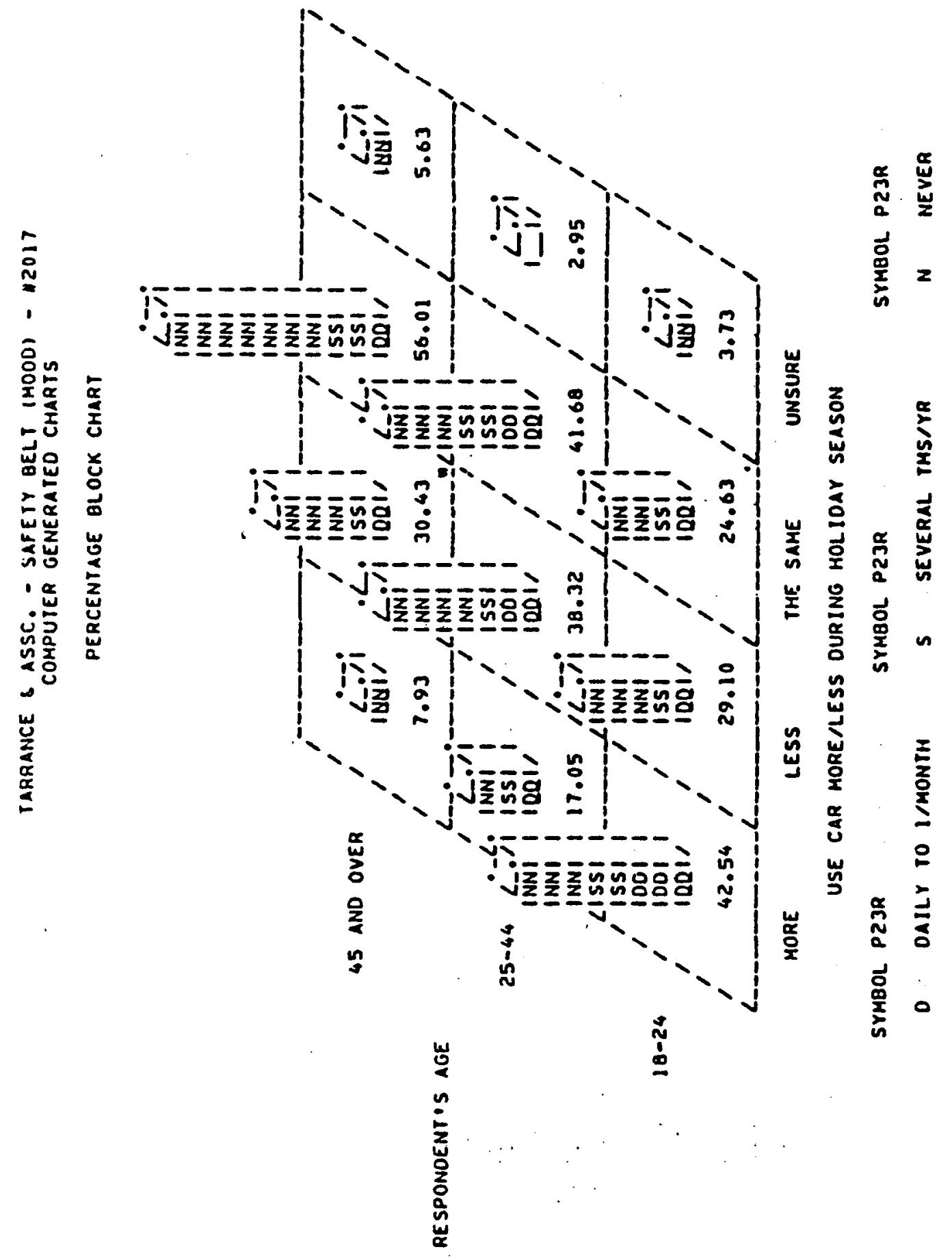



FIGURE 17 - AGE BY CAR USE VS. LAST YEAR BY FREQUENCY DRINK/. DRIVE

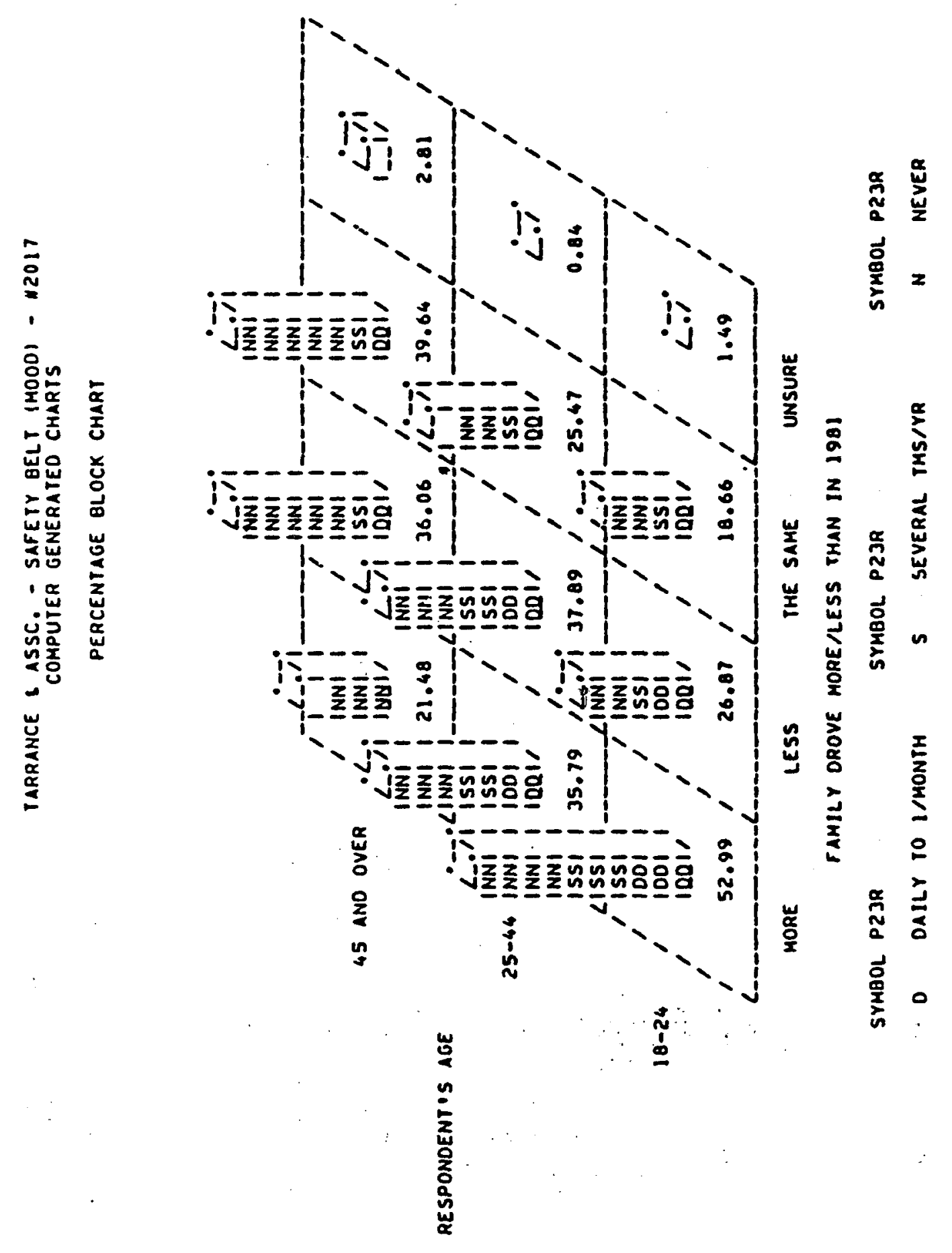


FIGURE 18 - FREOUENCY DRINK/DRIVE BY CAR USE HOLIDAYS BY AGE

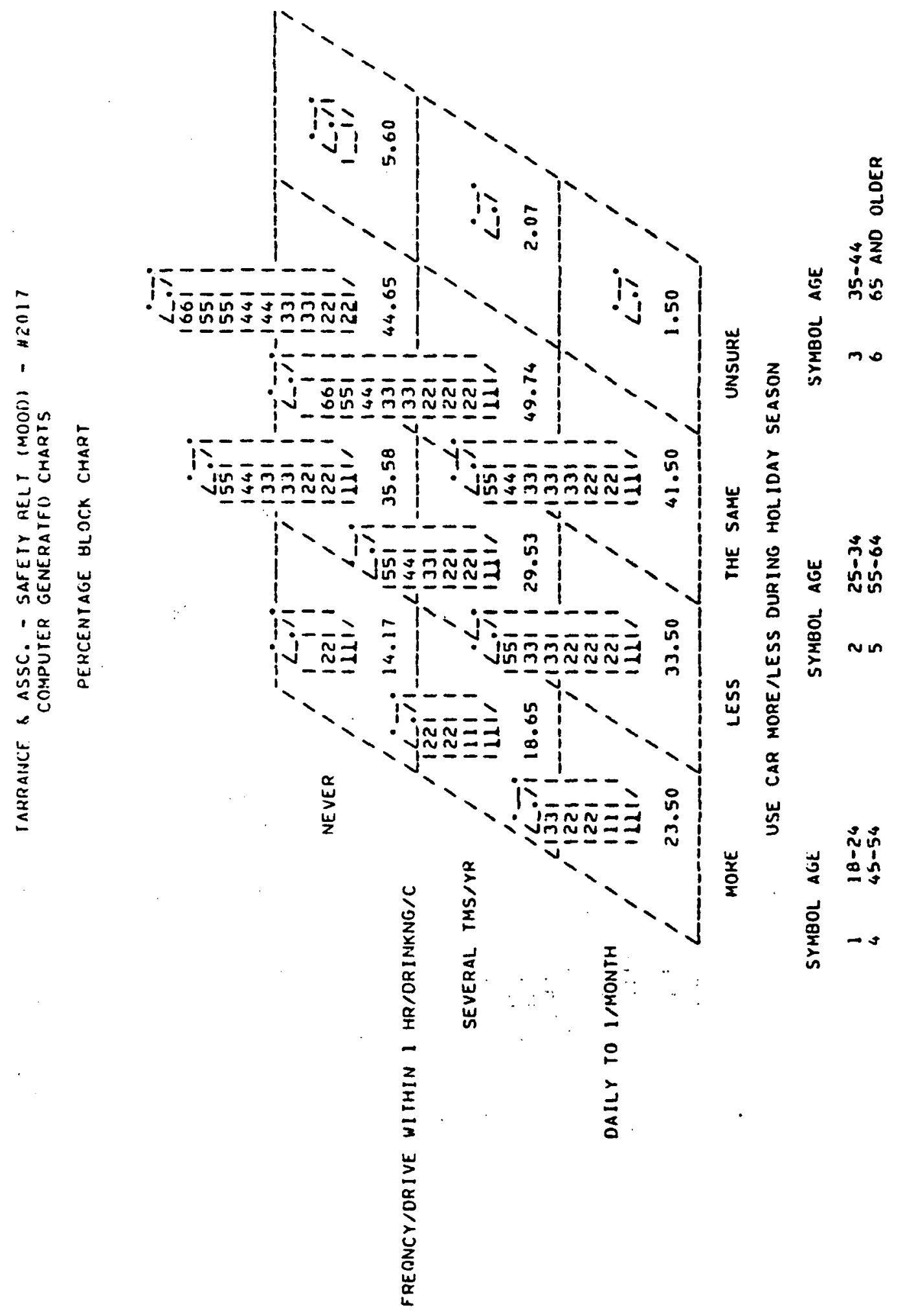


TABLE 27A

VERBATIMS - WHY DROVE MORE IN 1982

Question 43-1:

And why did your family drive more in 1982?

\begin{tabular}{lllll}
\hline $\begin{array}{l}\text { Geographic } \\
\text { Region }\end{array}$ & Age & Education & Sex & Categories \\
\hline VI/ & & $\begin{array}{l}\text { College } \\
\text { Graduate }\end{array}$ & Male & $\begin{array}{l}\text { Friquent/ } \\
\text { Confirmed }\end{array}$
\end{tabular}

"Because in 1981 we lived in Korea and there weren't many places to drive."

$\begin{array}{llll}\text { IX/ } & \text { Some } & \text { Frequent/ } \\ \text { West coast } & 25-34 & \text { College } & \text { Male } \\ \text { Confirmed }\end{array}$

"My changes in business practices causes me to drive more."

V/ High School Female/ Infrequent/

Great Lakes 25-34 Graduate Home Confirmed

"We took a family trip to areas we'd never been before...it was a family reunion."

$\begin{array}{lllll}\text { VIII/ } & 65 \& & \text { College } & \begin{array}{l}\text { Female/ } \\ \text { Graduate }\end{array} & \begin{array}{l}\text { Frequent/ } \\ \text { Confirmed }\end{array} \\ \text { N.W. Plains } & \text { Over } & \text { Graduate } & \text { Home }\end{array}$

"My children have moved to different states and I go to visit them."

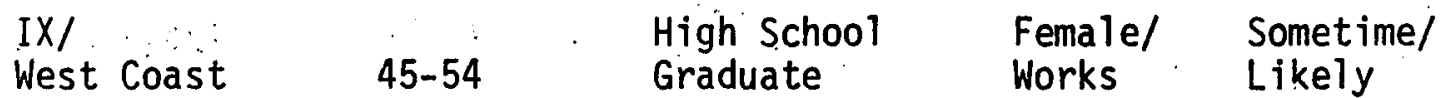

"My husband retired and we had more time to go places."

$\begin{array}{llll}\text { VI/ } & \text { Less than } & \text { Frequent/ } \\ \text { Mid South } & 45-54 & \text { High School Male } & \text { Confirmed }\end{array}$

"The lower gasoline prices... we took more drives after work because the gasol ine prices were lower."

IV/

South East
High School

Graduate
Female/ Works
Infrequent/

Likely

"My daughter got her license so we have another driver in the family." 
TABLE 27A (continued)

Question 43-1 continued:

\begin{tabular}{lllll}
\hline $\begin{array}{l}\text { Geographic } \\
\text { Region }\end{array}$ & Age & Education & Sex & Categories \\
\hline $\begin{array}{l}\text { V/ } \\
\text { Great Lakes }\end{array}$ & $18-24$ & $\begin{array}{l}\text { Some } \\
\text { College }\end{array}$ & Male & $\begin{array}{l}\text { Sometime/ } \\
\text { Likely }\end{array}$
\end{tabular}

"I'm in school so I live several miles away from my family and we like to keep together and visit each other."

X/

North West

$45-54$

High School

Graduate
Female/

Home
Infrequent/

Confirmed

"We bought a motor home and my husband retired and we're seeing the United States."

IV/

South East

25-34

High School

Graduate

Female/

Sometime/

Home

Unlikely

"We vacationed more...we moved to where we are now and my husband was home more so we were on the go more." 
TABLE 27B

VERBATIMS - WHY DROVE LESS IN 1982

Question 43-2:

And why did your family drive less in 1982 ?

\begin{tabular}{|c|c|c|c|c|}
\hline $\begin{array}{l}\text { Geographic } \\
\text { Region }\end{array}$ & Age & Education & Sex & $\begin{array}{l}\text { User } \\
\text { Categories }\end{array}$ \\
\hline Mid Atlantic & $25-34$ & $\begin{array}{l}\text { High School } \\
\text { Graduate... }\end{array}$ & Male & $\begin{array}{l}\text { Infrequent/ } \\
\text { Confirmed }\end{array}$ \\
\hline \multicolumn{5}{|c|}{$\begin{array}{l}\text { "Gas prices... the economy... there wasn't that many places we could afford to } \\
\text { go." }\end{array}$} \\
\hline $\begin{array}{l}\text { III/ } \\
\text { Mid Atlantic }\end{array}$ & $\begin{array}{l}65 \& \\
\text { Over }\end{array}$ & $\begin{array}{l}\text { Less than } \\
\text { High School }\end{array}$ & Male & $\begin{array}{l}\text { Sometime/ } \\
\text { Likely }\end{array}$ \\
\hline
\end{tabular}

$\begin{array}{llll}\text { IV/ } & 65 \& & \begin{array}{l}\text { High School } \\ \text { Graduate }\end{array} & \begin{array}{l}\text { Sometime/ } \\ \text { Likely }\end{array}\end{array}$

"Used to take wife farther for dialysis treatment but now only take her a short distance."

$\begin{array}{llll}\text { IV/ } & \text { Some } & \text { Mnfrequent/ } \\ \text { South East } & 35-44 & \text { College } & \begin{array}{l}\text { Infe } \\ \text { Likely }\end{array}\end{array}$

"We both had jobs and were working and didn't have time to take a trip last year."

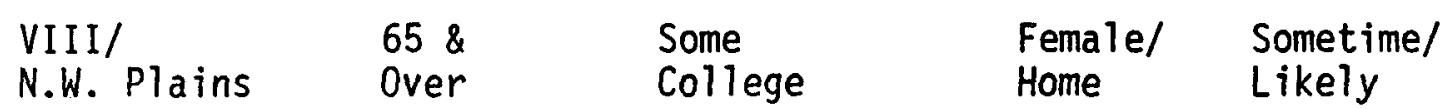

"I go on the train a lot now... I don't like to drive long distances now that I'm retired."

VII/

Plains
High School

Graduate
Infrequent/

Male Confirmed

"I was transferred closer to home on my job."

$\begin{array}{llll}\text { IV/ } & \text { High School } & \begin{array}{l}\text { Female/ } \\ \text { Gome }\end{array} & \begin{array}{l}\text { Sometime/ } \\ \text { Likely }\end{array}\end{array}$

"I have a different type of car now and I'm afraid to take it on long trips." 
TABLE 27B (continued)

Question 43-2 continued:

\begin{tabular}{|c|c|c|c|c|}
\hline $\begin{array}{l}\text { Geographic } \\
\text { Region }\end{array}$ & Age & Education & Sex & $\begin{array}{l}\text { User } \\
\text { Categories }\end{array}$ \\
\hline $\begin{array}{l}\text { II/New York/ } \\
\text { New Jersey }\end{array}$ & $45-54$ & $\begin{array}{l}\text { Less than } \\
\text { High School }\end{array}$ & $\begin{array}{l}\text { Female/ } \\
\text { Home }\end{array}$ & $\begin{array}{l}\text { Infrequent/ } \\
\text { Likely }\end{array}$ \\
\hline
\end{tabular}

"Because we usually drive around on our snow mobile but since it didn't snow much we couldn't ride in it very much."

V/

Great Lakes

$65 \&$

High School

Female/

Infrequent/

Home

Confirmed

"Our circumstances were different then... we had a resort place quite a ways from our home... we've bought one closer now."

II N New York/. New Jersey $25-34$
Some

College
Female/ Frequent/ Works Confirmed

"Because in 1981 I was pregnant and we had to go to the hospital every week." 
TABLE 28A

VERBATIMS - WHY DROVE MORE THIS HOLIDAY

Question 48-1:

And why did your family use your car more this holiday season than the previous one?

\begin{tabular}{lllll}
\hline $\begin{array}{l}\text { Geographic } \\
\text { Region }\end{array}$ & Age & Education & Sex & User \\
\hline & & Categories \\
\hline IV/ & $25-34$ & $\begin{array}{l}\text { College } \\
\text { Gouth East }\end{array}$ & Male & $\begin{array}{l}\text { Frequent/ } \\
\text { Confirmed }\end{array}$ \\
"Had relatives that had moved a great distance." &
\end{tabular}

V/

Great Lakes

$18-24$
High School

Graduate
Female/ Infrequent/

Works Confirmed

"Visited people this year instead of them visiting us."

IV/

South East
High School

Graduate
Frequent/

Male Confirmed

"Because I had a different job and had to use the car more.".

I/New England

States

$18-24$

High School

Graduate

Male

Frequent/

Confirmed

"Christmas shopping made us do more driving... we finally had a little bit of extra money."

IV/

South East

$18-24$

Some

College
Frequent/

Male Confirmed

"I I ive in New York and go to school in Louisiana... I drove back and forth a few times."

III/

Mid Atlantic
High School

Graduate
Female/ Frequent/

Home Confirmed

"We went more because the weather was better." 
TABLE 28A (continued)

Question 48-1 continued:

\begin{tabular}{|c|c|c|c|c|}
\hline $\begin{array}{l}\text { Geographic } \\
\text { Region }\end{array}$ & Age & Education & Sex & $\begin{array}{l}\text { User } \\
\text { Categories }\end{array}$ \\
\hline $\begin{array}{l}\text { X } \\
\text { North West }\end{array}$ & $35-44$ & $\begin{array}{l}\text { High School } \\
\text { Graduate }\end{array}$ & $\begin{array}{l}\text { Female/ } \\
\text { Works }\end{array}$ & $\begin{array}{l}\text { Frequent/ } \\
\text { Confirmed }\end{array}$ \\
\hline $\begin{array}{l}\text { V/ } \\
\text { Great Lakes }\end{array}$ & $25-34$ & $\begin{array}{l}\text { High School } \\
\text { Graduate }\end{array}$ & $\begin{array}{l}\text { Female/ } \\
\text { Works }\end{array}$ & $\begin{array}{l}\text { Sometime/ } \\
\text { Likely }\end{array}$ \\
\hline
\end{tabular}


TABLE 28B

VERBATIMS - WHY DROVE LESS THIS HOLIDAY

Question 48-2:

And why did your family use your car less this holiday season than the previous one?

\begin{tabular}{lllll}
\hline $\begin{array}{l}\text { Geographic } \\
\text { Region }\end{array}$ & Agge & Education & Sex & User \\
\hline V/ & Categories \\
Great Lakes & $25-34$ & $\begin{array}{l}\text { High School } \\
\text { Graduate }\end{array}$ & Male & $\begin{array}{l}\text { Sometime/ } \\
\text { Unlikely }\end{array}$
\end{tabular}

"It was my turn to have everyone over here for the holiday so we didn't go out."

VIII/

N.W. Plains
High School

Graduate
Female/.

Home
Sometime/

Likely

"We all had to work more so we didn't have the time or the opportunity to this year."

IVI

South East
Less than

High School
Sometime/

Male. Likely

"I don't like being on the roads and highways during holidays and at high traffic times."

VI/

Mid. South

$65 \&$

Over
College

Graduate
Sometime/

Male
Unlikely

"We didn't want to drive on the highway with all those drunks."

V/

Great Lakes

$55-64$

Some

"Because it was winter time and I don't like to drive through the snow."

III/

Mid Atlantic
College

Graduate
Female/

Works
Sometime/

Unlikely

"Lack of employment and thereby less money and less social engagements." 
TABLE 28B (continued)

Question 48-2 continued:

\begin{tabular}{lllll}
\hline $\begin{array}{l}\text { Geographic } \\
\text { Region }\end{array}$ & Age & Education & Sex & User \\
\hline V' & & $\begin{array}{l}\text { Some } \\
\text { College }\end{array}$ & Male & $\begin{array}{l}\text { Infrequent/ } \\
\text { Confirmed }\end{array}$
\end{tabular}

"I'm in maintenance so when most people are off, such as during holidays, I'm working."

IV/

South East
Some

$35-44$

College

Infrequent/

Male Likely

"I wanted to stay closer to my family this year...my son was away in the Navy."

VI/

Mid South

Some

"I was on the road all the time in 1982 and during the holidays I stayed home." 
PERCEPTION OF RISK AS AN ISSUE IN THE USE

OF SAFETY BELTS AND DRIVING AFTER DRINKING

There are two themes which are very prevalent when the topic of perception of risk in driving is addressed in a focus group. These are control and danger. In fact, for many respondents, the danger is the major component of risk.

A number of questions were asked to get at the issue of perception of risk and its utility as a motivator to either increase the use of belts and/or reduce the prevalence of DWI. For purposes of this discussion, these questions will be lumped into two groups - Control and Risk.

$\underline{\text { Risk }}$

Table 29 presents the data for the question "People are more likely to put their seat belts on if they think that driving conditions are more dangerous than usual". In general, about $78 \%$ of the general population agrees with this statement. There are, however, a few demographic groupings which do not fall into the general population trend. They are the less than high school groups and the infrequent wearers, both of which are well below the general population. The "sometimes users unlikely" are well above the general population. This suggests that their reason for using the belt may, in a large part, be based on a perception of risk or danger. When the general population is broken into the three alcohol groups, no differences appear.

Another question which approaches the issue in a different way is:

"Mr. Cooper does not wear seat belts because he thinks he is a good driver and won't be in an accident."

"Mr. Lewis wears his seat belts because he isn't sure about the other drivers and just doesn't like to take chances." 
95

TABLE 29 - DEMOGRAPHICS BY WEAR BELTS WHEN CONDITIONS DANGEROUS

TARRANCE \& ASSC. - SAFETY BELT (MOOD) - \#2017

PIO/WEAR BELTS IF DRVNG CNDTNS ARE DANG

AGREE/ST AGREE UNSURE DISAGREE OISAGREE
RONGLY ISTRNGLY

TOTAL

STANDARD FED. ADMNSTRATV REGIONS

$\begin{array}{llllrrr}\text { I/NEW ENGLND STS } & 51.85 & 19.52 & 7.41 & 7.41 & 14.81 & 54 \\ \text { II/NW YRK/NW JRY } & 47.32 & 29.46 & 6.25 & 12.50 & 4.46 & 112 \\ \text { III/MID ATLANTIC } & 53.33 & 20.00 & 6.67 & 11.43 & 8.57 & 105 \\ \text { IV/SOUTH EAST } & 52.30 & 24.71 & 9.20 & 8.62 & 5.17 & 174 \\ \text { V/GREAT LAKES } & 52.71 & 25.62 & 4.43 & 9.85 & 7.39 & 203 \\ \text { VI/MIO SOUTH } & 48.21 & 31.25 & 7.14 & 5.36 & 8.04 & 112 \\ \text { VII/PLAINS } & 54.72 & 25.42 & 1.89 & 3.77 & 13.21 & 53 \\ \text { VIII/NW PLAINS } & 59.38 & 231.25 & 10.01 .00 & 3.13 & 6.25 & 32 \\ \text { IX/WEST COAST } & 51.24 & 29.75 & 3.31 & 9.92 & 5.79 & 121 \\ \text { X/NORTH WEST } & 41.18 & 38.24 & 2.94 & 11.76 & 5.88 & 34\end{array}$

RESPONDENTIS AGE

$\begin{array}{lllllll}18-24 & 51.49 & 25.12 & 2.99 & 11.94 & 7.46 & 134 \\ 25-44 & 52.21 & 28.00 & 4.42 & 8.00 & 7.37 & 475 \\ 45 \text { ANO OVER } & 50.13 & 25.32 & 8.18 & 9.21 & 7.16 & 391\end{array}$

RESPONOENT'S EDUCATION

$\begin{array}{lrrrrrr}\text { LT HIGH SCHOOL } & 44.64 & 23.21 & 16.07 & 7.14 & 8.93 & 112 \\ \text { HIGH SCHOOL GRAD } & 52.02 & 26.68 & 5.66 & 7.28 & 8.36 & 371 \\ \text { SOME COLLEGE } & 53.70 & 24.44 & 3.33 & 12.22 & 6.30 & 270 \\ \text { COLLEGE GRAD } & 50.61 & 30.77 & 3.64 & 8.91 & 6.07 & 247\end{array}$

RESPONDENT'S SEX

$\begin{array}{lllllll}\text { MALE } & 48.10 & 27.05 & 6.41 & 10.62 & 7.82 & 499 \\ \text { FEMALE/HOME } & 56.38 & 27.98 & 3.29 & 5.35 & 7.00 & 243 \\ \text { FEMALE/EMPLOYED } & 52.71 & 24.81 & 6.59 & 9.30 & 6.59 & 258\end{array}$

PRIMARY SOURCE OF FAMILY INCOME/C

\begin{tabular}{|c|c|c|c|c|c|c|}
\hline $\begin{array}{l}\text { SLF -EMPL/WHITE C } \\
\text { BLUE-COLLAR } \\
\text { NOT EMPLOYED } \\
\text { OTHER }\end{array}$ & $\begin{array}{l}53.72 \\
51.44 \\
46.01 \\
51.75\end{array}$ & $\begin{array}{l}27.91 \\
23.87 \\
27.70 \\
26.32\end{array}$ & $\begin{array}{l}4.88 \\
4.53 \\
7.98 \\
7.02\end{array}$ & $\begin{array}{r}7.67 \\
12.35 \\
8.92 \\
7.02\end{array}$ & $\begin{array}{l}5.81 \\
7.82 \\
9.39 \\
7.89\end{array}$ & $\begin{array}{l}430 \\
243 \\
213 \\
114\end{array}$ \\
\hline AGGREGATE & 51.30 & 25.70 & 5.70 & 9.00 & 7.30 & 1000 \\
\hline
\end{tabular}


TABLE 29 (continued)

TARRANCE \& ASSC. - SAFETY BELT (MOOD) - \#2017

PIO/WEAR BELTS IF ORVNG CNDTNS ARE DANG

AGREE/ST AGREE UNSURE DISAGREE OISAGREE
RONGLY ISTRNGLY

TOTAL

USER CATAGORIES

NOT $\triangle P P L I C A B L E$

FREO USERS/CNFRM

SMTM USER/LIKELY

SMTM USER/UNLKLY

INFREQ USR/LIKLY

INFREQ USR/CNFRM

$\begin{array}{rrr}50.80 & 28.62 & 4.18 \\ 61.90 & 22.94 & 2.60 \\ 6.7 .39 & 22.83 & 0.00 \\ 45.83 & 25.60 & 7.14 \\ 37.09 & 30.46 & 12.58\end{array}$

$\begin{array}{rr}7.40 & 9.00 \\ 6.49 & 6.06 \\ 6.52 & 3.026 \\ 14.88 & 6.55 \\ 10.60 & 9.27\end{array}$

$3 ! i$

231

9.2

168

151

P65/WMERE DO YOU LIVE

IN $\triangle B I G$ CITY
SUBURBS/EIG CITY
IN A SMALL CITY
TOWN VILLAGE
IN THE COUNTRY

DK/NO GNSWER
48.15

52.26

53.57

49.41

53.54

0.00
25.98

26.48

25.45

28.24

25.77

33.33
5.92

5.92

3.57

7.06

7.09

0.00
8.99
10.45
8.93
8.82
4.72
66.67

10.05

4.88

8.48

6.47

7.87

0.00
189

287

224

170

127

3

FREQ-DPV-1 HR DRNKNG

$\begin{array}{lllllll}\text { DAILY TO I/MONTH } & 51.50 & 31.00 & 4.50 & 8.00 & 5.00 & 200 \\ \text { SEVEPAL TMS/YR } & 52.33 & 24.87 & 3.63 & 13.99 & 5.18 & 193 \\ \text { NEVER } & 50.91 & 25.86 & 6.75 & 7.74 & 8.73 & 607 \\ \text { AGGREGATE } & 51.30 & 25.70 & 5.70 & 9.00 & 7.30 & 1000\end{array}$


The impressive aspect of this data (see Table 30) is how the five SRU groups broke out. This item may be the best item for differentiating the groups and may well pinpoint one of the major factors that differentiates wearers from non-wearers in the area of risk.

In the alcohol area, the isslue of risk was addressed in a slightly different manner and two related questions were asked. The first was:

"Think for a moment about a situation in which you drive within one hour of having had too much to drink. In your own estimation, if you did this 100 times, how many times would you be caught and punished?"

The second question was:

"How high would the number have to be in order for you to decide not to drive after having had too much to drink?"

In each case, the exact number given by the respondent was recorded. Table 31, column I presents the data for the first question and column II for the second question. For both questions, the "don't know" response was about the same (e.g., 15-16\%). In terms of getting caught, about $50 \%$ of the respondents thought their chance of getting caught would be less than $6 \%$. When asked how high the percentage of getting caught would have to be to stop them from driving after drinking, over $50 \%$ said $1 \%$ or 1 out of 100 . Clearly, these data are in conflict in that the reported risk factor for stopping of $1 \%$ is less than the perceived risk of getting caught, which is $6 \%$. In reality, the best estimate of the probability of getting caught is 1 in 2000 , which is significantly less than $1 \%$.

In terms of the perception of risk in getting apprehended while driving under the influence and $i$ ts influence as a motivator to stop people from driving while under the influence further clarification is needed. 
98

TABLE 30 - DEMOGRAPHICS BY GOOD DRIVER/UNSURE OTHER DRIVERS

TARRANCE \& ASSC. - SAFETY BELT (MOOD) - \#2017

PIT/GOOD DRIVER HS. UNSURE OF OTHER DRI
MR COOPR MR COOPE UNSURE MR LEWIS MR LEWIS
ISTRNGLY ISTRNGLY

TOTAL

STANDARD FED. ADMNSTRATV REGIONS

$\begin{array}{lrrrrrr}\text { I/NEW ENGLND STS } & 12.96 & 11.11 & 5.56 & 22.22 & 48.15 & 54 \\ \text { IIINW YRK/NW JRY } & 13.39 & 7.14 & 5.36 & 13.39 & 60.71 & 112 \\ \text { III/MIOATLANTIC } & 8.57 & 7.61 & 4.76 & 19.05 & 60.00 & 165 \\ \text { IV/SOUTH EAST } & 8.62 & 5.17 & 7.47 & 15.52 & 63.22 & 174 \\ \text { V/GREAT LAKES } & 5.42 & 3.94 & 8.37 & 13.79 & 68.47 & 203 \\ \text { VI/MID SOUTH } & 8.93 & 12.50 & 7.14 & 14.29 & 57.14 & 112 \\ \text { VII/PLAINS } & 5.66 & 11.32 & 7.55 & 15.09 & 60.38 & 53 \\ \text { VIII/NW PLAINS } & 6.25 & 9.38 & 3.13 & 12.50 & 68.75 & 32 \\ \text { IX/WEST COAST } & 4.13 & 6.61 & 4.13 & 19.83 & 65.29 & 121 \\ \text { XINORTH WEST } & 0.00 & 5.88 & 5.88 & 11.76 & 76.47 & 34\end{array}$

RESPONDENT'S AGE

$\begin{array}{lrlllll}13-24 & 11.19 & 5.72 & 2.24 & 18.66 & 61.19 & 134 \\ 25-44 & 7.58 & 7.79 & 7.58 & 15.16 & 61.89 & 475 \\ 45 \text { AND OVEK } & 6.65 & 6.65 & 6.39 & 15.60 & 64.71 & 391\end{array}$

RESPONDENT'S EDUCATION

$\begin{array}{lrrrrrr}\text { LT HIGH SCHOOL } & 10.71 & 7.14 & 11.61 & 11.61 & 58.93 & 112 \\ \text { HIGH SCHOOL GRAD } & 8.36 & 8.63 & 5.93 & 16.44 & 60.65 & 371 \\ \text { SOME COLLEGE } & 5.56 & 6.67 & 6.67 & 17.04 & 64.07 & 270 \\ \text { COLLEGE GRAD } & 7.69 & 5.67 & 4.45 & 15.38 & 66.80 & 247\end{array}$

RESPONDENT'S SEX

$\begin{array}{lrrrrrr}\text { MALE } & 10.42 & 8.22 & 7.82 & 16.03 & 57.52 & 499 \\ \text { FEMALE/HOME } & 6.17 & 4.94 & 4.12 & 15.23 & 69.55 & 243 \\ \text { FEMALE/EMPLOYED } & 3.88 & 7.36 & 5.81 & 15.89 & 67.05 & 258\end{array}$

PRIMARY SOURCE OF FAMILY INCOME/C

$\begin{array}{lrrrrrr}\text { SLF-EMPL/WHITE } C & 8.14 & 6.28 & 4.88 & 16.51 & 64.19 & 430 \\ \text { BLUE-COLLAR } & 6.58 & 10.29 & 8.64 & 15.23 & 59.26 & 243 \\ \text { NOT EMPLOYED } & 7.98 & 4.69 & 4.69 & 17.37 & 65.26 & 213 \\ \text { OTHER } & 7.89 & 8.77 & 10.53 & 11.40 & 61.40 & 114 \\ & & & & & & \\ \text { AGGREGATE } & 7.70 & 7.20 & 6.40 & 15.80 & 62.90 & 1000\end{array}$


TABLE 30 (continued)

TARPANCE \& ASSC. - SAFETY BELT (MOOD) - $\$ 2017$
P17/GOOD DRIVER VS. UNSURE OF OTHER DRI
IAP COOPR MR COOPE UNSURE MR LEWIS MR LEWIS ISTPNGLY R ISTRNGLY

USER CATAGORIES

NOT $\triangle P P L I C A B L E$

FREO USERS/CNFRM

SMTM USEP/LIKELY

SMTM USER NUNLKLY

INFREQ USR/LIKLY

INFREQ USR/CNFRM

$\begin{array}{rr}1.93 & 1.61 \\ 5.19 & 5.19 \\ 5.43 & 5.43 \\ 10.71 & 12.50 \\ 21.19 & 17.88\end{array}$

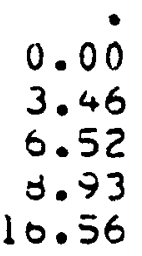

10.29
17.32
15.22
20.24
15.89

86.17

68.83

67.39

47.62

28.40

P65/NHERE DO YOU LIVE

IN A BIG CITY

SUBURBS/BIG CITY

IV A SMALL CITY

TOWN N ILLAGE

IN THE COUNTRY

CK/NO ANSWER
9.52

7.32

5.80

8.82

7.87

0.00

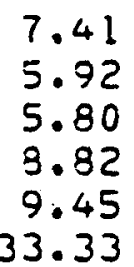

6.35

6.27

4.91

5.88

10.24

0.00
14.81

18.82

15.63

14.12

12.60

33.33
61.90

61.67

67.86

62.35

59.84

33.33
189

287

224

170

127

3

FREO-ORV-I HR DRNKNG

DAILY TO I/MONTH
SEVEPAL TMS/YR
NEVEF.

AGGPEGATE

$\begin{array}{rr}10.50 & 9.50 \\ 7.25 & 5.22 \\ 6.92 & 6.75\end{array}$

7.70

7.20

6.00

4.66

7.08

16.50

14.51

15.98

57.50

67.36

63.26

$6.40 \quad 15.80$

62.90
200

193

607

1000 
TABLE 31

RISK OF PUNISHMENT: DRIVING AND DRINKING

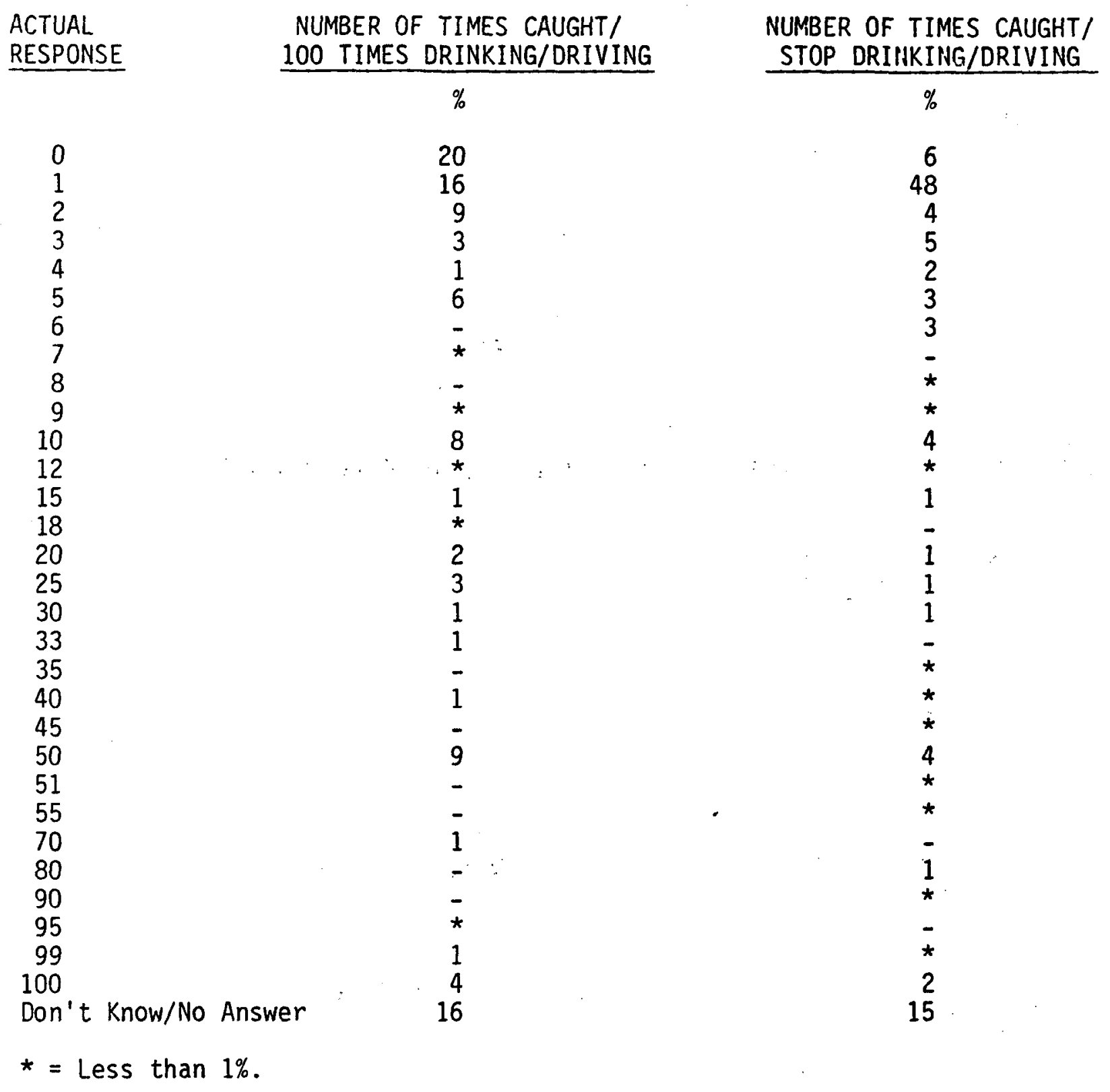




\section{Control}

Four items that were included in the survey that can be included in this category:

1. "People think they won't be in an accident because they are safe drivers in control of their car."

2. "Mr. Thompson feels he has much less control over himself and his car when he drives with his seat belts on."

"Mr. Evans feels he has much more control over himself and his car when he drives with his seat belts on."

3. "How much control do you feel you have in preventing your own involvement in automobile accidents?"

4. "If you were involved in an auto accident, what do you feel would be the most likely cause? Would it be...

1. Your own driving error

2. A drunk driver

3. Someone else's driving error

4. A mechanical defect in the car

5. Unavoidable bad luck

6. Poor road conditions

7. Poor weather conditions

Tables 32 through 35 present the date for each of these questions. The question 'which compares Mr. Thompson's and Mr. Evans' attitudes shows a response set that clearly differentiates between the five user groups. The other three questions do not show any demonstrative differences between groups. The reason for this may be that the other three questions are more general in nature, and do not refer to safety belts. 
102

TABLE 32 - DEMOGRAPHICS BY NO ACCIDENTS/IN CONTROL

TARRANCE \& ASSC. - SAFETY BELT (MOOD) - \#2017

PG/PPLE WON'T HAVE ACCDNTS/IN CNTRL OF

AGREE/ST AGREE UNSURE DISAGREE OISAGREE

PONGLY /STRNGLY

TOTAL

STANDARD FED. ADMNSTRATV REGIONS

$\begin{array}{llllllr}\text { I/NEW ENGLND STS } & 24.07 & 18.52 & 0.00 & 14.81 & 42.59 & 54 \\ \text { II/NW YRKINW JRY } & 17.86 & 16.07 & 2.68 & 27.68 & 35.71 & 112 \\ \text { III/MID ATLANTIC } & 21.90 & 15.24 & 1.90 & 20.95 & 40.00 & 105 \\ \text { IV/SOUTH EAST } & 16.09 & 17.82 & 3.45 & 21.26 & 41.38 & 174 \\ \text { V/GREAT LAKES } & 18.23 & 19.72 & 2.96 & 18.23 & 41.87 & 203 \\ \text { VI/MID SOUTH } & 18.75 & 11.61 & 0.89 & 21.43 & 47.32 & 112 \\ \text { VII/PLAINS } & 16.98 & 13.21 & 3.77 & 15.09 & 50.94 & 53 \\ \text { VIII/NW PLAINS } & 21.88 & 15.63 & 3.13 & 25.00 & 34.38 & 32 \\ \text { IX/WEST COAST } & 23.97 & 15.70 & 1.65 & 21.49 & 37.19 & 121 \\ \text { X/NORTH WEST } & 11.76 & 23.53 & 2.94 & 17.65 & 44.12 & 34\end{array}$

RESPONDENT'S AGE

$\begin{array}{lllllll}18-24 & 18.66 & 17.91 & 0.75 & 23.88 & 38.81 & 134 \\ 25-44 & 21.05 & 19.37 & 1.89 & 19.16 & 38.53 & 475 \\ 45 \text { AND OVER } & 16.38 & 12.53 & 3.58 & 21.48 & 45.52 & 391\end{array}$

RESPONDENT'S EDUCATION

$\begin{array}{lllllll}\text { LT HIGH SCHOOL } & 12.50 & 15.07 & 5.36 & 15.18 & 50.89 & 112 \\ \text { HIGH SCHOOL GRAD } & 18.33 & 13.75 & 1.62 & 22.10 & 44.20 & 371 \\ \text { SOME COLLEGE } & 20.37 & 15.67 & 1.85 & 20.74 & 40.37 & 270 \\ \text { COLLEGE GRAD } & 21.86 & 20.65 & 2.83 & 21.05 & 33.60 & 247\end{array}$

RESPONDENT'S, SEX

$\begin{array}{lcccccr}\text { MALE } & 17.84 & 20.84 & 2.20 & 21.04 & 38.08 & 499 \\ \text { FEMALE/HOME } & 15.05 & 11.93 & 4.12 & 20.58 & 47.33 & 243 \\ \text { FEMALE/EMPLOYED } & 24.42 & 12.40 & 1.16 & 20.16 & 41.86 & 258 \\ \text { PRIMARY SOURCE OF FAMILY INCOME/C } & & & & \\ \text { S F } & & & & & & \\ \text { SLF-EMPL/WHITE C } & 21.86 & 16.74 & 2.09 & 22.09 & 37.21 & 430 \\ \text { BLUE-COLLAR } & 17.70 & 15.23 & 2.47 & 19.75 & 44.86 & 243 \\ \text { NOTEMPLOYEO } & 15.96 & 13.62 & 2.82 & 20.66 & 46.95 & 213 \\ \text { OTHER } & 17.54 & 23.68 & 2.63 & 17.54 & 38.60 & 114 \\ \text { AGGREGATE } & 19.10 & 16.50 & 2.40 & 20.70 & 41.30 & 1000\end{array}$


TABLE 32 (continued)

103

TARRANCE \& ASSC. - SAFETY BELT (MOOD) - $\$ 2017$

PQ/PPLE WON'T HAVE ACCUNTS/IN CNTRL OF

AGREE/ST ASREE UNSURE DISAGREE DISAGREE
PONGLY ISTRNGLY

TOTAL

USER CATAGORIES

NOT $\triangle P P L I C A B L E$

FREQ USEPS/CNFRM

$17.6 \dot{8} \quad 14.7 \dot{9}$

$2.2 \dot{5}$

$26.6 \dot{9}$

$38.5 \dot{9}$

1.73

17.32

46.32

$20.65 \quad 20.65$

3.26

11.96

SMTM USER/UNLKLY

19.05

14.29

2.38

20.83

43.48

20.53

23.18

3.31

17.22

43.45

35.76

P65/NHERE DO YOU LIVE

\begin{tabular}{|c|c|c|c|c|c|c|}
\hline $\begin{array}{l}\text { IN A BIG CITY } \\
\text { SUEURBS/BIG CITY } \\
\text { IN A SMALL CITY } \\
\text { TOWN/VILLAGE } \\
\text { IN THE COUNTRY } \\
\text { OK/NO INSWER }\end{array}$ & $\begin{array}{l}19.05 \\
17.07 \\
21.43 \\
18.82 \\
20.47 \\
0.00\end{array}$ & $\begin{array}{l}12.17 \\
21.95 \\
9.82 \\
18.24 \\
19.69 \\
33.33\end{array}$ & $\begin{array}{l}3.70 \\
2.44 \\
2.68 \\
1.18 \\
1.57 \\
0.00\end{array}$ & $\begin{array}{l}23.28 \\
20.56 \\
19.64 \\
23.53 \\
14.96 \\
33.33\end{array}$ & $\begin{array}{l}41.80 \\
37.98 \\
46.43 \\
38.24 \\
43.31 \\
33.33\end{array}$ & $\begin{array}{r}189 \\
287 \\
224 \\
170 \\
127 \\
3\end{array}$ \\
\hline \multicolumn{7}{|c|}{ FREQ-ORV-1 HR ORNKNG } \\
\hline $\begin{array}{l}\text { DAILY TO I/MONTH } \\
\text { SEVEPAL TMS/YR } \\
\text { NEVEP }\end{array}$ & $\begin{array}{l}20.50 \\
22.28 \\
17.63\end{array}$ & $\begin{array}{l}18.00 \\
19.69 \\
14.99\end{array}$ & $\begin{array}{l}2.50 \\
1.55 \\
2.64\end{array}$ & $\begin{array}{l}21.00 \\
17.62 \\
21.58\end{array}$ & $\begin{array}{l}38.00 \\
38.86 \\
43.16\end{array}$ & $\begin{array}{l}200 \\
193 \\
607\end{array}$ \\
\hline$\triangle G G R E G \triangle T E$ & 19.10 & 16.50 & 2.40 & 20.70 & $41 \cdot 30$ & 1000 \\
\hline
\end{tabular}


TABLE 33 - DEMOGRAPHICS BY EFFECT OF BELT ON CONTROL OF CAP.

TARRANCE \& ASSC. - SAFETY BELT (MOOD) - \#20:7

PI8/BELTS/LESS CONTROL OF CAR VS. MORE
THOMPSON MR THOMP UNSURE MR EVANS MR EVANS
ISTRNGLY SON ISTRNGLY

TOTAL

STANDARD FED. ADMNSTRATV REGIONS

$\begin{array}{lrrrrrr}\text { I/NEW ENGLND STS } & 9.26 & 9.26 & 14.81 & 24.07 & 42.59 & 54 \\ \text { IIINW YRK/NIS JRY } & 14.29 & 8.93 & 13.39 & 18.75 & 44.64 & 112 \\ \text { III/MIO ATLANTIC } & 15.24 & 9.52 & 10.48 & 21.90 & 42.86 & 105 \\ \text { IV/SOUTH EAST } & 13.79 & 10.34 & 16.09 & 15.52 & 44.25 & 174 \\ \text { V/GREAT LAKES } & 11.82 & 6.40 & 19.70 & 15.27 & 46.80 & 203 \\ \text { VI/MID SOUTH } & 9.82 & 14.29 & 14.29 & 16.07 & 45.54 & 112 \\ \text { VII/PLAINS } & 15.09 & 7.55 & 13.21 & 16.98 & 47.17 & 53 \\ \text { VIII/NW PLAINS } & 3.13 & 3.13 & 6.25 & 28.13 & 59.38 & 32 \\ \text { IX/WEST COAST } & 7.44 & 7.44 & 14.05 & 26.45 & 44.63 & 121 \\ \text { X/NORTH WEST } & 5.88 & 8.82 & 5.88 & 20.59 & 58.82 & 34 \\ \text { RESPONDENTIS AGE } & & & & & & \\ & & & & & & \\ \text { I8-24 } & 12.69 & 15.67 & 8.21 & 18.66 & 44.78 & 134 \\ 25-44 & 10.53 & 8.84 & 14.74 & 20.42 & 45.47 & 1475 \\ 45 \text { AND OVER } & 12.53 & 6.65 & 16.62 & 17.39 & 46.80 & 391\end{array}$

RESPONDENT'S EOUCATION

$\begin{array}{lrrrrrr}\text { LT HIGH SCHOOL } & 21.43 & 7.14 & 19.64 & 12.50 & 39.29 & 112 \\ \text { HIGH SCHOOL GRAD } & 12.94 & 11.86 & 13.48 & 14.29 & 47.44 & 371 \\ \text { SOME COLLEGE } & 10.37 & 9.26 & 12.96 & 24.81 & 42.59 & 270 \\ \text { COLLEGE GRAD } & 6.48 & 4.86 & 15.79 & 22.67 & 50.20 & 247\end{array}$

RESPONDENT'S SEX

$\begin{array}{lrrrrrr}\text { MALE } & 14.43 & 10.42 & 15.63 & 19.24 & 40.28 & 499 \\ \text { FEMALE/HOME } & 10.29 & 5.76 & 13.58 & 17.28 & 53.09 & 243 \\ \text { FEMALE/EMPLOYEO } & 7.36 & 8.91 & 13.57 & 20.16 & 50.00 & 258 \\ \text { PRIMARY SOURCE OF FAMILY INCOME/C } & & & & \\ \text { SLF-EMPL/WHITE C } & 10.00 & 8.14 & 13.95 & 21.63 & 46.28 & 430 \\ \text { BLUE-COLLAR } & 14.81 & 8.64 & 16.05 & 14.81 & 45.68 & 243 \\ \text { NOT EMPLOYED } & 12.68 & 9.39 & 13.62 & 16.90 & 47.42 & 213 \\ \text { OTHER } & 8.77 & 11.40 & 15.79 & 21.93 & 42.11 & 114 \\ \text { AGGREGATE } & 11.60 & 8.90 & 14.60 & 19.00 & 45.90 & 1000\end{array}$


TABLE 33 (continued)

TARRANCE \& ASSC. - SAFETY BELT (MOOD) - \$2017

P18/BELTS/LESS CONTKOL OF CAR VS. MORE

THOMPSON MR. THOMP UNSURE MR EVANS MR EVANS

ISTRNGLY SON ISTRNGLY

TOTAL

USER CATAGORIES

NOT $\triangle P P L I C A B L E$

FREO USERS/CNFRM

S:MTM USER/LIKELY

SMTM USER/UNLKLY

INFREQ USR/LIKLY

INFREO USR/CNFRM

$\begin{array}{rrrrr}2.57 & 3.22 & 7.40 & 20.90 & 65.92 \\ 6.93 & 7.79 & 13.85 & 19.48 & 51.95 \\ 9.78 & 8.70 & 13.04 & 18.48 & 50.00 \\ 17.26 & 15.07 & 21.43 & 16.67 & 28.57 \\ 31.79 & 13.25 & 19.87 & 15.23 & 19.87\end{array}$

$31 i$

231

92

168

151

PS5/WHERE DO YOU LIVE

IN $\triangle$ BIG CITY

SUBURBS/BIG CITY

IN A SMALL CITY

TOWN/VILLAGE

IN THE COUNTRY

DK/NO ANSWER

$\begin{array}{rrrr}13.76 & 16.88 & 14.81 & 20.11 \\ 8.01 & 10.45 & 13.59 & 22.65 \\ 9.38 & 5.80 & 11.51 & 19.64 \\ 13.53 & 10.59 & 16.47 & 18.24 \\ 18.11 & 11.02 & 16.90 & 8.66 \\ 0.00 & 33.33 & 33.33 & 33.33\end{array}$

FREQ-DRV-1 HP ORNKNG
13.50

7.25

12.36

11.60
12.50
7.25

3.24

3.90
13.00

15.54

14.83

14.60
19.50

25.91

16.64

19.00
44.44
45.30
53.57
41.18
43.31
0.00

189

287

224

170

127

3

AGGPEGATE

11.60 
106

TABLE 34 - DEMOGRAPHICS BY IN CONTROL/CAN PREVENT ACCIDENTS

TARRANCE \& ASSC. - SAFETY BELT (MOOD) - \#2017

PI9/HOW MUCH CONTROL/PREVENT INVLVMNT/A

ALMST TO A LOT OF SOME CON VRY LITT UNSURE

TL CNTRL CONTROL TROL LE CNTRL

TOTAL

STANOARD FED. ADMNSTRATV REGIONS

$\begin{array}{lrllllr}\text { I/NEW ENGLNO STS } & 11.11 & 46.30 & 33.33 & 9.26 & 0.00 & 54 \\ \text { II/NW YRK/NW JRY } & 16.96 & 41.96 & 33.93 & 4.46 & 2.68 & 112 \\ \text { III/MID ATLANTIC } & 12.38 & 44.76 & 36.19 & 3.81 & 2.86 & 105 \\ \text { IV/SOUTH EAST } & 16.67 & 40.23 & 32.76 & 4.60 & 5.75 \ldots . & 174 \\ \text { V/GREAT LAKES } & 14.78 & 38.92 & 38.92 & 5.91 & 1.48 & 203 \\ \text { VI/MID SOUTH } & 16.07 & 42.86 & 35.71 & 5.36 & 0.00 & 112 \\ \text { VII/PLAINS } & 11.32 & 47.17 & 37.74 & 3.77 & 0.00 & 53 \\ \text { VIII/NW PLAINS } & 3.13 & 43.75 & 50.00 & 3.13 & 0.00 & 32 \\ \text { IX/WEST COAST } & 11.57 & 51.24 & 31.40 & 4.13 & 1.05 & 121 \\ \text { X/NORTH WEST } & 5.88 & 50.00 & 41.18 & 2.94 & 0.00 & 34\end{array}$

RESPONOENT'S AGE

$\begin{array}{lllllll}18-24 & 15.67 & 38.81 & 38.06 & 5.22 & 2.24 & 134 \\ 25-44 & 11.37 & 43.58 & 39.16 & 4.84 & 1.05 & 475 \\ 45 \text { ANO OVER } & 16.11 & 44.76 & 30.95 & 4.86 & 3.32 & 391\end{array}$

RESPONDENT'S ECUCATION

$\begin{array}{lllllll}\text { LT HIGH SCHOOL } & 25.00 & 33.04 & 29.46 & 8.04 & 4.46 & 112 \\ \text { HIGH SCHOOL GRAD } & 16.17 & 41.24 & 34.77 & 5.66 & 2.16 & 371 \\ \text { SOME COLLEGE } & 11.85 & 43.70 & 37.78 & 4.07 & 2.59 & 270 \\ \text { COLLEGE GRAD } & 7.29 & 51.01 & 38.06 & 3.24 & 0.40 & 247\end{array}$

RESPONDENT'S SEX

$\begin{array}{lllllll}\text { MALE } & 15.83 & 48.10 & 29.86 & 4.81 & 1.40 & 499 \\ \text { FEMALE/HOME } & 13.17 & 39.51 & 37.45 & 5.76 & 4.12 & 243 \\ \text { FEMALE/EMPLOYED } & 10.47 & 37.98 & 45.74 & 4.26 & 1.55 & 258\end{array}$

PRIMARY SOURCE OF FAMILY INCOME/C

\begin{tabular}{|c|c|c|c|c|c|c|}
\hline $\begin{array}{l}\text { SLF-EMPL/NHITE C } \\
\text { BLUE-COLLAR } \\
\text { NOT EMPLOYED } \\
\text { OTHER }\end{array}$ & $\begin{array}{l}9.30 \\
16.05 \\
18.78 \\
16.67\end{array}$ & $\begin{array}{l}46.51 \\
44.86 \\
37.09 \\
40.35\end{array}$ & $\begin{array}{l}37.21 \\
34.98 \\
33.33 \\
36.84\end{array}$ & $\begin{array}{l}5.58 \\
3.29 \\
5.63 \\
4.39\end{array}$ & $\begin{array}{l}1.40 \\
0.82 \\
5.16 \\
1.75\end{array}$ & $\begin{array}{l}430 \\
243 \\
213 \\
114\end{array}$ \\
\hline & 13.80 & 43.40 & 35.80 & 4.90 & 2.10 & \\
\hline
\end{tabular}


TABLE 34 (continued)

TARRANCE \& ASSC. - SAFETY BELT (MOOO) - \#2017.

P19/HOW MUCH CONTROL/PREVENT INVLVMNT/A

ALMST TO A LOT OF SOME CON VRY LITT UNSURE TL CNTRL CONTROL TROL LE CNTRL

TOTAL

USER CATAGURIES

NOT $\triangle P P L I C A B L E$

FREQ USERS/CNFRM

SMTM USEP. /LIKELY

SHTM USER/UNLKLY

INFREQ USR/LIKLY

INFREQ USR/CNFRM

$\begin{array}{lll}14.47 & 47.59 & 33.76 \\ 10.82 & 46.75 & 34.20 \\ 15.30 & 39.13 & 38.04 \\ 15.48 & 38.10 & 38.69 \\ 14.57 & 41.72 & 35.76\end{array}$

2.89

5.19

5.43

5.36

6.62

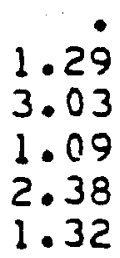

311

231

92

168

151

P65/WHERE DO YOU LIVE

IN A BIE CITY
SUQUPAS/ZIG CITY
IN A SMALL CITY
TOINN /VILLAGE
IN THE COUNTRY
DK/NOARISWER

DK/NO ARSWER

FRED-DPV-1 HR DRNKNG

DAILY TO I/MONTH
SEVEPAL TMS/YP.
NEVER.

AGGREGATE
12.17

13.59

13.84

14.71

14.96

33.33

$\begin{array}{rr}46.03 & 33.86 \\ 42.51 & 37.28 \\ 42.86 & 37.05 \\ 42.94 & 36.47 \\ 44.09 & 32.28 \\ 0.00 & 33.33\end{array}$

$0.00 \quad 33.33$
4.75

5.23

4.02

4.71

6.30

0.00

5.50

2.59

5.44

15.16

13.80
$42.00 \quad 37.00$

49.74

41.85

34.60

$43.40 \quad 35.80$
3.17

1.39

2.23

1.18

2.36

33.33
189 287 224

170

127

200

193

607

$4.90 \quad 2.10$

1000 
TABLE 35 - DEMOGRAPHICS BY PERCEIVED CAUSE OF ACCIDENT

TIR.) ANCE S ASSC. - SAFETY DELT (MOOD) - 2017

PZO/MOST LIKE:Y TO CAUSE AUTO ACEIOENT ONN DRIV A ORUNK OTHRS OR MECHANIC UNAVOBL POOR ROA POOR WEA OKINO AN
NG ERROR ORIVER VNG EFRR L DEFECT BAD LUCK O CNOTNS THER SWER TOTAL

STMOUAOE FE. DOMNSTOATV REJZONS

\begin{tabular}{|c|c|c|c|c|c|c|c|c|c|}
\hline 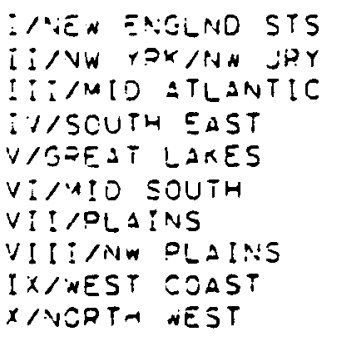 & $\begin{array}{l}.2 .95 \\
2.63 \\
6.67 \\
5.75 \\
3.94 \\
8.93 \\
5.56 \\
3.13 \\
5.79 \\
2.74\end{array}$ & $\begin{array}{l}22.22 \\
20.54 \\
19.10 \\
27.01 \\
19.70 \\
21.43 \\
9.43 \\
19.75 \\
23.14 \\
14.71\end{array}$ & $\begin{array}{l}29.53 \\
43.75 \\
40.00 \\
37.36 \\
40.39 \\
40.17 \\
37.74 \\
25.13 \\
47.59 \\
50.00\end{array}$ & $\begin{array}{l}3.70 \\
0.00 \\
0.95 \\
6.32 \\
1.48 \\
2.68 \\
7.35 \\
0.00 \\
0.83 \\
2.74\end{array}$ & $\begin{array}{l}14.81 \\
8.93 \\
3.81 \\
7.47 \\
5.91 \\
5.36 \\
9.43 \\
3.13 \\
9.09 \\
5.88\end{array}$ & $\begin{array}{l}3.70 \\
5.36 \\
8.57 \\
3.45 \\
7.39 \\
4.46 \\
7.55 \\
9.38 \\
2.48 \\
5.88\end{array}$ & $\begin{array}{l}11.11 \\
13.39 \\
17.14 \\
9.20 \\
15.75 \\
11.61 \\
18.87 \\
34.38 \\
7.44 \\
8.32\end{array}$ & $\begin{array}{l}1.85 \\
5.36 \\
4.76 \\
3.45 \\
4.43 \\
5.36 \\
3.77 \\
3.13 \\
1.65 \\
8.32\end{array}$ & $\begin{array}{r}54 \\
112 \\
105 \\
174 \\
203 \\
112 \\
53 \\
32 \\
121 \\
34\end{array}$ \\
\hline \multicolumn{10}{|l|}{ aESPDNUEMTT IS AGE } \\
\hline $\begin{array}{l}: \equiv-? 4 \\
Z \equiv-44 \\
i 5 \text { AND CVEK }\end{array}$ & $\begin{array}{l}5.72 \\
5.89 \\
5.12\end{array}$ & $\begin{array}{l}17.91 \\
15.34 \\
25.85\end{array}$ & $\begin{array}{l}35.82 \\
46.32 \\
35.04\end{array}$ & $\begin{array}{l}2.24 \\
2.75 \\
2.30\end{array}$ & $\begin{array}{r}14.93 \\
6.53 \\
5.37\end{array}$ & $\begin{array}{l}6.72 \\
5.39 \\
4.50\end{array}$ & $\begin{array}{l}14.18 \\
11.79 \\
15.35\end{array}$ & $\begin{array}{l}1.49 \\
3.79 \\
5.37\end{array}$ & $\begin{array}{l}134 \\
475 \\
391\end{array}$ \\
\hline \multicolumn{10}{|c|}{ ZESPONIENTIS EOUCATIOP } \\
\hline $\begin{array}{l}\text { T -TGN SCHDOL } \\
\text { IGH SCHOOL GRAD } \\
\text { SOME COLLEGE } \\
\text { SJLLEUE ORAO }\end{array}$ & $\begin{array}{l}5.25 \\
3.77 \\
5.73 \\
3.10\end{array}$ & $\begin{array}{l}29.57 \\
20.22 \\
20.76 \\
13.62\end{array}$ & $\begin{array}{r}21.43 \\
37.74 \\
45.56 \\
-7.77\end{array}$ & $\begin{array}{l}3.57 \\
3.23 \\
2.96 \\
0.81\end{array}$ & $\begin{array}{l}8.04 \\
0.63 \\
9.26 \\
2.43\end{array}$ & $\begin{array}{l}7.14 \\
5.93 \\
3.70 \\
6.07\end{array}$ & $\begin{array}{r}21.43 \\
16.17 \\
8.52 \\
11.34\end{array}$ & $\begin{array}{l}3.57 \\
4.31 \\
3.33 \\
4.86\end{array}$ & $\begin{array}{l}1: 2 \\
371 \\
270 \\
247\end{array}$ \\
\hline \multicolumn{10}{|l|}{ בEZPONDENTIS SEX } \\
\hline $\begin{array}{l}\text { MLLE } \\
F \equiv M A L E / H O M E \\
\text { FEMALE/EMPLOYED }\end{array}$ & $\begin{array}{l}6.01 \\
5.35 \\
5.23\end{array}$ & $\begin{array}{l}19.84 \\
24.28 \\
13.77\end{array}$ & $\begin{array}{l}43.89 \\
37.04 \\
37.21\end{array}$ & $\begin{array}{l}3.41 \\
1.23 \\
2.33\end{array}$ & $\begin{array}{r}7.01 \\
4.53 \\
10.08\end{array}$ & $\begin{array}{l}5.21 \\
5.76 \\
5.31\end{array}$ & $\begin{array}{l}12.42 \\
16.05 \\
13.18\end{array}$ & $\begin{array}{l}2.20 \\
5.76 \\
5.20\end{array}$ & $\begin{array}{l}499 \\
243 \\
258\end{array}$ \\
\hline DOIMART SOUPCE OF & FSMILY IA & $M E / C$ & $\therefore \quad \vdots$ & & $\cdot$ & 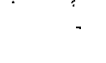 & & & - \\
\hline $\begin{array}{l}\text { SLE EMPL/WHTTE } \\
\text { ZLUE-CJULAR } \\
\text { NOT EMOLOYEO } \\
\text { DTHE? }\end{array}$ & $\begin{array}{l}5.28 \\
5.35 \\
5.15 \\
5.25\end{array}$ & $\begin{array}{l}13.37 \\
20.58 \\
25.35 \\
22.81\end{array}$ & $\begin{array}{l}48.14 \\
30.21 \\
32.39 \\
35.96\end{array}$ & $\begin{array}{l}1.16 \\
3.70 \\
3.76 \\
3.51\end{array}$ & $\begin{array}{l}7.44 \\
9.05 \\
5.10 \\
4.39\end{array}$ & $\begin{array}{l}4.65 \\
? .41 \\
3.76 \\
7.39\end{array}$ & $\begin{array}{l}9.07 \\
14.40 \\
20.19 \\
15.79\end{array}$ & $\begin{array}{l}4.88 \\
3.29 \\
3.29 \\
4.39\end{array}$ & $\begin{array}{l}430 \\
243 \\
213 \\
114\end{array}$ \\
\hline AGGF̈EGATE & 5.70 & 20.90 & 40.50 & 2.60 & 7.20 & 5.50 & 13.50 & 4.10 & 1000 \\
\hline
\end{tabular}


TABLE 35 (continued)

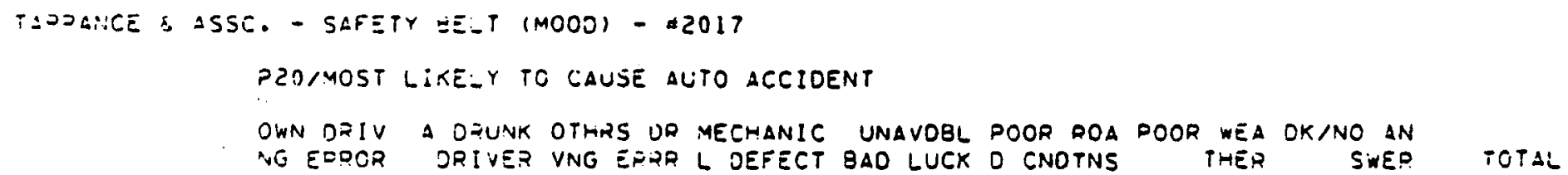

$5.7 \dot{5} \quad 26.4 \dot{4} \quad 4 j .7 j$

$3.46 \quad 17.91 \quad 43.29$

$3.25 \quad 13.43$

$0.33 \quad 13.45$

41.30

36.70

$7.29 \quad 17.22 \quad 35.76$

$\begin{array}{ll}1.9 j & 5.75 \\ 2.15 & 7.36 \\ 2.17 & 6.52 \\ 2.48 & 7.14 \\ 3.97 & 7.28\end{array}$

3.54

0.49

4.35

7.14

5.62

9.32

13.42

18.40

14.88

17.22

3.70

2.65

42.06

5.29 .22 .22

3.27

5.70

50.71

5.

3.15

0.00

17.06

25.95

0.00

44.25

$30: 34$

33.07

00.67

1.39

3.13

7.41

7.32

a. 04

2.96

3.94

8.32

3.15

0.00

3.83

5.36

11.11

13.57

13.34

10.59

19.67

7.09

33.33

0.00

3.00

$7.50 \quad i \div .00$

5.74

14.51

2. 73

$25.2 \mathrm{i}$

$+7.50$

43.52

4.50

36.57

3.11

1.81

5.70

5.50

6.22
5.27

5.70

20.70

$4 v .50$

2.60

7.20

5.50

9.00

15.06

16.17

2.00

4.78

200

193

507

1000 
When the issue of control is addressed in terms of preventing an auto accident, 93\% indicate that they have at least "some control" in preventing their involvement in an accident. Sixty-eight percent (68\%) of the same group reports that if they were involved in an auto accident, the likely cause would be "the other guy".

This combination of responses strongly suggests that the average driver feels that they are in control and if an accident does occur, it's because of "the other guy". Therefore, the safety belt message must be attached to "the other guy" since they cause accidents.

The question can be raised as to whether or not this perception of "the other guy" is an accurate one or not. Data from the 1980 Accident Reporting System clearly indicates that the public perception is correct. Table 36 provides the data on passenger car fatalities by seating position.

If one assumes that at least one-third of the drivers, or about 5,985 drivers, could not have avoided the crash and that none of the passengers could have avoided the crash, then about 14,443 occupants out of 26,411 occupants with a known seating position or about $55 \%$ of passenger car fatalities were, indeed, victims of the "other guy". 
TABLE 36

PASSENGER CAR FATALITIES BY SEATING POSITION

Position

Driver

Passenger

Total known

Unknown

TOTAL

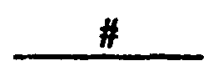

17,953

8,458

26,411

1,022

27,433
\% Total Known Position

$68 \%$

$32 \%$

$100 \%$ 


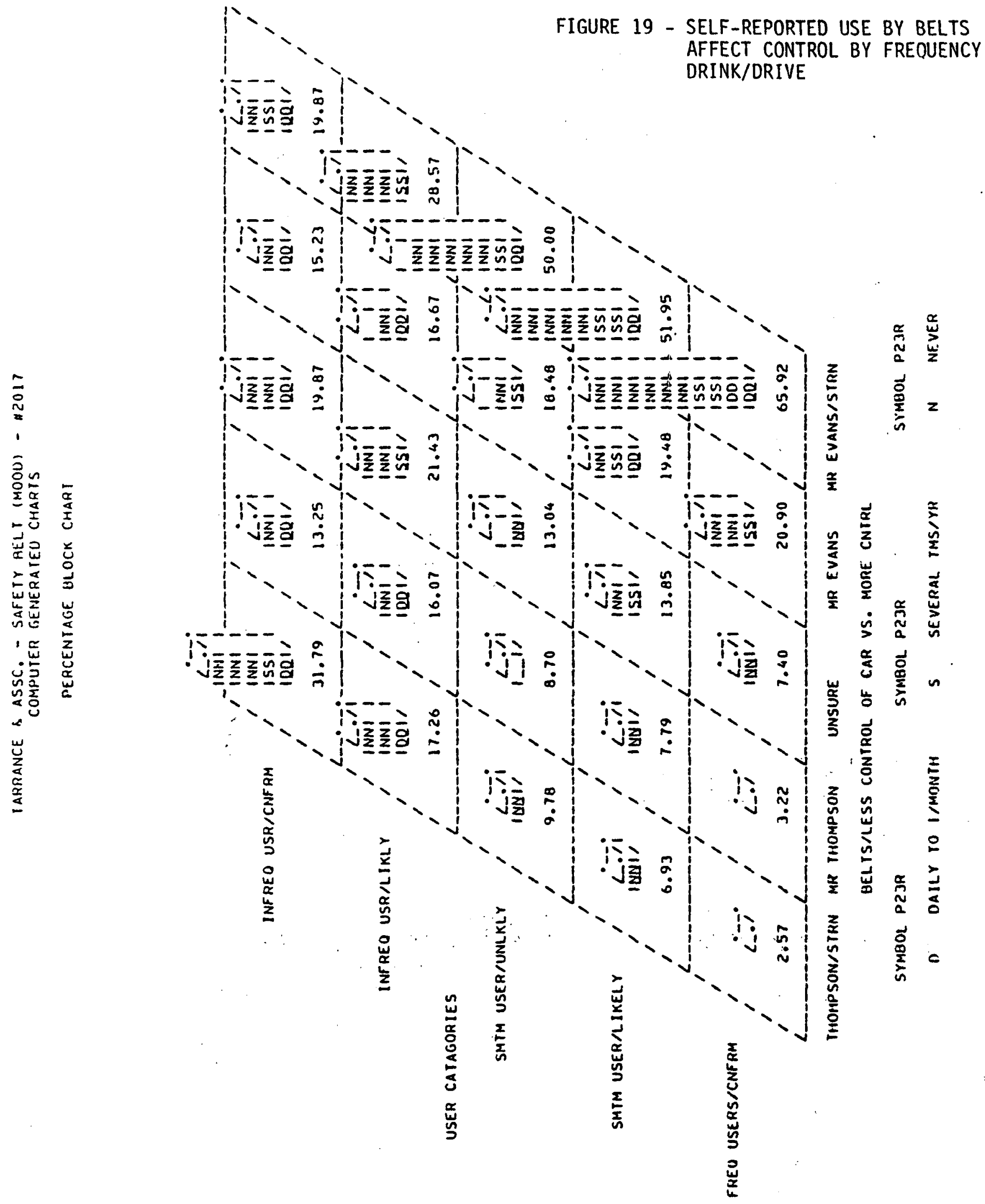


113
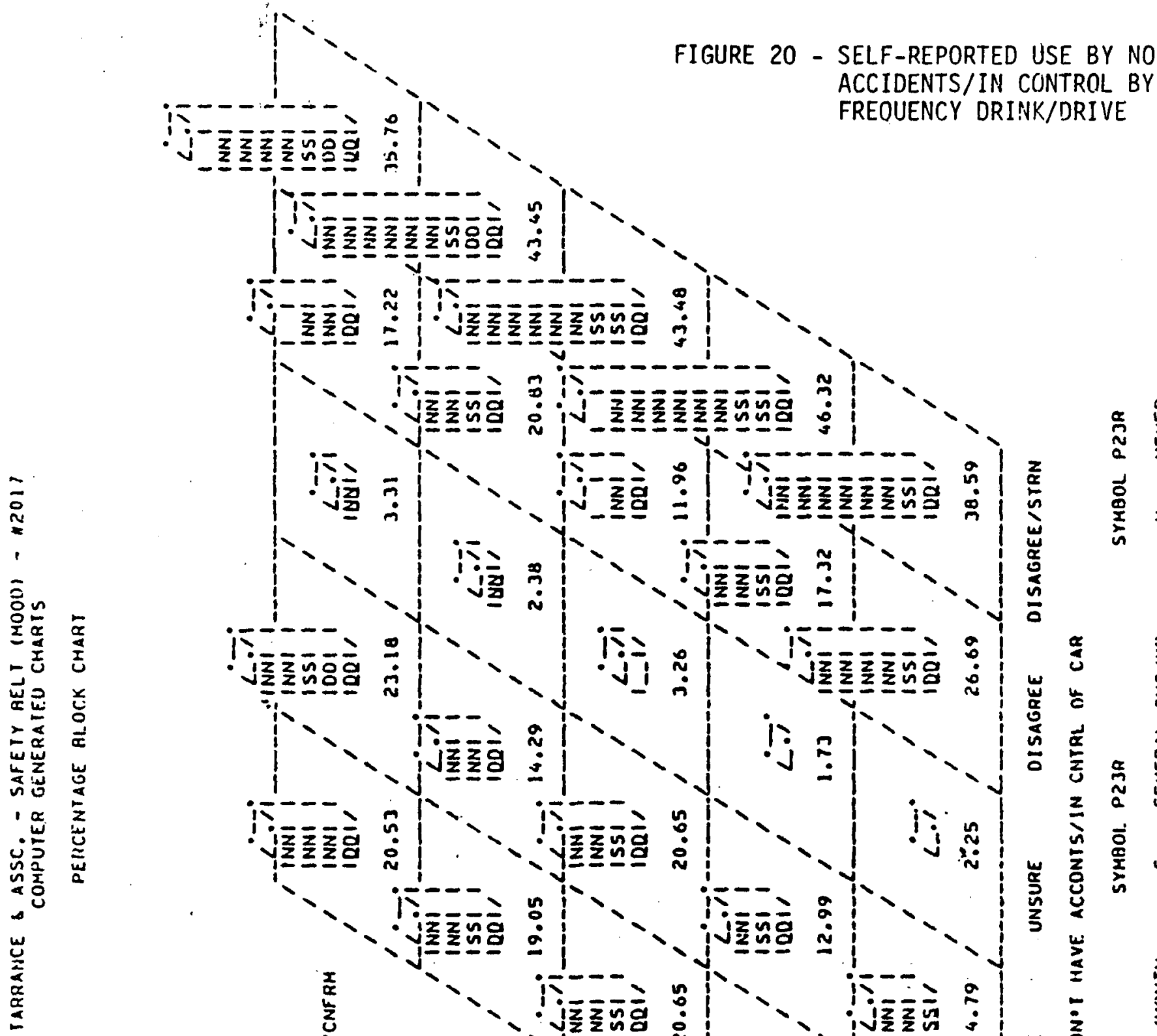
114

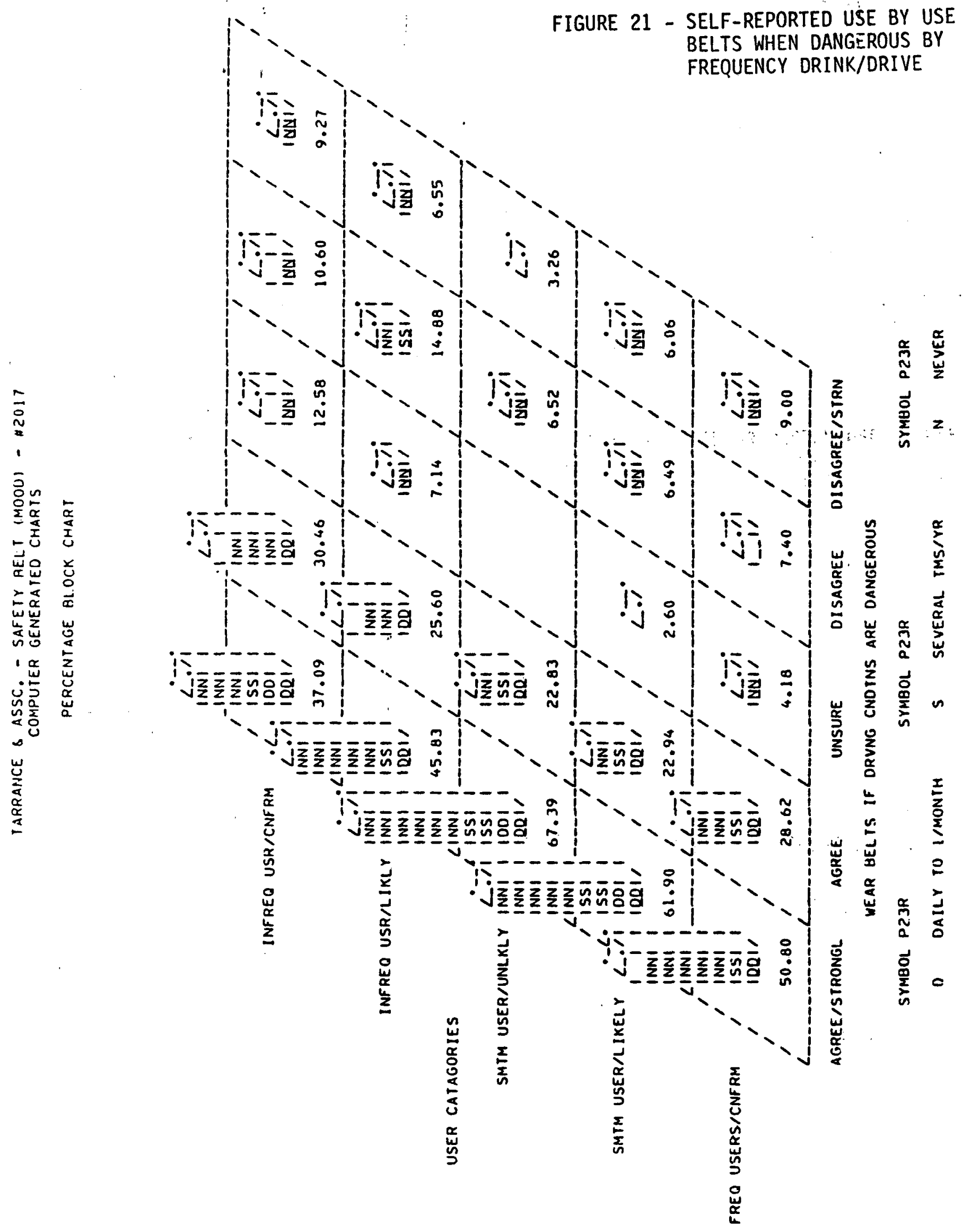




\section{THE PRODUCT'S IMAGE}

This project provided an interesting and useful opportunity to be able to compare the same individuals' responses to questions relating to both the drunk driving issue and the safety belt issue. The initial focus group results clearly established one very important difference between the two programs. The groups immediately established that the drunk driving problem was the other guy's problem. That is, they were able to externalize the issue and therefore their reasons for supporting the program. On the other hand, the safety belt program had been perceived as a program which tended to internalize the issue to either the driver and/ or the driver's car. This translated into "the program is telling me that I'm not a guod driver or my car is not safe". Both of these perceptions are considered negative by the respondents and don't fit into their self-perception of themselves or their cars. As a result, a very strong defense mechanism is developed which translates into "It won't happen to me". Once the individual has developed the defense mechanism of "it won't happen to me", there is no reasen to attend to any message that deals with the issue in that way, since he/she has a ready answer -- "It won't happen to me."

When, however, the issue of safety belt use is addressed as a preventive health measure, or a way of promoting "wellness", the use of the safety belt is perceived in a more positive fashion. By moving the safety belt into either the "wellness area" or into the "preventive health" area, the reason for using the belt is externalized and the individual is not forced to deny its utility.

A number of questions in the survey addressed the issue of the relation between safety belt use and other preventive health practices and issues. These are presented in Tables 37 to 42.

The data is very consistent across all of the survey items in that the individuals who use belts often are more likely to engage in the preventive health measure or agree with the statement than are the non-belt 
116

TABLE 37 - DEMOGRAPHICS BY BELTS ARE LIKE TOOTHBRUSH

TARRANCE \& ASSC. - SAFETY BELT (MOOD) - \#2017

P8/WEARING BELTS/LIKE BRUSHING TEETH

AGREE/ST AGREE UNSURE DISAGREE DISAGREE
PONGLY
/STRNGLY TOTAL

STANDARO FED. AOMNSTRATV REGIONS

$\begin{array}{llllrrr}\text { I/NEW ENGLNO STS } & 42.59 & 29.63 & 1.85 & 12.96 & 12.96 & 54 \\ \text { II/NW YRK/NW JRY } & 52.68 & 28.57 & 0.89 & 5.36 & 12.50 & 112 \\ \text { III/MID ATLANTIC } & 52.38 & 29.52 & 0.95 & 6.67 & 10.48 & 105 \\ \text { IV/SOUTH EAST } & 48.28 & 31.61 & 2.87 & 9.77 & 7.47 & 174 \\ \text { V/GREAT LAKES } & 55.67 & 25.11 & 2.46 & 7.88 & 7.88 & 203 \\ \text { VI/MID SOUTH } & 58.93 & 23.21 & 1.79 & 13.39 & 2.68 & 112 \\ \text { VII/PLAINS } & 52.83 & 24.53 & 1.89 & 11.32 & 9.43 & 53 \\ \text { VIII/NW PLAINS } & 62.50 & 34.38 & 0.00 & 0.00 & 3.13 & 32 \\ \text { IX/WEST COAST } & 54.55 & 28.93 & 4.96 & 3.31 & 8.26 & 121 \\ \text { X/NORTH WEST } & 44.12 & 38.24 & 8.82 & 5.88 & 2.94 & 34\end{array}$

RESPONDENT'S AGE

$\begin{array}{lrrrrrr}18-24 & 50.00 & 31.34 & 0.75 & 12.59 & 5.22 & 134 \\ 25-44 & 51.79 & 30.32 & 2.11 & 8.00 & 7.79 & 475 \\ 45 \text { AND OVER } & 55.24 & 25.32 & 3.58 & 6.39 & 9.46 & 391\end{array}$

RESPONDENT'S EDUCATION.

$\begin{array}{lrlrrrr}\text { LT HIGH SCHOOL } & 51.79 & 25.00 & 0.25 & 5.36 & 11.61 & 112 \\ \text { HIGH SCHOOL GRAD } & 52.29 & 27.76 & 2.43 & 8.63 & 8.89 & 371 \\ \text { SOME COLLEGE } & 48.89 & 28.52 & 2.59 & 10.74 & 9.26 & 270 \\ \text { COLLEGE GPAD } & 58.70 & 31.17 & 0.81 & 5.26 & 4.05 & 247\end{array}$

RESPONDENT'S SEX

$\begin{array}{lllllll}\text { MALE } & 48.90 & 29.66 & 2.81 & 9.42 & 9.22 & 499 \\ \text { FEMALE/HOME } & 57.20 & 26.34 & 2.47 & 6.58 & 7.41 & 243 \\ \text { FEMALE/EMPLOYED } & 56.59 & 28.29 & 1.94 & 6.59 & 6.59 & 258\end{array}$

PRIMARY SOURCE OF FAMILY INCOME/C

$\begin{array}{lrrrrrr}\text { SLF-EMPL/WHITE C } & 52.33 & 28.60 & 2.33 & 8.84 & 7.91 & 430 \\ \text { BLUE-COLLAR } & 54.73 & 27.98 & 1.65 & 8.23 & 7.41 & 243 \\ \text { NOTEMPLOYED } & 53.05 & 26.76 & 3.76 & 4.69 & 11.74 & 213 \\ \text { OTHER } & 50.88 & 32.46 & 2.63 & 10.53 & 3.51 & 114 \\ \text { AGGREGATE } & 52.90 & 28.50 & 2.50 & 8.00 & 8.10 & 1000\end{array}$


TABLE 37 (continued)

TARRANCE \& ASSC. - SAFETY BELT (MOOD) - $\# 2017$

P8/WEARING BELTS/LIKE BRUSHING TEETH

AGREE/ST AGREE UNSURE DISAGREE DISAGREE
RONGLY ISTRNGLY

TOTAL

USER CATAGORIES

NOT $\triangle P P L I C A B L E$

FREO USERS/CNFRM

SMTM USER/LIKELY

$70.7 \dot{4}$

$25.0 \dot{8}$

0.96

29.00

61.04

25.00

SMTM USER/UNLKLY

57.61

34.52

1.30

4.35

37.50

4.17

25.17

1.99

1.29
3.46
7.61
13.10
22.52

1.93

5.19

5.43

25.83

10.71

24.50

311

231

92

168

151

P65/WHERE DO YOU LIVE

\section{A BIG CITY \\ SUBURBS/BIG CITY \\ IN A SMALL CITY \\ TOWN/VILLAGE \\ IN THE COUNTRY}

DK/NO ANSWER

$\begin{array}{lr}58.20 & 21.69 \\ 47.74 & 35.54 \\ 57.59 & 25.45 \\ 52.35 & 27.65 \\ 49.61 & 29.92 \\ 33.33 & 0.00\end{array}$

2.12

0.35

3.13

5.29

3.15

$0.00^{\circ}$
8.99

8.04

5.88

7.87

66.67

8.99

189

287

224

170

127

9.45

0.00

3

FREQ-DRV-1 HR DRNKNG

DAILY TO I/MONTH

SEVERAL TMS/YR

NEVER

AGGREGATE
44.50

30.50

29.53

27.51

$52.90 \quad 25.50$
2.50

1.55

2.80

2.50
9.50
10.36

10.36

6.75

8.00
13.00

3.63

7.91

8.10
200

193

607

1000 
118

TABLE 38 - DEMOGRAPHICS BY DEGREE OF INCONVENIENCE

TARRANCE \& ASSC. - SAFETY BELT (MOOD) - \#2017

\section{PI6/MAJOR INCONVENIENCE VS. SLIGHT INCN}

MR JHNSN MR JOHNS UNSURE MR DAVIS MR DAVIS

ISTRNGLY ON ISTRNGLY

TOTAL

STANOARO FED. ADMNSTRATV REGIONS

I /NEW ENGLND STS

I.I/NW YRK/NW JRY

III/MIO ATLANTIC

IV/SOUTH EAST

VIGREAT LAKES

VI/MID SOUTH

VII/PLAINS

VIII/NIN PLAINS

IX/WEST COAST

$X /$ NORTH WEST

$\begin{array}{rr}20.37 & 16.67 \\ 12.50 & 10.71 \\ 14.29 & 15.24 \\ 12.07 & 8.05 \\ 11.33 & 11.82 \\ 17.86 & 18.75 \\ 18.87 & 13.21 \\ 15.63 & 15.63 \\ 6.61 & 11.57 \\ 2.94 & 17.65\end{array}$

RESPONOENT'S AGE

$18-24$

$25-44$

45 AND OVER

$\begin{array}{ll}12.69 & 11.19 \\ 14.53 & 15.16 \\ 10.74 & 10.49\end{array}$

0.75

4.84

6.39

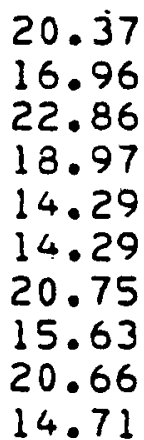

14.71
40.74

54.46

46.67

53.45

54.68

44.64

43.40

53.13

58.68

58.82
54

112

105

174

203

112

53

32

121

34

RESPONDENT'S EDUCATION

\section{LT HIGH SCHOOL \\ HIGH SCHOOL GRAD \\ SOME COLLEGE \\ COLLEGE GRAD \\ RESPONDENT'S SEX}

$\begin{array}{ll}17.86 & 7.14 \\ 13.75 & 12.13 \\ 10.74 & 14.81 \\ 11.34 & 14.17\end{array}$

14.29

4.85

3.33

12.50

17.16

16.63

58.21

134

48.84

52.94

$\begin{array}{lllllll}\text { MALE } & 14.03 & 15.83 & 4.81 & 17.84 & 47.49 & 499 \\ \text { FEMALE/HOME } & 10.70 & 7.41 & 6.17 & 15.46 & 59.26 & 243 \\ \text { FEMALE/EMPLOYED } & 12.40 & 12.02 & 3.88 & 18.99 & 52.71 & 258\end{array}$

PRIMARY SOURCE OF FAMILY INCOME/C.

\begin{tabular}{|c|c|c|c|c|c|c|}
\hline $\begin{array}{l}\text { SLF-EMPL/WHITE C } \\
\text { BLUE-COLLAR } \\
\text { NOT EMPLOYED } \\
\text { OTHER }\end{array}$ & $\begin{array}{r}12.09 \\
16.46 \\
9.39 \\
14.04\end{array}$ & $\begin{array}{r}13.02 \\
14.40 \\
3.92 \\
15.79\end{array}$ & $\begin{array}{l}3.49 \\
4.12 \\
7.98 \\
6.14\end{array}$ & $\begin{array}{l}19.53 \\
14.81 \\
18.31 \\
16.67\end{array}$ & $\begin{array}{l}51.86 \\
50.21 \\
55.40 \\
47.37\end{array}$ & $\begin{array}{l}430 \\
243 \\
213 \\
114\end{array}$ \\
\hline AGGREGATE & 12.80 & 12.80 & 4.90 & 17.80 & 51.70 & 1000 \\
\hline
\end{tabular}


TABLE 38 (continued)

119

TARRANCE \& ASSC. - SAFETY EELT (MOOD) - \#2017

PI6/MAJOR INCONVENIENCE VS. SLIGHT INCN

MR JHNSN MR JOHNS UNSURE MR DAVIS MR DAVIS

ISTRNGLY ON ISTRNGLY

TOTAL

USER CATAGORIES

NOT $\triangle P P L I C A B L E$

FREQ USERS/CNFRM

$\begin{array}{rr}2.89 & 2.57 \\ 10.39 & 11.69 \\ 13.04 & 15.30 \\ 19.05 & 21.43 \\ 29.80 & 23.84\end{array}$

$0.6 \dot{4}$

$17.3 \dot{6}$

2.16

21.65

6.52

17.39

6.55

14.29

13.25

11.92

76.53

54.11

46.74

38.69

INFREQ USR/CNFRM

PO5/WHERE DO YOU LIVE

IN A BIG CITY
SUEURBS/BIG CITY
IN A SIALL CITY
TOWNIVILLAGE
IN THE COUNTRY
DK/NO ANSWER

DKINO ANSWER

$\begin{array}{rr}12.17 & 11.64 \\ 15.33 & 11.15 \\ 9.38 & 15.96 \\ 14.12 & 11.18 \\ 12.60 & 12.60 \\ 0.00 & 33.33\end{array}$

$\begin{array}{rr}12.17 & 11.64 \\ 15.33 & 11.15 \\ 9.38 & 15.96 \\ 14.12 & 11.18 \\ 12.60 & 12.60 \\ 0.00 & 33.33\end{array}$

5.82

4.53

3.57

2.35

$\rightarrow .45$

33.33
16.93

19.86

16.96

21.18

11.81

0.00
53.44 49.13

53.13

51.18

53.54

33.33
$31 i$

231

92

168

151

FREO-DRV-1 HR DRNKNG

$\begin{array}{llllll}\text { DAILY TO 1/MONTH } & 16.00 & 18.50 & 2.00 & 15.50 & 48.00 \\ \text { SEVERAL TMS/YR } & 12.95 & 12.95 & 2.07 & 20.21 & 51.81 \\ \text { NEVER } & 11.70 & 10.87 & 6.75 & 17.79 & 52.88 \\ \text { AGGPEGATE } & 12.80 & 12.80 & 4.90 & 17.80 & 51.70\end{array}$

200

193 607

1000 
TABLE 39 - DEMOGRAPHICS BY SMOKING HABITS

TARRANCE \& ASSC. - SAFETY BELT (MOOD) - \#2017

P58/HOW CIGARETTES DO YOU SMOKE A DAY
DON T SM LESS THN 1/2-LSS I PACK O
OKE I/2 FCK THN PACK R MORE TOTAL

STANDARO FED. ADMNSTRATV REGIONS

I/NEW ENGLNO STS

II NWW YRK/NW JRY

III/MIO ATLANTIC

IV/SOUTH EAST

VIGREAT LAKES

VI/MIO SOUTH

VII/PLAINS

VIII/NW PLAINS

IX/WEST COAST

$X / N O R T H$ WEST

$\begin{array}{ll}68.52 & 3.70 \\ 73.21 & 3.57 \\ 67.62 & 5.71 \\ 68.97 & 9.20 \\ 69.95 & 8.87 \\ 73.21 & 4.46 \\ 77.36 & 5.66 \\ 65.63 & 3.13 \\ 74.38 & 5.26 \\ 76.47 & 0.00\end{array}$

RESPONDENT'S $\triangle G E$

$\begin{array}{llrrrr}18-24 & 79.85 & 3.73 & 7.96 & 7.46 & 134 \\ 25-44 & 67.58 & 7.79 & 5.26 & 19.37 & 475 \\ 45 \text { AND OVER } & 72.63 & 5.88 & 6.65 & 14.83 & 391\end{array}$

RESPONDENT'S EDUCATION

$\begin{array}{llllll}\text { LT HIGH SCHOOL } & 64.29 & 8.04 & 8.93 & 18.75 & 112 \\ \text { HIGH SCHDOL GRAO } & 67.65 & 7.28 & 6.47 & 18.60 & 371 \\ \text { SOME COLLEGE } & 72.59 & 5.56 & 5.93 & 15.93 & 270 \\ \text { COLLEGE GRAD } & 78.14 & 5.67 & 5.26 & 10.93 & 247\end{array}$

RESPONDENTIS SEX

$\begin{array}{llllll}\text { MALE } & 70.34 & 6.81 & 5.41 & 17.43 & 499 \\ \text { FEMALE/HOME } & 71.19 & 6.17 & 6.17 & 16.46 & 243 \\ \text { FEMALE/EMPLOYED } & 72.87 & 6.20 & 8.14 & 12.79 & 258\end{array}$

PRIMARY SOURCE OF FAMILY INCOME/C

$\begin{array}{lllllr}\text { SLF-EMPL/WHITE C } & 73.26 & 4.88 & 6.51 & 15.35 & 430 \\ \text { BLUE-COLLAR } & 65.84 & 8.23 & 5.76 & 20.16 & 243 \\ \text { NOT EMPLOYED } & 71.36 & 7.98 & 6.10 & 14.55 & 213 \\ \text { OTHER } & 74.56 & 6.14 & 7.02 & 12.28 & 114 \\ & & & & & \\ \text { AGGREGATE } & 71.20 & 6.50 & 6.30 & 16.00 & 1000\end{array}$


TABLE 39 (continued)

TARRANCE \& ASSC. - SAFETY BELT (MOOD) - \#2017

$$
\begin{aligned}
& \text { PSB/HOW CIGARETTES DO YOU SMOKE A DAY } \\
& \text { DON'T SM IESS THN 1/2-LSS I FACK O } \\
& \text { OKE I/2 PCK THN PACK R MORE TOTAL }
\end{aligned}
$$

USER CATAGORIES

$\begin{array}{llllll}\text { NOT APDLICABLE } & & & & \\ \text { FREQ USERS/CNFRM } & 74.60 & 6.75 & 5.14 & 13.50 & 311 \\ \text { SMTM USER/LIKELY } & 73.59 & 7.79 & 6.49 & 12.12 & 231 \\ \text { SMTM USEP/UNLKLY } & 68.48 & 7.61 & 7.61 & 16.30 & 92 \\ \text { INFRED USR/LIKLY } & 66.67 & 5.36 & 7.74 & 20.24 & 168 \\ \text { INFREQ USR/CNFRM } & 64.90 & 3.31 & 7.95 & 23.84 & 151\end{array}$

PS5/WHERE DO YOU LIVE

$\begin{array}{lllllr}\text { IN A BIG CITY } & 75.13 & 5.29 & 4.76 & 14.81 & 189 \\ \text { SUBURBS/BIG CITY } & 71.78 & 8.01 & 7.67 & 12.54 & 287 \\ \text { IN A SMALL CITY } & 72.32 & 5.80 & 5.80 & 16.07 & 224 \\ \text { TOWN/VILLAGE } & 64.71 & 7.65 & 7.65 & 20.00 & 170 \\ \text { IN THE COUNTRY } & 70.87 & 4.72 & 4.72 & 19.69 & 127 \\ \text { OKINO ANSWER } & 66.67 & 0.00 & 0.00 & 33.33 & 3 \\ \text { FREO-ORV-1 HR DRNKNG } & & & & & \\ \text { OAILY TO I/MONTH } & 52.50 & 9.00 & 0.50 & 22.00 & 200 \\ \text { SEVERAL TMS/YR } & 72.02 & 5.18 & 7.77 & 15.03 & 193 \\ \text { NEVER. } & 73.81 & 6.10 & 5.77 & 14.33 & 607 \\ \text { AGGREGATE } & & & & & \\ & 71.20 & 5.50 & 6.30 & 16.00 & 1000\end{array}$


122

TABLE 40 - DEMOGRAPHICS BY FREOOENCY VISIT DENTIST

TARRANCE \& ASSC. - SAFETY BELT (MOOD) - \#2017

PS9/HOW OFTEN DO YOU VISIT THE DENTIST

EVERY 6 EVERY YE EVRY 2 Y SOMTHNG OK/NO AN MONTHS AR RS/MORE BTHRS ME SWER

TOTAL

STANDARD FED. ADMNSTRATV REGIONS

I/NEW ENGLND STS

II/NW YRK/NW JRY

III/MIO ATLANTIC

IV/SOUTH EAST

$V / G R E A T$ LAKES

VI/MIO SOUTH

VII / PLAINS

VIII/NW PLAINS

IX/WEST COAST

$X / N O R T H$ WEST

$\begin{array}{ll}53.70 & 20.37 \\ 42.86 & 32.14 \\ 41.90 & 28.57 \\ 35.63 & 31.03 \\ 40.89 & 30.05 \\ 36.61 & 33.93 \\ 39.62 & 22.64 \\ 37.50 & 34.38 \\ 49.59 & 26.45 \\ 23.53 & 52.94\end{array}$

RESPONOENT'S AGE
$18-24$
$25-44$
45 AND OVER
RESPONDENTIS EDUCATION

LT HIGH SCHOOL

HIGH SCHOOL GRAD

SOME COLLEGE

COLLEGE GRAO

$\begin{array}{ll}20.54 & 20.54 \\ 42.59 & 28.57 \\ 37.41 & 34.81 \\ 51.01 & 32.39\end{array}$

RESPONDENT'S SEX

$\begin{array}{lllllll}\text { MALE } & 39.68 & 30.06 & 8.22 & 19.84 & 2.20 & 499 \\ \text { FEMALE/HOME } & 37.86 & 28.81 & 8.64 & 20.58 & 4.12 & 243 \\ \text { FEMALE/EMPLOYEO } & 45.74 & 32.17 & 5.43 & 15.50 & 1.16 & 258\end{array}$

54

112

105

174

203

112

53

32

121

34

134

475

391

112

371

270

247

PRIMARY SOURCE OF FAMILY INCOME/C

\begin{tabular}{|c|c|c|c|c|c|c|}
\hline $\begin{array}{l}\text { SLF-EMPL/WHITE C } \\
\text { BLUE-COLLAR } \\
\text { NOT EMPLOYED } \\
\text { OTHER }\end{array}$ & $\begin{array}{l}47.44 \\
36.21 \\
34.74 \\
36.84\end{array}$ & $\begin{array}{l}31.63 \\
30.04 \\
25.76 \\
32.46\end{array}$ & $\begin{array}{r}6.28 \\
9.05 \\
7.04 \\
10.53\end{array}$ & $\begin{array}{l}13.02 \\
22.63 \\
25.82 \\
20.18\end{array}$ & $\begin{array}{l}1.63 \\
2.06 \\
5.63 \\
0.00\end{array}$ & $\begin{array}{l}430 \\
243 \\
213 \\
114\end{array}$ \\
\hline$\triangle G G R E G \triangle T E$ & 40.80 & 30.30 & 7.60 & 18.90 & 2.40 & 1000 \\
\hline
\end{tabular}


TABLE 40 (continued)

123

TARRANCE \& ASSC. - SAFETY BELT (MOOD) - \#2017

PS9/HOW OFTEN DO YOU VISIT THE OENTIST

EVERY $S$ EVERY YE EVRY 2 Y SOMTHNG DK/NO AN MONTHS AR RS/MORE BTHRS ME SWER

TOTAL

USER CATAGCRIES

NOT $\triangle P D L I C A B L E$

FREO USERS/CNFRM

SMTM USER/LIKELY

$49.8 \dot{4} \quad 25.6 \dot{9}$

$44.16 \quad 33.33$

$32.61 \quad 31.52$

$33.33 \quad 35.12$

33.77

INFREO USR/CNFRM

PES/WHERE DO YOU LIVE
IN $A$ BIG CITY

SUEURBS/BIG CITY

IN A SMALL CITY

TOWN/VILLAGE

IN THE COUNTRY

OK/NO ANSWEP $\begin{array}{rr}42.86 & 29.04 \\ 42.86 & 32.06 \\ 38.84 & 29.02 \\ 41.18 & 33.53 \\ 37.01 & 27.56 \\ 0.00 & 33.33\end{array}$
9.32

6.49

5.43

7.14

7.28
$11.5 \dot{8}$

13.85

27.17

23.21

29.14
2.57

2.16

3.25

1.19

1.32
$31 i$

231

92

168

151
FREQ-DRV-I HR DRIIKNG
DAILY TO I/MONTH

SEVERAL TMS/YR

NEVEP.

AGGREGATE
$38.50 \quad 35.00$

$45.08 \quad 33.58$

40.20

27.68

$40.80 \quad 30.30$
5.88

7.67

7.38

5.88

7.87

0.00
19.58

16.03

19.64

16.47

25.20

66.67
2.65

1.39

3.13

2.94

2.36

0.00
189

287

224

170

127

3 
TABLE 41 - DEMOGRAPHICS BY EXERCISE HABITS

TARRANCE \& ASSC. - SAFETY BELT (MOOD) - \#2017

P60/DO YOU JOG/SWIM/CYCLE OR EXERCISE

YES NO TOTAL

STANDARD FED. ADMNSTRATV REGIONS

I NNEW ENGLND STS

II/NW YRK/NW JRY

III/MIO ATLANTIC

IV/SOUTH EAST

V/GREAT LAKES

VI/MIO SOUTH

VII/PLAINS

VIII/NW PLAINS

IX/WEST COAST

$X / N O R T H$ WEST

RESPONOENT'S AGE

$18-24$

$25-44$

45 AND OVER
59.26

57.14

61.90

56.90

61.58

56.25

52.83

46.88

61.98

58.82
40.74

42.86

38.10

43.10

38.42

43.75

47.17

53.13

38.02

41.18
54

112

105

174

203

112

53

32

121

34

RESPONDENT'S EDUCATION

$\begin{array}{llll}\text { LT HIGH SCHOOL } & 43.75 & 55.25 & 112 \\ \text { HIGH SCHOOL GRAD } & 56.06 & 43.94 & 371 \\ \text { SOME COLLEGE } & 64.81 & 35.19 & 270 \\ \text { COLLEGE GRAD } & 62.35 & 37.65 & 247\end{array}$

RESPONOENT'S SEX

$\begin{array}{lrcr}\text { MALE } & 60.52 & 39.48 & 499 \\ \text { FEMALE/HOME } & 56.38 & 43.62 & 243 \\ \text { FEMALE/EMPLOYED } & 56.98 & 43.02 & 258 \\ & & \\ \text { PRIMARY SOURCE OF FAMILY INCOME/C } & \\ & & & \\ \text { SLF-EMPL/WHITE C } & 61.86 & 38.14 & 430 \\ \text { BLUE-COLLAR } & 56.38 & 43.62 & 243 \\ \text { NOTEMPLOYED } & 54.46 & 45.54 & 213 \\ \text { OTHER } & 58.77 & 41.23 & 114 \\ \text { AGGREGATE } & 58.60 & 41.40 & 1000\end{array}$


TABLE 41 (continued)

TARRANCE \& ASSC. - SAFETY BELT (MOOD) - \#2017

$$
\begin{gathered}
\text { P60/DO YOU JOG/SWIM/CYCLE OR EXERCISE } \\
\text { YES NO TOTAL }
\end{gathered}
$$

\begin{tabular}{|c|c|c|c|}
\hline $\begin{array}{l}\text { NOT } \triangle P O L I C A B L E \\
\text { FREQ USERS/CNFRM } \\
\text { SMTM USER/LIKELY } \\
\text { SMTM USER/UNLKLY } \\
\text { INFREQ USR/LIKLY } \\
\text { INFREQ USR/CNFRM }\end{array}$ & $\begin{array}{l}67.85 \\
61.47 \\
64.13 \\
49.40 \\
47.68\end{array}$ & $\begin{array}{l}32.15 \\
39.53 \\
35.87 \\
50.60 \\
52.32\end{array}$ & $\begin{array}{r}311 \\
231 \\
92 \\
168 \\
151\end{array}$ \\
\hline \multicolumn{4}{|c|}{ P65/WHERE DO YOU LIVE } \\
\hline $\begin{array}{l}\text { IN A BIG CITY } \\
\text { SUBURBS/BIG CITY } \\
\text { IN A SMALL CITY } \\
\text { TOWN/VILLAGE } \\
\text { IN THE COUNTRY } \\
\text { OK/NO ANSWER. }\end{array}$ & $\begin{array}{r}60.85 \\
65.51 \\
55.36 \\
53.53 \\
53.54 \\
0.00\end{array}$ & $\begin{array}{r}39.15 \\
34.49 \\
44.64 \\
46.47 \\
46.40 \\
100.00\end{array}$ & $\begin{array}{r}189 \\
287 \\
224 \\
170 \\
127 \\
3\end{array}$ \\
\hline \multicolumn{4}{|c|}{ FREG-ONV-1 HR ORNKNG } \\
\hline $\begin{array}{l}\text { CAILY TC I/MONTH } \\
\text { SEVERAL TMS/YYR } \\
\text { NEVER }\end{array}$ & $\begin{array}{l}65.50 \\
64.77 \\
54.04\end{array}$ & $\begin{array}{l}33.50 \\
35.23 \\
45.96\end{array}$ & $\begin{array}{l}200 \\
193 \\
607\end{array}$ \\
\hline AGGREGATE & 58.60 & 41.40 & 1000 \\
\hline
\end{tabular}

USER CATAGURIES 
TABLE 42 - DEMOGRAPHICS BY HEALTH ACTIVITY TARRANCE \& ASSC. - SAFETY BELT (MOOO) - \#2017

P6I/UNDERTAKEN ANY HEALTH ACTVTIES/PST

YES NO TOTAL

STANDARO FED. AOMNSTRATV RECIIONS

$\begin{array}{lllr}\text { I/NEW ENGLND STS } & 68.52 & 31.48 & 54 \\ \text { II/NW YRK/NW JRY } & 57.14 & 42.86 & 112 \\ \text { III/MIO ATLANTIC } & 65.71 & 34.29 & 105 \\ \text { IV/SOUTH EAST } & 53.45 & 46.55 & 174 \\ \text { V/GREAT LAKES } & 53.69 & 46.31 & 203 \\ \text { VI/MID SOUTH } & 62.50 & 37.50 & 112 \\ \text { VII/PLAINS } & 62.26 & 37.74 & 53 \\ \text { VIII/NW PLAINS } & 75.00 & 25.00 & 32 \\ \text { IX/WEST COAST } & 57.85 & 42.15 & 121 \\ \text { X/NORTH WEST } & 52.94 & 47.06 & 34\end{array}$

RESPONDENTIS AGE

$\begin{array}{llll}18-24 & 70.15 & 29.85 & 134 \\ 25-44 & 61.89 & 38.11 & 475 \\ 45 \text { AND OVER } & 50.90 & 49.10 & 391\end{array}$

RESPONDENTIS EDUCATION

$\begin{array}{llll}\text { LT HIGH SCHOOL } & 41.96 & 58.04 & 112 \\ \text { HIGH SCHOOL GRAD } & 57.95 & 42.05 & 371 \\ \text { SOME COLLEGE } & 60.00 & 40.00 & 270 \\ \text { COLLEGE GRAO } & 65.99 & 34.01 & 247\end{array}$

RESPONDENT'S SEX

$\begin{array}{lrrr}\text { MALE } & 58.12 & 41.88 & 499 \\ \text { FEMALE/HOME } & 58.44 & 41.56 & 243 \\ \text { FEMALE/EMPLOYEO } & 60.08 & 39.92 & 258 \\ \text { PRIMARY SOURCE OF } & \text { FAMILY INCOME/C } & \\ \text { SLF-EMPL/WHITE C } & 63.26 & 36.74 & 430 \\ \text { BLUE-COLLAR } & 56.38 & 43.62 & 243 \\ \text { NOT EMPLOYED } & 53.05 & 46.95 & 213 \\ \text { OTHER } & 57.02 & 42.98 & 114 \\ \text { AGGREGATE } & 53.70 & 41.30 & 1000\end{array}$


TABLE 42 (continued)

TARRANCE \& ASSC. - SAFETY BELT (MOOD) - \#2017

P6I/UNDERTAKEN ANY HEALTH ACTVTIES/PST

YES NO TOTAL

USER CATAGURIES

NOT $\triangle P O L I C A B L E$

FREQ USERS/CNFRM

$\begin{array}{llr}53.67 & 36.33 & 311 \\ 62.77 & 37.23 & 231 \\ 57.61 & 42.39 & 92 \\ 55.36 & 44.64 & 168 \\ 48.34 & 51.66 & 151\end{array}$

SMTM USER/LIKELY

SMTM USER/UNLKLY

INFREQ USR/LIKLY

INFREQ USR/CNFRM

$48.34 \quad 51.66$

151

P65/WHERE DO YOU LIVE

$\begin{array}{lrrr}\text { IN A BIG CITY } & 56.08 & 43.92 & 189 \\ \text { SUBURBS/BIG CITY } & 54.46 & 35.54 & 287 \\ \text { IN A SMALL CITY } & 58.93 & 41.07 & 224 \\ \text { TONN/VILLAGE } & 55.88 & 44.12 & 170 \\ \text { IN THE COUNTRY } & 54.33 & 45.67 & 127 \\ \text { DK/NO ANSWER } & 0.00 & 100.00 & 3 \\ \text { FREO-ORV-1 HR DRNKNG } & & & \\ \text { DLILY TO I/MONTH } & 50.50 & 37.50 & 200 \\ \text { SEVERAL TMS/YR } & 58.03 & 41.97 & 193 \\ \text { REVER } & 58.32 & 41.68 & 607 \\ \text { AGGREGATE } & 58.70 & 41.30 & 1000\end{array}$


users. This data is supported by the focus group results. Both sets - strongly indicate that when the belt is psychologically positioned as a preventive health or wellness measure, its acceptability is much higher than when placed as a safety item.

One issue of utmost importance to the program that came out of the focus groups is the general annoyance with the child safety seat laws that are being passed. Most people felt that the laws were another governmental invasion. Again the laws were not seen as preventive health measures or even in the same light as child abuse laws. They were seen as the government interfering with how the parents raise their children. This perspective while not universal was common across all user groups and in many cases the most vocal parents were those who reported that they always used car safety seats.

No one in the groups argued that the laws wouldn't increase the use of seats, they felt strongly that it wasn't "right" for the government to interfere. When the car seat was placed in the same context as innoculations that children are given to prevent or reduce the negative effects of various childhood diseases most, if not all, of the opposition disappeared.

This strong negative reaction to the child safety seat laws, plus the findings of the same nature regarding the use of the safety belt, strongly suggests that the focus of all of the messages of any type that are developed to promote the use of occipant protection devices should be couched in the framework of wellness and preventive health.

In another area, an issue that continues to present an image problem is the fact that less than half of the respondents in the survey either disagreed or disagreed strongly with the following statement:

"I don't think seat belts work properly when they feel so loose around me when I fasten them." (see Table 43) 
TABLE 43 - DEMOGRAPHICS BY SEAT BELTS TOO LOOSE/DON'T WORK

TARRANCE \& ASSC. - SAFETY BELT (MOOD) - \#2017

PII/SEAT BELTS DONIT WORK IF TOO LOOSE

AGREE/ST AGREE UNSURE DISAGREE OISAGREE
RONGLY

TOTAL

STANDARC FED. ADMNSTRATV REGIONS

$\begin{array}{llllll}\text { I/NEW ENGLNO STS } & 12.96 & 18.52 & 12.96 & 29.63 & 25.93 \\ \text { IIINW YPK/NW JRY } & 18.75 & 21.43 & 12.50 & 21.43 & 25.89 \\ \text { III/MID ATLANTIC } & 19.05 & 19.05 & 10.48 & 18.10 & 33.33 \\ \text { IV/SOUTH EAST } & 21.84 & 20.11 & 20.69 & 20.11 & 17.24 \\ \text { V/GREAT LAKES } & 16.26 & 17.24 & 16.26 & 22.17 & 28.08 \\ \text { VI/MID SOUTH } & 18.75 & 19.64 & 16.96 & 17.86 & 26.79 \\ \text { VII/PLAINS } & 13.21 & 18.87 & 7.55 & 16.98 & 43.40 \\ \text { VIII/NW PLAINS } & 25.00 & 12.50 & 15.63 & 18.75 & 28.13 \\ \text { IX/WEST COAST } & 14.88 & 19.83 & 13.22 & 23.97 & 28.10 \\ \text { XINORTH WEST } & 14.71 & 11.76 & 8.82 & 29.41 & 35.29\end{array}$

54

112

105

174

203

112

53

32

121

34

RESPONDENT'S AGE

$\begin{array}{llllll}18-24 & 17.91 & 22.39 & 5.22 & 25.37 & 29.10 \\ 25-44 & 16.42 & 21.05 & 13.58 & 20.21 & 28.63 \\ 45 \text { AND OVER } & 19.44 & 14.83 & 19.44 & 21.23 & 25.06\end{array}$

134
475
391

RESPONDENT'S EDUCATION

$\begin{array}{lllllll}\text { LT HIGH SCHOOL } & 16.07 & 15.96 & 29.46 & 13.39 & 24.11 & 112 \\ \text { HIGH SCHOOL GRAD } & 21.56 & 19.33 & 12.67 & 22.54 & 24.80 & 371 \\ \text { SOME COLLEGE } & 15.19 & 20.74 & 13.33 & 20.00 & 30.74 & 270 \\ \text { COLLEGE GRAD } & 15.79 & 13.22 & 12.96 & 24.29 & 28.74 & 247\end{array}$

RESPONDENT'S SEX

$\begin{array}{lcccccc}\text { MALE } & 18.84 & 19.64 & 11.82 & 21.24 & 28.46 & 499 \\ \text { FEMALE/HOME } & 20.15 & 17.70 & 18.11 & 18.93 & 25.10 & 243 \\ \text { FEMALE/EMPLOYED } & 13.57 & 18.22 & 17.44 & 23.64 & 27.13 & 258 \\ \text { PRIMARY SOURCE OF } & \text { FAMILY INCOME/C } & & & & \\ & & & & & & \\ \text { SLF-EMPL/WHITE C } & 16.74 & 19.77 & 12.33 & 21.86 & 29.30 & 430 \\ \text { BLUE-COLLAR } & 18.93 & 20.58 & 13.99 & 20.58 & 25.93 & 243 \\ \text { NOTEMPLOYED } & 18.31 & 15.02 & 19.72 & 22.07 & 24.88 & 213 \\ \text { OTHER } & 18.42 & 18.42 & 16.67 & 19.30 & 27.19 & 114 \\ \text { AGGREGATE } & 17.80 & 18.80 & 14.80 & 21.30 & 27.30 & 1000\end{array}$


TABLE 43 (continued)

130

TARRANCE \& ASSC. - SAFETY BELT (MOOD) - \#2017

PII/SEAT BELTS DON'T WORK IF TOO LOOSE

AGREE/ST AGREE UNSURE DISAGREE DISAGREE
RONGLY

TOTA'

USER CATAGORIES

NOT APPLICABLE

FREQ USERS/CNFRM

SMTM USER/LIKELY

$17.36 \quad 17.94$

0.11

9.52

7.78

SMTM USER/UNLKLY

16.88

19.05

21.43

INFREQ USP/CNFRM

$18.45 \quad 14.29$

15.89

21.85

30.46

$$
\begin{array}{ll}
25.72 & 30.87 \\
19.91 & 34.63 \\
16.30 & 29.35 \\
23.21 & 22.62 \\
13.91 & 17.88
\end{array}
$$

31.

23

9

16

PS5/WHERE DO YOU LIVE

$\begin{array}{llllll}\text { IN A BIG CITY } & 15.87 & 18.52 & 16.40 & 21.69 & 27.51 \\ \text { SUBURBSIBIG CITY } & 16.03 & 20.21 & 10.80 & 20.91 & 32.06 \\ \text { IN A SIALLCITY } & 20.09 & 17.86 & 15.63 & 20.54 & 25.89 \\ \text { TOWNIVILLAGE } & 18.24 & 21.76 & 17.65 & 20.59 & 21.76 \\ \text { IN THE COUNTRY } & 20.47 & 13.39 & 15.75 & 23.62 & 26.77 \\ \text { DKINO ANSIWER } & 0.00 & 33.33 & 33.33 & 33.33 & 0.00\end{array}$

FREQ-DRV-I HR DRNKNG

OAILY TO I/MONTH

SEVERAL TMS/YR

NEVER

$\begin{array}{lllll}17.00 & 18.50 & 14.50 & 24.50 & 29.50 \\ 19.69 & 18.65 & 9.33 & 24.87 & 27.46 \\ 17.46 & 18.95 & 17.96 & 19.11 & 26.52 \\ 17.80 & 18.80 & 14.90 & 21.30 & 27.30\end{array}$

18

28

22

17

12.

AGGREGATE

17.80

18.80

14.90

21.30

$27 \cdot 30$

20

19

$60 \%$

1000 
If people don't believe that their belt operates properly then what is the inducement to start wearing the belt? This lack of understanding of how the inertial belt works is real and becomes evident when the topic is addressed in focus groups. The number of people who have no idea why they can pull on their belt and have it unreel unless it's broken is amazine. Once they are told how it works and that it was designed to increase comfort and convenience, they are more inclined to listen to why they should use the belt. They now know that theirs works!

The data on a number of attitudinal items are presented in Tables 44 and 48. These more general attitudinal items show the same spread amongst the user categories as do the other items which have been previously dis. cussed. It should be noted however, that they do not differentiate between the three drinking groups. 
132

TABLE 44 - DEMOGRAPHICS BY NO ACCIDENTS/DRIVE DEFENSIVELY

TARRANCE \& ASSC. - SAFETY BELT (MOOD) - \#2017

PT/WON T HAVE ACCIDENT/I DRIVE DEFENSIV

AGREE/ST AGREE UNSURE DISAGREE DISAGREE RONGLY ISTRNGLY

TOTA'

STANDARD FED. ADMNSTRATV REGIONS

I/NEW ENGLND STS

II/NW YRK/NW JRY

III/MID ATLANTIC

IV/SOUTH EAST

V/GREAT LAKES

VI/MID SOUTH

VII/PLAINS

VIII/NW PLAINS

IX/WEST COAST

$X / N O R T H$ WEST
$24.07 \quad 11.1$

$16.07 \quad 10.71$

14.29

13.79

17.73

14.29

13.21

15.63

12.40

8.82
8.57

8.62

8.87

5.2.

7.55

12.50

11.57

2.94
1.85

5.36

6.67

2.30

6.90

3.57

7.55

0.00

3.31

5.88
$18: 52$

28.57

20.95

25.86

21.18

31.25

26.42

25.00

34.71

26.47
44.44

39.29

49.52

49.43

45.32

44.64

45.28

46.88

38.02

55.88
5
11
10
17
20
11
5
3
12
3

13
47

39

48.00

41.69
1.49
4.42

5.88
27.37

24.04
7.14

4.85

4.81

2.83
17.96

25.61

25.56

30.77
37.50 45.01 48.89 44.94
11

37

27

24

COLLEGE GRAD

$11.34 \quad 10.12$

RESPONDENT'S SEX

$\begin{array}{llr}\text { MALE } & 16.63 & 11.4 \\ \text { FEMALE/HOME } & 15.23 & 9.05 \\ \text { FEMALE/EMPLOYED } & 12.40 & 4.26 \\ \text { PRIMARY SOURCE OF FAMILY INCOME/C }\end{array}$

SLF-EMPL/WHITE C
BLUE-COLLAR
NOT EMPLOYED
OTHER

AGGREGATE

14.88
16.46
14.55
14.91

8.14

7.41

11.27

11.40

3.81

$7: 00$

24.85

26.34

43.29

42.39

3.88

27.91

51.55

49
24
25

43
24
21
11

100

46.05

47.74

41.78

42.98

9.00

4.60

26.00

45.20 
TABLE 44 (continued)

TARRANCE \& ASSC. - SAFETY BELT (MOOO) - \#2017.

P7/WON T HAVE ACCIDENT/I DRIVE DEFENSIV

AGREE/ST AGREE UNSURE DISAGREE DISAGREE
RONGLY ISTRNGLY

TOTAL

USEP CATAGORIES

NOT $\triangle P P L I C A B L E$

FREQ USERS/CNFFM

SMTM USER/LIKELY

SMTM USER/UNLKLY

INFREQ USRALIKLY

$\begin{array}{rr}15.11 & 8.04 \\ 13.42 & 6.93 \\ 18.48 & 9.78 \\ 14.29 & 7.74 \\ 17.88 & 15.23\end{array}$

3.22
4.33
3.26
4.76
5.96

$31.5 i$
19.48
18.48
28.57
24.50

42.12

55.84

50.00

44.64

INFREQ USR/CNFRM

17.88

36.42

311

231

92

168

151

DS5/WHERE DO YOU LIVE

IN 2 BIG CITY
SUBURBS/BIG CITY
IN A SMALL CITY
TONN IVILLAGE
IN THE COUNTRY
DXINO ANSWER

$\begin{array}{rr}16.40 & 7.41 \\ 14.29 & 10.80 \\ 15.63 & 6.25 \\ 13.53 & 11.76 \\ 17.32 & 7.87 \\ 0.00 & 33.33\end{array}$

7.41

27.51

3.83

4.46

4.12

28.57

41.27

23.66

42.51

25.88

50.00

3.15

21.26

0.00

44.71

50.39

66.67

0.00

189

287

224

170

127

3

FREQ-DRV-I HR DRNKNG

DAILY TO I/MONTH
SEVERAL TMS/YR
NEVER

14.50

16.06

5.50

3.81

3.50
3.63

25.00

27.46

50.50

44.04

25.86

43.82

200

5.27

9.00

4.60

26.00

45.20

193

607

AGGREGATE

15.20

4.60

1000 
134

TABLE 45 - DEMOGRAPHICS BY BELT USE VS. INSURANCE

TARRANCE \& ASSC. - SAFETY BELT (MOOO) - $\# 2017$

PIZ/FASTEN SEAT BELTS VS. AUTO INSURANC

MR JONES MR JONES UNSURE MR SMITH MR SMITH
/STRTRLY

TOTA'

STANDAKD FED. SOMNSTRATV REGIONS

$\begin{array}{lrrrrr}\text { I/NEW ENGLNO STS } & 40.74 & 24.07 & 3.70 & 12.96 & 18.52 \\ \text { II/NW YRK/NW JRY } & 52.68 & 24.11 & 3.57 & 5.36 & 14.29 \\ \text { III/MID ATLANTIC } & 53.33 & 19.05 & 3.81 & 7.62 & 16.19 \\ \text { IV/SOUTH EAST } & 44.83 & 16.09 & 10.92 & 13.22 & 14.94 \\ \text { V/GREAT LAKES } & 48.77 & 15.27 & 5.91 & 11.82 & 18.23 \\ \text { VI/MID SOUTH } & 50.89 & 18.75 & 4.46 & 10.71 & 15.18 \\ \text { VII/PLAINS } & 52.83 & 16.98 & 3.77 & 11.32 & 15.09 \\ \text { VIIINN PLAINS } & 53.13 & 21.88 & 3.13 & 9.38 & 12.50 \\ \text { IX/NEST COAST } & 52.07 & 18.18 & 7.44 & 10.74 & 11.57 \\ \text { X/NORTH WEST } & 55.88 & 14.71 & 2.94 & 17.65 & 8.82\end{array}$

RESPONCENTIS AGE

$\begin{array}{lllllll}18-24 & 47.01 & 22.39 & 2.99 & 14.93 & 12.69 & 13 \\ 25-44 & 52.00 & 18.95 & 4.00 & 11.16 & 13.89 & 47 \\ 45 \text { AND OVER } & 48.08 & 16.11 & 9.21 & 8.95 & 17.65 & 39 .\end{array}$

RESPONDENTIS EDUCATION

\begin{tabular}{|c|c|c|c|c|c|c|}
\hline $\begin{array}{l}\text { LT HIGH SCHOCL } \\
\text { HIGH SCHOOL GRAO } \\
\text { SOME COLLEGE } \\
\text { COLLEGE GRAD }\end{array}$ & $\begin{array}{l}41.96 \\
48.25 \\
50.74 \\
54.66\end{array}$ & $\begin{array}{l}10.71 \\
17.79 \\
21.85 \\
18.62\end{array}$ & $\begin{array}{r}12.50 \\
6.47 \\
5.93 \\
2.02\end{array}$ & $\begin{array}{r}11.61 \\
11.32 \\
8.89 \\
11.74\end{array}$ & $\begin{array}{l}23.21 \\
16.17 \\
12.59 \\
12.96\end{array}$ & $\begin{array}{l}11 \\
37 \\
27 \\
24\end{array}$ \\
\hline \multicolumn{7}{|l|}{ RESPONDENT'S SEX } \\
\hline $\begin{array}{l}\text { MOLE } \\
\text { FEMALE /HOME } \\
\text { FEMALE/EMPLOYEO }\end{array}$ & $\begin{array}{l}45.69 \\
54.32 \\
53.49\end{array}$ & $\begin{array}{l}19.44 \\
14.40 \\
19.77\end{array}$ & $\begin{array}{l}6.21 \\
7.00 \\
4.26\end{array}$ & $\begin{array}{r}11.82 \\
10.29 \\
9.30\end{array}$ & $\begin{array}{l}16.83 \\
13.99 \\
13.18\end{array}$ & $\begin{array}{l}49 \\
24 \\
25\end{array}$ \\
\hline \multicolumn{7}{|c|}{ PRIMARY SOURCE OF FAMILY INCOME/C } \\
\hline $\begin{array}{l}\text { SLF-EMPL/WHITE C } \\
\text { BLUE-COLLAR } \\
\text { NOT EMPLOYED } \\
\text { OTHER }\end{array}$ & $\begin{array}{l}50.47 \\
48.56 \\
48.36 \\
52.63\end{array}$ & $\begin{array}{l}22.09 \\
15.23 \\
15.02 \\
16.67\end{array}$ & $\begin{array}{l}5.58 \\
4.53 \\
9.39 \\
3.51\end{array}$ & $\begin{array}{l}9.07 \\
12.76 \\
10.80 \\
13.16\end{array}$ & $\begin{array}{l}12.79 \\
18.93 \\
16.43 \\
14.04\end{array}$ & $\begin{array}{l}43 \\
24 \\
2 ! \\
11\end{array}$ \\
\hline$\triangle G G R E G \Delta T E$ & 49.80 & 18.30 & 5.90 & 10.80 & 15.20 & 100 \\
\hline
\end{tabular}


TABLE 45 (continued)

TARRANCE \& ASSC. - SAFETY BELT (MOOD) - \#2017

PIZ/FASTEN SEAT BELTS VS. AUTO INSURANC

MR JONES MR JONES UNSURE MR SMITH MR SMITH ISTRNGLY

ISTRNGLY

TOTAL

USER CATAGORIES

NOT $\triangle P P L I C A B L E$

FPEO USEPS/CNFRM

$63.3 \dot{4} \quad 18.0 \dot{1}$

$56.28 \quad 18.61$

$57.61 \quad 15.22$

$39.29 \quad 19.05$

$25.17 \quad 18.54$

$4.1 \dot{8}$

4.33

5.43

5.36

9.27

INFREO USR/CNFRM

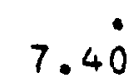

8.66

8.70

13.10

17.22

3.70

5.92

10.58

i 0.45

12.50

12.35

7.09

0.00

18.11

33.33
7.06

7.09

60.67
12.17

13.59

17.86

14.12

20.47

0.00
189

287

224

170

127

3

FREQ-DRV-1 HR DRNKNG

DAILY TO I/MONTH

SEVEFAL TMS/YR

NEVER

AUGREGATE
$48.50 \quad 17.00$

$51.81 \quad 18.13$

$49.59 \quad 13.78$

$49.80 \quad 18.30$
1.00

6.22

7.41

5.90
15.50

10.36

9.39

10.80
18.00

13.47

14.83

15.20
200

193 607

1000 
TABLE 46 - DEMOGRAPHICS BY BELT USE: SAFETY VS. IMPOSITION

TARRANCE \& ASSC. - SAFETY BELT (MOOD) - \#2017

P13/SEAT BELTS/SAFETY VS. SEAT BELTS/IM

MR JONES MR JONES UNSURE MR SMITH MR SMITH ISTRNGLY

ISTRNGLY

TOTA'

STANDARD FED. ADMNSTRATV REGIONS

I/NEW ENGLND STS

II/NWW YRK/NW JRYY

III/MID ATLANTIC

IV/SOUTH EAST

V/GREAT LAKES

VI/MID SOUTH

VII/PLAINS

VIII/NW PLAINS

IX/WEST COAST

$X / N O P T H$ WEST

RESPONDENT'S AGE

$18-24$
$25-44$
45 AND OVER
RESPONDENT'S EDUCATION

$\begin{array}{ll}50.00 & 12.96 \\ 56.25 & 23.21 \\ 54.29 & 20.95 \\ 51.72 & 21.26 \\ 55.67 & 14.29 \\ 51.79 & 22.32 \\ 41.51 & 28.30 \\ 56.25 & 18.75 \\ 55.37 & 21.49 \\ 58.82 & 17.65\end{array}$

53.73

50.32

57.29
23.13

20.00

18.67
LT KIGH SCHOOL

HIGH SCHOOL GRAD

SOME COLLEGE

COLLEGE GRAD
$52.68 \quad 15.18$

$55.26 \quad 17.52$

$49.63 \quad 24.07$

55.47
3.70

0.89

2.86

5.17

3.45

1.79

7.55

0.00

1.65

5.88

$$
\begin{array}{r}
14.81 \\
12.50 \\
5.71 \\
8.05 \\
9.85 \\
11.61 \\
13.21 \\
15.63 \\
13.22 \\
11.76
\end{array}
$$

18.52

7.14

16.19

13.79

16.75

12.50

9.43

9.38

8.26

5.88
1.49

3.37

3.58
12.69

11.37

9.21
8.96

14.95

11.25
5
11
10
17
20
11
5
3
12
2

13

47

39

11
37
27
24

$\begin{array}{lll}7.14 & 4.46 & 20.54\end{array}$

$2.70 \quad 12.13 \quad 12.40$

$\begin{array}{lll}3.33 & 10.37 & 12.59\end{array}$

2.02

11.74

9.72

PESPONDENT'S SEX

$\begin{array}{lrrrrrr}\text { MALE } & 47.70 & 20.84 & 4.21 & 13.23 & 14.03 & 49 \\ \text { FEMALE/HOME } & 63.37 & 15.23 & 1.65 & 7.82 & 11.93 & 24 \\ \text { FEMALE/EMPLOYED } & 55.43 & 22.48 & 2.71 & 8.53 & 10.85 & 25 \\ \text { PRIMARY SOURCE OF FAMILY INCOME/C } & & & & \\ & & & & & & \\ \text { SLF-EMPL/WHITE C } & 51.63 & 22.33 & 1.86 & 10.93 & 13.26 & 43 \\ \text { BLUE-COLLAP } & 52.26 & 18.11 & 4.12 & 11.52 & 13.99 & 24 \\ \text { NOTEMPLOYED } & 56.81 & 21.13 & 3.76 & 7.04 & 11.27 & 21 \\ \text { OTHER } & 57.02 & 12.28 & 5.26 & 14.91 & 10.53 & 11 \\ \text { AGGREGATE } & 53.50 & 19.90 & 3.20 & 10.70 & 12.70 & 100\end{array}$


TABLE 46 (continued)

TARRANCE \& ASSC. - SAFETY BELT (MOOD) - \#2017

PI3/SEAT BELTS/SAFETY VS. SEAT BELTS/IM

ME JONES MR JONES UNSURE MR SMITH MR SMITH

ISTRNGLY

ISTRNGLY

TOTAL

USER CATAGORIES

NOT APPLICABLE

FREQ USERS/CNFRM

$70.4 \dot{2} \quad 19.9 \dot{7}$

$0.6 \dot{4}$

$60.17 \quad 22.51$

1.30

15.30

5.43

5.95

45.83

17.86

22.52

17.88

5.96

4.82

8.66

6.52

17.25

20.53

INFRED USR/CNFRM

PS5/WHERE DO YOU

LIVE

IV A ZIG CITY

SUBUPBS/EIG CITY

IN A SUALL CITY

TOWN/VILLAGE

IY THE COUNTRY

DK/NO ANSWER

$\begin{array}{ll}56.61 & 17.46 \\ 51.22 & 20.21 \\ 56.70 & 20.54 \\ 51.76 & 21.75 \\ 51.18 & 18.90 \\ 33.33 & 33.33\end{array}$

FREQ-URV-1 HR DRNKNG
48.00

48.70

56.84

17.50

25.91

18.78

$53.50 \quad 19.90$
4.23
2.79
1.34
3.53
4.72
33.33

$$
\begin{aligned}
& 11.11 \\
& 13.24 \\
& 10.27 \\
& 7.65 \\
& 9.45 \\
& 0.00
\end{aligned}
$$

10.58
12.54
11.16
15.29
15.75
0.00

189

287

224

170

127

3

AGGREGATE

53.50

1.50
1.04
4.45

16.50
11.92
8.40

16.50

12.44

11.53

200

193

607

$3.20 \quad 10.70 \quad 12.70$

1000 
TABLE 47 - DEMOGRAPHICS BY BUZZER: REMINDER VS. IGNORE TARRANCE \& ASSC. - SAFETY BELT (MOOD) - \#2017.

$$
\begin{aligned}
& \text { PI4/BUZZER IS A REMINOER VS. BUZZER IGN } \\
& \text { MR JONES MR JONES UNSURE MR SMITH MR SMITH } \\
& \text { ISTRNGLY }
\end{aligned}
$$

TOTAI

STANDAPO FEO. ADMNSTRATV REGIONS

$\begin{array}{llllll}\text { I/NEW ENGLND STS } & 44.44 & 15.67 & 7.41 & 12.96 & 18.52 \\ \text { II/NW YRK/NW JRY } & 48.21 & 20.54 & 2.68 & 8.93 & 19.64 \\ \text { III/MID ATLANTIC } & 49.52 & 17.14 & 2.36 & 13.33 & 17.14 \\ \text { IV/SOUTH EAST } & 48.85 & 15.52 & 6.90 & 1.4 .37 & 14.37 \\ \text { VIOREAT LAKES } & 49.26 & 12.32 & 5.91 & 16.75 & 15.76 \\ \text { VI/MID SOUTH } & 50.00 & 14.29 & 3.57 & 16.96 & 15.18 \\ \text { VII/DLAINS } & 43.40 & 15.09 & 9.43 & 11.32 & 20.75 \\ \text { VIII/NW PLAINS } & 59.38 & 12.50 & 0.00 & 9.38 & 18.75 \\ \text { IX/NEST COAST } & 52.89 & 22.31 & 4.13 & 9.92 & 10.74 \\ \text { X/NORTH WEST } & 50.00 & 20.59 & 2.94 & 17.55 & 8.82\end{array}$

5
11
10
17
20
11
5
3
12
3

RESPONOENT'S AGE

$\begin{array}{llllll}18-24 & 45.52 & 20.15 & 1.49 & 16.42 & 16.42 \\ 25-44 & 45.68 & 15.16 & 4.84 & 15.79 & 18.53 \\ 45 \text { AND OVER } & 55.24 & 16.62 & 6.14 & 9.97 & 12.02\end{array}$

PESPONDENT'S EDUCATION

$\begin{array}{llllll}\text { LT HIGH SCHOOL } & 50.89 & 11.61 & 10.71 & 6.25 & 20.54 \\ \text { HIGH SCHOOL GRAD } & 50.40 & 15.09 & 3.77 & 14.29 & 16.44 \\ \text { SOME COLLEGE } & 45.56 & 19.26 & 3.70 & 14.81 & 16.67 \\ \text { COLLEGE GRAD } & 51.42 & 17.41 & 5.26 & 14.57 & 11.34\end{array}$

RESPONDENT'S SEX

$\begin{array}{llllll}\text { MALE } & 43.49 & 17.03 & 4.61 & 15.63 & 19.24 \\ \text { FEMALE/HOME } & 60.49 & 12.35 & 5.76 & 9.88 & 11.52 \\ \text { FEMALE/EMPLOYED } & 50.39 & 18.99 & 4.65 & 13.18 & 12.79\end{array}$

\begin{tabular}{|c|c|c|c|c|}
\hline $\begin{array}{l}\text { SLF-EMPL/WHITE C } \\
\text { BLUE-COLLAR } \\
\text { NOT EMPLOYED } \\
\text { OTHER }\end{array}$ & $\begin{array}{l}48 \cdot 60 \\
47 \cdot 74 \\
51 \cdot 64 \\
51 \cdot 75\end{array}$ & $\begin{array}{l}19.77 \\
14.81 \\
14.55 \\
10.53\end{array}$ & $\begin{array}{l}4.42 \\
3.70 \\
7.04 \\
5.26\end{array}$ & $\begin{array}{l}13.26 \\
13.99 \\
12.21 \\
16.67\end{array}$ \\
\hline AGGREGATE & 49.40 & 16.40 & 4.90 & 13.60 \\
\hline
\end{tabular}

PRIMARY SOURCE OF FAMILY INCOME/C

430 243 213
114 
TABLE 47 (continued)

TARRANCE \& ASSC. - SAFETY BELT (MOOD) - \#2017

\section{P14/BUZZER IS A REMINDER VS. BUZZER IGN}

MR JONES MR JONES UNSURE MR SMITH MR SMITH ISTRNGLY

USER CATAGORIES

NOT $\triangle P P L I C A B L E$

FREQ USERS/CAFRM

SMTM USEF/LIKELY

SMTM USER/UNLKLY

INFPEO USR/LIKLY

INFREO USR/CNFPM

$\begin{array}{ll}77.49 & 15.4 \dot{3} \\ 48.05 & 23.38 \\ 51.09 & 15.22 \\ 32.14 & 11.90 \\ 18.54 & 11.26\end{array}$

PS5/WHERE DO YOU LIVE

IN A BIG CITY
SUEURSS/OIG CITY
IN A SIALL CITY
TOWN/VILLAGE
IN THE COUNTFY
OK/NO ANSWER
FREO-ONV-I HP DRNKNG
OAILY TO I/MONTH
SEVEPAL TMS/YQ
NEVER

AGGREGATE

$\begin{array}{ll}56.61 & 11.11 \\ 46.69 & 20.21 \\ 49.55 & 16.07 \\ 47.06 & 13.82 \\ 48.03 & 13.39 \\ 33.33 & 0.00\end{array}$

5.29

4.53

4.46

5.29

5.51

0.00

14.81

12.20

14.73

10.59

16.54

33.33

12.17

16.38

15.18

18.24

16.54

33.33

39.00

47.15

53.54

14.50

22.28

15.16

$49.40 \quad 16.40$

2.00

20.50

11.40

24.00

12.03

15.03

13.18

13.60

15.70

0.10

4.90
311

231

$9 ?$

168

151

189

287

224

170

127

3

4.15

200

193

607

1000 
TABLE 48 - DEMOGRAPHICS BY USE BELT: LESS/MORE SERIOUS INJURIES

\section{TARRANCE \& ASSC. - SAFETY BELT (MOOD) - \$2017}

PIS/BELTS/LESS INJURIES VS. SEVERE INJU

MR JONES MR JONES UNSURE MR SMITH MR SMITH ISTRNGLY

STANDARD FED. ADMNSTRATV REGIONS

\section{I /NEW ENGLNO STS \\ II $/ N W$ YRK/NW JRY \\ III/MIO ATLANTIC \\ IV/SOUTH, EAST \\ VIGREAT LAKES \\ $V I / M I D$ SOUTH \\ VII/PLAINS \\ VIII/NW PLAINS \\ IX/NEST COAST \\ $X / N O R T H$ WEST}

$\begin{array}{ll}59.26 & 18.52 \\ 64.29 & 19.64 \\ 60.00 & 20.95 \\ 63.22 & 12.07 \\ 65.02 & 11.33 \\ 58.93 & 19.64 \\ 64.15 & 20.75 \\ 81.25 & 12.50 \\ 61.98 & 23.97 \\ 73.53 & 14.71\end{array}$

RESPONDENT'S AGE

$\begin{array}{ll}61.94 & 23.13 \\ 66.95 & 14.53 \\ 59.85 & 17.65\end{array}$

45 ANO OVER

12.96
9.82
4.76
10.92
11.82
9.82
7.55
3.13
8.26
2.94

3.70

1.79

4.76

6.32

3.45

6.25

0.00

0.00

1.65

5.88

4.48

8.21

12.28

5.22
3.37
$3 \cdot 84$

5.22

6.95

6.39

5.56

4.46

9.52

7.47

8.37

5.36

7.55

3.13

4.13

2.94

3.84

3.57

4.58

4.07

2.43

9.82

7.55

7.04

2.83
5.93

4.86
18.33

17.41

17.00
17.04

13.58

15.89
4.01

3.70

3.49
6.41

8.23

5.04
499

243

258

PRIMARY, SOURCE OF FAMILY INCOME/C

SLF - EMPL/WHI
BLUE-COLLAR
NOT EMPLOYEO
OTHER

AGGREGATE
65.35

62.96

59.15

65.79

63.50
17.91

15.23

17.37

i5. 79

16.90
6.98

11.52

11.74

8.77

9.30
4.19

3.70

2.35

5.26

3.80
5.58

6.58

9.39

4.39

6.50
112

371

270

247
3.

3 
TABLE 48 (continued)

TARRANCE \& ASSC. - SAFETY BELT (MOOD) - $\$ 2017$

P15/3ELTS/LESS INJURIES VS. SEVERE INJU

MR JDHES MR JONES UNSURE MR SMITH MR SMITH ISTRNGLY

ISTRNGLY

TOTAL

USER CATAGORIES

NOT APPLICABLE

FREO USERS/CNFRM

SMTM USER/LIKELY

SMTM USER/UNLKLY

INFREQ USP LIKLY

INFREQ USR/CNFRM

$\begin{array}{ll}80.71 & 14.79 \\ 74.03 & 15.88 \\ 63.04 & 20.65 \\ 50.00 & 19.45 \\ 33.11 & 15.56\end{array}$

$2.5 \dot{7}$

6.06

4.78

12.50

19.87

15.56
$1.2 \dot{9}$

0.43

2.17

8.33

9.27

3.17

3.14

4.46

4.12

3.74

33.33
5.29

5.92

7.14

7.06

7.87

0.00
$31 i$

231

92

168

151
IV THE COUNTFY

DK/NO MISSWER
59.84

33.33
12.60

0.00
15.41

33.33
189

287

224

170

127

3

FREQ-DHV-1 HR ORNKNG

DAILY TO 1/MONTH

SEVERAL TMS/YR

NEVER

AGGREGATE
$56.50 \quad 19.50$

$69.95 \quad 15.58$

60.46

16.47

$63.50 \quad 15.90$
6.00

6.22

11.37

9.30
3.00

3.11

4.28

3.80
6.00

4.15

7.41

5.50
200

193

607

1000 


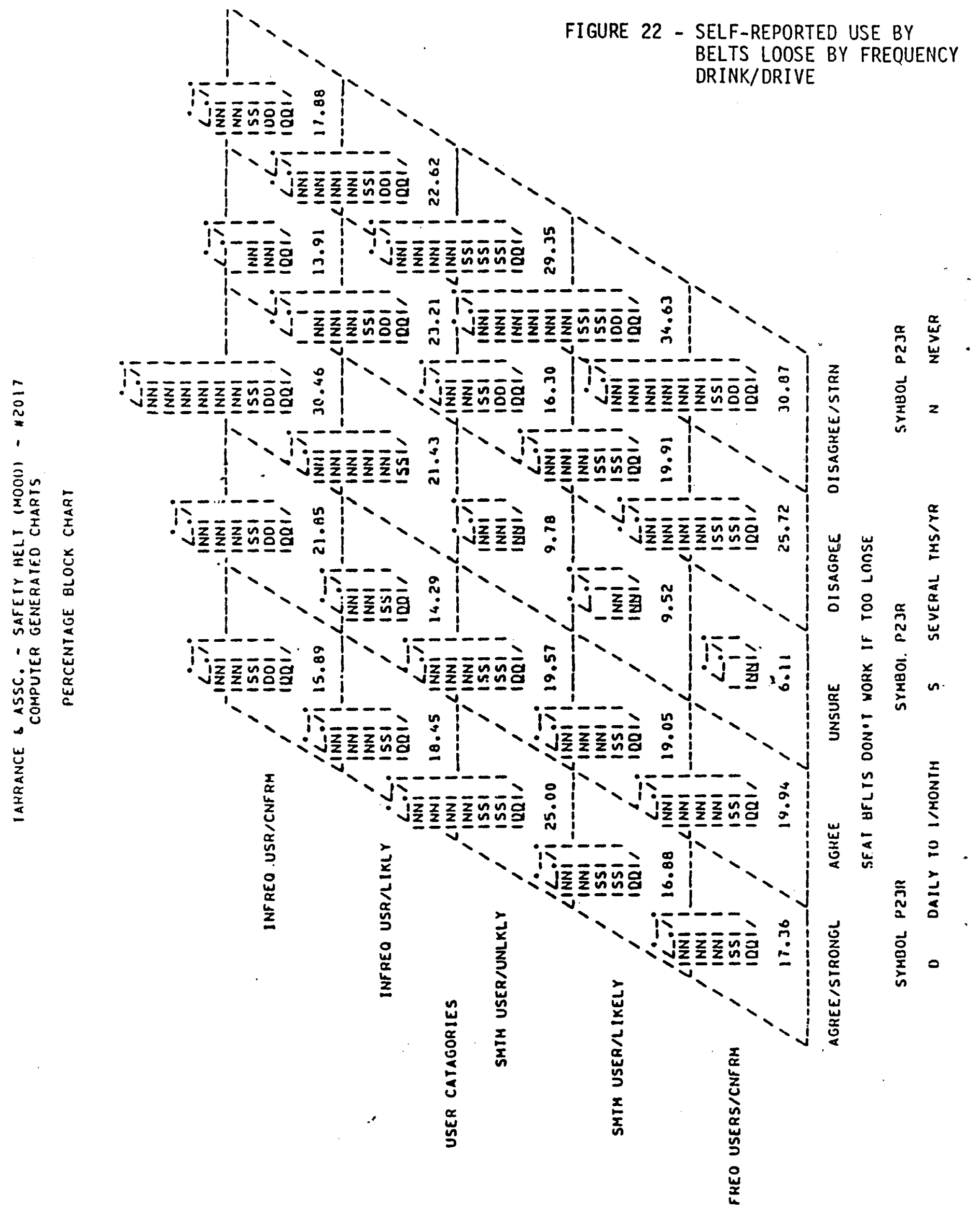



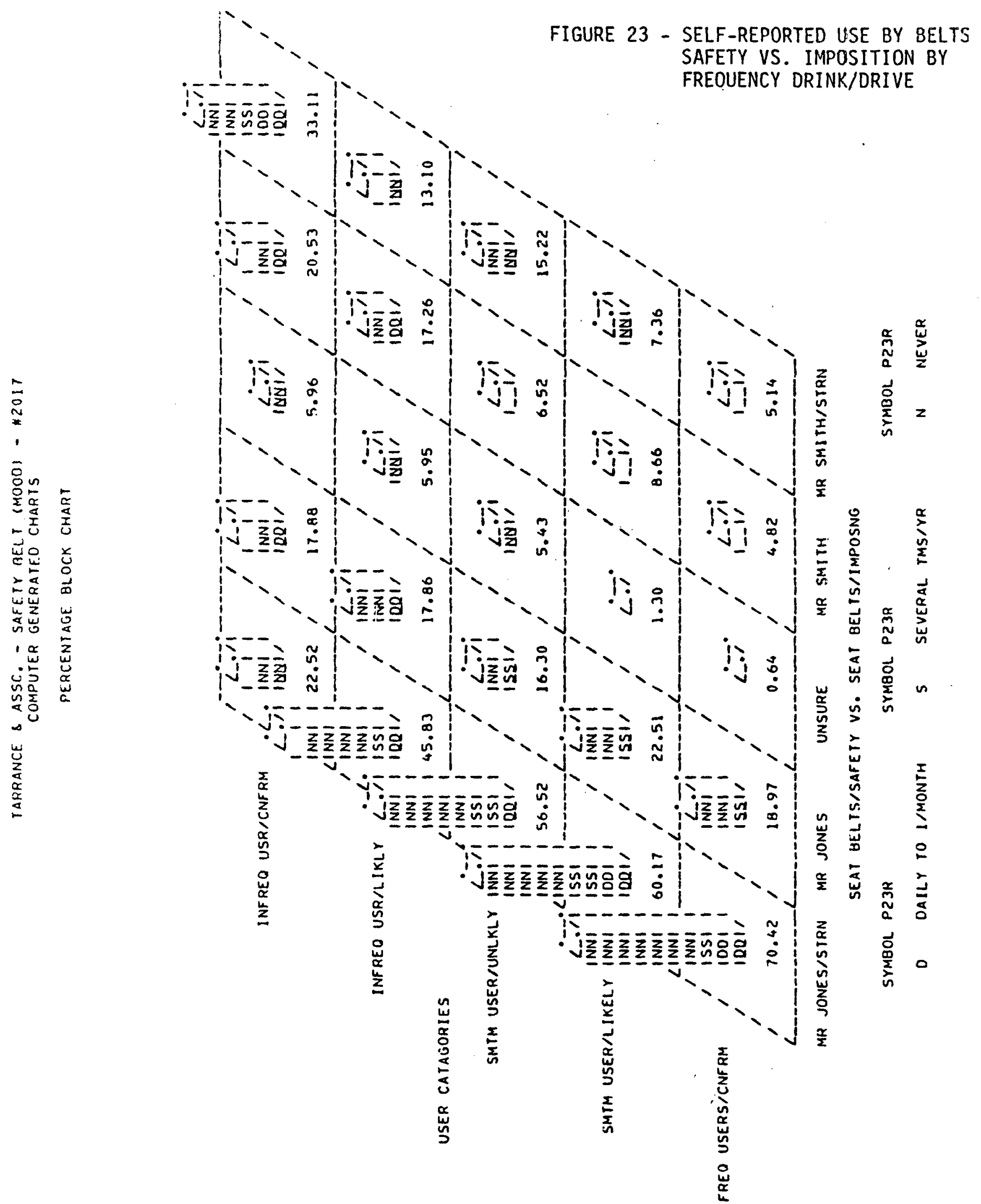

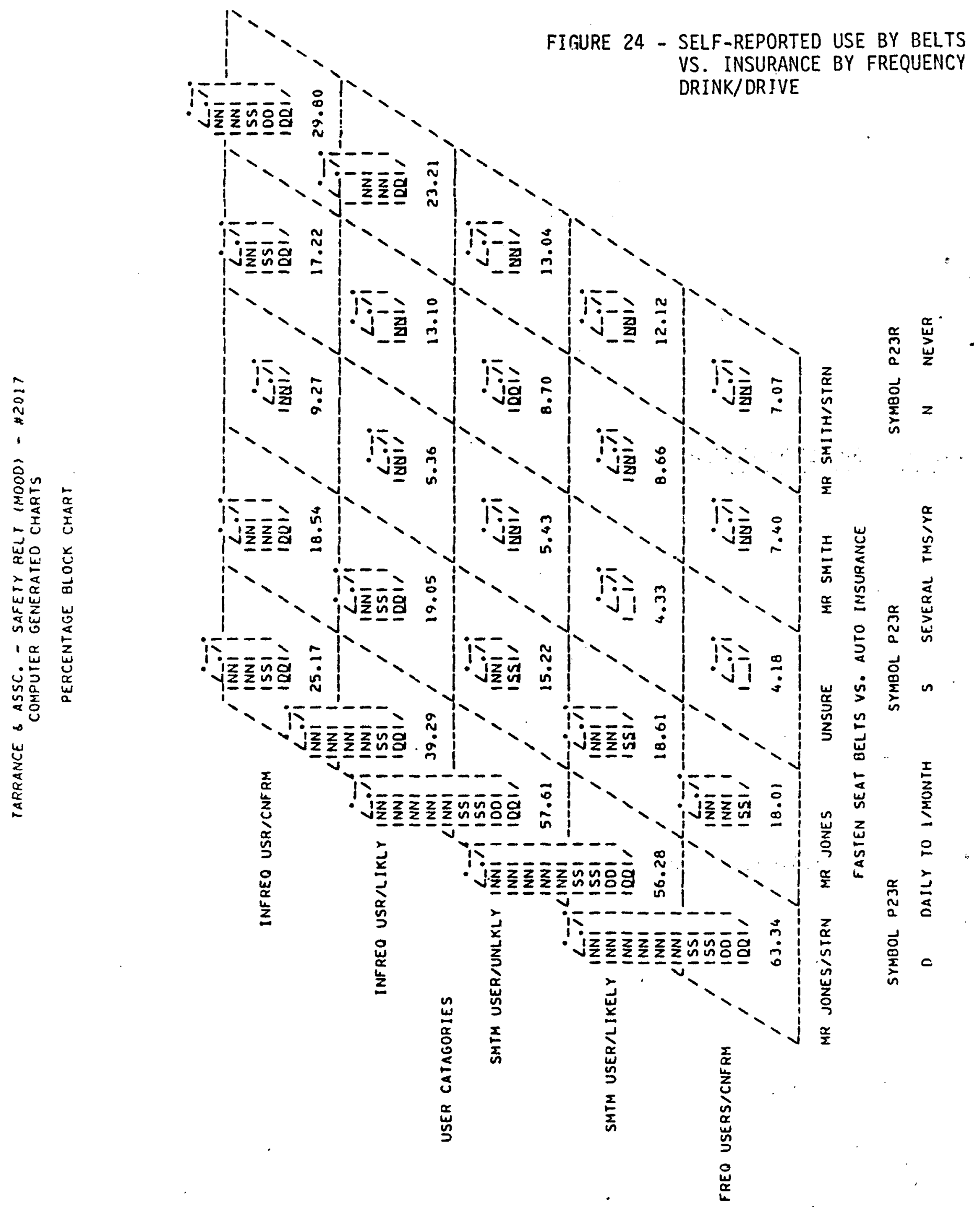

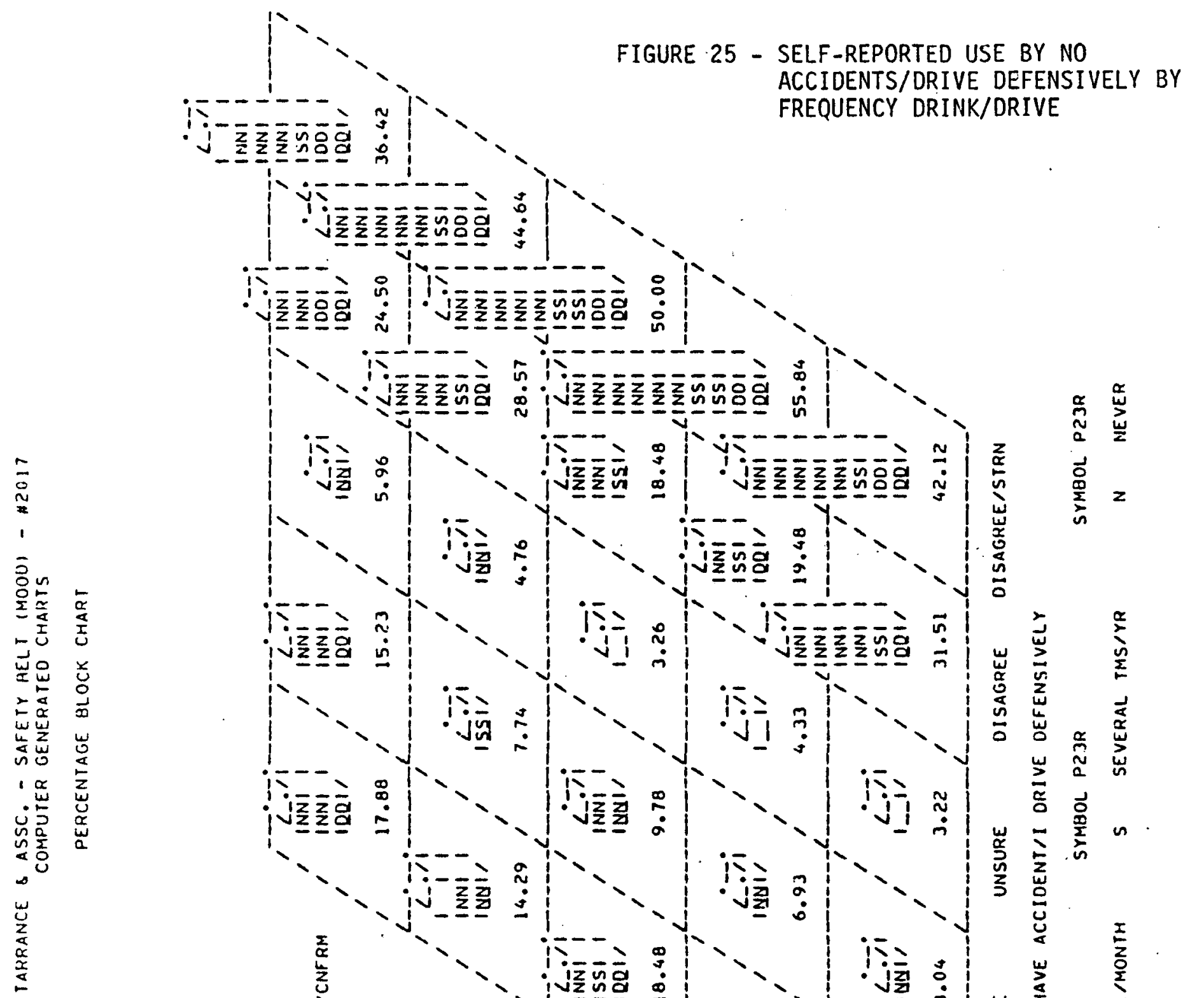
MOTHERS AGAINST ORUNK ORIVING

Based on the results of a series of focus groups that were conducted prior to the survey, a number of questions were added regarding Mothers Against Drunk Driving (or MADD). The reason that this group was singled out was based on the results of the focus group efforts. When asked what group or organization was doing the most to overcome or reduce the drunk driving problem, the univeral answer that was given by the focus group participants was MADD. When asked if they could name any other group, none of the respondents could. When RID and PARKIT were mentioned, no one recognized these acronyms. When asked about what impact federal, state or local governments were having, the respondents all talked about how MADO was pushing the state governments to action.

Clearly, MADD was seen in the focus groups as the cultural change agent which has been responsible for the changes in attitudes about drinking and driving. Based on these results, the following questions relating to MADD were included in the survey (see Tables 49 to 52 ).

- Have you ever heard of Mothers Against Drunk Driving or. MADD?

- If yes, can you tell me what MADD is trying to do?

- Do you think Mothers Against Drunk Driving is successful?

- If yes, and in your own words, what are one or two reasons why you feel that way?

Eight-four percent of all respondents had heard of MADD. In terms of demographic characteristics those with less than a high school education were less aware of MADD than the general public as were those who were unemployed. Females were somewhat more aware than men but $80 \%$ of the men had heard of MADD. Safety belt usage didn't tend to be important in terms of being aware of MADD, but then why should it? In terms of frequency of drinking and driving, those who drank were more aware of MADD than those who didn't. Clearly, MADD is getting to one of the target groups at which it is aiming. 
FIGURE 26 - FREQUENCY DRINK/DRIVE BY AWARE MADD BY AGE

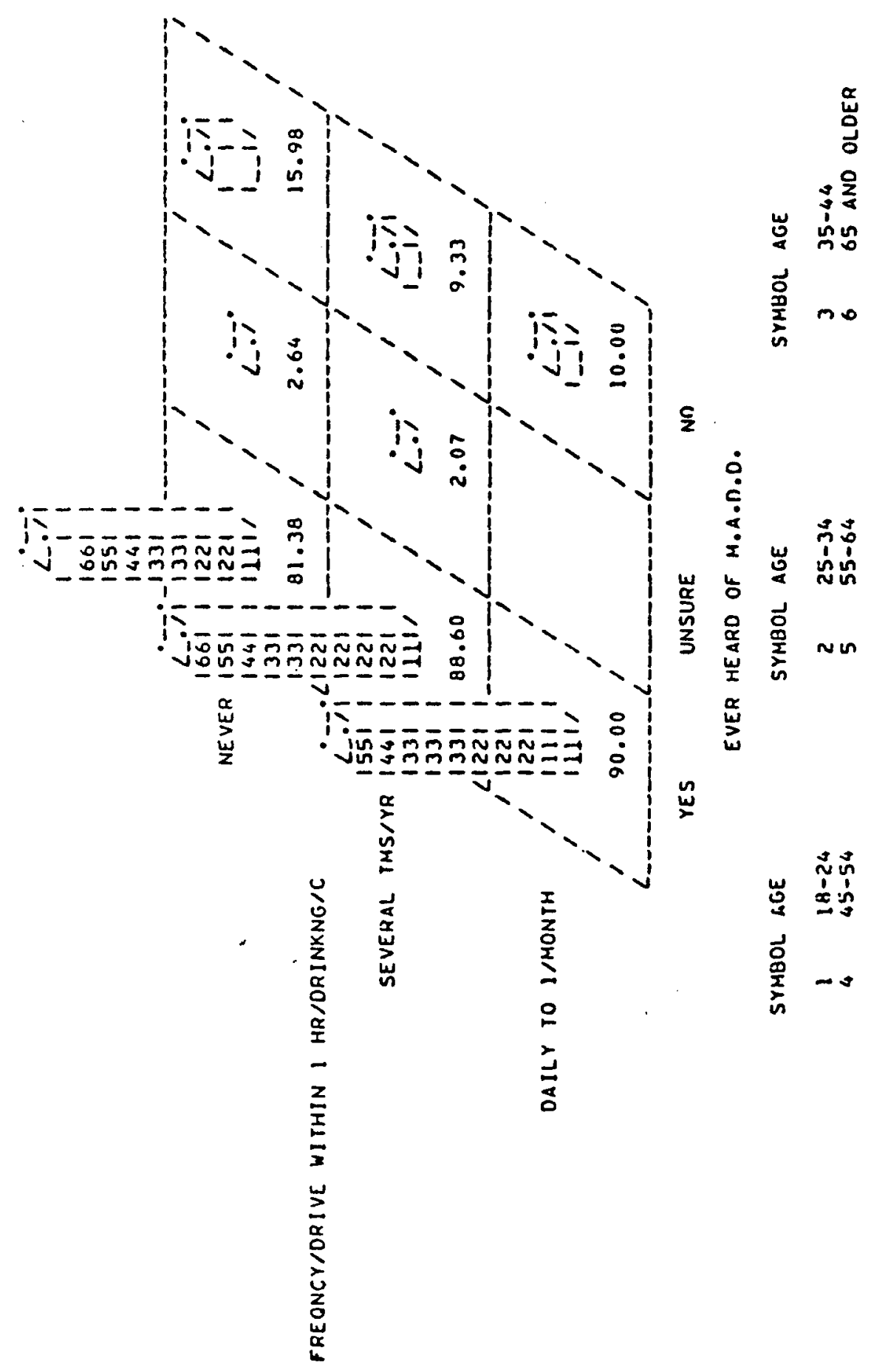


TABLE 49 - DEMOGRAPHICS BY AWARE OF MADD

TARRANCE \& ASSC. - SAFETY BELT (MOOD) - \#2017

PLQ/EVER HEARD OF M.A.O.D.

YES UNSURE NO TOTAL

STANDAFD FED. ADMNSTRATV REGIONS

$\begin{array}{lllrr}\text { I/NEW ENGLNO STS } & 92.59 & 1.85 & 5.56 & 54 \\ \text { II/NW YRK/NW JRY } & 82.14 & 1.79 & 16.07 & 112 \\ \text { III/MIO ATLANTIC } & 91.43 & 0.95 & 7.62 & 105 \\ \text { IV/SOUTH EAST } & 77.59 & 5.17 & 17.24 & 174 \\ \text { V/GREAT LAKES } & 82.76 & 2.46 & 14.78 & 203 \\ \text { VI/MID SOUTH } & 81.25 & 0.00 & 18.75 & 112 \\ \text { VII/PLAINS } & 73.58 & 1.89 & 24.53 & 53 \\ \text { VIII/NW PLAINS } & 93.75 & 3.13 & 3.13 & 32 \\ \text { IX/WEST COAST } & 92.56 & 0.00 & 7.44 & 121 \\ \text { XINORTH WEST } & 94.12 & 0.00 & 5.88 & 34\end{array}$

RESPONDENT'S AGE

$\begin{array}{lllll}18-24 & 81.34 & 0.75 & 17.91 & 134 \\ 25-44 & 88.21 & 1.26 & 10.53 & 475 \\ 45 \text { AND OVER } & 81.07 & 3.32 & 15.50 & 391\end{array}$

RESPONDENTIS EDUCATION

$\begin{array}{lllll}\text { LT HIGH SCHOOL } & 72.32 & 2.68 & 25.00 & 112 \\ \text { HIGH SCHOOL GRAO } & 84.91 & 1.89 & 13.21 & 371 \\ \text { SOME COLLEGE } & 84.81 & 2.59 & 12.59 & 270 \\ \text { COLLEGE GRAD } & 89.07 & 1.21 & 9.72 & 247\end{array}$

RESPONDENT'S SEX

$\begin{array}{lllll}\text { MALE } & 80.96 & 2.81 & 16.23 & 499 \\ \text { FEMALE/HOME } & 87.65 & 0.82 & 11.52 & 243 \\ \text { FEMALE/EMPLOYED } & 88.37 & 1.55 & 10.08 & 258\end{array}$

PRIMARY SOURCE OF FAMILY INCOME/C

$\begin{array}{llllr}\text { SLF-EMPL/WHITE C } & 85.81 & 2.33 & 11.86 & 430 \\ \text { BLUE-COLLAR } & 86.01 & 1.23 & 12.76 & 243 \\ \text { NOT EMPLOYED } & 78.40 & 2.82 & 18.78 & 213 \\ \text { OTHER } & 87.72 & 0.88 & 11.40 & 114 \\ \text { AGGREGATE } & 84.50 & 2.00 & 13.50 & 1000\end{array}$


TABLE 49 (continued)

TARRANCE \& ASSC. - SAFETY BELT (MOOD) - \#2017

$$
\begin{aligned}
& \text { P49/EVER HEARJ OF M.A.0.0. } \\
& \text { YES UNSURE NO TOTAL }
\end{aligned}
$$

\begin{tabular}{|c|c|c|c|c|}
\hline NOT APOLICABLE & & & & \\
\hline FREQ USERS/CNFRM & 86.82 & 1.61 & 11.58 & 311 \\
\hline SMTM USER儿IKELY & 86.15 & 2.16 & 11.59 & 231 \\
\hline SMTM USER/UNLKLY & 76.09 & 0.00 & 23.91 & 92 \\
\hline INFREO USR/LIKLY & 85.90 & 2.98 & 10.12 & 168 \\
\hline INFREO USR/CNFRM & 82.12 & 2.55 & 15.23 & 151 \\
\hline 65/WHERE DO YOU & & & & \\
\hline IN $A$ BIG CITY & 82.01 & 2.65 & 15.34 & 189 \\
\hline SUBURES/RIG CITY & 86.41 & 1.74 & 11.85 & \\
\hline IN A SIALLL CITY & 84.38 & 1.79 & 13.84 & 224 \\
\hline TOWNN VILLAGE & 84.12 & 0.59 & 15.29 & 170 \\
\hline IN THE COUNTRY & 85.04 & 3.15 & 11.81 & 127 \\
\hline OKINO ANSWER & 66.67 & 33.33 & 0.00 & 3 \\
\hline FREZ-OOV-1 HR ORN & & & & \\
\hline OAILY TO LMONTH & 90.00 & 0.00 & 10.00 & 200 \\
\hline SEVERAL TMS/YR & 88.60 & 2.07 & 9.33 & 193 \\
\hline NEVER & & 2.64 & 15.98 & 607 \\
\hline$\triangle A G R E G A T E$ & 84.50 & 2.00 & 13.50 & 1000 \\
\hline
\end{tabular}

USER CATAGORIES 
TABILE 50

CODES - WHAT IS MADD

And can you tell me what MADD is or what they are trying to do?

01. PREVENT DRUNKEN DRIVING - trying to keep hard-core drinkers off the road/trying to get drunk drivers off the road P.T.A. is for them/prevent drunk drivers to keep them off the street/trying to stop drunk drivers from getting back on the road/trying to get drunks off the highway/

02. WANT STRONG LAWS - organization trying to strengthen drunk driving laws/to get legislation passed to get drunk drivers off the road/to get better laws against drunk driving/ their objective is to get more laws passed to be harder on drunk drivers/a group of mothers getting together trying to get stiffer legislation against drunk drivers/

03. WANT SEVERE PENALTIES - they are trying to get stricter penalties against drivers convicted of manslaughter due to drunk driving/they are trying to get stiffer.fines and jail sentences for drunk drivers/the people involved are getting more severe punishment for drunk drivers/ they need stronger punishments/

04. PERSONALLY AFFECTED/ORUNK DRIVERS - an organization started by a woman whose son was killed by a drunk driver/they all have had a child killed in a car accident/a group started because children were killed by drunk drivers/parents of maimed children/mothers who have had their children killed by drunk drivers, and they try to console other victims/

05. REDUCE DRINKING-RELATED DEATHS/ACCIDENTS - prevent people driving when drunk and people getting killed/trying to keep drunk drivers off the road so that they will not kill the children/trying to decrease the number of highway deaths/trying to cut down on drunk driving deaths/ lower number of accidents/

06. STRICTER ENFORCEMENT - trying to enforce strict laws against D.W.I./stricter enforcement of drunk driving laws/trying to convict drunk drivers/

07. STOP DRINKERS FROM DRIVING - trying to keep people who drive off the roads/trying to put a stop to folks driving and drinking/they formed a group to keep people from drinking and driving/attempting to get the drinkers out from behind the wheel of a carl 
TABLE 50 (continued)

08. AGAINST DRUNK DRIVERS - their efforts are against drunk drivers/control drunk drivers in this nation/getting the state to crack down on drunk drivers/women striking back at drunk drivers/against drunk drivers/complaining about drunk driving/angry at drunk drivers/

09. EDUCATE PUBLIC - to make people more aware of drunk,drivers/ trying to deve 1 op a groundswell against drunk driving/ they are trying to bring the public some education/make drunk drivers more aware of the punishments they will receive/educate young people not to drink and drive/

10. JAIL SENTENCES FOR D.W.I. - they are trying to get mandatory jail sentences for drunk drivers/put drunk drivers in jail for 48 hours and if they have had an accident and killed...put them in prison/

11. SAW MOVIE/PUBLICITY - I saw a movie about them a few weeks ago/all I know is what I saw on T.V. about them/ seen a headline in the newspaper about that/I've just heard of them/

12. RAISE DRINKING AGE/STOP TEEN DRUNKS - enforcing laws for teens not to drive during or after drinking/keep kids under 25 from drinking/get those kids off the road/ they are trying to have the drinking age raised/

13. OTHER

14. DON'T KNOW/NO ANSWER 
TABLE 51 - DEMOGRAPHICS BY IS MADD SUCCEEDING

TARKANCE \& ASSC. - SAFETY BELT (MOOD) - \#2017

$$
\begin{aligned}
& \text { P502/0O YOU THINK M.A.0.0. IS SUCCEEDIN } \\
& \text { NOT APPL } \\
& \text { ICABLE }
\end{aligned}
$$

TOTAL

STANDARD FED. AOMNSTRATV REGIONS

I/NEW ENGLND STS
II/NW YRK/NW JRY
III/MID ATLANTIC
IV/SCUTH EAST
V/GREAT LAKES
VI/MIO SOUTH
VII/PLAINS
VIII/NW PLAINS
IX/WEST COAST
X/NORTH WEST

RESPONOENT'S AGE

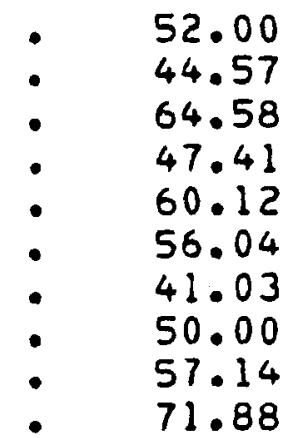

62.39
$: \quad 54.17$
$: \quad 54.19$
$: \quad 59.26$
$: \quad 46.79$
$: \quad 53.00$

RESPONDENT'S EDUCATION

LT HIGH SCHOOL

HIGH SCHOOL GRAO

SOME COLLEGE

COLLEGE GRAD

RESPONDENTIS SEX

MALE

FEMALE/HOME

FEMALE/EMPLOYED

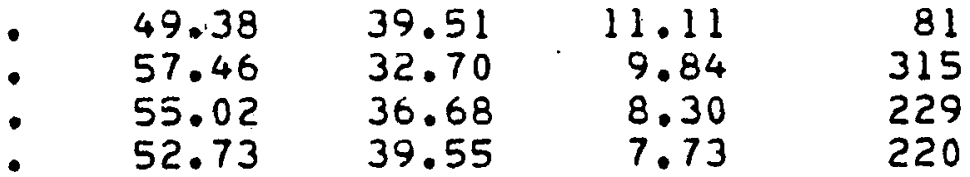

36.00

12.00

50

42.39

13.04

10.42

8.89

7.74

7.69

5.13

6.67

10.71

0.00

28.13

29.36

39.17

8.26

109

35.20

6.67

240

33.33

10.61

7.41

179

108

38.53

14.68

109

39.00

8.00

100

168

91

39

112

32

96

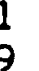
5 
TABLE 51 (continued)

TARRANCE \& ASSC. - SAFETY BELT (MOOO) - \#2017.

PSO2/0O YOU THINK M.A.O.0. IS SUCCEEDIN
NOT APPL
ICABLE

USER CATAGORIES

NOT $\triangle P P L I C A B L E$

FREO USERS/CNFRM

SMTM USER/LIKELY

SMTM USER/UNLKLY

INFREQ USR/LIKLY

INFREQ USR/CNFRM

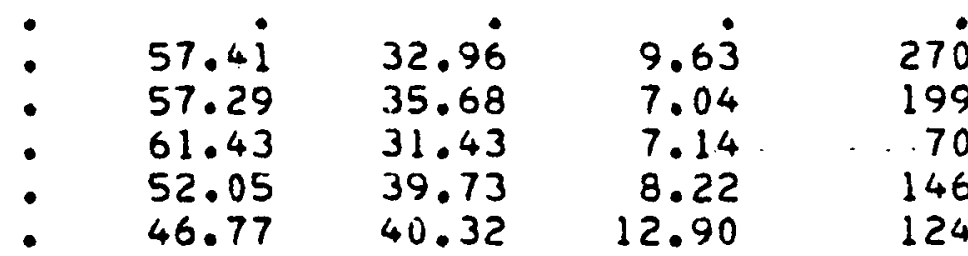

P65/WHERE DO YOU LIVE

IN A BIG CITY

SUBURBS/BIG CITY

IN A SMALL CITY

TOWN/VILLAGE

IN THE COUNTRY

OK/NO ANSWER

FREQ-ORV-1 HR DRNKNG

DAILY TO I/MONTH

SEVERAL TMS/YR

NEVER

AGGREGATE

$\begin{array}{rrrrr}: & 49.68 & 43.87 & 6.45 & 155 \\ : & 56.05 & 33.47 & 10.48 & 248 \\ : \quad 52.91 & 37.57 & 9.52 & 189 \\ : \quad 55.94 & 33.57 & 10.49 & 143 \\ : \quad 61.11 & 32.41 & 6.48 & 108 \\ & 50.00 & 50.00 & 0.00 & 2\end{array}$

\begin{tabular}{|c|c|c|}
\hline 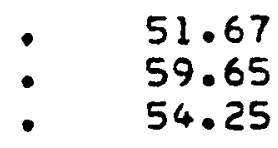 & $\begin{array}{l}37.22 \\
35.09 \\
36.23\end{array}$ & $\begin{array}{r}11.11 \\
5.26 \\
9.51\end{array}$ \\
\hline 54.79 & 36.21 & 8.99 \\
\hline
\end{tabular}


And in your own words, what are one or two reasons why you feel that way?

01. NEW/STRICTER LAWS - passed new drunk driving laws/laws in (20\%) Colorado have gotten stricter partly due to this organization/just passed tougher laws here/we've had tougher drunk driving laws passed in the past year/

02. PUBLICITY - they get lots of publicity here/in advertising/ from hearing a lot about them on the news/hear more about $i t /$ they've gotten more publicity/they are getting more publicity than before/

03. PUBLIC AWARENESS - bringing awareness to the problem/ people are paying more attention to the problem/people are becoming more aware of the dangers of drunk drivers/ more people are aware of the problem/

04. STIFFER/INCREASED PENALTIES - in New York state there's a real push to get stiffer penalties/forcing the states to pass stiffer legislation against drunk drivers/drunk drivers are getting tougher sentences and more fines/ you get jailed for the first drunk driving offense now in Pennsyivanial

05. TELEVISION PRESENTATION - I happened to see their televised drama last week/I watched it on TV and I think they have expanded/I saw the movie last week/through the media/more known, especially on the medial

06. ENFORCING D.W.I. LAWS - police are really enforcing drunk driving laws/more arrests and convictions are being made/ in Seattle they are really cracking down on anyone caught drinking and driving/

07. WHAT I'VE SEEN/READ/HEARD - I'm hearing on news and in the paper/more publicity in newspapers/they're introducing their program to the media/from what I've read they've been successful/

08. RECOGNITION OF ORGANIZATION - recognition/they are succeeding because they are getting a lot of attention/getting more recognition now/

09. PERSONAL EXPERIENCES - because of my own experience/she had a friend lose her Tife because of drinking/there's been drunk drivers in my neighborhood/we were hit by a drunk/ we had a few kids killed by drunk drivers in this area/ 
TABLE 52 (continued)

10. GROWING/EFFECTIVE - more public involvement/they are doing what they set out to do/more and more people becoming involved in this issue/they put on a good campaign/they've got more people joining now/

11. FEWER ORUNKEN DRIVERS/ACCIDENTS - number of accidents due to alcohol has dropped/looking at the obituaries you can tell it's much less/less people on the road that are drunk/less accidents because of drunk drivers/getting many off the streets/

12. DOING A GOOD JOB - they are getting the message across/ it's very valuable, accomplish a lot/strong lobby and determination of them/people are paying more attention to the problems/it is a good cause that people are willing to support/

13. DON'T LIKE DRUNK DRIVERS - they've got to crack down on drunk drivers/don't think drunk drivers should be on the $\mathrm{road} /$ too many drinking drivers/drunk: drivers hurt innocent bystanders than they hurt themselves/

14. NEED STRONGER PUNISHMENT - it's better but the punishment isn't hard enough stil17people are still getting away with drunk driving and killing people without a harsh punishment/some just get away scot-free/

15. NEED EDUCATION - "any" education in the public the better the chances are of getting results to the problem/good way to run them of $f$ the road - by educating others of the problem/educating lot of people/a lot of people who drink and drive are educated and through education they realize not to drive and drink/

16. PARENTAL REACTION - concern for their kids/lots of mothers and fathers who are complaining about drunk drivers now/ I've got children and would hate to see them get hit by a drunk driver/the mothers are upset about their kids and relatives being injured and killed/

17. NEED MORE TIME/SCOPE - it has helped but they need more time/they are not reaching enough to many judges and police/hasn't been a major change so far but their future looks hopeful/still have a long way to go/

18. NEEDLESS DEATHS/ACCIDENTS - it will stop deaths/seeing wrecks with young children involved/if they take someone's life they should be punished for it/they're just killing a lot of innocent people/

19. PROBLEM DRINKING - alcoholism is a problem/our society drinks too much 7 don't believe in drinking/ 
TABLE 52 (continued)

20. TRY HARDER/GET INVOLVED - feel that people should try harder/if they can do something about drunk drivers they should at least try/not enough people involved/

21. TAKING A STAND - people are speaking up for what's going now/I hear peer groups are concerned about drunken driving by talking about it/

22. LEGISLATIVE ALLIES - they are complaining to the law about the drunk drivers/we have some bills in town and legislature - they will probably get passed/

23. BENEFITS ALL - it might make driving safer/it benefits everyone/we11 safety of all of us/

24. MAKING AN IMPRESSION - it seems like it's hitting home/ children seem to be getting the message/you can tell the difference between the ones who are driving now than one year ago/.

25. SERIOUS NATTER - it's such a serious problem/it's a very serious tning and people listen to it/don't see how you can live with it if you killed a child/

26. OTHER

27. DON'T KNOW/NO ANSWER 
Table 50 provides examples of the responses given to the question "What is MADD trying to do", and the percentage of the respondents who gave those response. A number of the reasons given clearly indicates that the respondents did, in fact, know what MADD was and what they were trying to accomplish. When asked the question about whether MADD was succeeding, $55 \%$ of the respondents said "yes". The reasons they gave for feeling that way are presented in Table 52. The response sets to both of these questions strongly suggest that MADD has in fact become a cultural change agent with regard to the drunk driving problem. 
TABLE 53

\section{VERBATIMS - WHAT IS MADD}

Question 50-1:

And can you tell me what MADD is or what they are trying to do?

\begin{tabular}{lllll}
\hline $\begin{array}{l}\text { Geographic } \\
\text { Region }\end{array}$ & Age & Education & Sex & $\begin{array}{l}\text { User } \\
\text { Categories }\end{array}$ \\
\hline $\begin{array}{l}\text { I/New England } \\
\text { States }\end{array}$ & $35-44$ & $\begin{array}{l}\text { College } \\
\text { Graduate }\end{array}$ & Male & $\begin{array}{l}\text { Infrequent/ } \\
\text { Likely }\end{array}$
\end{tabular}

"Trying to obviously stop drunk'drivers... I'm at the moment involved with a case about MADD... one of my students had just a little too much to drink and he hit a girl...he is not the type of guy to really do that, it was just a freak accident that it happened to him."

V/

Great Lakes
High School

Graduate
Infrequent/

Likely

"A group of mothers whose children were victims of drunk drivers... how they're trying to get stiffer laws and penalties for the drunk drivers...I think they are also trying to get the drinking age changed in some places."

V/

Great Lakes

$55-64$

High School

Graduate
Female/

Home
Sometime/

Likely

"Mothers Against Drunk Drivers...they are trying to get laws passed to get drivers off the road... I certainly agree with them...I think the current laws should be stricter."

II/Nlew Yorik/

New! Jersey

25-34 Gollege

Male

Frequent/

Confirmed

"Trying to get fines and penalties increased heavily and to give public awareness campaigns to show how drunk driving damages society."

V/

Great Lakes
College

Graduate
Male Infrequent/

"It is mothers who have had their children killed by drunk drivers and they try to console other victims... they also try to change laws."

VII/ Plains
High School Graduate
Female/ Works
Sometime/

Unlikely

"Started by a woman whose young child was killed by a drunk driver and they are involved in getting stricter penalties and laws against drunk drivers." 
TABLE 53 (continued)

Question 50-1 continued:

\begin{tabular}{lllll}
\hline $\begin{array}{l}\text { Geographic } \\
\text { Region }\end{array}$ & Age & Education & Sex & $\begin{array}{l}\text { Jser } \\
\text { Categories }\end{array}$ \\
\hline IV/ & & $\begin{array}{l}\text { High School } \\
\text { Graduate }\end{array}$ & Male & $\begin{array}{l}\text { Sometime/ } \\
\text { Likely }\end{array}$
\end{tabular}

"A group that is trying to protect their children by getting the drunk drivers off the road... they want stiffer penalties for drunk drivers...they also want the drivers to be responsible for their actions while driving."

IXI

West coast

College

$25-34$

Graduate

Female/ Works

Sometime/

"Trying to get press coverage to heighten public awareness to the tragedies of drunk driving caused by repeated offenders...they are trying to legislate tougher laws to get this treated like homicide and get the repeated offenders locked up behind bars."

IXI

West coast

45-54

Some

Female/

Sometime/

College

Works

Unlikely

"Trying to get a law passed that has a mandatory jail sentence for drunk drivers."

VI/

Mid South

$35-44$

High School

Graduate

Mare

Infrequent/

Likely

"It is Mothers Against Drunk Drivers and they are attempting to get the drinkers out from behind the wheels of cars." 
TABLE 54

VERBATIMS - WHY YOU THINK MADD IS SUCCEEDING

Question 51:

And in your own words, what are one or two reasons why you feel that Mothers Against Drunk Drivers is succeeding?

\begin{tabular}{|c|c|c|c|c|}
\hline $\begin{array}{l}\text { Geographic } \\
\text { Region }\end{array}$ & Age & Education & Sex & $\begin{array}{l}\text { User } \\
\text { Categories }\end{array}$ \\
\hline $\begin{array}{l}\text { VII/ } \\
\text { Plains }\end{array}$ & $\begin{array}{l}65 \& \\
\text { Over }\end{array}$ & $\begin{array}{l}\text { Some } \\
\text { College }\end{array}$ & $\begin{array}{l}\text { Female/ } \\
\text { Home }\end{array}$ & $\begin{array}{l}\text { Frequent/ } \\
\text { Confirmed }\end{array}$ \\
\hline
\end{tabular}

"The children seem to be getting the message...they're not driving after they drink."

VI/

Mid South
High. School

Graduate
Female/ Infrequent/

Home Likely

"They have gotten some states drinking and driving laws changed and they've brought it out in the open and made the public aware of how big a problem it is."

I/New England

States

\section{$45-54$}

High School

Graduate
Sometime/

Male Unlikely

"They're making an impact...I work for a newspaper and I see that they're getting a lot of ink lately and that's good."

$\begin{array}{llll}\text { I/New England } & \begin{array}{l}\text { High School } \\ \text { Graduate }\end{array} & \begin{array}{l}\text { Female/ } \\ \text { Works }\end{array} & \begin{array}{l}\text { Infrequent/ } \\ \text { Confirmed }\end{array} \\ \text { States } & 55-64 & \text { Wratal }\end{array}$

"People are becoming more aware of the dangers of drunk driving and people are letting the police know that they want drunk drivers off the road."

IV/

South East
Less than

High School
Female/

Works
Infrequent/

Likely

"I watched the show on TV the other night and I got the impression they were accomplishing what they set out to do."

VI/

Mid South
High Schoor

$25-34$

Graduate

Female/

'Frequent/ Works Confirmed

"Recognition and word of what they're doing is noticeable...they have support because of the problem." 
TABLE 54 (continued)

Question 51 continued:

\begin{tabular}{|c|c|c|c|c|}
\hline $\begin{array}{l}\text { Geographic } \\
\text { Region }\end{array}$ & Age & Education & Sex & $\begin{array}{l}\text { User } \\
\text { Categories }\end{array}$ \\
\hline $\begin{array}{l}\text { VIII/ } \\
\text { New Plains }\end{array}$ & $25-34$ & $\begin{array}{l}\text { High School } \\
\text { Graduate }\end{array}$ & $\begin{array}{l}\text { Female/ } \\
\text { Works }\end{array}$ & $\begin{array}{l}\text { Infrequent } \\
\text { Confirmed }\end{array}$ \\
\hline
\end{tabular}

"Because they are people personally involved and others will be more likely to Tisten to them."

$x /$

North West

$35-44$

College

Graduate
Sometime/

Male Unlikely

"They are building momentum...this particular state legislature is dealing with this now...entities dealing with this now are becoming more successful due to public awareness."

$x /$

North West
College

Graduate
Sometime/

Male

Unlikely

"Because the cause they are supporting is right...they go about it in a logical way with lobbying and public support."

IV/

South East
College

Graduate
Sometime/

Likely

"Drunk driving is unnecessary...presently the law is not strict enough against drunk drivers... it is negligent to drive intoxicated...life is very precious... we need to keep life." 
APPENDIX A

INTERVIEWER

TARRANCE \& ASSOCIATES

PERSONAL/CONFIDENTIAL
STUDY \#

TIME STA TTED

TIME ENDED

FINANCE

CODING

Hello, I'm

of Tarrance \& Associates, a national research firm. We re talking to people about topics relating to automobile safety.

A. Are you 18 years of age or older?

IF "NO", ASK:
home that is 18? there anyone
(REPEAT SCREEN)

Yes (CONTINUE) ................

No (THANK \& TERMINATE) .......

As you may know, there are many different things we can do to increase auto safety and decrease the number of injuries and deaths which result from auto accidents. Which of the following do you think would be most important in making driving safer? (READ LIST, ROTATE) And which do you think would be the second most important?

1. Less people drinking and driving

2. More people wearing seat belts

3. Requiring motorcyclists to wear helmets

4. Stricter enforcement of traffic Jaws

5. Safer cars

6. Don't know/no answer
Q.1

Most

Important

Q. 2

2nd Most

Important

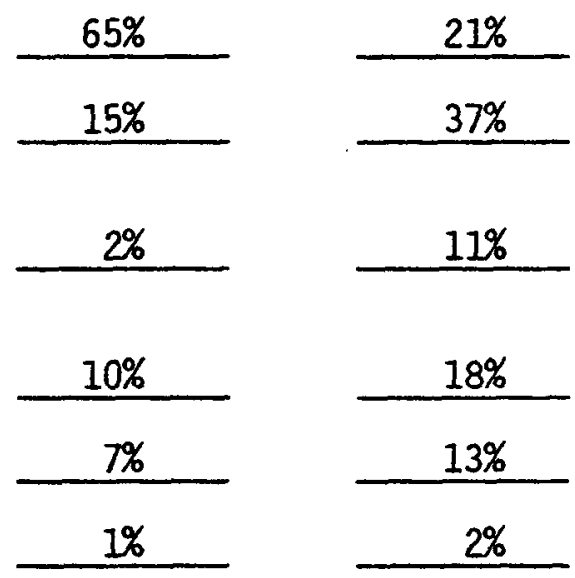


Now I'd like to get your opinions on several different issues relating to seat beits. First --

3. How often do you wear seat beits when roads are wet, or snow and ice covered? (READ ALTERNATIVES)

Aiways......................

Most of the time.............

Sometimes..................

Never.....................

Don't know/no answer..........

4. How often do you wear seat belts on

long trips? (READ ALTERNATIVES)

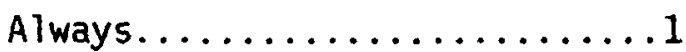

Most of the time............2

Sometimes................. 3

$(16 \%)$

Never. ...................4

(34\%)

Don't know/no answer......... 5

5. How often do you wear seat belts on short trips around town? (READ ALTERNATIVES)

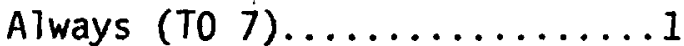

Most of the time (TO 6 ).......

Sometimes (TO 6).............

Never (TO 6)..............4

IF "MOST OF THE TIME", "SOMETIMES", OR "NEVER" IN 0.5, ASK:

Do you agree or disagree with the following statement --

6. "There's nothing anyone can do that would make me use seat belts all of the time."

IF CHOICE MADE, ASK:
do you feel strongly about
that?

Agree/strongly..............

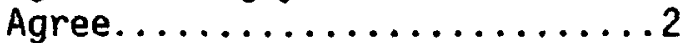

Unsure (DO NOT READ) ..........

Disagree.................

Disagree/strongly...........

And do you agree or disagree with the following statements --

7. "I'm quite sure I won't be in an auto accident because I drive defensively." (PROMPT: DO YOU AGREE OR DISAGREE WITH THIS STATEMENT?)

IF CHOICE MADE, ASK: And do you feel strongly about that?
Agree/strongly............

Agree.....................

Unsure (DO NOT READ) .........

Disagree.................4

Disagree/strongly..........5 
8. "Wearing seat belts is like many other good health practices such as brushing your teeth and having regular medical check-ups."

(PROMPT: DO YOU AGREE OR DISAGREE WITH THIS STATEMENT?)

IF CHOICE MADE, ASK: And

Agree/strongly.............

do you feel strong Ty about. Agree...................

Unsure (DO NOT READ) ......... that?

Disagree.................4

Disagree/strongly..........5

9. "People think they won't be in an accident because they are safe drivers in control of their car." (PROMPT IF NECESSARY)

IF CHOICE MADE, ASK: And do you feel strongly about

Agree/strongly............

Agree. . . . . . . . . . . . . . . 2 that?

Unsure (DO NOT READ) .........

Disagree. ...............

Disagree/strongly.........5

10. "People are more likely to put their seat beits on if they think that driving conditions are more dangerous than usual."

(PROMPT IF NECESSARY)

IF CHOICE MADE, ASK:
do you feel strongly about
that?

Agree/strongly.............

Agree.................... 2

Unsure (DO NOT READ) ......... 3

Disagree................. 4

Disagree/strongly......... 5

11. "I don't think seat belts work properly when they feel so loose around me when I fasten them." (PROMPT IF NECESSARY)

IF CHOICE MAOE, ASK:
do you feel strongly about
that?

Agree/strongly............

Agree...................

Unsure (DO NOT READ)........

Disagree................. 4

Disagree/strongly.......... 5 
Now I'd like to read you some statements about seat belts made by two people. Please tell me which one comes closest to your own cpinion --

12. Mr. Jones feels that he can fulfill his responsibility to passengers in his car by asking them to fasten their seat belts and by driving carefully.

Mr. Smith feels that he can fulfill his responsibility to passengers in his car by making sure that they are covered under his auto insurance policy.

Who comes closest to your own opinion

-- Mr. Jones or Mr. Smith?

$\frac{\text { If CHOICE MADE, ASK: }}{\text { you feel strongly about that? }}$

Mr. Jones/strongly...........

Mr. Jones....................

Unsure (DO NOT READ)..........

Mr. Smith..................

Mr. Smith/strongly..........

13. Mr. Jones feels that he can fulfill his responsibility to passengers in his car by asking them to fasten their seat belts and by driving carefully.

Mr. Smith never asks passengers in his car to fasten their seat belts because he feels he would be imposing on them if he did so.

Who comes closest to your own opinion

-- Mr. Jones or Mr. Smith?

IF CHOICE MADE, ASK:
you feel strongly about that?

Mr. Jones/strongly...........

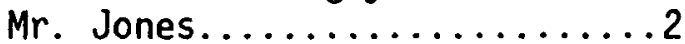

Unsure (DO NOT READ) ..........

Mr. Smith.................4

Mr. Smith/strongly..........

14. Mr. Jones feels that the car's buzzer is a reminder to him to put on his seat belt.

Mr. Gray on the other hand just ignores the buzzer when he gets into his car and doesn't put his belt on.

Who comes closest to your own opinion

-- Mr. Jones or Mr. Gray?

$\frac{\text { IF CHOICE MADE, ASK: }}{\text { you feel strongly about that? }}$

Mr. Jones/strongly...........

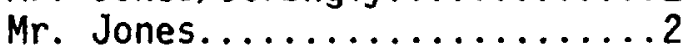

Unsure (DO NOT READ) ..........

Mr. Gray.................4

Mr. Gray/strongly...........5 
15. Mr. Jones thinks that if he were in a serious auto accident and were using a safety belt his injuries would be less than if he wasn't wearing a safety belt.

Mr. Smith on the other hand feels that if he were in a serious auto accident and was wearing a safety belt his injuries would be more severe, therefore he doesn't wear his safety belt.

Who comes closest to your own opinion -- Mr. Jones or Mr. Smith?

IF CHOICE MADE, ASK:
you feel strongly about that?

Mr. Jones/strongly..........1

Mr. Jones..................

Unsure (DO NOT READ)...........

Mr. Smith..................

Mr. Smith/strongly..........

On another matter --

16. Mr. Johnson feeis that it takes too much time and is too hard to remember to fasten his seat belt every time he sits down in his car, so he rarely uses them.

Mr. Davis feels that putting on seat belts is just like brushing his teeth or watching his diet, and it is worth the slight inconvenience it takes to fasten his seat belts every time he sits down in his car.

Who comes closest to your own opinion -- Mr. Johnson or Mr. Davis?

IF CHOICE MADE, ASK:
you feel strongly about that?

Mr. Johnson/strongly..........

Mr. Johnson..................

Unsure (DO NOT READ) ...........

Mr. Davis.................

Mr. Davis/strongly..........

17. Mr. Cooper does not wear seat beits because he thinks he is a good driver and won't be in an accident.

$M r$. Lewis wears his seat belts because he isn't sure about the other drivers and just doesn't like to take chances.

Who comes closest to your own opinion

-- Mr. Cooper or Mr. Lewis?

IF CHOICE MADE, ASK:
you feel strongly about that?

Mr. Cooper/strongly..........1

Mr. Cooper..................

Unsure (DO, NOT READ) ...........

Mr. Lewis................ 4

Mr. Lewis/strongly........... 
And --

18. Mr. Thompson feels he has much less control over himself and his car when he drives with his seat belts on.

Mr. Evans feels he has much more control over himself and his car when he drives with his seat belts on.

Who comes closest to your own opinion

-- Mr. Thompson or Mr. Evans?

$\frac{\text { IF CHOICE MADE, ASK: }}{\text { you feel strongly about that? }}$

Mr. Thompson/strongly.........

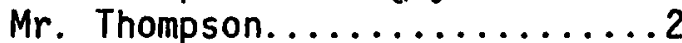

Unsure (DO NOT READ) .........

Mr. Evans................... 4

Mr. Evans/strongly.......... 5

On another matter --

19. How much contral do you feel you have in preventing your own involvement in automobile accidents? Do you feel you have... (READ ALTERNATIVES)

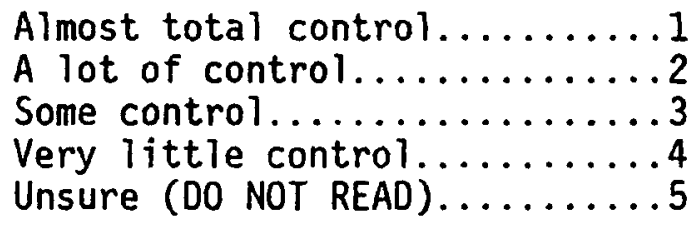

20. If you were involved in an auto accident, what do you feel would be the most likely cause? Would it be... (READ ALTERNATIVES)

1) Your own driving error $6 \%$

2) A drunk driver $21 \%$

3) Someone else's driving error $41 \%$

4) A mechanical defect in the car $3 \%$

5) Unavoidable bad luck $7 \%$

6) Poor road conditions $6 \%$

7) Poor weather conditions $14 \%$

8) Don't know/no answer $4 \%$ 
21. The total number of auto fatalities and the number of auto fatalities per mile driven dropped significantly last year. What do you think was the major cause for this drop? (INTERVIEWERS: DO NOT ACCEPT "DROVE LESS", "DIDN'T ORIVE AS MUCH." PROBE FOR OTHER ANSWERS)

01. LOWER SPEED LIMIT - just lowering the speed limit - that's the only reason/slower speed limit/lowering the speed limit did a lot of good/because of the drop in the speed limit/

02. 55 M.P.H. SPEED LIMIT - 55 m.p.h. limit is a good measure/reduction in speed from 65 to $55 /$ speed limits not going over $55 \mathrm{~m} . \mathrm{p.h} . /$ $55 \mathrm{~m} . \mathrm{p} . \mathrm{h}$. has cut down the death rate/55 m.p.h. speed limit has caused people to have less accidents/

03. DRUNK DRIVING LAWS - I would say the stricter alcohol laws/laws on drunk drivers are stricter here/the new alcohol laws against driving drunk/tougher drunk drivers laws/

04. CRACK DOWN ON DRUNK DRIVERS - cracking down on drunk drivers/ there is more of a watch on drunk drivers/cracking down on driving while drinking/more strict on drunk driving/

05. ENFORCEMENT OF DRUNK DRIVING LAWS - stricter enforcement on drunk drivers/enforcement of the D.W.I. laws/stronger police enforcement of drunk driving/

06. FEWER DRUNK DRIVERS/DRUGGED DRIVERS - there wasn't as many drunk drivers on the road/less drinking and driving/getting the drunk drivers off the road/people are smoking less pot/

07. PUBLIC CONCERN/DRUNKEN DRIVING - more talk about drinking and driving/an awareness of the public to drinking while driving/ people are cutting down on driving after having too much to drink/the crusade against drunken drivers/drunk driving groups, like M.A.D.D./

08. RAISING THE ORINKING AGE - raising the drinking age/taking liquor away from teens/many states raised the drinking age/raising the drinking age to 19/they raised the drinking age, that lowered the number of drunk drivers on the road/

09. SAFETY BELTS/CAR SEATS - people are using their seat belts more than they used to/I have been seeing a lot more people using their seat belts/because of seat belts/the news telling people to wear safety belts/infant car seats required by law/

10. MORE LAW ENFORCEMENT/ENFORCEMENT OF 55 M.P.H. LIMIT - there's been more law enforcement/police enforcement of the regulations/ starting to control traffic better/stricter enforcement of driving laws/enforcement of the speed 1 imit/cops are giving more tickets/tighter enforcement of the $55 \mathrm{~m} . \mathrm{p} . \mathrm{h}$. speed limit/

11. THE ECONOMY - with more people unemployed, they are unable to buy gas/economics, no money to drive/less people on the road now because of money/it costs more to get crashed cars fixed/ people don't have money to take trips anywhere/ 


\section{Question 21 continued}

12. PRICE OF GAS/GAS SHORTAGE - price of fuel, people can't afford to drive as much/people can't afford the gas/people are out of gas because of gas being so high/the lack of gas/people drove conservative to save gas/

13. REDUCED TRAFFIC/LESS DRIVING - less density if cars on the road/ people aren't driving as much because of the oil shortage/people are driving less/people driving less and putting less miles on the car/car pooling/

14. DRIVERS ARE CAREFUL - people drive more defensively/people are more careful and are safer drivers/people are becoming more alert/ people are more careful now because cars are becoming more expensive to fixl

15. PEOPLE ORIVE SLOWER - people driving slower speeds, more accident consciousness/people are driving a little bit slower/people travel at lower speeds/

16. PUBLIC AWARENESS/EDUCATION - people taking defensive driving/better education of drivers/a lot of national publicity about safer driving/people benefit from hearing about accidents/more awareness of driving habits/people are more aware of safety/more awareness of driving hazards/public awareness to accidents has improved significantly/

17. SAFER CARS - making the automobile safer/smaller engines in the cars/concentration on safety in cars/cars have been built safer/ safer cars, there is a lot less old junk on the roads/all the new cars coming in with new automatic safety belts that flip on with the door/

18. STRICTER LAWS - stricter traffic laws/tough laws/stricter laws being imposed/pushing more safety regulations/the new law/tougher laws about speeding and so forth/more speeding laws slowing up trucks/stiffer penalties/

19. WEATHER/ROAD CONDITIONS - we had a milder winter/not as much ice and snow/road conditions/bad roads, lower speed/snow falling more and less people driving/

20. MORE POLICE ON THE ROADS - definitely the increase in the police/ more patrols on the highway/more police out stopping reckless and drunken drivers/ 21. INSURANCE REQUIREMENTS - insurance requirements/the large demand

22. OTHER

23. DON'T KNOW/NO ANSWER 
22. Do you drink alcoholic beverages -even occasionally -- or are you a total abstainer?

Yes (CONTINUE) $\ldots \ldots \ldots \ldots \ldots 1$

Total abstainer (TO 34).......2

23. Think for a moment about your own personal driving habits over the past year. Trying to be as honest as you can, how frequently would you say you drive within one hour of drinking an alcoholic beverage? (READ ALTERNATIVES)

Daily....................

2 to 6 times a week...........2

Once a week...............

Once every two weeks.........4

Once a month.............

About 4-6 times a year.......6 6

About once or twice a year.....7

Never (ASK 24 ONLY, THEN

SKIP TO 33)............ 8

Don't know/no answer.........9

24. And, one year ago, how frequently would you say you drove within one hour of drinking an alcoholic beverage? (READ RESPONSES)

Daily.....................

2 to 6 times a week..........2

Once a week................

Once every two weeks.........4

Once a month...............5

About 4-6 times a year......6

About once or twice a year....7

Never..................8

Don't know/no answer........ 9

25. And would you say your frequency of drinking within one hour of driving has changed significantly over the past year?

Yes (TO 26)...............

Unsure (TO 27)..............

No $($ TO 27$) \ldots \ldots \ldots \ldots \ldots \ldots . \ldots 3$ 
IF "YES" IN QUESTION 25, ASK:

26. And what are one or two reasons why you feel that way?

(PROBE: PLEASE TELL ME MORE ABOUT THAT)

01. STRICTER D.W.I. LAWS - our stiffer drunk driving laws/ laws are tougher/the new D.W.I. law that enforces 48 hours in jail/they are cracking down on laws for drunk drivers/

02. DON'T DRINK AS MUCH - I slowed down a lot on my drinking/ I have cut down a lot on my drinking/I consume much less alcohol/I am drinking less than I used to/

03. AM OLDER/MORE MATURE - I'm just getting oider and thinking more about it/getting older, more cautious/as you get a little older, you slow down/I am older and more mature/

04. MOZE FESPONSIBILITY - more responsibility, I am thinking of the safety of myself and my family/I am more safety conscious, I've got a child now/care about my kids/

05. DON'T GO OUT AS MUCH - I don't go out as much, I usually drink at home/I don't go much anymore/married and didn't party as much/we don't go out very often/my social activities have become less/

06. MORE AWARE - I am more aware of the effects of drinking and driving/I'm more aware of it being dangerous/awareness of drunk drivers/

07. GREATER DANGER OF ACCIDENTS - accidents I've heard about/ accidents around me/I realize the greater danger of accidents and I've become a better driver/more aware of accidents relating to drunk drivers/

08. DON'T DRINK AND DRIVE - don't drink and drive that much/I don't believe in drinking and driving/don't drink as often just before driving/I just have my wife drive me/I try not to drink when I'm driving/

09. AFRAID/DON'T FEEL SAFE - I'm afraid/drunken drivers scare me/don't feel safe anymore/

10. HAVE BEEN CAUGHT - got caught drinking and driving/I don't have a license and I have been arrested for drunken driving/I had a close call/

11. MONEY - my income has changed significantly/money/I can't afford to go out to clubs or parties as much anymore/

12. MEDICAL REASONS - I've got medical problems... the doctor told me not to drink/I didn't drink last year because I was pregnant/ 
Question 26 continued

13. PERSONAL/BUSINESS REASONS - I've had a lot of personal probTems/I've got a steady girl friend and she tends to affect my judgment/if I take customers out or not, that makes it change

14. GO OUT MORE OFTEN - I go out more often/more social occasions/go to more social affairs and different functions/

15. DO IT MORE NOW - I do it more now/I've been drinking, lately/

16. LIFE STYLE - because of my life style/changed my life style/

17. SAFETY EDUCATION - safety education, films seen at work/

18. OTHER

19. DON'T KNOW/NO ANSWER

Now I'm going to list several places where people drink, and then occasionally find themselves having to drive home within an hour of having a drink. For each one, please tढ 17 ine how frequently you personally have had to drive hone from that place after drinking within one hour.

27a. Bars/taverns

PROMPT: HOW FREQUENTLY HAVE

YOU PERSONALLY HAD TO DRIVE

HOME FROM A BAR OR TAVERN AFTER

DRINKING WITHIN ONE HOUR --

More than once a week.........

About once a week...............

One or two times a month.......3

Less than once a month........4

One or two times a year.........

Never.................... 6

Don't know/no answer.........

\section{7b. Restaurants}

(PROMPT IF NECESSARY)

More than once a week.........1

About once a week............

One or two times a month.......3

Less than once a month........4

One or two times a year.......5

Never..................6 6

Don't know/no answer......... 7 
27c. Cocktail lounges

PROMPT: HOW FREQUENTLY HAVE YOU

PERSONALLY HAD TO DRIVE HOME FROM

A COCKTAIL LOUNGE AFTER DRINKING

WITHIN ONE HOUR --

More than once a week.........1

About once a week...........

Cne or two timies a month......3

Less than once a month.......4

One or two times a year.......5

Never..................6

Don't know/no answer.........7

27d. Other people's homes

(PROMPT IF NECESSARY)

More than once a week.........1

About once a week............

One or two times a month.......3

Less than once a month........4

One or two times a year.......5

Never..................6 6

Don't know/no answer..........

27e. Private party held in a public place, such as a hoted or banquet hall. (PROMPT IF NECESSARY)

More than once a week.........1

About once a week............2

One or two times a month......3

Less than once a month.......4

One or two times a year.......5

Never..................6 6

Don't know/no answer.........

27f. Sporting events

(PROMPT IF NECESSARY)

More than once a week.........1

About once a week............2

One or two times a month.......3

Less than once a month........4

One or two times a year.......5

Never....................6

Don't know/no answer.........

More than once a week.........1

About once a week............2

One or two times a month.......3

Less than once a month........4

One or two times a year.......5

Never....................6 6

Don't know/no answer......... 
28. Think for a moment about a situation in which you arive within one hour of having had too much to drink. In your own estimation, if you did this 100 times, how many times would you be caught and punished?

(RECORD EXACT RESPONSE)

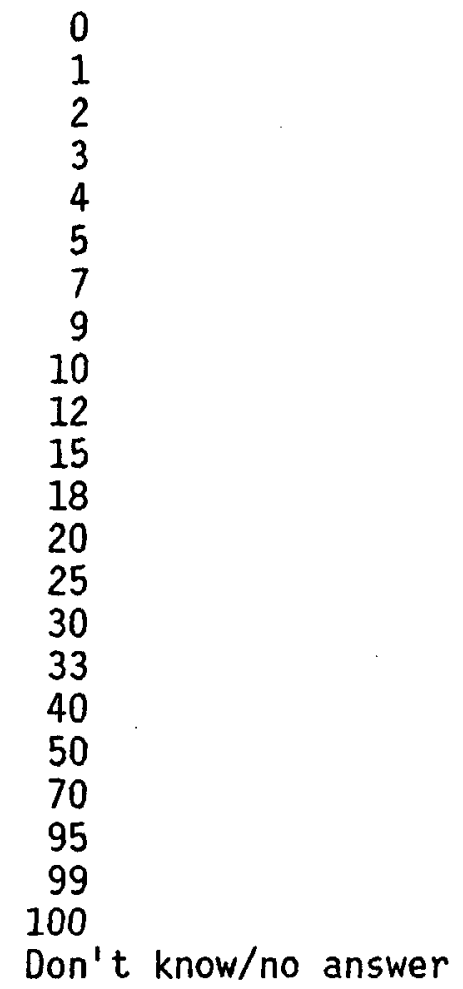


29. How high would the number have to be in order for you to decide not to drive after having had too much to drink?

(RECORD EXACT RESPONSE)

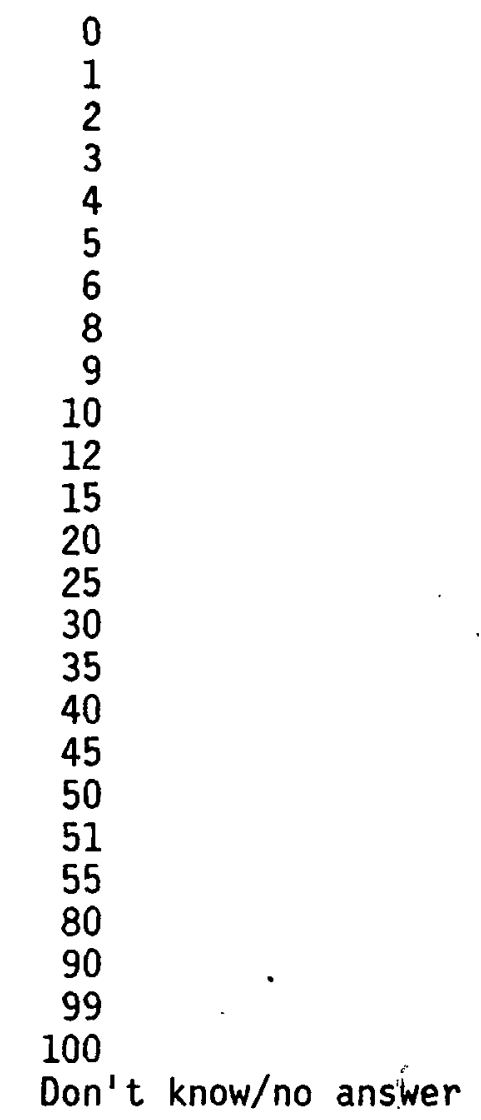


30. Have you personally made any efforts to reduce the number of times you have driven after drinking too much in the past 6 months?

Yes $($ TO 31$) \ldots \ldots \ldots \ldots \ldots \ldots 1$

No $($ TO 33$) \ldots \ldots \ldots \ldots \ldots \ldots . .2$

Don't know..................

IF "YES" IN QUESTION 30, ASK:

What have you done? (DO NOT READ, CHECK EACH THAT APPLIES)

(FOR EACH MENTIONED, ASK: Is that something you have started to do recently? RECORD BELOW IN THIRD AND FOURTH COLUMNS FOR EACH ACTION MENTIONED.)

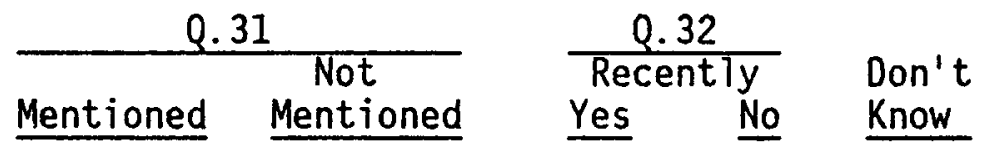
a) Don't drink alcoholic
beverages

b) Limit the number of drinks

c) Eat food

d) Drink some alcoholic beverages and some non-alcoholic beverages

e) Stop drinking at a predetermined time

f) Had someone else drive home

g) Took a taxi/public transportation

h) Test self for own alcohol level

i) Stayed overnight

j) Drank coffee

k) DiTuted drinks

1) Other (SPECIFY)

\begin{tabular}{|c|c|c|c|c|}
\hline $19 \%$ & $81 \%$ & $15 \%$ & $7 \%$ & $79 \%$ \\
\hline $38 \%$ & $62 \%$ & $26 \%$ & $12 \%$ & $62 \%$ \\
\hline $5 \%$ & $95 \%$ & $3 \%$ & $5 \%$ & $93 \%$ \\
\hline $3 \%$ & $97 \%$ & $1 \%$ & $4 \%$ & $95 \%$ \\
\hline $15 \%$ & $85 \%$ & $7 \%$ & $9 \%$ & $84 \%$ \\
\hline $36 \%$ & $64 \%$ & $23 \%$ & $14 \%$ & $63 \%$ \\
\hline $3 \%$ & $97 \%$ & $2 \%$ & $4 \%$ & $95 \%$ \\
\hline $1 \%$ & $99 \%$ & $=$ & $3 \%$ & $97 \%$ \\
\hline $4 \%$ & $96 \%$ & $4 \%$ & $3 \%$ & $94 \%$ \\
\hline $4 \%$ & $96 \%$ & $3 \%$ & $3 \%$ & $94 \%$ \\
\hline $1 \%$ & $99 \%$ & $1 \%$ & $3 \%$ & $97 \%$ \\
\hline $13 \%$ & $87 \%$ & $11 \%$ & $5 \%$ & $84 \%$ \\
\hline
\end{tabular}


33. How can you tell when you have had too much to drink to drive safely? (DO NOT READ, RECORD RESPONSES ONLY -- DO NOT PROBE)

\begin{tabular}{|c|c|c|c|}
\hline & & Mentioned & $\begin{array}{c}\text { Not } \\
\text { Mentioned }\end{array}$ \\
\hline a) & Number of drinks & $12 \%$ & $88 \%$ \\
\hline b) & Slurred speech & $5 \%$ & $96 \%$ \\
\hline c) & Weaving/staggering & $8 \%$ & $92 \%$ \\
\hline d) & $\begin{array}{l}\text { Personality change, such as very } \\
\text { talkative, loud, aggressive, very } \\
\text { quiet, etc. }\end{array}$ & $6 \%$ & $94 \%$ \\
\hline e) & Can't stand up & $3 \%$ & $97 \%$ \\
\hline f) & Sleepy & $5 \%$ & $95 \%$ \\
\hline g) & General behavior & $8 \%$ & $92 \%$ \\
\hline h) & Passed out & $1 \%$ & $99 \%$ \\
\hline i) & Can just tell (nothing specific) & $8 \%$ & $92 \%$ \\
\hline j) & Can't tell & $6 \%$ & $94 \%$ \\
\hline k) & Other (SPECIFY) & $19 \%$ & $81 \%$ \\
\hline
\end{tabular}

I am going to read you a statement. Please indicate whether you agree or disagree --

34. "Except for alcoholics and persons with real drinking problems, few people drive after drinking too much to drive safely."

$\frac{\text { IF CHOICE MADE, ASK: }}{\text { you feel strongly about that? }}$

Agree/strongly.............

Agree......................

Unsure (DO NOT READ) ...........

Disagree....................

Disagree/strongly............ 5

35. About how many miles did you personally drive during the past 12 months?

$0-1,000 \ldots \ldots \ldots \ldots \ldots \ldots \ldots \ldots 1$

$1,200-5,000 \ldots \ldots \ldots \ldots \ldots \ldots \ldots .2$

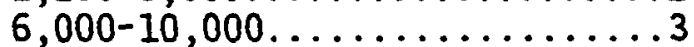

$11,000-15,000 \ldots \ldots \ldots \ldots \ldots \ldots . . \ldots 4$

16,000 and over............. 5

Don't know/no answer.......... 6 
36. About how many miles do you drive in an average week day (Monday-Friday)?

$0-10 \ldots \ldots \ldots \ldots \ldots \ldots \ldots \ldots$

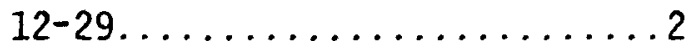

$(27 \%$

$30-69 \ldots \ldots \ldots \ldots \ldots \ldots \ldots \ldots$

$(19 \%$

70 and over..............

Don't know/no answer.........5

37. About how many miles do you drive on an average weekend (Saturday-Sunday)?

$0-10 \ldots \ldots \ldots \ldots \ldots \ldots \ldots \ldots$

$11-20 \ldots \ldots \ldots \ldots \ldots \ldots \ldots \ldots 2$

$21-50 \ldots \ldots \ldots \ldots \ldots \ldots \ldots \ldots \ldots$

Over $50 \ldots \ldots \ldots \ldots \ldots \ldots \ldots \ldots$

$(27 \%$

Don't know/no answer.........5

38. How many automobile trips have you

taken in the last year that were over

200 total miles?

$0-1 \ldots \ldots \ldots \ldots \ldots \ldots \ldots \ldots \ldots$

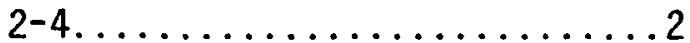

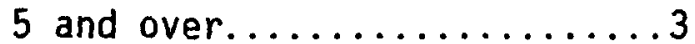

Don't know/no answer.........4

39. Is this more or less than the year before?

More...................

Unsure (DO NOT READ). . ....... 2

No difference (DO NOT READ) .... 3

Less.....................4

40. In what month and year was the last trip you took which was over 200 total miles?

Year of Last 200. Mile Trip

None

Before 1980

1980

1981

1982

1983

Don't know/no answer

$\begin{array}{r}3 \% \\ \hline 8 \% \\ \hline 3 \% \\ 7 \% \\ \hline 47 \% \\ 28 \% \\ 4 \% \\ \hline\end{array}$

Season of Last 200 Mile Trip

None

Fall/Winter

Spring/Summer

Don't know/no answer
$3 \%$

$54 \%$

$36 \%$

41. How many people were in the car on

that trip?

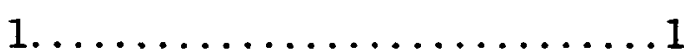

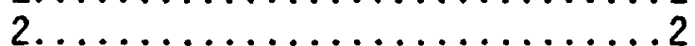

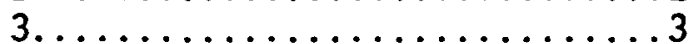

4 or more...............4

Don't know/no answer..........5 
42. Do you think you and your family drove more in 1982 than you did in 1981, or less in 1982 than you did in 1981?

More (T0 43-1)..............

Less (TO $43-2) \ldots \ldots \ldots \ldots \ldots \ldots 2$

The same (TO 44)............

IF "MORE" IN QUESTION 42, ASK:

43-1 And why did your family drive more in 1982?

01. VACATION - because of our whole family going on vacation/ we took a vacation/we went on our vacation/we just drove on vacation instead of flying/took vacation to another state/

02. MORE/LONG TRIPS - took more trips/we went on a lot of trips/ took long trips in 1982/we took a $6000 \mathrm{mile}$ trip/more frequent short trips/

03. NEW JOB LOCATION - change in job, I used to live where I worked/we went more to Atlantic City because of new job/ I'm commuting for a job now/my office moved further away/ new location for my job/

04. BUSINESS TRIPS - mostly business trips/I took the family with me more on business trips/my business required it/ working out of town now/more business-related trips/

05. VISITING FAMILY/FRIENDS - our son moved to Kansas and we go to see him quite a lot/more relatives that live further away/my children have moved to different states and I go to visit them/we visited more friends/

06. ILLNESS/DEATH - we had to drive 120 miles to see my mother-

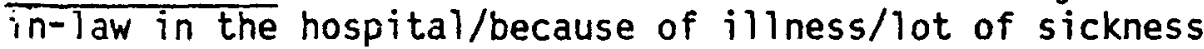
in immediate family/had to make several trips to the hospital/had death in family, needed to travel/

07. MORE PLACES TO GO - we had more places to go/went more places last year/we went different places/going to more places now than in past years/

08. MORE TIME - had more time off/we had more time to travel/ more time off from the job/my husband retired and we had more time to go places/have more time to trave1/

09. LOWER GAS PRICES - the Towering of gas prices in 1982/gas was little bit cheaper last year/the price of gasoline went down/ 
Question 43-1 continued

10. MORE DRIVERS IN FAMILY' - we had more drivers in the family/ we have a third driver, our son got his license/more people in the family drive now/

11. MORE MONEY - we made more money/we could afford it/had more money to travel/financially better off/I went back to work and we had more money/

12. BOUGHT SECOND CAR - we acquired another car/we got another car/we bought an additional car/we bought a second car/

13. COLLEGE - taking kids back and forth to college/my daughter. went off to college/more trips to college/I went to school 30 miles away every day that year/

14. TRAVELED MORE - we were traveling more/we spent the last summer traveling quite a bit/went away more/we decided to travel all over the world/

15. CHILDREN OLDER/MORE ACTIVITIES - kids are older and they go more places/kids are older and have a greater demand for transportation/we were involved in more youth activities/

16. MORE TO DO - more things to do/more family gatherings/doing more things/family things coming up/

17. MORE FAMILY MEMBERS - my family was larger then/more working members in the family/more of us/there were more people in the family/more family members/

18. MORE OPPORTUNITIES - more opportunity to trave1/more opportunities and invitations to do more things/we had more opportunity to go/we just had more social engagements than the year before/

19. CAMPER/MOTOR HOME - we have a camper now/we bought a motor home and have taken more vacations/we bought a motor home ...we are seeing the U.S.I

20. WIFE/CHILDREN WORKING - my wife took a job and had to drive about 70 miles per day/because my kids have jobs/

21. MORE ERRANDS/VOLUNTEER WORK - I have more errands to run than I used to/more running around/more volunteer work/

22. GOT LICENSE - I got my license/my brother and I got our licenses/I just received my license/

23. CAR POOL - my children go to Catholic school and I have to drive them/I drive a car pool, drive children to school 10 miles away/ 
Question 43-1 continued

24. HAD OWN CAR - we had our own car and didn't have to borrow/ I owned my car that time so I drove a lot then/

25. JUST HAPPENED THAT WAY - it just happened that way/it was just the way things happened/just coincidence/

26. QUT OF COUNTRY IN 1981 - in 1981 we lived in Korea and weren't many places to drive/we were in England in ' 81 /

27. OTHER

28. DON'T KNOW/NO ANSWER

IF "LESS" IN QUESTION 42, ASK:

43-2 And why did your family drive less in 1982?

01. GAS PRICES/AUTO EXPENSES - cost of gas/the gas price going up/high-priced gas/I'm cutting back on use of the car because of the high cost of gas and maintenance of the carl cost of operating a vehicle/we can't afford the gas/

02. TOOK FEWER/NO TRIPS - we took less long distance trips/ took less family trips/we haven't taken the trips that we had previousiy/didn't take any trips/we went several places the year before/we took a long trip in ' 81 and didn't in '82/

03. ECONOMY - the high prices of everything/with the economy like it is, we just couldn't afford to/cost, expensive for food, gas and lodging/the money was tighter/the economy/

04. ELDERLY/RETIRED - we're getting older and don't have the energy/I'm 70 years old, so I don't drive as much as I used to/I'm retired and I can't see too well/we' re both retired now and don't have to drive to work/

05. NO/LESS MONEY - no dough, no go/there was no money for gasoline/income was lower/ain't got no money to go nowhere/we had a lot less money in 1982/no money/

06. NO VACATION/LESS VACATION TIME - didn't take a vacation/ couldn $t$ afford a vacation/we had less vacation time last year/a combination vacation was planned closer to home/ I didn't have as much vacation time/

07. DIDN'T GO ANYWHERE/NOWHERE,TO GO - we just didn't have any places to go/we had no reason to go any place/just don't go much anymore/just don't go:anywhere/just sit home a lot/ 
08. PREFER NOT TO/PERSONAL LIFE STYLE - lack interest, didn't go anywhere/didn' $t$ feel like going anywhere/life style/ cut down on driving, I'm tired of driving/I didn't choose to drive as much/we got in a rut and didn't take of $f$ on weekends as much/

09. FAMILY ILLNESS - family $i 11$ ness/my husband was in the hospital/I was taking care of my mother and she was very sick/my husband was injured at work/my wife is not well/ I was taking care of a sick aunt/

10. PERSONAL HEALTH - open-heart surgery and didn't go around too much/I was in the hospital a couple of months/my health, my wife can't drive and I was $i 11$ /eye problems/

11. NO TIME/BUSY WORKING - we both had jobs and were working and didn't have time to take a trip/I was too busy to go anywhere/lack of time/no free time/

12. ALTERED WORKING CONDITIONS - because of changing working conditions/husband worked different hours/it was harder for me to get out of work/my 'girl friend don't have weekends off anymore/schedule of my job/

13. LESS JOB-RELATED DRIVING - not as much business trips as 1981/I was a salesperson in 1981/I used to be a driver at my job... no longer am at that job/my job doesn't require as much this year/

14. USE TRAIN/PLANE - we have more money and fly more/our vacation was different, we took a plane in ' $82 /$ I go on the train a lot now/we fly most of the time/we take a train or plane/

15. CLOSER TO WORK/TOWN NOW - I work in town now/moved closer to our job/I live closer to work/we live closer to town/

16. UNEMPLOYED - we were unemployed in the year 1981/I quit work so I don't leave the house as much/I was laid off from work/husband was unemployed/

17. CAR TROUBLE/NO CAR - we had trouble with the car/I don't have a car so I only drive a rental car or a borrowed car/ our car gets bad mileage/my car was getting old/the family owned less vehicles in 1982/

18. FAMILY CLOSER NOW - my daughter lives closer now/we all live closer together/the family moved closer to me/I moved closer to my family/

19. DIDN'T VISIT RELATIVES - I didn't go and visit my relatives as much/I didn't go visit my son who lives about 400 miles from here round trip/we did not have the opportunity to visit family as much/ 
Question 43-2 continued

20. PERSONAL CIRCUMSTANCES - personal reasons/I don't drive anymore/it was a complicated year, we had a lot of personal problems/I was overseas in 1982/we got a pool so we don't go anymore/

21. DON'T GO OUT AS MUCH - just the two of us and we don't go out much anymore/we were doing something every weekend in 1981 and in 1982 we quit/didn't go out as much last year/ we don't go as much because it is hazardous to drive around in the traffic/we went to more concerts the year before/

22. SCHOOL-RELATED MENTIONS - my daughter started college/I am working for a master degree and am really saving/my wife is in school/my son has graduated from college so we have stopped making the trips to see him/

23. FAMILY CHANGES - I got divorced/in 1981 I was pregnant/my wife was pregnant at the time/new baby, we were not able to more around much/my father passed away/I lost my wife in ' 82 and I don't feel like going places anymore/

24. RIDE SHARING/FUEL CONSERVATION - I car pool now/rearranged work schedule where my wife and I ride together/I share my driving with other people/my son-in-law drives us on trips/. we're conserving on gas/

25. NO REASON

26. OTHER

27. DON'T KNOW/NO ANSWER

44. In general, have your oriving habits -trip length, where you drive, when you drive, how you drive -- changed in the last year?

Yes (TO $45 \& 46) \ldots \ldots \ldots \ldots . . . .1$ Unsure (DO NOT READ) (TO 47) ...2

No $($ TO 47$) \ldots \ldots \ldots \ldots \ldots \ldots$

IF "YES" IN QUESTION 44, ASK:

45. In what way have your driving habits changed?

01. MORE CAUTIOUS - I've become more cautious/I am a lot more careful/I'm more observant and cautious than I used to be/ more precaution toward other drivers now/I'm more cautious of people around us/

02. DRIVE SLOWER - I have slowed down more/slowed way down/ drive slowly, more content with the speed limit/I don't drive as fast as I' used to/ 
Question 45 continued

03. DRIVE LESS - I drive less/I'm not driving as much as I was/don't drive as much because don't work much/I've joined a car pool so don't drive to work everyday now/

04. DEFENSIVE DRIVING - driving more defensively/more defensive driver/we moved into a new area and have to drive more defensively/try to drive more defensively/

05. DRIVE MORE - I'm driving more/I drive a lot more/I am driving more highway miles than a year ago/

06. USE SEAT BELT - I use the seat belt always now/started wearing seat belts/I use seat belts more/

07. AVOID DRINKING - less frequency of drinking/I don't drink at al1 anymore/stop drinking and driving/avoid driving when I drink/don't drink anymore/

08. MORE AWARE/ALERT - I'm more aware of what's going on around us/I'm more aware of other people than I used to be/I'm more aware of the way I drive/we have to be much more alert/I trained myself to be more alert/

09. OBSERVE SPEED LIMIT - obeying speed $1 \mathrm{imit} / \mathrm{I}^{\prime} \mathrm{m}$ more abiding with the speed Taw/drive speed limit/

10. CHILDREN IN CAR - addition of child makes me more cautious/ I travel with children now/I've become more concerned with the safety of my children/cautious because of little children in the carl

11. AWARE OF OTHER DRIVER - I watch other people a whole lot more/I watch the other person more/more cautious of other people's driving/really watch out for people who drive crazy/

12. SAFETY CONSCIOUS - improved safety habits/I'm more conscious of safety/took a defensive course class and now am more aware of my driving/

13. OLDER/MORE CAREFUL - getting oider makes you more careful/ when you get older you drive a little different/

14. AFFECTED BY WEATHER CONDITIONS - I refuse to drive on ice anymore/I'm more careful in weather conditions now/dictated by the weather/if the weather is bad, I don't drive/

15. SHORTER TRIPS - shorten my trips, get more things when I go to town/do more errands in one trip/I try to take as ittle number of trips as possible/ 
Question 45 continued

17. LONGER/MORE TRIPS - only use it for long trips/longer trips/ my husband retired and we take more trips in the country/

18. MORE DAYTIME ORIVING - more daytime driving/I changed from driving at night to day/we try not to drive at night/

19. CHANGED RESIDENCE/JOB - we moved to the country/we just moved to a better town/I moved to a different community/ I've changed jobs/

20. BETTER/NEW CAR - I got a better car/I bought a new car/

21. NERVOUS/TENSE - I am more nervous to get on the highway/I notice when you get tense it affects your driving/

22. OTHER

46. And why did you change your driving habits?

01. WORK RELATED - change in occupation/my new job is closer for me/my employment is further away now/more business so we need to drive more/

02. SCHOOL RELATED - I started back to school/I go to a closer school now/because I'm no longer in school/

03. OUT OF WORK - I got laid off/don't have a job/

04. CHILOREN - I have had children/young children to protect/ my children are very important to me/our children/

05. MATURITY/WISDOM - just wised up/that goes with age/I became more settled and got older/it comes with age/you just get older and wiser/

06. OLD AGE/RETIRED - just getting to old/because we have retired/because we're old/as I get older, my responses diminish/

07. COST/ECONOMY - no money/cost factors/more car pooling/for economic reasons/the economy/bad economy condition/

08. ACCIDENT/SELF - I had an accident/I had a wreck on my motorcycle/wrecked two Corvettes and one other car/I have realized after being in an accident just how many turkeys are out there/I had an accident and learned from it/ 
Question 46 continued

09. ACCIDENT/FAMILY/FRIENDS - my friend was in a car accident and was injured badly/an accident in the family/my father was killed in an auto accident/

10. AWARE/AVOID ACCIDENTS - seen too many accidents/more aware of the accidents/so many accidents you hear about/trying to avoid accidents/had a few close calls lately/

11. OHER DRIVERS - more drunk drivers on road/the people don't seem to look where they're going/to protect myself from those aggressive drivers/more bad drivers on the road now/ drive for yourself and other person too/

12. GOT CAUGHT - I got a speeding ticket/I got so many tickets and lost my license two times/my last DWI got my attention/ I was very embarrassed about being arrested/

13. LAW CHANGES - speed limit change/the new laws/they raised the ticket prices/tougher laws on drunk drivers/

14. SAFETY/LESS DANGEROUS - for my own safety/I feel safer/ personal safety and safety of others on the road/it's safer/danger involved/

15. INCREASED PUBLICITY/AWARENESS - all the programs about drunk driving/knowledge through publicity/more awareness of possible outcomes/I'm more aware, the whole public is more aware/

16. MOVED - I changed residence/we moved/I live in Manhattan now and sold my car'

17. TRAFFIC - more traffic/too many cars on the highway/don't Tike the traffic congestion/

18. DIFFERENT CAR - I have a car I can depend on/got a new car/changed cars/I got a 502/

19. SPECIFIC PRESENTATION - defensive driving class/because of that telephone cal1 a year ago about seat belts/in that class I saw the bad points of my driving/saw a film on seat belt use/

20. TO STAY ALIVE - just to stay alive/I want to live/was putting my life in jeopardy/

21. DRIVE LESS - just not traveling as much/we stay at home/ flew more in the last year/

22. DRIVE MORE/MORE TRIPS - more long distance trips/I drive morel 
Question 46 continued

23. DON'T ORIVE MUCH - I hate driving/never did drive that much/ don't drive anymore/don't need to drive/easier to ride the bus/

24. PERSONAL/FAMILY REASONS - I have care of my mother who is il1/I have been sick/my children are in school and I have more time/I began staying home and raising a family rather than working/I was saved/

25. OTHER

26. DON'T KNOW/NO ANSWER

47. Now think back to the period between

Thanksgiving and New Year's -- last

year, that is, 1982. Did you use your

car more than during the previous holiday

season, or less than during the previous

hol iday season?

More $($ TO $48-1) \ldots \ldots \ldots \ldots \ldots 1$

Less $($ TO $48-2) \ldots \ldots \ldots \ldots \ldots . \ldots 2$

The same (TO 49)............

Unsure (DO NOT READ) (TO 49) ...4

IF "MORE" IN QUESTION 47 , ASK:

48-1 And why did your family use your car more this holiday season than the previous one?

01. VISITING RELATIVES - we went to visit my sister more/my daughter lives out of state/we went to visit family/we visited relatives more/our family lives farther away/

02. TOOK A TRIP - because we took a trip to Indiana/we took a trip to Georgia during the holidays/we traveled East for Christmas/went out of town/

03. VISITING/VISITING FRIENDS - we did more visiting this holiday season/we went visiting with holiday guests/more people to see/we were visiting friends/some of our friends moved close enough to drive to/

04. MORE PLACES TO GO - more places to go/there was more to do this year/we had more parties to attend/more to do/because we went out more to different places/

05. JOB RELATED - I got a part-time job/I had to work/I have a new job and drive to Jersey City every weekend/I was further from my job last season/ 
Question $48-1$ continued

06. SHOPPING - more shopping trips/went shopping more/I had more shopping to do this holiday season/

07. SCHOOL RELATED - I was in school and was driving back and forth/because I go to school/more college trips/

08. MEDICF.L RELATED - because I went to the doctor more/because my. wife was hospitalized and I was going back and forth to see her/

09. BETTER WEATHER - the weather was nicer so we went on more trips/the weather was better/

10. MORE TIME/MONEY - more free time/we weren't as busy so we had time to go to more social outings/I was not working and I had more time for useless trips/my husband was off on weekends/I had more money/

11. OUT-OF-TOWN GUESTS - we had out-of-town company and went out more/because we had out-of-town guests and we took them out showing them around/my sister was visiting and went to take her sightseeing/

12. MORE/BETTER CARS - we had an additional car to use/better carlour car was working this season/we only had one car and now we have two, so we're both driving/

13. MOVED - we were in the process of moving to Idaho/I moved/ we moved during the holiday season/

14. MORE DRIVERS - more people could drive in the family/more people driving/I had my. license/

15. MARITAL STATUS - because I was single/change of marital

16. OTHER

17. DON'T KNOW/NO ANSWER 
IF "LESS" IN QUESTION 47, ASK:

48-2 And why did your family use your car less this holiday season than the previous one?

01. STAYED HOME - we didn't go any place/we stayed home this year/just didn't/I just don't go anywhere/family stayed home more this year/we just celebrate at home/

02. OTHERS CAME HERE - company came over/everybody came to our house for the holidays/my mother came here instead of our going there/it was my turn to have everyone/rotating family Christmas/

03. TOO EXPENSIVE - we were broke/cutting back to save money/ money shortage/too expensive/the poor economic situation/ less money to go places/

04. STAYED LOCAL - we didn't go away on a trip/I stayed in the local area/didn't go out of town/we just went a block/we just stayed with neighbors/

05. FAMILY ILLNESS/ACCIDENTS - I was in traction for 6 months/ we were both sick/we had some colds/I broke my leg/Mom was i11/

06. FGMILY CLOSER NOW - family a couple of miles away from home/ our family was pretty close by/the family was a lot closer than before/most of our family has moved closer to us/

07. LESS SOCIALIZING - we didn't go out as much/wasn't no one at home/I don't have as much demand socially/attend less social events/I didn't have anywhere to go/

08. WORKED/LESS TIME - wife worked/my job changed and I put in more hours/had to work on the holidays/I have a business and cannot leave it/I didn't have time this year/

09. CAR PROBLEMS/NO CAR - I did not have my car the previous year/the way my car uses up gas/I had an accident and we were without a vehicle for a short while/car trouble/

10. TRAFFIC FEARS - too much holiday traffic/accidents increase during the holiday season/so many people on the road who have been drinking/traffic in Florida was bad/

11. WEATHER CONDITIONS - we couldn't get out of town because of bad weather/I don't like to drive through the snow/ weather conditions/there was a blizzard/

12. GAS PRICES/PROBLEMS - because of the gas problem/the higher gas prices/gas prices too high/cost of gas/

13. TOOK A PLANE/TRAIN/BUS - we flew instead of drove to be with our family/I went on a plane/we rode the bus/went on a train/ 
Question 48-2 continued

14. OIDN'T VISIT FAMILY/TOO FAR - we didn't go to see my family/didn't go visiting any relatives/didn't make the usual visit to my parents/didn't go to my brother's house/away from my family and relatives/daughters are farther away/

15. UNEMPLOYED - lack of employment/I was unemployed this year/ my husband was laid off/I quit work/

16. ELDERLY/RETIRED - too old to go anymore/we're retired/it's due to my age/

17. CHANGED SHOPPING HABITS - I didn't take long shopping trips/ I shopped earlier/didn't drive around Christmas shopping as much/we have been shopping by mail/I don't shop with my wife anymore/do less shopping/

18. OTHERS DROVE - someone else drove/traveled with son and his family/a friend driving instead of myself/traveled with other people/

19. NOT HOME - we were out of state/stayed in New York all through the holidays/we were in California/I was overseas and didn't drive much/

20. DEATH IN FAMILY - my mother died/death in family/I was by myself due to my husband passing away/

21. ALTERED LIFE STYLE - married this year/I was pregnant/the baby was just born a week before Christmas/we moved/we relocated from the North to the South/

22. OTHER

23. DON'T KNOW/NO ANSWER

49. Have you ever heard of Mothers Against Drunk Drivers, or MADD?

Yes (TO 50-1 \& 50-2) .......... Unsure (DO NOT READ) (TO 52)...2

No $($ TO 52$) \ldots \ldots \ldots \ldots \ldots \ldots$ 
IF "YES" IN QUESTION 49, ASK:

50-1 And can you tell me what MADD is or what they are trying to do?

01. PREVENT DRUNKEN DRIVING - trying to keep hard-core drinkers off the road/trying to get drunk drivers off the road P.T.A. is for them/prevent drunk drivers to keep them off the street/trying to stop drunk drivers from getting back on the road/trying to get drunks off the highway/

02. WANT STRONG LAWS - organization trying to strengthen drunk driving laws/to get legislation passed to get drunk drivers off the road/to get better laws against drunk driving/ their objective is to get more laws passed to be harder on drunk drivers/a group of mothers getting together trying to get stiffer legislation against drunk drivers/

03. WANT SEVERE PENALTIES - they are trying to get stricter penalties against drivers convicted of manslaughter due to drunk driving/they are trying to get stiffer fines and jail sentences for drunk drivers/the people involved are getting more severe punishment for drunk drivers/ they need stronger punishments/

04. PERSONALLY AFFECTED/DRUNK DRIVERS - an organization started by a woman whose son was killed by a drunk driver/they all have had a child killed in a car accident/a group started because children were killed by drunk drivers/parents of maimed children/mothers who have had their children killed by drunk drivers, and they try to console other victims/

05. REDUCE DRINKING-RELATED DEATHS/ACCIDENTS - prevent people driving when drunk and people getting killed/trying to keep drunk drivers off the road so that they will not kill the children/trying to decrease the number of highway deaths/trying to cut down on drunk driving deaths/ lower number of accidents/

06. STRICTER ENFORCEMENT - trying to enforce strict laws against D.W.I./stricter enforcement of drunk driving laws/trying to convict drunk drivers/

07. STOP DRINKERS FROM DRIVING - trying to keep people who drive off the roads/trying to put a stop to folks driving and drinking/they formed a group to keep people from drinking and driving/attempting to get the drinkers out from behind the wheel of a carl

08. AGAINST DRUNK DRIVERS - their efforts are against drunk drivers/control drunk drivers in this nation/getting the state to crack down on drunk drivers/women striking back at drunk drivers/against drunk drivers/complaining about drunk driving/angry at drunk drivers/ 
Question $50-1$ continued

09. EDUCATE PUBLIC - to make people more aware of drunk drivers/ trying to develop a groundswell against drunk driving/ they are trying to bring the public some education/make drunk drivers more aware of the punishments they will receive/educate young people not to drink and drive/

10. JAIL SENTENCES FOR D.W.I. - they are trying to get mandatory jail sentences for drunk drivers/put drunk drivers in jail for 48 hours and if they have had an accident and killed... put them in prison/

11. SAW MOVIE/PUBLICITY - I saw a movie about them a few weeks ago/all I know is what I saw on T.V. about them/ seen a headline in the newspaper about that/I've just heard of them/

12. RAISE DRINKING AGE/STOP TEEN DRUNKS - enforcing laws for teens not to drive during or after drinking/keep kids under 25 from drinking/get those kids off the road/ they are trying to have the drinking age raised/

13. OTHER

14. DON'T KNOW/NO ANSWER

IF "YES" IN QUESTION 49, ASK:

50-2 Do you think Mothers Against Drunk Drivers is succeeding?

Yes $($ TO 51)................. Unsure (DO NOT READ) (TO 52)....2 No $($ TO 52$) \ldots \ldots \ldots \ldots \ldots . \ldots 3$

51. And in your own words, what are one or two reasons why you feel that way?

01. NEW/STRICTER LAWS - passed new drunk driving Taws/laws in Colorado have gotten stricter partly due to this organization/just passed tougher laws here/we've had tougher drunk driving laws passed in the past year/

02. PUBLICITY - they get lots of publicity here/in advertising/ from hearing a lot about them on the news/hear more about it/they've gotten more publicity/they are getting more publicity than before/

03. PUBLIC AWARENESS - bringing awareness to the problem/ people are paying more attention to the problem/people are becoming more aware of the dangers of drunk drivers/ more Deodle are aware of the problem/ 
Question 51 continued

04. STIFFER/INCREASED PENALTIES - in New York state there's a

real push to get stiffer penalties/forcing the states to pass stiffer legislation against drunk drivers/drunk drivers are getting tougher sentences and more fines/ you get jailed for the first drunk driving offense now in Pennsylvania/

05. TELEVISION PRESENTATION - I happened to see their televised drama last week/I watched it on TV and I think they have expanded/I saw the movie last week/through the media/more known, especially on the media/

06. ENFORCING D.W.I. LAWS - police are really enforcing drunk driving Taws/more arrests and convictions are being made/ in Seattle they are really cracking down on anyone caught drinking and driving/

07. WHAT I'VE SEEN/READ/HEARD - I'm hearing on news and in the paper/more publicity in newspapers/they're introducing their program to the media/from what I've read they've been successful/

08. RECOGNITION OF ORGANIZATION - recognition/they are succeeding because they are getting a lot of attention/getting more recognition now/

09. PERSONAL EXPERIENCES - because of my own experience/she had a friend lose her life because of drinking/there's been drunk drivers in my neighborhood/we were hit by a drunk/ we had a few kids killed by drunk drivers in this area/

10. GROWING/EFFECTIVE - more pub1ic involvement/they are doing what they set out to do/more and more people becoming involved in this issue/they put on a good campaign/they've got more people joining now/

11. FEWER DRUNKEN DRIVERS/ACCIDENTS - number of accidents due to alcohol has dropped/looking at the obituaries you can te 11 it's much less/less people on the road that are drunk/less accidents because of drunk drivers/getting many off the streets/

12. DOING A GOOD JOB - they are getting the message across/ it's very valuable, accomplish a lot/strong lobby and determination of them/people are paying more attention to the problems/it is a good cause that people are willing to support/

13. DON'T LIKE DRUNK DRIVERS - they've got to crack down on drunk drivers/don't think drunk drivers should be on the road/too many drinking drivers/drunk drivers hurt innocent bystanders than they hurt themselves/ 
Question 51 continued

14. NEED STRONGER PUNISHMENT - it's better but the punishment isn't hard enough sti17people are still getting away with drunk driving and killing people without a harsh punishment/some just get away scot-free/

15. NEED EDUCATION - "any" education in the public the better the chances are of getting results to the problem/goou way to run them off the road '- by' educating others of the problem/educating lot of people/a lot of people who drink and drive are educated and through education they realize not to drive and drink/

16. PARENTAL REACTION - concern for their kids/lots of mothers and fathers who are complaining about drunk drivers now/ I've got children and would hate to see them get hit by a drunk driver/the mothers are upset about their kids and relatives being injured and killed/

17. NEED MORE TIME/SCOPE - it has helped but they need more time/they are not reaching enough to many judges and police/hasn't been a major change so far but their future looks hopeful/still have a long way to go/

18. NEEDLESS DEATHS/ACCIDENTS - it will stop deaths/seeing wrecks with young children involved/if they take someone's life they should be punished for it/they're just killing a lot of innocent people/

19. PROBLEM DRINKING - alcoholism is a problem/our society drinks too much/I don't believe in drinking/

20. TRY HARDER/GET INVOLVED - feel that people should try harder/if they can do something about drunk drivers they should at least try/not enough people involved/

21. TAKING A STAND - people are speaking up for what's going now/I hear peer groups are concerned about drunken driving by talking about it/

22. LEGISLATIVE ALLIES - they are complaining to the law about the drunk drivers/we have some bills in town and legislature - they will probably get passed/

23. BENEFITS ALL - it might make driving safer/it benefits everyone/we17 safety of all of us/

24. MAKING AN IMPRESSION - it seems like it's hitting home/ children seem to be getting the message/you can tell the difference between the ones who are driving now than one year ago/ 
Question 51 continued

25. SERIOUS MATTER - it's such a serious problem/it's a very serious thing and people listen to it/don't see how you can live with it if you killed a child/

26. OTHER

27. DON'T KNOW/NO ANSWER

52. Which of the following best describes your use of safety belts compared to a year ago? (READ LIST)

1) I use them significantiy more than I used to. $9 \%$

2) I use them slightly more than I used to. $10 \%$

3) I use them about the same. $72 \%$

4) I use them slightly less than I used to. $3 \%$

5) I use them significantly less than I used to. $4 \%$

6) Don't know/no answer $2 \%$

IF "SIGNIFICANTLY MORE" OR "SLIGHTLY MORE" IN QUESTION 52, ASK:

53. And what are one or two reasons why you are using safety belts more than you used to?

01. TO BE SAFER - I believe they are safer/safety reasons, they work/because they save lives/to cut down on injuries/to protect myself more/I'm becoming brainwashed that they might be a little safer/it makes me feel more safe/trying to be more careful/they save lives/

02. FOR CHILDREN - have more children in the car since I drive them all to school/concern for my children/my children use them now/mostly for my son, to protect $\mathrm{him} / \mathrm{had}$ a son, it really makes you more cautious in everything you do/I'm starting a family/children need to be in a safety harness always/

03. MORE CRAZY/DRUNK DRIVERS - I've noticed crazier people out there on the road/more people are speeding and drinking/ because of all the drunk drivers/don't trust other drivers/ there are more bad drivers on the road/ 
04. PERSONAL EXPERIENCE/WRECKS - I had to stop quickly and my five year old lost several of her front teeth/I was in an accident and my seat belt saved me/I didn't have them in my truck when I wrecked it - I chipped my front teeth on the steering wheel/got a knee injury I don't think I would have if I had had on my seat belt/my wife had a serious accident/

05. G000/NEWER CAR DESIGN - I have a buzzer in my car which reminds me to fasten my seat belts/I have a new car and they are built right into it/my car will not start without the seat belts fastened/I have a new car and it's easier and more convenient/we got a new car with good seat belts/

06. PUBLICITY/FILMS - publicity/the advertisements/articles in paper/I saw on TV if you use seat belts it's safer/ seeing safety films at work/the campaigns going on here ads on TV/we are deluged with it/

07. MORE AWARE - more of an awareness of the importance of using them/I'm just more aware of the risks than I was a year ago/I think about it more/a little more awareness of the chance of being in an accident/I am more aware of the harm it does the older I get/

08. TRAFFIC PROBLEMS/ROAD CONDITIONS - because of the problem of traffic congestion/there are curves and hills, more hazardous conditions/the traffic problems are more severe/ the roads are in much worse condition than they ever have been/more people on the roads driving/there are more and more accidents happening everyday/

09. WITNESSED ACCIDENTS/SEEN VICTIMS - I've seen a lot of accidents where people are getting thrown into the windshield/ I have witnessed a few accidents that people were hurt in/. I work in an ambulance and I've seen what happened/I see accidents all the time and they scare me/

10. FORMING HABIT - I'm trying to train myself to use it/getting used to them/trying to get into the habit of using safety belts/it's more of a habit with me now/

11. OTHERS WANT ME TO - my kids make sure I do/good advice from a friend/my husband has encouraged me to/the person I ride with always is encouraging me to/

12. MORE DRIVING/LONGER TRIPS - I do more driving/because of the long distance trips I have to take to school/we have been taking more long trips/I'm driving more and taking longer trips/ 
Question 53 continued

13. NEW CAR/LESS SAFE CAR - I have a small car and I don't feel as safe in it/I'm now driving faster cars/when I got my new car I said I would use them and I do/the foreign cars don't have enough metal around them/a Jeep and it is known to be easily flipped/

14. ATTITUDE CHANGE - getting older, more sense/not in a rush getting into my vehicle/I'm a little more mature/more concerned about my life/I've just calmed down/

15. SET EXAMPLE/TEACH CHILOREN - I'm trying to set a good example for my children/so children will use them/to set an example for the kids/my son took drivers ed course and I'm helping teach him how to drive/I have two little ones to set an example for/

16. CHILOREN'S CAR SEATS - because of the grandchildren - even if I just go a block, I have one question - will I get a ticket if I don't have my grandchildren in. a car seat/I put my baby into a car seat now and so I just go ahead and put myself in a seat belt too/

17. OTHER

IF "SLIGHTLY LESS" OR "SIGNIFICANTLY LESS" IN QUESTION 52, ASK:

54. And what are one or two reasons why you are using safety belts

less than you used to?

01. UNCOMFORTABLE - I am a short person and the seat belt comes across my neck and it's very uncomfortable/they are very uncomfortable/I think we should be able to fit the seat belts to everyone's different sized bodies/I don't think I can move as well in them/pain in the neck, uncomfortable/

02. JUST HABIT/BROKE HABIT - I broke that habit and I never remember to wear them I fell out of the habit and I just never remember/just got into the habit of not buckling up/

03. QUIT TAKING LONG TRIPS - I only take short trips/take fewer trips/it's just a short distance to work/I don't go as far anymore/I take shorter trips/

04. NOT SAFE - my life was saved when I didn't wear one/they couldn't get the lady out of the car, they had to saw it apart/I have a friend who died in an automobile accident because he couldn't get his seat belts off/my last auto accident, I had my belt on then and had a hell of a time getting it off/

05. JUST LAZY - I don't bother to/just lazy/ 
Question 54 continued

06. TO0 MUCH TROUBLE - nuisance, inconvenient/it's a hassle to hook up/they are too much trcuble to dig out from behind the seat/

07. BROKEN BELTS/NO BELTS - have an old car and it doesn't have seat belts/my safety belts are broken/lost in the seats/the belts in my car are old and don't work right anymore/

08. IN A HURRY/DON'T TAKE TIME - always in a hurry, don't take time/I never take the time to think about $i t / I^{\prime} m$ always in a hurry to get in and out of my car/

09. DON'T LIKE TYPE/PREFER OTHER TYPE - I would be strongly in favor of a harness instead of seat belts/I don't like the shoulder harness/

10. NOT EFFECTIVE/NO NEED - I don't believe they help you any/ I didn't feel I needed them/

11. DRIVE LESS - don't drive as much as I used to/driving less since I fell and I'm also older/

12. SPECIFIC MENTIONS - because I'm pregnant/I only wear seat belts in bad weather/only use when have younger children in carl

13. NEVER USE THEM

14. OTHER

15. DON'T KNOW/NO ANSWER

55. Do you have any children 5 years and under in your household?

Yes $($ To 56$) \ldots \ldots \ldots \ldots \ldots \ldots \ldots$

No $($ TO 57$) \ldots \ldots \ldots \ldots \ldots . \ldots 2$

IF "YES" IN QUESTION 55, ASK:

56. How often are children 5 and under in your household fastened into infant carriers or child safety seats

-- all the time, most of the time, sometimes, or almost never?

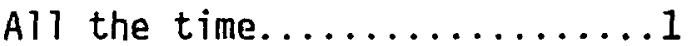

Most of the time...........2

Sometimes...............

Almost never...............4

Don't know/no answer........ 5 
57. What do you believe is the most frequent cause of death among persons under age 35 ? Is it... (READ ALTERNATIVES)

Heart attack...............

Cancer.....................

Other disease............... 3

Alcohol-related traffic accidents...............4

Other traffic accidents.......5

Don't know/no answer..........6

58. How many sigarettes do you smoke a day -- (READ ALTERNATIVES)

Don't smoke cigarettes.........

Less than $1 / 2$ pack...........2

$1 / 2$ to less than 1 pack.......

1 pack or more.............4

59. How often do you visit the dentist

to have your teeth checked?

(READ ALTERNATIVES)

Every 6 months.............

Every year.................

Every 2 years or more.........

Only when something bothers, me. 4

Don't know/no answer.......... 5

60. Do you jog, cycle, swim or exercise regularly at least twice a week?

Yes....................

No......................

61. Within the past three years, have you undertaken any other activities or taken other actions on your own to maintain or improve your health?

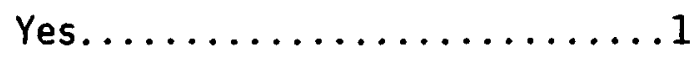

No.....................

Now, just a few final questions for statistical purposes only --

62. What is your age, please?

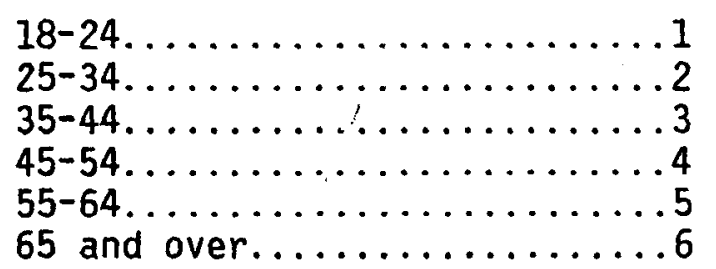


63. What is the last grade of school you completed? (READ LIST)

Less than high school.........1

High school graduate.........2

Some college................ 3

64. Of the following types of work, which one describes what the head of the household does?

(READ LIST)

Self-emp Toyed..............

White-collar..............2

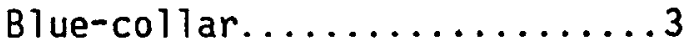

Government worker..........4

Farmer/rancher............. 5

Not in the labor force

(student, house'vife, retired)..............6

Unemployed................ 7

other (SPECIFY)

Don't know/no answer........9

65. Which of the following best describes where you live? (READ ALTERNATIVES)

In a big city..............

In the suburbs around a big

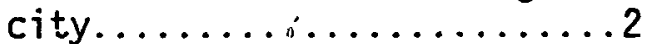

In a small city............

In a town or village........4

In the country............5

Don't know/no answer........6

66. SeX (BY OBSERVATION)

$\frac{\text { IF FEMALE, ASK: }}{\text { employed outside the home? }}$

Male...................

Female/stays at home........

Female/works outside 
APPENDIX B

PANEL

(PANEL)

$(N=201)$

INTERVIEWER

TARRANCE \& ASSOCIATTES

PERSONAL/CONFIDENTIAL

STUDY \#
TIME STARTED
TIME ENDED
FINANCE
CODING

Hello, I'm of Tarrance \& Associates, a national research firm located in Houston. May I speak with ?

As you may recall, we spoke with you a year or two ago about topics relating to automobile safety. We'd like to ask you a few more questions and listen to your opinions as of today.

As you may know, there are many different things we can do to increase auto safety and decrease the number of injuries and deaths which result from auto accidents. Which of the following do you think would be most important in making driving safer? (READ LIST, ROTATE) And which do you think would be the second most important?

1. Less people drinking and driving

Q.1 Most Important Aug. Mar. $1981 \underline{1983}$ $39 \% \quad 67 \% \quad 29 \% \quad 20 \%$

2. More people wearing seat belts

3. Requiring motorcyclists to wear helmets

4. Stricter enforcement of traffic laws

5. Safer cars

6. Don't know/no answer

$\quad 16 \% \quad 16 \% \quad 17 \% \quad 35 \%$

$10 \%$ $2 \%$ $15 \% \quad 12 \%$ $11 \% \quad 10 \% \quad 18 \% \quad 21 \%$ \begin{tabular}{lll}
$17 \% \quad 5 \%$ & $15 \% \quad 12 \%$ \\
\hline
\end{tabular}

$6 \% \quad--\quad 5 \% \quad * \%$

Note: * indicates less than $1 \%$. 
Now I'd like to get your opinions on several different issues relating to seat beits. First -

3. How often do you wear seat belts when roads are wet, or snow and ice covered? (REF.D ALTERNATIVES)

Aug. Mar. $1981 \underline{1983}$

Always..................... Most of the time.............2

Sometimes...................

Never....................4

Don't know/no answer..........
$32 \% \quad 34 \%$

$11 \% \quad 14 \%$

$19 \% 20 \%$

$36 \% 31 \%$.

4. How often do you wear seat belts on

long trips? (READ ALTERNATIVES)

Always.................. $35 \%$

Most of the time............. $11 \%$

Sometimes................ $18 \%$

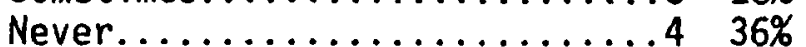

Don't know/no answer.........5

$36 \%$

$16 \%$

$18 \%$

$27 \%$

$1 \%$

5. How often do you wear seat belts on

short trips around town? (READ

ALTERNATIVES)

Aiways (TO 7)............ 24\% 24\%

Most of the time (TO 6).......2 $4 \%$ 9\%

Sometimes (TO 6)............. $17 \% \quad 17 \%$

Never (TO 6) ............. 55\% 49\%

IF "MOST OF THE TIME", "SOMETIMES", OR "NEVEF" IN 0.5, ASK:

Do you agree or disagree with the following statement --

6. "There's nothing anyone can do that would make me use seat belts all of the time."

IF CHOICE MADE, ASK: And do you feel strongly about

Agree/strongly............ $22 \%$

Agree..................... $10 \%$

Unsure (DO NOT READ) ........... $7 \%$ that?

Disagree...................... $30 \%$ 29\%

Disagree/strongly.......... $31 \% \quad 31 \%$ 
And do you agree or disagree with the following statements --

7. "I'm quite sure I won't be in an autio accident because I drive defensively." (PROMPT: DO YOU AGREE OR DISAGREE WITH THIS STATEMENT?)

IF CHOICE MADE, ASK:
do you feel strongly about
that?

Agree/strongly.............

Agree.................

Unsure (DO NOT READ) .........

Disagree................ 4

Disagree/strongly......... 5

8. "Wearing seat belts is like many other good health practices such as brushing your teeth and having regular medical check-ups." (PROMPT: DO YOU AGREE OR DISAGREE WITH THIS STATEMENT?)

IF CHOICE MADE, ASK: And do you feel strongly about

Agree/strongly.............

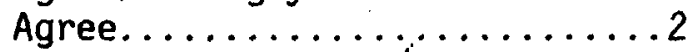

$(58 \%)$

Unsure (DO NOT REACI) ......... that?

Disagree................ 4

9. "People think they won't be in an accident because they are safe drivers in control of their car." (PROMPT IF NECESSARY)

IF CHOICE MAOE, ASK:
do you feel strongly about
that?

Agree/strongly............1 Agree.................... Unsure (DO NOT READ) ......... Disagree............... Disagree/strongly..........5

10. "People are more likely to put their seat belts on if they think that driving conditions are more dangerous than usual." (PROMPT IF NECESSARY)

IF CHOICE MADE, ASK:
do you feel strongly about
that?

Agree/strongly.............. Agree.................... Unsure (DO NOT READ)......... Disagree................. 4 Disagree/strongly.......... 5 
11. "I don't think seat belts work properly when they feel so loose around me when I fasten them."

(PROMPT IF NECESSARY)

IF CHOICE MADE, ASK:
do you feel strongly about
that?

Agree/strongly...............

Agree....................

Unsure (DO NOT READ) .........

Disagree...............4

Disagree/strongly.......... 5

Now I'd like to read you some statements about seat belts made by two people. Please tell me which one comes closest to your own opinion --

12. Mr. Jones feels that he can fulfill his responsibility to passengers in his car by asking them to fasten their seat belts and by driving carefully.

Mr. Smith feels that he can fulfill his responsibility to passengers in his car by making sure that they are covered under his auto insurance policy.

Who comes closest to your own opinion

-- Mr. Jones or Mr. Smith?

$\frac{\text { IF CHOICE MADE, ASK: }}{\text { you feel strongly about that? }}$

Mr. Jories/strongly...........

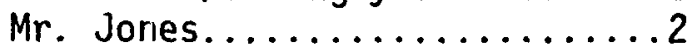

Unsure (DO NOT READ) .........

Mr. Smith...................

Mr. Smith/strongly..........

13. Mr. Jones feels that he can fulfill his responsibility to passengers in his car by asking them to fasten their seat belts and by driving carefully.

Mr. Smith never asks passengers in his car to fasten their seat belts because he feels he would be imposing on them if he did so.

Who comes closest to your own opinion -- Mr. Jones or Mr. Smith?

$\frac{\text { IF CHOICE MADE, ASK: }}{\text { you feel strongly about that? }}$

Mr. Jones/strongly...........

Mr. Jones.................

Unsure (DO NOT READ) .........

Mr. Smith...............

$\mathrm{Mr}$. Smith/strongly.........5 
14. Mr. Jones feels that the car's buzzer is a reminder to him to put on his seat beit.

Mr. Gray on the other hand just ignores the buzzer when he gets into his car and doesn't put his belt on.

Who comes closest to your own opinion -- Mr. Jones or Mr. Gray?

$\frac{\text { IF CHOICE MADE, ASK: }}{\text { you feel strongly about that? }}$

Mr. Jones/strongly............

Mr. Jones..................

Unsure (DO NOT READ)..........

Mr. Gray...................

Mr. Gray/strongly...........

15. Mr. Jones thinks that if he were in a serious auto accident and were using a safety belt his injuries would be less than if he wasn't wearing a safety belt.

Mr. Smith on the other hand feels that if he were in a serious auto accident and was wearing a safety belt his injuries would be more severe, therefore he doesn't wear his safety belt.

Who comes closest to your own opinion -- Mr. Jones or Mr. Smith?

$\frac{\text { IF CHOICE MADE, ASK: }}{\text { you feel strongly about that? }}$

Mr. Jones/strongly...........

Mr. Jones..................

Unsure (DO NOT READ) .........

Mr. Smith................4

Mr. Smith/strongly..........5

On another matter --

16. Mr. Johnson feels that it takes too much time and is too hard to remember to fasten his seat belt every time he sits down in his car, so he rarely uses them.

Mr. Davis feels that putting on seat belts is just like brushing his teeth or watching his diet, and it is worth the slight inconvenience it takes to fasten his seat belts every time he sits down in his car.

Who comes closest to your cwn opinion

-- Mr. Johnson or Mr. Davis?

IF CHOICE MADE, ASK:
you feel strongly about that?

Mr. Johnson/strongly..........

Mr. Johnson...................

Unsure (DO NOT READ)..........

Mr. Davis................

Mr. Davis/strongly..........5 
17. Mr. Cooper does not wear seat belts because he thinks he is a good driver and won't be in an accident.

Mr. Lewis wears his seat belts because he isn't sure about the other drivers and just doesn't like to take chances.

Who comes closest to your own opinion -- Mr. Cooper or Mr. Lewis?

IF CHOICE MADE, ASK:
you feel strongly about that?

Mr. Cooper/strongly..........1

Mr. Cooper..................

Unsure (DO NOT READ)...........

Mr. Lewis.................

Mr. Lewis/strongly........... 5

And --

18. Mr. Thompson feels he has much less control over himself and his car when he drives with his seat belts on.

Mr. Evans feels he has much more control over himself and his car when he drives with his seat belts on.

Who comes closest to your own opinion

-- Mr. Thompson or Mr. Evans?

IF CHOICE MADE, ASK:
you feel strongly about that?

Mr. Thompson/strongly........1

Mr. Thompson...............

Unsure (DO NOT READ).........

Mr. Evans................

Mr. Evans/strongly.........5

On another matter --

19. How much control do you feel you have in preventing your own involvement in automobile accidents? Do you feel you have... (READ ALTERNATIVES)

Almost total control..........1

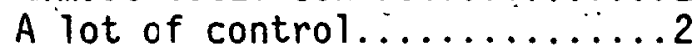

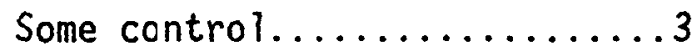

Very little control..........4

Unsure (DO NOT READ).........5 
20. If you were involved in an auto accident, what do you feel would be the most likely cause? Would it be... (READ ALTERNATIVES)

1) Your own driving error

$\begin{array}{r}3 \% \\ \hline 23 \% \\ \hline 42 \% \\ \hline 1 \% \\ \hline 4 \% \\ \hline 8 \% \\ \hline 15 \% \\ \hline 2 \% \\ \hline\end{array}$

2) A drunk driver

3) Someone else's driving error

4) A mechanical defect in the car

5) Unavoidable bad luck

6) Poor road conditions

7) Poor weather conditions

8) Don't know/no answer

01. LOWER SPEED LIMIT - just lowering the speed limit - that's the only reason/stower speed limit/lowering the speed limit did a lot of good/because of the drop in the speed limit/

02. 55 M.P.H. SPEED LIMIT - 55 m.p.h. limit is a good measure/reduction in speed from 65 to $55 /$ speed limits not going over 55 m.p.h./ 55 m.p.h. has cut down the death rate/55 m.p.h. speed limit has caused people to have less accidents/

03. DRUNK DRIVING LAWS - I would say the stricter alcohol laws/laws on drunk drivers are stricter here/the new alcohol laws against driving drunk/tougher drunk drivers laws/

04. CRACK DOWN ON DRUNK DRIVERS - cracking down on drunk drivers/ there is more of a watch on drunk drivers/cracking down on driving while drinking/more strict on drunk driving/

05. ENFORCEMENT OF DRUNK DRIVING LAWS - stricter enforcement on drunk drivers/enforcement of the D.W.I. laws/stronger police enforcement of drunk driving/

06. FEWER DRUNK DRIVERS/DRUGGED DRIVERS - there wasn't as many drunk drivers on the road/less drinking and driving/getting the drunk drivers off the road/people are smoking less pot/

07. PUBLIC CONCERN/DRUNKEN DRIVING - more talk about drinking and driving/an awareness of the public to drinking while driving/ people are cutting down on driving after having too much to drink/the crusade against drunken drivers/drunk driving groups, like M.A.D.D.I 
Question 21 continued

08. SAFETY BELTS/CAR SEATS - people are using their seat belts more than they used to/I have been seeing a lot nore people using their seat belts/because of seat belts/the news telling people to wear safety belts/infant car seats required by law/

09. MORE LAW ENFORCEMENT/ENFORCEMENT OF 55 M.P.H. LIMIT - there's been more law enforcement/police enforcement of the regulations/ starting to control traffic better/stricter enforcement of driving laws/enforcement of the speed $1 \mathrm{imit} / \mathrm{cops}$ are giving more tickets/tighter enforcement of the $55 \mathrm{~m} . \mathrm{p} . \mathrm{h}$. speed $\mathrm{limit/}$

10. THE ECONOMY - with more people unemployed, they are unable to buy gas/economics, no money to drive/less people on the road now because of money/it costs more to get crashed cars fixed/ people don't have money to take trips anywhere/

11. PRICE OF GAS/GAS SHORTAGE - price of fuel, people can't afford to drive as much/people can't afford the gas/people are out of gas because of gas being so high/the lack of gas/people drove conservative to save gaș/

12. REDUCED TRAFFIC/LESS DRIVING - less density of cars on the road/ people aren't driving as much because of the oil shortage/people are driving less/people driving less and putting less miles on the car/car pooling/

13. DRIVERS ARE CAREFUL - people drive more defensively/people are more careful and are safer drivers/people are becoming more alert/ people are more careful now because cars are becoming more expensive to $f i x /$

14. PEOPLE ORIVE SLOWER - people driving slower speeds, more accident consciousness/people are driving a little bit slower/people travel at lower speeds/

15. PUBLIC AWARENESS/EDUCATION - people taking defensive driving/better education of drivers/a lot of national publicity about safer driving/people benefit from hearing about accidents/more awareness of driving habits/people are more aware of safety/more awareness of driving hazards/public awareness to accidents has improved significantly/

16. SAFER CARS - making the automobile safer/smaller engines in the cars/concentration on safety in cars/cars have been built safer/ safer cars, there is a lot less old junk on the roads/all the new cars coming in with new automatic safety belts that flip on with the doorl

17. STRICTER LAWS - stricter traffic laws/tough laws/stricter laws being imposed/pushing more safety regulations/the new law/tougher laws about speeding and so forth/more speeding laws slowing up trucks/stiffer penalties/ 


\section{Question 21 continued}

18. WEATHER/ROAD CONDITIONS - we had a milder winter/not as much ice and snow/road conditions/bad roads, lower speed/snow falling more and less people driving/

19. INSURANCE REQUIREMENTS - insurance requirements/the large demand

20. OTHER

21. DON'T KNOW/NO ANSWER

22. Do you drink alcoholic beverages -even occasionally -- or are you a total abstainer?

Yes (CONTINUE) .............

Total abstainer (TO 34 )......

23. Think for a moment about your own personal driving habits over the past year. Trying to be as honest as you can, how frequently would you say you drive within one hour of drinking an alcoholic beverage? (READ ALTERNATIVES)

Daily.....................

2 to 6 times a week...........2

Once a week.................

Once every two weeks.........4

Once a month...............

About 4-6 times a year........6

About once or twice a year.....7

Never (ASK 24 ONLY, THEN

SKIP TO 33$) \ldots \ldots \ldots \ldots \ldots . . .6$

24. And, one year ago, how frequently would you say you drove within one hour of drinking an alcoholic beverage? (READ RESPONSES)

Daily....................

2 to 6 times a week..........

Once a week................ 3

Once every two weeks.........4

Once a month.............. 5

About 4-6 times a year.......6

About once or twice a year....7 
25. And would you say your frequency of drinking within one hour of driving has changed significantly over the past year?

Yes (TO 26)................

Unsure (TO 27) .............

IF "YES" IN QUESTION 25, ASK:

26. And what are one or two reasons why you feel that way?

(PROBE: PLEASE TELL ME MORE ABOUT THAT)

01. STRICTER D.W.I. LAWS - our stiffer drunk driving laws/ laws are tougher/the new D.W.I. law that enforces 48 hours in jail/they are cracking down on laws for drunk drivers/

02. DON'T ORINK AS MUCH - I slowed down a lot on my drinking/

I have cut down a lot on my drinking/I consume much less alcohol/I am drinking less than I used to/

03. MORE RESPONSIBILITY - more responsibility, I am thinking of the safety of myself and my family/I am more safety conscious, I've got a child now/care about my kids/

04. DON'T GO OUT AS MUCH - I don't go out as much, I usually drink at home/I don't go much anymore/married and didn't party as much/we don't go out very often/my social activities have become less/

05. MORE AWARE - I am more aware of the effects of drinking and driving/I'm more aware of it being dangerous/awareness of drunk drivers/

06. DON'T DRINK AND DRIVE - don't drink and drive that much/I don't believe in drinking and driving/don't drink as often just before driving/I just have my wife drive me/I try not to drink when I'm driving/

07. HAVE BEEN CAUGHT - got caught drinking and driving/I don't have a license and I have been arrested for drunken driving/I had a close call/

08. MONEY - my income has changed significantly/money/I can't afford to go out to clubs or parties as much anymore/ 
Question 26 continued

09. GO OUT MORE OFTEN - I go out more often/more social occasions/go to more social affairs and different functions/

10. DO IT MORE NOW - I do it more now/I've been drinking lately/

11. LIFE STYLE - because of my life style/changed my life style/

More than once $a$ week..........

About once a week..............

One or two times a month.......3

Less than once a month........4

One or two times a year.......5

Never................. 6

27b. Restaurants

(PROMPT IF NECESSARY)

More than once a week......... 1

About once a week..............

One or two times a month.......3

Less than once a month.......4

One or two times a year.......5

Never..................6

27c. Cocktail lounges

PROMPT: HOW FREQUENTLY HAVE YOU

PERSONALLY HAD TO DRIVE HOME FROM

A COCKTAIL LOUNGE AFTER DRINKING

WITHIN ONE HOUR --

More than once a week.........

About once a week...........2 
27d. Other people's homes (PROMPT IF NECESSARY)

More than once a week.........1

About orice a week............2

One or two times a month.......3

27e. Private party held in a public place, such as a hotel or banquet hall. (PROMPT IF NECESSARY)

More than once a week.........1

About once a week...........2

One or two times a month.......3

Less than once a month........4

One or two times a year.......5

Never..................6

27f. Sporting events

(PROMPT IF NECESSARY)

More than once a week.......... I

About once a week...........2

One or two times a month.......3

Less than once a month........4

One or two times a year........5

Never...................6

27g. Drinking in my car (PROMPT IF NECESSARY)

More than once a week.........1

About once a week...........2

One or two times a month.......3

Less than once a month........4

One or two times a year.......5

Never.................6 
28. Think for a moment about a situation in which you drive within one hour of having had too much to drink. In your own estimation, if you did this 100 times, how many times would you be caught and punished?

(RECORD EXACT RESPONSE)

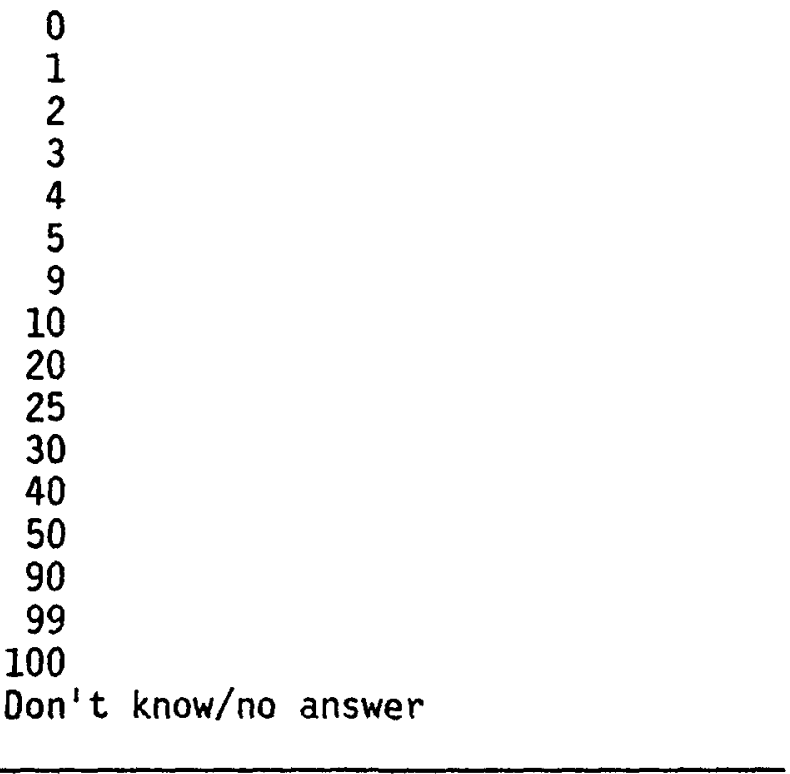

29. How high would the number have to be

in order for you to decide not to

drive after having had too much to

drink?

(RECORD EXACT RESPONSE)

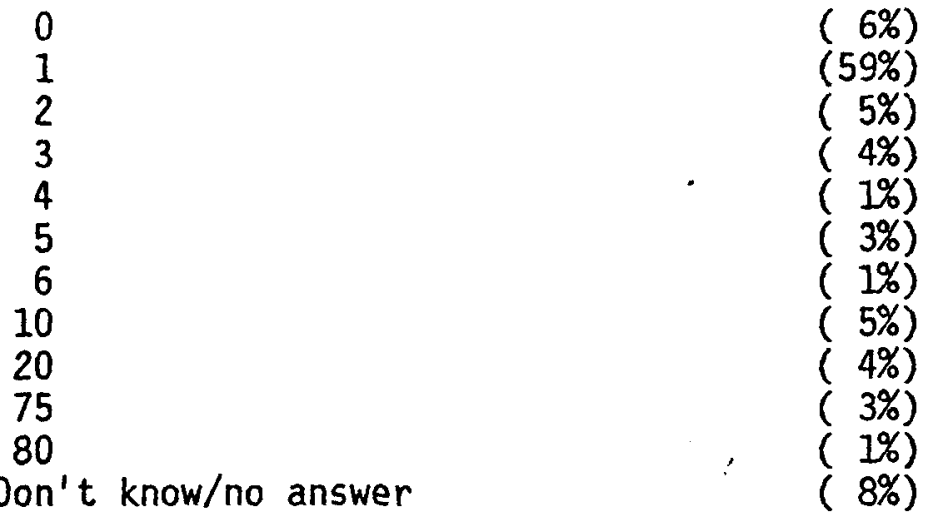


30. Have you personaliy made any efforts to reduce the number of times you have driven after drinking too much in the past 6 months?

Yes (TO 31) ................

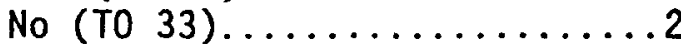

Don't know.................

IF "YES" IN QUESTION 30, ASK:

What have you done? (DO NOT READ, CHECK EACH THAT APPLIES)

(FOR EACH MENTIONED, ASK: Is that something you have started to do recently? RECORD BELOW IN THIRD AND FOURTH COLUMNS FOR EACH ACTION MENTIONED. )

\begin{tabular}{|c|c|c|c|}
\hline \multicolumn{2}{|c|}{ Q. 31} & \multirow{2}{*}{$\frac{0.32}{\text { Recently }}$} & \\
\hline Mentionec & $\begin{array}{c}\text { Not } \\
\text { Mentioned }\end{array}$ & & $\begin{array}{l}\text { Don't } \\
\text { Know }\end{array}$ \\
\hline
\end{tabular}

a) Con't drink alcoholic beverages

\begin{tabular}{|c|c|c|c|c|}
\hline $10 \%$ & $90 \%$ & $6 \%$ & $3 \%$ & $90 \%$ \\
\hline $39 \%$ & $61 \%$ & $23 \%$ & $16 \%$ & $61 \%$ \\
\hline $6 \%$ & $94 \%$ & $3 \%$ & $3 \%$ & $944 \%$ \\
\hline
\end{tabular}

d) Drink some alcoholic beverages and some non-alcoholic beverages

e) Stop drinking at a predetermined time

f) Had someone else drive nome

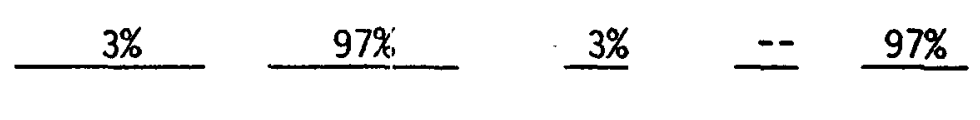

b) Limit the number of drinks

c) Eat food

$$
6 \%
$$

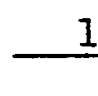

g) Took a taxi/public transportation

$13 \%$

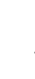

$87 \%$

$6 \% \quad 6 \% \quad 87 \%$

$32 \%$

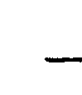

$68 \%$

$19 \%$

$13 \% \quad 68 \%$

$3 \%$

h) Test self for own alcohol level

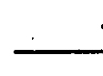

i) Stayed overnight

j) Drank coffee

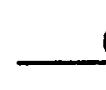

k) Diluted drinks

1) Other (SPECIFY)

\begin{tabular}{ccccc}
$\frac{6 \%}{10 \%}$ & $\frac{94 \%}{90 \%}$ & $\frac{3 \%}{6 \%}$ & $\frac{3 \%}{3 \%}$ & $\frac{94 \%}{90 \%}$ \\
\hline-- & $\frac{100 \%}{23 \%}$ & $\frac{77 \%}{16 \%}$ & $\frac{--}{100 \%}$ & $\frac{77 \%}{6}$ \\
\hline
\end{tabular}


33. How can you tell when you have had too much to drink to drive safely? (DO NOT READ, RECORO RESPONSES ONLY -- DO NOT PROBE)

\begin{tabular}{|c|c|c|c|}
\hline & & Mentioned & $\begin{array}{c}\text { Not } \\
\text { Mentioned } \\
\end{array}$ \\
\hline a) & Number of drinks & $11 \%$ & $89 \%$ \\
\hline b) & Slurred speech & $3 \%$ & $97 \%$ \\
\hline c) & Weaving/staggering: & $12 \%$ & $88 \%$ \\
\hline d) & $\begin{array}{l}\text { Personality change, such as very } \\
\text { talkative, loud, aggressive, very } \\
\text { quiet, etc. }\end{array}$ & $7 \%$ & $93 \%$ \\
\hline e) & Can't stand up & $1 \%$ & $99 \%$ \\
\hline f) & STeepy & $3 \%$ & $97 \%$ \\
\hline g) & General behavior & $14 \%$ & $86 \%$ \\
\hline h) & Passed out & $\star \%$ & $100 \%$ \\
\hline i) & Can just tell (nothing specific) & $10 \%$ & $90 \%$ \\
\hline j) & Can't tell & $5 \%$ & $95 \%$ \\
\hline k) & Dther (SPECIFY) & $14 \%$ & $86 \%$ \\
\hline
\end{tabular}

I am going to read you a statement. Please indicate whether you agree or disagree --

34. "Except for alcoholics and persons with real drinking problems, few people drive after drinking too much to drive safely."

$\frac{\text { IF CHOICE MADE, ASK: }}{\text { you feel strongly about that? }}$

Agree/strongly.............

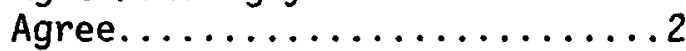
Unsure (DO NOT READ) ......... Disagree................. 4 Disagree/strongly......... 5

35. About how many miles did you personally drive during the past 12 months? 
36. About how many miles do you drive in an average week day (Monday-Friday)?

$0-10 . \ldots \ldots \ldots \ldots \ldots \ldots \ldots .1$

$12-29 . \ldots \ldots \ldots \ldots \ldots \ldots \ldots . . . . .2$

(27\%)

$30-69 . \ldots \ldots \ldots \ldots \ldots \ldots \ldots . . . .3$

70 and over...............

Don't know/no answer......... 5

37. About how many miles do you drive on an average weekend (Saturday-Sunday)?

$0-10 \ldots \ldots \ldots \ldots \ldots \ldots \ldots . . .1$

$11-20 \ldots \ldots \ldots \ldots \ldots \ldots \ldots . .2$

$21-50 \ldots \ldots \ldots \ldots \ldots \ldots \ldots . . . .3$

Over $50 \ldots \ldots \ldots \ldots \ldots \ldots \ldots .4$

Don't know/no answer..........5

38. How many automobile trips have you taken in the last year that were over 200 total miles?

$0-1 \ldots \ldots \ldots \ldots \ldots \ldots \ldots \ldots \ldots$

$2-4 \ldots \ldots \ldots \ldots \ldots \ldots \ldots \ldots \ldots .2$

5 and over................

39. Is this more or less than the year before?

More...................

Unsure (DO NOT READ)..........

No difference (DO NOT READ)....3

Less.................... 4

40. In what month and year was the last trip you took which was over 200 total miles?

Year of Last 200 Mile Trip

Before 1980

1980

1981

1982

1983

Don't know/no answer

$\begin{array}{r}6 \% \\ \hline 5 \% \\ \hline 11 \% \\ \hline 39 \% \\ \hline 29 \% \\ \hline 9 \% \\ \hline\end{array}$

Season of Last 200 Mile Trip

\section{Fall/Winter}

Spring/Summer

Don't know/no answer
$54 \%$

$35 \%$

$\underline{11 \%}$

41. How many people were in the car on that trip?

$1 . \ldots \ldots \ldots \ldots \ldots \ldots \ldots \ldots \ldots \ldots$

$2 \ldots \ldots \ldots \ldots \ldots \ldots \ldots \ldots \ldots \ldots \ldots, 2$

$3 \ldots \ldots \ldots \ldots \ldots \ldots \ldots \ldots \ldots \ldots \ldots$

4 or more...................... 4

Don't know/no answer. 
42. Do you think you and your family drove more in 1982 than you did in 1981, or less in 1982 than you did in 1981 ?

More $($ TO $43-1) \ldots \ldots \ldots \ldots . \ldots 1$

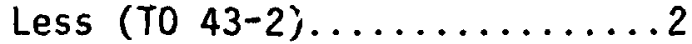

The same (TO 44)............

Unsure (DO NOT READ) (TO 44 )...4

IF "MORE" IN QUF'STION 42, ASK:

43-1 And why did your family drive more in 1982?

01. VACATION - because of our whole family going on vacation/ we took a vacation/we went on our vacation/we just drove on vacation instead of flying/took vacation to another state/

02. MORE/LONG TRIPS - took more trips/we went on a lot of trips/ took long trips in 1982/we took a $6000 \mathrm{mile}$ trip/more frequent short trips/

03. BUSINESS TRIPS - mostiy business trips/I took the family with me more on business trips/my business required it/ working out of town now/more business-related trips/

04. VISITING FAMILY/FRIENDS - our son moved to Kansas and we go to see him quite a lot/more relatives that live further away/my children have moved to different states and I go to visit them/we visited more friends/

05. ILLNESS/DEATH - we had to drive 120 miles to see my motherin-law in the hospital/because of illness/lot of sickness in immediate family/had to make several trips to the hospital/had death in family, needed to travel/

06. MORE PLACES TO GO - we had more places to go/went more places last year/we went different places/going to more places now than in past years/

07. MORE TIME - had more time off/we had more time to travel/ more time off from the job/my husband retired and we had more time to go places/have more time to travel/

08. LOWER GAS PRICES - the lowering of gas prices in 1982/gas was little bit cheaper last year/the price of gasoline went down/ 
Question 43-1 continued

09. MORE DRIVERS IN FAMILY - we had more drivers in the family/ we have a third driver, our son got his license/more people ir the: family drive now/

10. MORE MONEY - we made more money/we could afford it/had more money to travel/financially better off/I went back to work and we had more money/;

11. BOUGHT SECOND CAR - we acquired another car/we got another car/we bought an additional car/we bought a second car/

12. COLLEGE - taking kids back and forth to college/my daughter went off to college/more trips to college/I went to school 30 miles away every day that year/

13. CHILDREN OLDER/MORE ACTIVITIES - kids are oider and they go more places/kids are older and have a greater demand for transportation/we were involved in more youth activities/

14. MORE TO DO - more things to do/more family gatherings/doing more things/family things coming up/

15. MORE FAMILY MEMBERS - my family was larger then/more working members in the family/more of us/there were more people in the family/more family members/

16. MORE OPPORTUNITIES - more opportunity to travel/more opportunities and invitations to do more things/we had more opportunity to go/we just had more social engagements than the year before/.

17. CAMPER/MOTOR HOME - we have a camper now/we bought a motor home and have taken more vacations/we bought a motor home ...we are seeing the U.S./

18. WIFE/CHILDREN WORKING - my wife took a job and had to drive about 70 miles per day/because my kids have jobs/

19. MORE ERRANDS/VOLUNTEER WORK - I have more errands to run than I used to/more running around/more volunteer work/

20. CAR POOL - my children go to Catholic school and I have to drive them/I drive a car pool, drive children to school 10 miles away/

21. JUST HAPPENED THAT WAY - it just happened that way/it was just the way things happened/just coincidence/

22. OUT OF COUNTRY IN 1981 - in 1981 we lived in Korea and weren't many places to drive/we were in England in ' $81 /$

23. DON'T KNOW/NO ANSWER 
IF "LESS" IN QUI:STION 42, ASK:

43-2 And why did your family drive less in 1982?

01. GAS PRICES/AUTO EXPENSES - cost of gas/the gas price going up/high-priced gas/I'm cutting back on use of the car because of the high cost of gas and maintenance of the car/ cost of operating a vehicle/we can't afford the gas/

02. TOOK FEWER/NO TRIPS - we took less long distance trips/ took less family trips/we haven't taken the trips that we had previously/didn't take any trips/we went several places the year before/we took a long trip in ' 81 and didn't in ' $82 /$

03. ECONOMY - the high prices of everything/with the economy Tike it is, we just couldn't afford to/cost, expensive for food, gas and lodging/the money was tighter/the economy/

04. ELDERLY/RETIRED - we're getting older and don't have the energy/I'm 70 years old, so I don't drive as much as I used to/I'm retired and I can't see too well/we're both retired now and don't have to drive to work/

05. NO/LESS MONEY - no dough, no go/there was no money for gasoline/income was. lower/ain't got no money to go nowhere/we had a lot less money in 1982/no money/

06. NO VACATION/LESS VACATION TIME - didn't take a vacation/ couldn't afford a vacation/we had less vacation time last year/a combination vacation was planned closer to home/ I didn't have as much vacation time/

07. DIDN'T GO ANYWHERE/NOWHERE TO GO - we just didn't have any places to go/we had no reason to go any place/just don't go much anymore/just don't go anywhere/just sit home a lot/

08. PREFER NOT TO/PERSONAL LIFE STYLE - lack interest, didn't go anywhere/didn' $t$ feel like going anywhere/life style/ cut down on driving, I'm tired of driving/I didn't choose to drive as much/we got in a rut and $d^{2} n^{2} t$ take off on weekends as much/

09. FAMILY ILLNESS - family illness/my husband was in the hospital/I was taking care of my mother and she was very sick/my husband was injured at work/my wife is not well/ I was taking care of a sick aunt/

10. NO TIME/BUSY WORKING - we both had jobs and were working and didn't have time to take a trip/I was too busy to go anywhere/lack of time/no free time/ 
Question 43-2 continued

11. ALTERED WORKING CONDITIONS - because of changing working conditions/husband worked different hours/it was harder for me to get out of work/my girl friend don't have weekends off anymore/schedule of my job/

12. USE TRAIN/PLANE - we have more money and fly more/our vacation was different, we took a plane in $182 /$ I go on the train a lot now/we fly most of the time/we take a train or plane/

13. UNEMPLOYED - we were unemployed in the year 1981/I quit work so I don't leave the house as much/I was laid off from work/husband was unemployed/

14. CAR TROUBLE/NO CAR - we had trouble with the car/I don't have a car so I only drive a rental car or a borrowed car/ our car gets bad mileage/my car was getting old/the family owned less vehicles in 1982/

15. DIDN'T VISIT RELATIVES - I didn't go and visit my relatives as much/I didn't go visit my son who lives about 400 miles from here round trip/we did not have the opportunity to visit family as much/

16. PERSONAL CIRCUMSTANCES - personal reasons/I don't drive anymore/it was a complicated year, we had a lot of personal problems/I was overseas in 1982/we got a pool so we don't go anymore/

17. SCHOOL-RELATED MENTIONS - my daughter started college/I am working for a master degree and am really saving/my wife is in school/my son has graduated from college so we have stopped making the trips to see him/

18. FAMILY CHANGES - I got divorced/in 1981 I was pregnant/my wife was pregnant at the time/new baby, we were not able to move around much/my father passed away/I lost my wife in ' 82 and $I$ don't feel like going places anymore/

19. RIDE SHARING/FUEL CONSERVATION - I car pool now/rearranged work schedule where my wife and I ride together/I share my driving with other people/my son-in-law drives us on trips/ we're conserving on gas/

20. NO REASON

21. OTHER 
44. In general, have your driving habits -trip length, where you drive, when you drive, how you drive -- changed in the last year?

Yes (TO $45 \& 46) \ldots \ldots \ldots \ldots \ldots . . . . .1$ Unsure (DO NOT READ)(TO 47) ...2

IF "YES" IN QUESTION 44, ASK:

45. In what way have your driving habits changed?

01. MORE CAUTIOUS - I've become more cautious/I am a lot more careful/I'm more coservant and cautious than I used to be/ more precaution toward other drivers now/I'm more cautious of people around t/s/

02. DRIVE SLOWER - I have slowed down more/slowed way down/ drive slowTy, more content with the speed limit/I don't drive as fast as I used to/

03. DRIVE LESS - I drive less/I'm not driving as much as I was/don't drive as much because don't work much/I've joined a car pool so don't drive to work everyday now/

04. DEFENSIVE ORIVING - driving more defensively/more defensive driver/we moved into a new area and have to drive more defensively/try to drive more defensively/

05. DRIVE MORE - I'm driving more/I drive a lot more/I am driving more highway miles than a year ago/

06. USE SEAT BELT - I use the seat belt always now/started wearing seat belts/I use seat belts more/

07. AVOID DRINKING - less frequency of drinking/I don't drink at all anymore/stop drinking and driving/avoid driving when I drink/don't drink anymore/

08. SAFETY CONSCIOUS - improved safety habits/I'm more conscious of safety/took a defensive course class and now am more aware of my driving/

09. OLDER/MORE CAREFUL - getting older makes you more careful/ when you get older you drive a little different/

10. AFFECTED BY WEATHER CONDITIONS - I refuse to drive on ice anymore/I'm more careful in weather conditions now/dictated by the weather/if the weather is bad, I don't drive/

11. SHORTER TRIPS - shorten my trips, get more things when I go to town/do more errands in one trip/I try to take as little number of trips as possible/ 
Question 45 continued

12. NOT DRIVING - not driving/I didn't like driving so I let my Ticense expire/I don't have the ability to drive anymorel

13. LONGER/MORE TRIPS - only use it for long trips/longer trips/ my husband retired and we take more trips in the country/

14. MORE DAYTIME DRIVING - more daytime driving/I changed from driving at night to day/we try not to drive at night/

15. NERVOUS/TENSE - I am more nervous to get on the highway/I notice when you get tense it affects your driving/

16. OTHER

17. DON'T KNOW/NO ANSWER

IF "YES" IN QUESTION 44, ASK:

46. And why did you change your driving habits?

01. WORK RELATED - change in occupation/my new job is closer for me/my employment is further away now/more business so we need to drive more/

02. SCHOOL RELATED - I started back to school/I go to a closer school now/because I'm no longer in school/

03. CHILDREN - I have had children/young children to protect/ my children are very important to me/our children'

04. MATURITY/WISDOM - just wised up/that goes with age/I became more settled and got older/it comes with age/you just get older and wiser/

05. OLD AGE/RETIRED - just getting to old/because we have retired/because we're. old/as I get older, my responses diminish/

06. COST/ECONOMY - no money/cost factors/more car pooling/for economic reasons/the economy/bad economy condition/

07. ACCIDENT/SELF - I had an accident/I had a wreck on my motorcycle/wrecked two Corvettes and one other car/I have realized after being in an accident just how many turkeys are out there/I had an accident and learned from it/ 
Question 46 continued

08. AWARE/AVOID ACCIDENTS - seen too many accidents/more aware

of the accidents/so many accidents you hear about/trying

to avoid accidents/had a few close calls lately/

09. OTHER DRIVERS - more drunk drivers on road/the people don't seem to look where they're going/to protect myself from those aggressive drivers/more bad drivers on the road now/ drive for yourself and other person too/

10. GOT CAUGHT - I got a speeding ticket/I got so many tickets and lost my license two times/my last DWI got my attention/ I was very embarrassed about being arrested/

11. LAW CHANGES - speed limit change/the new laws/they raised the ticket prices/tougher laws on drunk drivers/

12. INCREASED PUBLICITY/AWARENESS - all the programs about drunk driving/knowladge through publicity/more awareness of possible outcomes/I'm more aware, the whole public is more aware/

13. TRAFFIC - more traffic/too many cars on the highway/don't Tike the traffic congestion/

14. SPECIFIC PRESENTATION - defensive driving class/because of that telephone call a year ago about seat belts/in that class I saw the bad points of my driving/saw a film on seat belt use/

15. TO STAY ALIVE - just to stay alive/I want to live/was putting my life in jeopardy/

16. DRIVE MORE/MORE TRIPS - more long distance trips/I drive morel

17. DON'T DRIVE MUCH - I hate driving/never did drive that much/ don't drive anymore/don't need to drive/easier to ride the bus/

18. PERSONAL/FAMILY REASONS - I have care of my mother who is iा1/I have been sick/my children are in school and I have more time/I began staying home and raising a family rather than working/I was saved/

OTHER

20. DON'T KNOW/NO ANSWER 
47. Now think back to the period between Thanksgiving and New Year's -- last year, that is, 1982. Did you use your car more than during the previous holiday season, or less than during the previous holiday season?

More (TO 48-1)..............

Less (T0 48-2)..............2

The same (TO 49)............

IF "MORE" IN QUESTION 47, ASK:

48-1 And why did your family use your car more this holiday season than the previous one?

01. VIOITTNG RELATIVES - we went to visit my sister more/my daughter lives out of state/we went to visit family/we visited relatives more/our family lives farther away/

02. TOOK A TRIP - because we took a trip to Indiana/we took a trip to Georgia during the holidays/we traveled East for Christmas/went out of town/

03. VISITING/VISITING FRIENDS - we did more visiting this holiday season/we went visiting with holiday guests/more people to see/we were visiting friends/some of our friends moved close enough to drive tol

04. MORE PLACES TO GO - more places to go/there was more to do this year/we had more parties to attend/more to do/because we went out more to different places/

05. JOB RELATED - I got a part-time job/I had to work/I have a new job and drive to Jersey City every weekend/I was further from my job last season/

06. SCHOOL RELATED - I was in school and was driving back and forth/because I go to school/more college trips/

07. MEDICAL RELATED - because I went to the doctor more/because my wife was hospitalized and I was going back and forth to see her/

08. BETTER WEATHER - the weather was nicer so we went on more trips/the weathe: was better/

09. OUT-OF-TOWN GUESTS - we had out-of-town company and went out more/because we had out-of-town guests and we took them out showing them around/my sister was visiting and went to take her sightseeing/

10. MORE/BETTER CARS - we had an additional car to use/better carlour car was working this season/we only had one car and now we have two, so we're both driving/ 
IF "LESS" IN QUESTION 47 , ASK:

48-2 And why did your family use your car less this holiday season than the previous one?

01. STAYED HOME - we didn't go any place/we stayed home this year/just didn't/I just don't go anywhere/family stayed home more this year/we just celebrate at home/

02. OTHERS CAME HERE - company came over/everybody came to our house for the holidays/my mother came here instead of our going there/it was my turn to have everyone/rotating family Christmas/

03. TOO EXPENSIVE - we were broke/cutting back to save money/ money shortage/too expensive/the poor economic situation/ less money to go places/

04. STAYED LOCAL - we didn't go away on a trip/I stayed in the local area/didn't go out of town/we just went a block/we just stayed with neighbors/

05. FAMILY ILLNESS/ACCIDENTS - I was in traction for 6 months/ we were both sick/we had some colds/I broke my leg/Mom was ill/

06. FAMILY CLOSER NOW - family a couple of miles awiay from home/ our family was pretty close by/the family was a lot closer than before/most of our family has moved closer to us/

07. LESS SOCIALIZING - we didn't go out as much/wasn't no one at home/I don't have as much demand socially/attend less social events/I didn't have anywhere to go/

08. WORKED/LESS TIME - wife worked/my job changed and I put in more hours/had to work on the holidays/I have a business and cannot leave it/I didn't have time this' year/

09. CAR PROBLEMS/NO CAR - I did not have my car the previous year/the way my car uses up gas/I had an accident and we were without a vehicle for a short while/car trouble/

10. TRAFFIC FEARS - too much hol iday traffic/accidents increase during the holiday season/so many people on the road who have been drinking/traffic in Florida was bad/

11. WEATHER CONDITIONS - we couldn't get out of town because of bad weather/I don't like to drive through the snow/ weather conditions/there was a blizzard/

12. TOOK A PLANE/TRAIN/BUS - we flew instead of drove to be with our family/I went on a plane/we rode the bus/went on a train/ 
Question $48-2$ continued

13. DIDN'T VISIT FAMILY/TOO FAR - we didn't go to see my family/didn't go visiting any relatives/didn't make the usual visit to my parents/didn't go to my brother's house/away from my family and relatives/daughters are farther away/

14. UNEMPLOYED - lack of employment/I was unemployed this year/ my husband was laid off/I quit work/

15. ELDERLY/RETIRED - too old to go anj'more/we're retired/it's due to my age/

16. CHANGED SHOPPING HABITS - I didn't take long shopping trips/ I shopped earlier/didn't drive around Christmas shopping as much/we have been shopping by mail/I don't shop with my" wife anymore/do less shopping/

17. OTHERS DROVE - someone else drove/traveled with son and his family/a friend driving instead of myself/traveled with other people/

18. DEATH IN FAMILY - my mother died/death in family/I was by myself due to my husband passing away/

19. OTHER

20. DON'T KNOW/NO ANSWER

49. Have you ever heard of Mothers Against

Drunk Drivers, or MADD?

Yes (TO 50-1 \& 50-2).......... Unsure (DO NOT READ) (TO 52) ...2

No $($ TO 52$) \ldots \ldots \ldots \ldots \ldots \ldots \ldots$

IF "YES" IN QUESTION 49, ASK:

50-I And can you tell me what MADD is or what they are trying to do?

01. PREVENT DRUNKEN DRIVING - trying to keep hard-core drinkers off the road/trying to get drunk drivers off the road P.T.A. is for them/prevent drunk drivers to keep them off the street/trying to stop drunk drivers from getting back on the road/trying to get drunks off the highway/

02. WANT STRONG LAWS - organization trying to strengthen drunk driving laws/to get legislation passed to get drunk drivers off the road/to get better laws against drunk driving/ their objective is to get more laws passed to be harder an drunk drivers/a group of mothers getting together trying to get stiffer legislation against drunk drivers/ 
Question 50-1 continued

03. WANT SEVERE PENALTIES - they are trying to get stricter penalties against drivers convicted of manslaughter due to drunk driving/they are trying to get stiffer fines and jail sentences for drunk drivers/the people involved are getting more severe punishment for drunk drivers/ they need stronger punishments/

04. PERSONALLY AFFECTED/ORUNK DRIVERS - an organization started by a woman whose son was killed by a drunk driver/they all have had a child killed in a car accident/a group started because children wiere killed by drunk drivers/parents of maimed children/mothers who have had their children killed by drunk drivers, and they try to console other victims/

05: REDUCE DRINKING-RELATED DEATHS/ACCIDENTS - prevent people driving when drunk and people getting killed/trying to keep drunk drivers off the road so that they will not kill the children/trying to decrease the number of highway deaths/trying to cut down on drunk driving deaths/ lower number of accidents/

06. STRICTER ENFORCEMENT - trying to enforce strict laws against D.W.I.Jstricter enforcement of drunk driving laws/trying to convict drunk drivers/

07. STOP DRINKERS FROM DRIVING - trying to keep people who drive off the roads/trying to put a stop to folks driving and drinking/they formed a group to keep people from drinking and driving/attempting to get the drinkers out from behind the wheel of a carl

08. AGAINST DRUNK DRIVERS - their efforts are against drunk drivers/control drunk drivers in this nation/getting the state to crack down on drunk drivers/women striking back at drunk drivers/against drunk drivers/complaining about drunk driving/angry at drunk drivers/

09. EDUCATE PUBLIC - to make people more aware of drunk drivers/ trying to develop a groundswell against drunk driving/ they are trying to bring the public some education/make drunk drivers more aware of the punishments they will receive/educate young people not to drink and drive/

10. JAIL SENTENCES FOR D.W.I. - they are trying to get mandatory jail sentences for drunk drivers/put drunk drivers in jail for 48 hours and if they have had an accident and killed...put them in prison/

11. SAW MOVIE/PUBLICITY - I saw a movie about them a few weeks ago/all I know is what I saw on T.V. about them/ seen a headline in the newspaper about that/I've just heard of them/ 
Question $50-1$ continued

12. RAISE DRINKING AGE/STOP TEEN DRUNKS - enforcing laws for teens not to drive during or after drinking/keep kids under 25 from drinking/get those kids off the road/ they are trying to have the drinking age raised/

13. DON'T KNOW/NO ANSWER

IF "YES" IN QUESTION 49, ASK:

50-2 Do you think Mothers Against Drunk Drivers is succeeding?

Yes (TO 51) ..................

Unsure (DO NOT READ) (TO 52)...2

IF "YES" IN QUESTION 50-2, ASK:

51. And in your own words, what are one or two reasons why you

feel that way?

01. NEW/STRICTER LAWS - passed new drunk drivitig laws/laws in

Colorado have gotten stricter partly due to this organization/just passed tougher laws here/we've had tougher drunk driving laws passed in the past year/

02. PUBLICITY - they get lots of publicity here/in advertising/ from hearing a lot about them on the news/hear more about it/they've gotten more publicity/they are getting more publicity than before/

03. PUBLIC AWARENESS - bringing awareness to the problem/ people are paying more attention to the problem/people are becoming more aware of the dangers of drunk drivers/ more people are aware of the problem/

04. STIFFER/INCREASED PENALTIES - in New York state there's a reaT push to get stiffer penalties/forcing the states to pass stiffer legislation against drunk drivers/drunk drivers are getting tougher sentences and more fines/ you get jailed for the first drunk driving offense now in Pennsylvania/

05. TELEVISION PRESENTATION - I happened to see their televised drama last week/I watched it on TV and I think they have expanded/I saw the movie last week/through the media/more known, especially on the medial

06. ENFORCING D.W.I. LAWS - police are really enforcing drunk driving laws/more arrests and convictions are being made/ in Seattle they are really cracking down on anyone caught drinking and driving/ 
Question 51 continued

07. WHAT I'VE SEEN/REA.D/HEARD - I'm hearing on news and in the paper/more publicity in newspapers/they're introducing their program to the media/from what I've read they've been successful/

08. RECOGNITION OF ORGANIZATION - recognition/they are succeeding because they are getting a lot of attention/getting more recognition now/

09. GROWING/EFFECTIVE - more public involvement/they are doing what they set out to do/more and more people becoming involved in this issue/they put on a good campaign/they've got more people joining now/.

10. FEWER DRUNKEN DRIVERS/ACCIDENTS - number of accidents due to alcohol has dropped/looking at the obituaries you can tell it's much less/less people on the road that are drunk/less accidents because of drunk drivers/getting many off the streets/

11. DOING A GOOO JOB - they are getting the message across/ it's very valuable, accomplish a lot/strong lobby and determination of them/people are paying more attention to the problems/it is a good cause that people are willing to support/

12. NEED STRONGER PUNISHMENT - it's better but the punishment isn't hard enough stillppeople are still getting away with drunk driving and killing people without a harsh punishment/some just get away scot-free/

13. NEED EDUCATION - "any" education in the public the better the chances are of getting results to the problem/good way to run them off the road - by educating others of the problem/educating lot of people/a lot of people who drink and drive are educated and through education they realize not to drive and drink/

14. NEED MORE TIME/SCOPE - it has helped but they need more time/they are not reaching enough to many judges and police/hasn't been a major change so far but their future looks hopeful/still have a long way to go/

15. NEEDLESS DEATHS/ACCIDENTS - it will stop deaths/seeing wrecks with young children involved/if they take someone's life they should be punished for it/they're just killing a lot of innocent people/

16. TRY HARDER/GET INVOLVED - feel that people should try harderlif they can do something about drunk drivers they should at least try/not enough people involved/
17. TAKING A STAND - people are speaking up for what's going
17. TAKING A STAND - people are speaking up for what's going by talking about it/


Question 51 continued

18. LEGISLATIVE ALLIES - they are complaining to the law about the drunk drivers/we have some bills in town and legislature - they will probably get passed/

19. DON'T KNOW/NO ANSWER

52. Which of the following best describes your use of safety belts compared to a year ago? (READ LIST)

1) I use them significantly more than I used to. $4 \%$

2) I use them slightly more than I used to. $15 \%$

3) I use them about the same. $75 \%$

4) I use them slightiy less than I used to. $2 \%$

5) I use them significantly less than I used to. $2 \%$

6) Don't know/no answer

IF "SIGNIFICANTLY MORE" OR "SLIGHTLY MORE" IN QUESTION 52, ASK:

53. And what are one or two reasons why you are usinil safety belts more than you used to?

01. TO BE SAFER - I believe they are safer/safety reasons, they work/because they save lives/to cut down on injuries/to protect myself more/I'm becoming brainwashed that they might be a little safer/it makes me feel more safe/trying to be more careful/they save lives/

02. FOR CHILDREN - have more children in the car since I drive them all to school/concern for my children/my children use them now/mostly for my son, to protect him/had a son, it really makes you more cautious in everything you do/I'm starting a family/children need to be in a safety harness always/

03. MORE CRAZY/DRUNK DRIVERS - I've noticed crazier people out there on the road/more people are speeding and drinking/ because of all the drunk drivers/don't trust other drivers/ there are more bad drivers on the road/

04. PERSONAL EXPERIENCE/WRECKS - I had to stop quickTy and my five year old lost several of her front teeth/I was in an accident and my seat belt saved me/I didn't have them in my truck when I wrecked it - I chipped my front teeth on the steering wheel/got a knee injury I don't think I would have if I had had on my seat belt/my wife had a serious accident/ 
Question 53 continued

05. GOOD/NEWER CAR DESIGN - I have a buzzer in my car which reminds me to fasten my seat belts/I have a new car and they are built right into it/my car will not start without the seat belts fastened/I have a new ca: and it's easier and more convenient/we got a new car with good seat belts/

06. PUBLICITY/FILMS - publicity/the advertisements/articles in paper/I saw on TV if you use seat belts it's safer/ seeing safety films at work/the campaigns going on here ads on TV/we are deluged with it/

07. MORE AWARE - more of an awareness of the importance of using them/I'm just more aware of the risks than I was a year ago/I think about it more/a little more awareness of the chance of being in an accident/I am more aware of the harm it does the older I get/

08. TRAFFIC PROBLEMS/ROAD CONDITIONS - because of the problem of traffic congestion/there are curves and hills, more hazardous conditions/the traffic problems are more severe/ the roads are in much worse condition than they ever have been/more people on the roads driving/there are more and more accidents happening everyday/

09. WITNESSED ACCIDENTS/SEEN VICTIMS - I've seen a lot cf accidents where people are getting thrown into the windshield/ I have witnessed a few accidents that people were hurt in/ I work in an ambulance and I've seen what happened/I see accidents all the time and they scare me/

10. FORMING HABIT - I'm trying to train myself to use it/getting used to them/trying to get into the habit of using safety belts/it's more of a habit with me now/

11. MORE DRIVING/LONGER TRIPS - I do more driving/because of the long distance trips I have to take to school/we have been taking more long trips/I'm driving more and taking longer trips/

12. NEW CAR/LESS SAFE CAR - I have a small car and I don't fee? as safe in it/I m now driving faster cars/when I got my new car I said I would use them and I do/the foreign cars don't have enough metal around them/a Jeep and it is known to be easily flipped/

13. ATTITUDE CHANGE - getting older, more sense/not in a rush getting into my vehicle/I'm a little more mature/more concerned about my $1 \mathrm{ife} / \mathrm{I}^{\prime}$ ve just calmed down/

14. SET EXAMPLE/TEACH CHILOREN - I'm trying to set a good example for my children/so children will use them/to set an example for the kids/my son took drivers ed course and I'm helping teach him how to drive/I have two little ones to set an example for/ 
Question 53 continued

15. CHILDREN'S CAR SEATS - because of the grandchildren - even

if I just go a block, I have one question - will I get a

ticket if I don't have my grandchildren in a car seat/I

put my baby into a car seat now and so I just go ahead

and put myself in a seat belt too/

16. LAST YEAR SURVEY - after I did this survey a year ago, it made me more aware of the fact I should try to wear them more/

17. OTHER

IF "SLIGHTLY LESS" OR "SIGNIFICANTLY LESS" IN QUESTION 52, ASK:

54. And what are one or two reasons why you are using safety belts

less than you used to?

01. UNCOMFORTABLE - I am a short person and the seat belt comes across my neck and it's very uncomfortable/they are very uncomfortable/I think we should be able to fit the seat belts to everyone's different sicied bodies/I don't think I can move as well in them/pain in the neck, uncomfortable/

02. JUST HABIT/BROKE HABIT - I broke that habit and I never remember to wear them/I fell out of the habit and I just never remember/just got into the habit of not buckling up/

03. NOT SAFE - my life was saved when I didn't wear one/they couTdn't get the lady out of the car, they had to saw it apart/I have a friend who died in an automobile accident because he couldn't get his seat belts off/my last auto accident, I had my belt on then and had a hell of a time getting it off/

04. IN A HURRY/DON'T TAKE TIME - always in a hurry, don't take time/I never take the time to think about it/I' $m$ always in a hurry to get in and out of my carl

05. NOT EFFECTIVE/NO NEED - I don't believe they help you any! I didn't feel I needed them/

06. DRIVE LESS - don't drive as much as I used to/driving less since I fell and I'm also older/

07. SPECIFIC MENTIONS - because I'm pregnant/I only wear seat beits in bad weather/only use when have younger children in carl

08. DON'T KNOW/NO ANSWER 
55. Do you have any children 5 years and under in your household?

Yes (T0 56)................

No $($ TO 57$) \ldots \ldots \ldots \ldots \ldots \ldots \ldots 2$

IF "YES" IN QUESTION 55, ASK:

56. How often are children 5 and under in your household fastened into

infant carriers or child safety seats

-- all the time, most of the time, sometimes, or almost never?

All the time...............

Most of the time.............

Sometimes.................

Almost never............4

57. What do you believe is the most frequent cause of death among persons under age 35 ? Is it... (READ ALTERNATIVES)

Heart attack..............

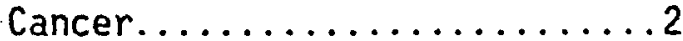

Other disease...............

Alcohol-related traffic

accidents..............4

Other traffic accidents......5

Don't know/no answer.........6

58. How many cigarettes do you smoke a day -- (READ ALTERNATIVES)

Don't smoke cigarettes........1

Less than $1 / 2$ pack...........

$1 / 2$ to less than 1 pack.......

1 pack or more...........

59. How often do you visit the dentist to have your teeth checked? (READ ALTERNATIVES)

Every 6 months.............

Every year................

Every 2 years or more.........3 
60. Do you jog, cycle, swim or exercise regularly at least twice a week?

Yes.....................1

No.

61. Within the past three years, have you undertaken any other activities or taken other actions on your own to maintain or improve your health?

Yes.....................

$(42 \%)$

No.....................

Now, just a few final questions for statistical purposes only --

62. What is your age, please?

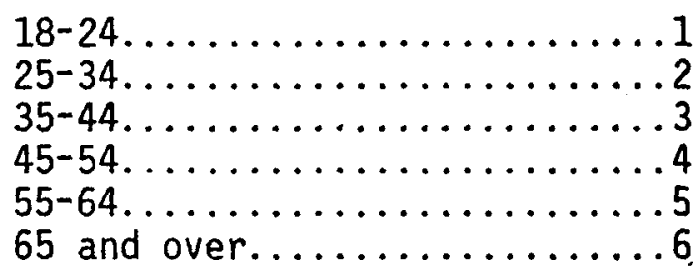

63. What is the last grade of school you completed? (READ LIST)

Less than high school......... I

High school graduate...........2

64. Of the following types of work, which one describes what the head of the household does?

(READ LIST)
Self-employed..............

White-collar...............

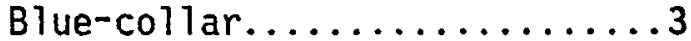

Government worker..........4

Farmer/rancher............5

Not in the labor force

(student, housewife, retired)............. 6

Unemployed.......................

other (SPECIFY)
(13\%) 
65. Which of the following best describes where you live? (READ ALTERNATIVES)

In a big city.............

$(22 \%)$

In the suburbs around a big city.................

In a smill city.............

In a town or village.........4

In the country............. 5

66. SeX (BY OBSERVATION)

Male..................

Female.................

$(50 \%)$ 